\title{
Anyons in an exactly solved model and beyond
}

\author{
Alexei Kitaev \\ California Institute of Technology, Pasadena, CA 91125, U.S.A. \\ e-mail: kitaev@iqi.caltech.edu
}

August 14, 2005

\begin{abstract}
A spin $1 / 2$ system on a honeycomb lattice is studied. The interactions between nearest neighbors are of XX, YY or ZZ type, depending on the direction of the link; different types of interactions may differ in strength. The model is solved exactly by a reduction to free fermions in a static $\mathbb{Z}_{2}$ gauge field. A phase diagram in the parameter space is obtained. One of the phases has an energy gap and carries excitations that are Abelian anyons. The other phase is gapless, but acquires a gap in the presence of magnetic field. In the latter case excitations are non-Abelian anyons whose braiding rules coincide with those of conformal blocks for the Ising model. We also consider a general theory of free fermions with a gapped spectrum, which is characterized by a spectral Chern number $\nu$. The Abelian and non-Abelian phases of the original model correspond to $\nu=0$ and $\nu= \pm 1$, respectively. The anyonic properties of excitation depend on $\nu$ mod 16 , whereas $\nu$ itself governs edge thermal transport. The paper also provides mathematical background on anyons as well as an elementary theory of Chern number for quasidiagonal matrices.
\end{abstract}

\section{Contents}

Introduction

$\begin{array}{lll}1 \text { The model } & 10\end{array}$

$\begin{array}{lll}2 & \text { Representing spins by Majorana operators } & 11\end{array}$

2.1 A general spin-fermion transformation . . . . . . . . . . . . . . . . . . 11

2.2 Application to the concrete model . . . . . . . . . . . . . . . . 12

2.3 Path and loop operators . . . . . . . . . . . . . . . . . . . . . 14

$\begin{array}{llr}3 & \text { Quadratic Hamiltonians } & 14\end{array}$

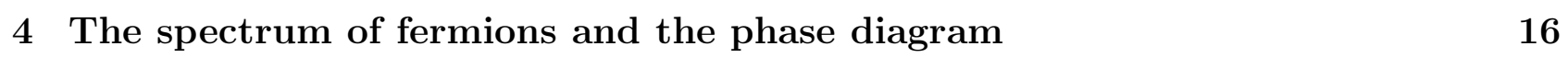

$\begin{array}{ll}5 \text { Properties of the gapped phases } & 19\end{array}$

5.1 Perturbation theorv studv . . . . . . . . . . . . . . . . . . . . . . . . . . . 19

5.2 Abelian anvons . . . . . . . . . . . . . . . . . . . . 21 
6 Phase $B$ acquires a gap in the presence of magnetic field 23

6.1 The conic singularitv and the time-reversal svmmetry . . . . . . . . . . . 23

6.2 Derivation of an effective Hamiltonian . . . . . . . . . . . . . . . . . 24

6.3 The spectrum and the Chern number . . . . . . . . . . . . . . . . 25

\begin{tabular}{lll}
\hline 7 & Edge modes and thermal transport & 26
\end{tabular}

8 Non-Abelian anvons 28

8.1 Bulk-edge correspondence . . . . . . . . . . . . . . . . . . . . . . . . . . . . . . . . 29

8.2 Unpaired Maiorana modes . . . . . . . . . . . . . . . . . . . . . . . . . . 31

8.3 Fusion and braiding rules . . . . . . . . . . . . . . . . . . . . . . . . . . 32

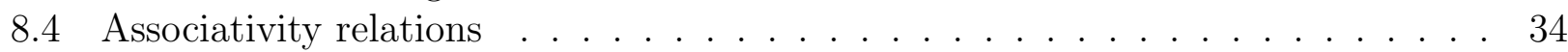

8.5 Algebraic consistency . . . . . . . . . . . . . . . . . . . . . . . . 37

8.6 Final details . . . . . . . . . . . . . . . . . . . . . . . . . . . . . 40

9 The sixteen-fold wav 42

10 Odds and ends 43

A Numerical results on the stability of the vortex-free phase 45

\begin{tabular}{ll}
\hline B Edge modes in phases $B_{4}$ & 47
\end{tabular}

\begin{tabular}{ll}
\hline C Quasidiagonal matrices & 49
\end{tabular}

C.1 The flow of a unitary matrix . . . . . . . . . . . . . . . . . 51

C.2 General setting . . . . . . . . . . . . . . . . . . . . . . . . 53

C.3 Chern number . . . . . . . . . . . . . . . . . . . . . . . . . . 54

C.4 Majorana numbers . . . . . . . . . . . . . . . . . . . . . . . 57

D Some remarks on the chiral central charge $\quad 59$

D.1 The edge energy current and a bulk 2-current . . . . . . . . . . . . . . . . 60

D.2 Modular transformations bevond CFT . . . . . . . . . . . . . . . . 63

\begin{tabular}{ll}
\hline E Algebraic theory of anyons & 65
\end{tabular}

E.1 Fusion theorv . . . . . . . . . . . . . . . . . . . . . . . . 67

E.2 Particle-antiparticle duality . . . . . . . . . . . . . . . . . . . . . . 74

E.3 Braiding and topological spin . . . . . . . . . . . . . . . . . 80

E.4 Verlinde algebra and topological $S$-matrix $\ldots \ldots \ldots \ldots$. . . . . . . . 84

E.5 Braiding nondegeneracv $=$ modularity $\ldots \ldots \ldots \ldots$. . . . . . 85

E.6 Gauge freedom and Ocneanu rigidity . . . . . . . . . . . . . . . . . . . . . 89

E.7 Categorical formalism (aside) . . . . . . . . . . . . . . . . . . . . 94

\begin{tabular}{ll}
\hline F Weak svmmetry breaking & 104
\end{tabular}

\begin{tabular}{ll}
\hline Acknowledoments & 106
\end{tabular}

\begin{tabular}{ll}
\hline References & 107
\end{tabular} 


\section{Comments to the contents: What is this paper about?}

Certainly, the main result of the paper is an exact solution of a particular two-dimensional quantum model. However, I was sitting on that result for too long, trying to perfect it, derive some properties of the model, and put them into a more general framework. Thus many ramifications have come along. Some of them stem from the desire to avoid the use of conformal field theory, which is more relevant to edge excitations rather than the bulk physics. This program has been partially successful, but some rudiments of conformal field theory (namely, the topological spin $\theta_{a}=e^{2 \pi i\left(h_{a}-\bar{h}_{a}\right)}$ and the chiral central charge $\left.c_{-}=c-\bar{c}\right)$ are still used.

The paper is self-contained and provides an introduction into the subject. For most readers, a good strategy is to follow the exposition through the beginning of Sec. 8. Nonabelian anyons and take a glance at the rest of that section, where things become more technical. But the mathematically inclined reader may be interested in those details, as well as some of the appendices. I have tried to make the paper modular so that some parts of it can be understood without detailed reading of the other. This has caused some redundancy though.

Appendix E. Algebraic theory of anyons is an elementary introduction into unitary modular categories, which generalizes the discussion in Sec. 8 .

Appendix C. Quasidiagonal matrices is also mostly expository but some of the arguments may be new. It begins with a simplified treatment of "operator flow" and "noncommutative Chern number" (the latter has been used to prove the quantization of Hall conductivity in disordered systems [1]), but the main goal is to explain "unpaired Majorana modes", a certain parity phenomenon related to the Chern number.

Appendix $D$ on the chiral central charge and Appendix $\mathbb{E}$ on weak symmetry breaking contain some raw ideas that might eventually develop into interesting theories.

\section{Introduction}

Overview of the subject. Anyons are particles with unusual statistics (neither Bose nor Fermi), which can only occur in two dimensions. Quantum statistics may be understood as a special kind of interaction: when two particles interchange along some specified trajectories, the overall quantum state is multiplied by $e^{i \varphi}$. In three dimensions, there is only one topologically distinct way to swap two particles. Two swaps are equivalent to the identity transformation, hence $e^{i \varphi}= \pm 1$. On the contrary, in two dimensions the double swap corresponds to one particle making a full turn around the other; this process is topologically nontrivial. Therefore the exchange phase $\varphi$ can, in principle, have any value - hence the name "anyon". (However, a stability consideration requires that $\varphi$ be a rational multiple of $2 \pi$.) Of course, the real question is whether such particles exist in nature or can be built somehow, but we will follow the historic path, approaching the problem from the mathematical end.

The study of anyons was initiated by Wilczek [2, 3] in early 1980's. He proposed a simple but rather abstract model, which was based on $(2+1)$-dimensional electrodynamics. This theory has integer electric charges and vortices carrying magnetic flux (which is a real number defined up to an integer). Considered separately, both kinds of particles are bosons. But when a charge $q$ goes around a vortex $v$, it picks up the phase $2 \pi q v$, known as the Aharonov-Bohm phase. Thus, charges and vortices have nontrivial mutual statistics and therefore must be called 
anyons when considered together. Moreover, composite objects $(q, v)$ consisting of a charge and a vortex are anyons by themselves because they have nontrivial exchange phase $\varphi_{(q, v)}=2 \pi q v$.

A general way to describe quantum statistics is to consider particle worldlines in the $(2+1)$ dimensional space-time. Such worldlines form a braid, therefore the statistics is characterized by a representation of the braid group. In the preceding discussion we assumed that braiding is characterized just by phase factors, i.e., that the representation is one-dimensional. The corresponding anyons are called Abelian. But one can also consider multidimensional representations of the braid group; in this case the anyons are called non-Abelian. Actually, it may not be so important how the braid group acts, but the very existence of a multidimensional space associated with several particles is a key feature. Vectors in this space are quantum states that have almost the same energy (see discussion of topological quantum computation below).

Historically, the theory of non-Abelian anyons emerged from conformal theory (CFT). However, only topological and algebraic structure in CFT is relevant to anyons. Different pieces of this structure were discovered in a colossal work of many people, culminating in the paper by Moore and Seiberg [4. Witten's work on quantum Chern-Simons theory [5] was also very influential. A more abstract approach (based on local field theory) was developed by Fredenhagen, Rehren, and Schroer [6] and by Frohlich and Gabbiani [7].

The most amazing thing about anyons is that they actually exist as excitations in some condensed matter systems. Such systems also have highly nontrivial ground states that are described as possessing topological order. The best studied example (both theoretically and experimentally) is the Laughlin state [8] in the fractional quantum Hall system at the filling factor $\nu=1 / 3$. It carries Abelian anyons with exchange phase $\varphi=\pi / 3$ and electric charge $\pm 1 / 3$. It is the fractional value of the charge that was predicted in original Laughlin's paper and confirmed by several methods, in particular by a shot noise measurement [9, 10]. The statistical phase is a subtler property which is deduced theoretically [11, 12]; a nontrivial experimental test has been performed recently using quasiparticle tunneling [13].

A different kind of state is observed at the filling factor $\nu=5 / 2$, though it is more fragile and less studied experimentally. There is much evidence suggesting that this system is described by a beautiful theory proposed by Moore and Read [14, 15. The Moore-Read state admits nonAbelian anyons with charge $\pm 1 / 4$. If $2 n$ such particles are present, the associated Hilbert space has dimension $2^{n-1}$. (The non-Abelian anyons studied in this paper have similar properties, though there is no electric charge.)

The notion of anyons assumes that the underlying state has an energy gap (at least for topologically nontrivial quasiparticles). Otherwise excitations are not localizable and braiding may not be defined. Note that if all excitations are gapped, then all equal-time correlators decay exponentially with distance [16].

An example of anyons in a spin-1/2 system originates from the theory of resonating valence bond (RVB). The idea of RVB was put forward by Anderson [17] and used later as a model of the undoped insulating phase in high- $T_{c}$ cuprate superconductors [18. Without electrically charged holes, the problem seems to be described adequately by a Heisenberg-like Hamiltonian, but its solution has proved very difficult. Several variants of an RVB state have been proposed, both gapless and gapped. Here we discuss a particular gapped RVB phase, namely the one which is realized on the triangular lattice [19, but which apparently exists on the square lattice as well. This phase admits quasiparticles of four types: trivial excitations (such as spin waves), spinons (spin-1/2 fermions, which are conserved modulo 2 ), $\mathbb{Z}_{2}$-vortices (spinless bosons, also 


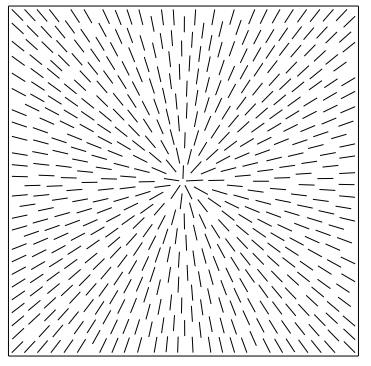

a)

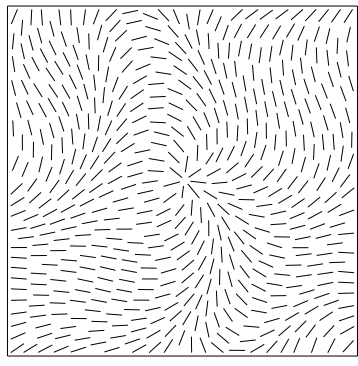

b)

Figure 1: A classical vortex (a) distorted by fluctuations (b).

called visons), and spinon-vison composites [20]. The mutual statistics of spinons and visons is characterized by the Aharonov-Bohm factor -1 , therefore the composite particles are bosons. Note that the relevance of this theory to cuprate superconductors is under debate. Senthil and Fisher proposed an interesting way to detect visons in these materials 21, but the experiment gave a negative result [22]. However, some kind of RVB state is likely to realize in a different material, $\mathrm{Cs}_{2} \mathrm{CuCl}_{4}$. This conclusion is drawn from neutron-scattering experiments that have shown the presence of spin-1/2 excitations [23].

Anyonic particles are best viewed as a kind of topological defects that reveal non-trivial properties of the ground state. Thus anyons carry some topological quantum numbers which make them stable: a single particle cannot be annihilated locally but only through the fusion with an antiparticle. An intuitive way to picture an anyon is to imagine a vortex in a medium with a local order parameter (see Fig. 17). Now suppose that quantum fluctuations are so strong that the order parameter is completely washed out and only the topology remains (see Fig. 1b). Of course, that is only a rough illustration. It resembles the Kosterlitz-Thouless phase with power-law correlation decay, while in anyonic systems correlations decay exponentially due to the energy gap.

A real example can be constructed with spins on the edges of a square lattice. Basis states of the spins are described by the variables $s_{j}= \pm 1$, which may be regarded as a $\mathbb{Z}_{2}$ gauge field (i.e., "vector potential"), whereas the "magnetic field intensity" on plaquette $p$ is given by

$$
w_{p}=\prod_{j \in \operatorname{boundary}(p)} s_{j}
$$

We say that there is a vortex on plaquette $p$ if $w_{p}=-1$. Now we may define the vortex-free state:

$$
|\Psi\rangle=c \sum_{\substack{\mathbf{s}: \\ w_{p}(\mathbf{s})=1 \\ \text { for all } p}}|\mathbf{s}\rangle, \quad \text { where } \mathbf{s}=\left(s_{1}, \ldots, s_{N}\right)
$$

( $c$ is a normalization factor). The state with a single vortex on a given plaquette is defined similarly. It is clear that the vortex can be detected by measuring the observable $\prod_{j \in l} \sigma_{j}^{z}$ for any enclosing path $l$, though no local order parameter exists.

The state (2) can be represented as the ground state of the following Hamiltonian with 
four-body interaction [24]:

$$
H=-J_{e} \sum_{\text {vertices }} A_{s}-J_{m} \sum_{\text {plaquettes }} B_{p}, \quad \text { where } \quad A_{s}=\prod_{\operatorname{star}(s)} \sigma_{j}^{x}, \quad B_{p}=\prod_{\text {boundary }(p)} \sigma_{j}^{z} \text {. }
$$

Its elementary excitations are $\mathbb{Z}_{2}$-charges with energy $2 J_{e}$ and vortices with energy $2 J_{m}$. Certain essential features of this model are stable to small local perturbations (such as external magnetic field or Heisenberg interaction between neighboring spins). Note that the robust characteristic of excitations is not the energy or the property of being elementary, but rather superselection sector. It is defined as a class of states that can be transformed one to another by local operators. This particular model has the vacuum sector 1 , the charge sector $e$, the vortex sector $m$, and a charge-vortex composite $\varepsilon$. Particles of type $e$ and $m$ are bosons with nontrivial mutual statistics, whereas $\varepsilon$ is a fermion. Thus, the model represents a universality class of topological order - actually, the same class as RVB.

Anyonic superselection sectors may or may not be linked to conventional quantum numbers, like spin or electric charge. Most studies have been focused on the case where anyons carry fractional electric charge or half-integer spin. Such anyons are potentially easier to find experimentally because they contribute to collective effects (in particular, electric current) or have characteristic selection rules for spin-dependent scattering. Chargeless and spinless quasiparticles are generally harder to identify. But anyons, by virtue of their topological stability, must have some observable signatures. For example, anyons can be trapped by impurities and stay there for sufficiently long time, modifying the spectrum of local modes (magnons, excitons, etc.). However, effective methods to observe anyons are yet to be found.

Thus, the hunt for anyons and topological order is a difficult endeavor. Why do we care? First, because these are conceptually important phenomena, breaking some paradigms. In particular, consider these principles (which work well and provide important guidance in many cases):

1. Conservation laws come from symmetries (by Noether's theorem or its quantum analogue);

2. Symmetries are initially present in the Hamiltonian (or Lagrangian), but may be spontaneously broken.

Let us limit our discussion to the case of gauge symmetries and local conservation laws, which are described by fusion rules between superselection sectors. A profound understanding of the first principle and its underlying assumptions is due to Doplicher and Roberts [25, 26. They proved that any consistent system of fusion rules for bosons is equivalent to the multiplication rules for irreducible representations of some compact group. Fermions also fit into this framework. However, anyonic fusion rules are not generally described by a group! As far as the second principle is concerned, topological order does not require any preexisting symmetry but leads to new conservation laws. Thus, the formation of topological order is exactly the opposite of symmetry breaking!

Topological quantum computation. A more practical reason to look for anyons is their potential use in quantum computing. In Ref. 24] I suggested that topologically ordered states can serve as a physical analogue of error-correcting quantum codes. Thus, anyonic systems 
provide a realization of quantum memory that is protected from decoherence. Some quantum gates can be implemented by braiding; this implementation is exact and does not require explicit error correction. Freedman, Larsen, and Wang [27] proved that for certain types of non-Abelian anyons braiding enables one to perform universal quantum computation. This scheme is usually referred to as topological quantum computation (TQC).

Let us outline some basic principles of TQC. First, topologically ordered systems have degenerate ground states under certain circumstances. In particular, the existence of Abelian anyons implies the ground state degeneracy on the torus [28. Indeed, consider a process in which a particle-antiparticle pair is created, one of the particles winds around the torus, and the pair is annihilated. Such a process corresponds to an operator acting on the ground state. If $A$ and $B$ are such operators corresponding to two basic loops on the torus, then $A B A^{-1} B^{-1}$ describes a process in which none of the particles effectively crosses the torus, but one of them winds around the other. If the Aharonov-Bohm phase is nontrivial, then $A$ and $B$ do not commute. Therefore they act on a multidimensional space.

Actually, the degeneracy is not absolute by very precise. It is lifted due to virtual particle tunneling across the torus, but this process is exponentially suppressed. Therefore the distance between ground energy levels is proportional to $\exp (-L / \xi)$, where $L$ the linear size of the torus and $\xi$ is some characteristic length, which is related to the gap in the excitation spectrum.

In non-Abelian systems, degeneracy occurs even in the planar geometry when several anyons are localized in some places far apart from each other (it is this space of quantum states the braid group acts on). The underlying elementary property may be described as follows: two given non-Abelian particles can fuse in several ways (like multi-dimensional representations of a non-Abelian group). For example, the non-Abelian phase studied in this paper has the following fusion rules:

$$
\varepsilon \times \varepsilon=1, \quad \varepsilon \times \sigma=\sigma, \quad \sigma \times \sigma=1+\varepsilon,
$$

where 1 is the vacuum sector, and $\varepsilon$ and $\sigma$ are some other superselection sectors. The last rule is especially interesting: it means that two $\sigma$-particles may either annihilate or fuse into an $\varepsilon$-particle. But when the $\sigma$-particles stay apart, 1 and $\varepsilon$ correspond to two quantum states, $\left|\psi_{1}^{\sigma \sigma}\right\rangle$ and $\left|\psi_{\varepsilon}^{\sigma \sigma}\right\rangle$. These states are persistent. For example, if we create $\left|\psi_{\varepsilon}^{\sigma \sigma}\right\rangle$ by splitting an $\varepsilon$ into two $\sigma$ 's, wait some time, and fuse the $\sigma$-particles back, we will still get an $\varepsilon$-particle.

Here is a subtler property: the fusion states $\left|\psi_{1}^{\sigma \sigma}\right\rangle$ and $\left|\psi_{\varepsilon}^{\sigma \sigma}\right\rangle$ are practically indistinguishable and have almost the same energy. In fact, a natural process that "distinguishes" them by multiplying by different factors is tunneling of a virtual $\varepsilon$-particle between the fixed $\sigma$-particles (which is possible since $\sigma \times \varepsilon=\sigma$ ). However, $\varepsilon$-particles are gapped, therefore this process is exponentially suppressed. Of course, this explanation depends on many details, but it is a general principle that different fusion states can only be distinguished by transporting a quasiparticle. Such processes are unlikely even in the presence of thermal bath and external noise, as long as the temperature and the noise frequency are much smaller than the gap.

In the above example, the two-particle fusion states $\left|\psi_{1}^{\sigma \sigma}\right\rangle$ and $\left|\psi_{\varepsilon}^{\sigma \sigma}\right\rangle$ cannot form coherent superpositions because they belong to different superselection sectors ( 1 and $\varepsilon$, resp.). In order to implement a qubit, one needs four $\sigma$-particles. A logical $|0\rangle$ is represented by the quantum state $\left|\xi_{1}\right\rangle$ that is obtained by creating the pairs $(1,2)$ and $(3,4)$ from the vacuum (see Fig. 2). A logical $|1\rangle$ is encoded by the complementary state $\left|\xi_{\varepsilon}\right\rangle$ : we first create a pair of $\varepsilon$-particles, and then split each of them into a $\sigma \sigma$-pair. Note that both states belong to the vacuum sector and 


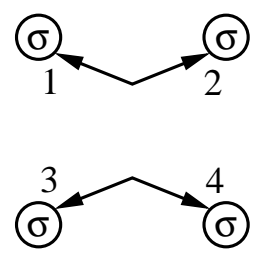

$\left|\xi_{1}\right\rangle$

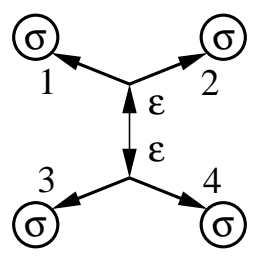

$\left|\xi_{\varepsilon}\right\rangle$

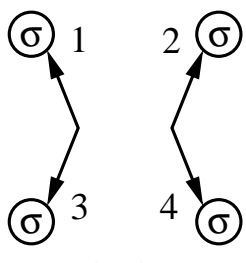

$\left|\eta_{1}\right\rangle$

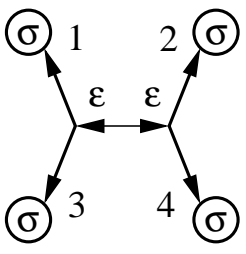

$\left|\eta_{\varepsilon}\right\rangle$

Figure 2: Four ways to initialize an anyonic qubit.

therefore can form superpositions. Also shown in Fig. 2 are two alternative ways to initialize the qubit, $\left|\eta_{1}\right\rangle$ and $\left|\eta_{\varepsilon}\right\rangle$. The detailed analysis presented in Secs. 8.4 and 8.5 implies that

$$
\left|\eta_{1}\right\rangle=\frac{1}{\sqrt{2}}\left(\left|\xi_{1}\right\rangle+\left|\xi_{\varepsilon}\right\rangle\right), \quad\left|\eta_{\varepsilon}\right\rangle=\frac{1}{\sqrt{2}}\left(\left|\xi_{1}\right\rangle-\left|\xi_{\varepsilon}\right\rangle\right) .
$$

Therefore we can perform the following gedanken experiment. We create the state $\left|\xi_{1}\right\rangle$ and then measure the qubit in the $\left\{\left|\eta_{1}\right\rangle,\left|\eta_{\varepsilon}\right\rangle\right\}$-basis by fusing the pairs $(1,3)$ and $(2,4)$. With probability $1 / 2$ both pairs annihilate, and with probability $1 / 2$ we get two $\varepsilon$-particles. One can also think of a simple robustness test for quantum states: if there is no decoherence, then both $\left|\xi_{1}\right\rangle$ and $\left|\eta_{1}\right\rangle$ are persistent.

As already mentioned, braiding is described by operators that are exact (up to virtual quasiparticle tunneling). Indeed, the operators of counterclockwise exchange between two particles ( $R$-matrices) are related to the fusion rules by so-called hexagon equations and pentagon equation. We will see on concrete exapmles that these equations have only a finite number of solutions and therefore do not admit small deformations. In general, it is a nontrivial theorem known as Ocneanu rigidity [29, 30], see Sec. E.6.

Thus, we have all essential elements of a quantum computer implemented in a robust fashion: an initial state is made by creating pairs and/or by splitting particles, unitary gates are realized by braiding, and measurements are performed by fusion. This "purely topological" scheme is universal for sufficiently complicated phases such as the $k=3$ parafermion state [31, lattice models based on some finite groups (e.g. $S_{5}$ [24], $A_{5}$ [32, 33] and $S_{3}$ [34]), and double ChernSimons models 35, 36, 37. Unfortunately, the model studied in this paper is not universal in this sense. One can, however, combine a topologically protected quantum memory with a nontopological realization of gates (using explicit error correction). Note that some weak form of topological protection is possible even in one-dimensional Josephson junction arrays [38, which is due to the build-in U(1)-symmetry. Several other schemes of Josephson junctionbased topological quantum memory have been proposed recently [39, 40, 41].

Unlike many other quantum computation proposals, TQC should not have serious scalability issues. What is usually considered an initial step, i.e., implementing a single gate, may actually be close to the solution of the whole problem. It is an extremely challenging task, though. It demands the ability to control individual quasiparticles, which is beyond the reach of present technology. One should however keep in mind that the ultimate goal is to build a practical quantum computer, which will contain at least a few hundred logical qubits and involve errorcorrecting coding: either in software (with considerable overhead) or by topological protection or maybe by some other means. At any rate, that is a task for the technology of the future. 
But for the meantime, finding and studying topological phases seems to be a very reasonable goal, also attractive from the fundamental science perspective.

Comparison with earlier work and a summary of the results. In this paper we study a particular exactly solvable spin model on a two-dimensional lattice. It only involves two-body interactions and therefore is simpler than Hamiltonian (3) considered in [24, but the solution is less trivial. It is not clear how to realize this model in solid state, but an optical lattice implementation has been proposed 42 .

The model has two phases (denoted by $A$ and $B$ ) which occur at different values of parameters. The exact solution is obtained by a reduction to free real fermions. Thus quasiparticles in the system may be characterized as fermions and $\mathbb{Z}_{2}$-vortices. Vortices and fermions interact by the Aharonov-Bohm factor equal to -1 . In phase $A$ the fermions have an energy gap, and the vortices are bosons that fall into two distinct superselection sectors. (Interestingly enough, the two types of vortices have identical physical properties and are related to each other by a lattice translation.) The overall particle classification, fusion rules, and statistics are the same as in the model (2) or RVB. In phase $B$ the fermions are gapless and there is only one type of vortices with undefined statistics. Adding a magnetic field to the Hamiltonian opens a gap in the fermionic spectrum, and the vortices become non-Abelian anyons. The difference between the vortex statistics in phase $A$ and phase $B$ with the magnetic field may be attributed to different topology of fermionic pairing.

Topological properties of Fermi-systems were first studied in the theory of integer quantum Hall effect [43, 44]. Let us outline the main result. To begin with, the Hall conductivity of noninteracting electrons in a periodic potential (e.g., in the Hofstadter model with $m / n$ flux quanta per plaquette) is expressed in terms of a single-electron Hamiltonian in the Fourier basis. Such a Hamiltonian is an $n \times n$ matrix that depends on the momentum $\mathbf{q}$. For each value of $q$ one can define a subspace $\mathcal{L}(\mathbf{q}) \subseteq \mathbb{C}^{n}$ that is associated with negative-energy states, i.e., ones that are occupied by electrons. Thus, a vector bundle over the momentum space is defined. The quantized Hall conductivity is proportional to the Chern number of this bundle. Bellissard at al [1] have generalized this theory to disordered systems by using a powerful mathematical theory called noncommutative geometry [45].

Even more interesting topological phenomena occur when the number of particles is not conserved (due to the presence of terms like $a_{j}^{\dagger} a_{k}^{\dagger}$, as in the mean-field description of superconductors). In this case the single-electron Hamiltonian is replaced by a more general object, the Bogolyubov-Nambu matrix. It also has an associated Chern number $\nu$, which is twice the number defined above when the previous definition is applicable. But in general $\nu$ is an arbitrary integer. The first physical example of this kind, the ${ }^{3} \mathrm{He}-\mathrm{A}$ film, was studied by Volovik [46]. Volovik and Yakovenko [4] showed that the Chern number in this system determines the statistics of solitons. More recently, Read and Green [4] considered BCS pairing of spinless particles with angular momentum $l=-1$. They identified a "strong pairing phase" with zero Chern number and a "weak pairing phase" with $\nu=1$. The latter is closely related to the Moore-Read state and has non-Abelian vortices and chiral edge modes.

In the present paper, these results are generalized to an arbitrary Fermi-system described by a quadratic Hamiltonian on a two-dimensional lattice. We show that $\mathbb{Z}_{2}$-vortices are Abelian particles when the Chern number $\nu$ is even and non-Abelian anyons when $\nu$ is odd. The nonAbelian statistics is due to unpaired Majorana modes associated with vortices. Our method 
relies on a quasidiagonal matrix formalism (see Appendix C), which is similar to, but more elementary than, noncommutative geometry. It can also be applied to disordered systems.

Furthermore, we find that there are actually 16 (8 Abelian and 8 non-Abelian) types of vortex-fermion statistics, which correspond to different values of $\nu$ mod 16 . Only three of them (for $\nu=0, \pm 1$ ) are realized in the original spin model. We give a complete algebraic description of all 16 cases, see tables on pages 30, 41, and 42 .

\section{The model}

We study a spin-1/2 system in which spins are located at the vertices of a honeycomb lattice, see Fig. 3a. This lattice consists of two equivalent simple sublattices, referred to as "even" and

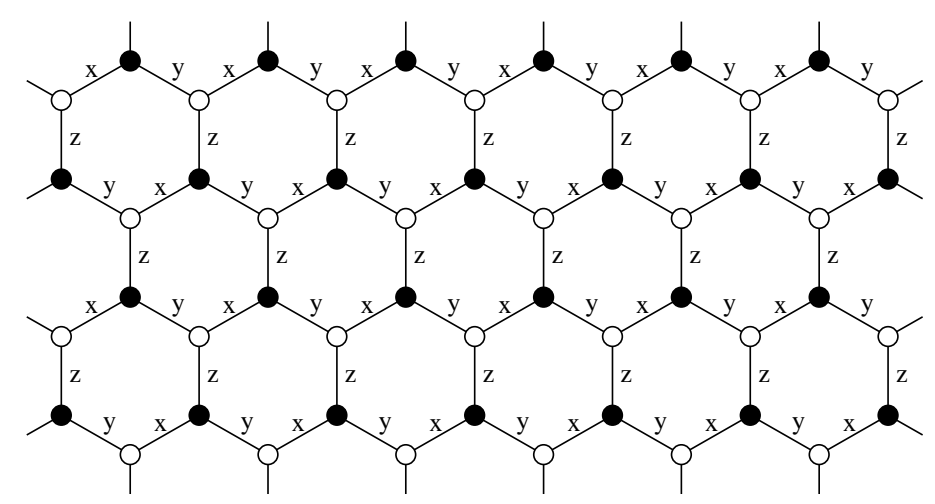

a)

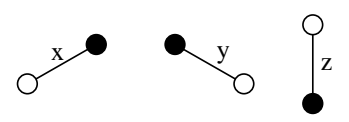

b)

Figure 3: Three types of links in the honeycomb lattice.

"odd" (they are shown by empty and full circles in the figure). A unit cell of the lattice contains one vertex of each kind. Links are divided into three types, depending on their direction (see Fig. 3b); we call them " $x$-links", " $y$-links", and " $z$-links". The Hamiltonian is as follows:

$$
H=-J_{x} \sum_{x \text {-links }} \sigma_{j}^{x} \sigma_{k}^{x}-J_{y} \sum_{y \text {-links }} \sigma_{j}^{y} \sigma_{k}^{y}-J_{z} \sum_{z \text {-links }} \sigma_{j}^{z} \sigma_{k}^{z}
$$

where $J_{x}, J_{y}, J_{z}$ are model parameters.

Let us introduce a special notation for the individual terms in the Hamiltonian:

$$
K_{j k}= \begin{cases}\sigma_{j}^{x} \sigma_{k}^{x}, & \text { if }(j, k) \text { is an } x \text {-link; } \\ \sigma_{j}^{x} \sigma_{k}^{y}, & \text { if }(j, k) \text { is an } y \text {-link; } \\ \sigma_{j}^{x} \sigma_{k}^{z}, & \text { if }(j, k) \text { is an } z \text {-link. }\end{cases}
$$

Remarkably, all operators $K_{j k}$ commute with the following operators $W_{p}$, which are associated to lattice plaquettes (i.e., hexagons):

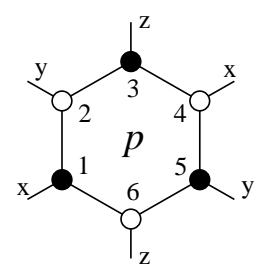

$$
W_{p}=\sigma_{1}^{x} \sigma_{2}^{y} \sigma_{3}^{z} \sigma_{4}^{x} \sigma_{5}^{y} \sigma_{6}^{z}=K_{12} K_{23} K_{34} K_{45} K_{56} K_{61}
$$


Here $p$ is a label of the plaquette. Note that different operators $W_{p}$ commute with each other.

Thus Hamiltonian (4) has the set of "integrals of motion" $W_{p}$, which greatly simplifies the problem. In order to find eigenstates of the Hamiltonian, we first divide the total Hilbert space $\mathcal{L}$ into sectors - eigenspaces of $W_{p}$, which are also invariant subspaces of $H$. This can be written as follows:

$$
\mathcal{L}=\bigoplus_{w_{1}, \ldots, w_{m}} \mathcal{L}_{w_{1}, \ldots, w_{m}}
$$

where $m$ is the number of plaquettes. Each operator $W_{p}$ has eigenvalues +1 and -1 , therefore each sector corresponds to a choice of $w_{p}= \pm 1$ for each plaquette $p$. Then we need to solve for the eigenvalues of the Hamiltonian restricted to a particular sector $\mathcal{L}_{w_{1}, \ldots w_{m}}$.

The honeycomb lattice has $1 / 2$ plaquette per vertex, therefore $m \approx n / 2$, where $n$ is the number of vertices. It follows that the dimension of each sector is $\sim 2^{n} / 2^{m} \sim 2^{n / 2}$ (we will in fact see that all these dimensions are equal). Thus splitting into sectors does not solve the problem yet. Fortunately, it turns out that the degrees of freedom within each sector can be described as real (Majorana) fermions, and the restricted Hamiltonian is simply a quadratic form in Majorana operators. This makes an exact solution possible.

\section{Representing spins by Majorana operators}

\subsection{A general spin-fermion transformation}

Let us remind the reader some general formalism pertaining to Fermi systems. A system with $n$ fermionic modes is usually described by the annihilation and creation operators $a_{k}, a_{k}^{\dagger}$ $(k=1, \ldots, n)$. Instead, one can use their linear combinations,

$$
c_{2 k-1}=a_{k}+a_{k}^{\dagger}, \quad c_{2 k}=\frac{a_{k}-a_{k}^{\dagger}}{i}
$$

which are called Majorana operators. The operators $c_{j}(j=1, \ldots, 2 n)$ are Hermitian and obey the following relations:

$$
c_{j}^{2}=1, \quad c_{j} c_{l}=-c_{l} c_{j} \text { if } j \neq l .
$$

Note that all operators $c_{j}$ can be treated on equal basis.

We now describe a representation of a spin by two fermionic modes, i.e., by four Majorana operators. Let us denote these operators by $b^{x}, b^{y}, b^{z}$, and $c$ (instead of $c_{1}, c_{2}, c_{3}, c_{4}$ ). The Majorana operators act on the 4-dimensional Fock space $\widetilde{\mathcal{M}}$, whereas the Hilbert space of a spin is identified with a two-dimensional subspace $\mathcal{M} \subset \widetilde{\mathcal{M}}$ defined by this condition:

$$
|\xi\rangle \in \mathcal{M} \quad \text { if and only if } \quad D|\xi\rangle=|\xi\rangle, \quad \text { where } D=b^{x} b^{y} b^{z} c .
$$

We call $\mathcal{M}$ and $\widetilde{\mathcal{M}}$ the physical subspace and the extended space, respectively; the operator $D$ may be thought of as a gauge transformation for the group $\mathbb{Z}_{2}$.

The Pauli operator $\sigma^{x}, \sigma^{y}, \sigma^{z}$ can be represented by some operators $\tilde{\sigma}^{x}, \tilde{\sigma}^{y}, \tilde{\sigma}^{z}$ acting on the extended space. Such a representation must satisfy two conditions: (1) $\widetilde{\sigma}^{x}, \widetilde{\sigma}^{y}, \widetilde{\sigma}^{z}$ preserve 
the subspace $\mathcal{M} ;(2)$ when restricted to $\mathcal{M}$, the operators $\tilde{\sigma}^{x}, \tilde{\sigma}^{y}, \tilde{\sigma}^{z}$ obey the same algebraic relations as $\sigma^{x}, \sigma^{y}, \sigma^{z}$. We will use the following particular representation:

$$
b_{\bullet}^{b^{\mathrm{x}}} \stackrel{\bullet}{c}_{b^{\mathrm{y}}} \quad \tilde{\sigma}^{x}=i b^{x} c, \quad \tilde{\sigma}^{y}=i b^{y} c, \quad \tilde{\sigma}^{z}=i b^{z} c
$$

(We have associated the Majorana operator with four dots for a reason that will be clear later.) This representation is correct since $\widetilde{\sigma}^{\alpha}(\alpha=x, y, z)$ commutes with $D$ (so that $\mathcal{M}$ is preserved), $\left(\widetilde{\sigma}^{\alpha}\right)^{\dagger}=\widetilde{\sigma}^{\alpha},\left(\widetilde{\sigma}^{\alpha}\right)^{2}=1$, and

$$
\tilde{\sigma}^{x} \widetilde{\sigma}^{y} \widetilde{\sigma}^{z}=i b^{x} b^{y} b^{z} c=i D .
$$

The last equation is consistent with the formula $\sigma^{x} \sigma^{y} \sigma^{z}=i$ because $D$ acts as the identity operator on the subspace $\mathcal{M}$.

A multi-spin system is described by four Majorana operators per spin. The corresponding operators $\widetilde{\sigma}^{\alpha_{j}}, D_{j}^{\alpha}$ and the physical subspace $\mathcal{L} \subset \widetilde{\mathcal{L}}$ are defined as follows:

$$
\begin{gathered}
\tilde{\sigma}^{\alpha_{j}}=i b_{j}^{\alpha} c_{j}, \quad D_{j}=b_{j}^{x} b_{j}^{y} b_{j}^{z} c_{j} \\
|\xi\rangle \in \mathcal{L} \quad \text { if and only if } \quad D_{j}|\xi\rangle=|\xi\rangle \text { for all } j .
\end{gathered}
$$

Any spin Hamiltonian $H\left\{\sigma_{j}^{\alpha}\right\}$ can be replaced by the fermionic Hamiltonian $\widetilde{H}\left\{b_{j}^{\alpha}, c_{j}\right\}=$ $H\left\{\widetilde{\sigma}^{\alpha_{j}}\right\}$ the action of which is restricted to the physical subspace. (The resulting Hamiltonian $\widetilde{H}$ is rather special; in particular, it commutes with the operators $D_{j}$.)

Remark 2.1. The substitution $\sigma_{j}^{\alpha} \mapsto \widetilde{\sigma}_{j}^{\alpha}=i b_{j}^{\alpha} c_{j}$ is gauge-equivalent to a more familiar one (see [49] and references therein):

$$
\sigma_{j}^{\alpha} \mapsto D_{j} \widetilde{\sigma}_{j}^{\alpha}, \quad \text { i.e., } \quad \sigma_{j}^{x} \mapsto-i b_{j}^{y} b_{j}^{z}, \quad \sigma_{j}^{y} \mapsto-i b_{j}^{z} b_{j}^{x}, \quad \sigma_{j}^{z} \mapsto-i b_{j}^{x} b_{j}^{y}
$$

Thus one can represent a spin by only 3 Majorana operators without imposing gauge constraints. However, this is not sufficient for our purposes.

\subsection{Application to the concrete model}

Let us apply the general procedure to the the spin Hamiltonian (44). Each term $K_{j k}=\sigma_{j}^{\alpha} \sigma_{k}^{\alpha}$ becomes $\widetilde{K}_{j k}=\left(i b_{j}^{\alpha} c_{j}\right)\left(i b_{k}^{\alpha} c_{k}\right)=-i\left(i b_{j}^{\alpha} b_{k}^{\alpha}\right) c_{j} c_{k}$. The operator in parentheses, $\hat{u}_{j k}=i b_{j}^{\alpha} b_{k}^{\alpha}$, is Hermitian; we associated it with the link $(j, k)$. (The index $\alpha$ takes values $x, y$ or $z$ depending on the direction of the link, i.e., $\alpha=\alpha_{j k}$.) Thus we get:

$$
\begin{aligned}
\widetilde{H}=\frac{i}{4} \sum_{j, k} \hat{A}_{j k} c_{j} c_{k}, \quad \hat{A}_{j k}=\left\{\begin{array}{cc}
2 J_{\alpha_{j k}} \hat{u}_{j k} & \text { if } j \text { and } k \text { are connected } \\
0 & \text { otherwise }
\end{array}\right. \\
\hat{u}_{j k}=i b_{j}^{\alpha_{j k}} b_{k}^{\alpha_{j k}} .
\end{aligned}
$$

Note that each pair of connected sites is counted twice, and $\hat{u}_{k j}=-\hat{u}_{j k}$. The structure of this Hamiltonian is shown in Fig. 4. 


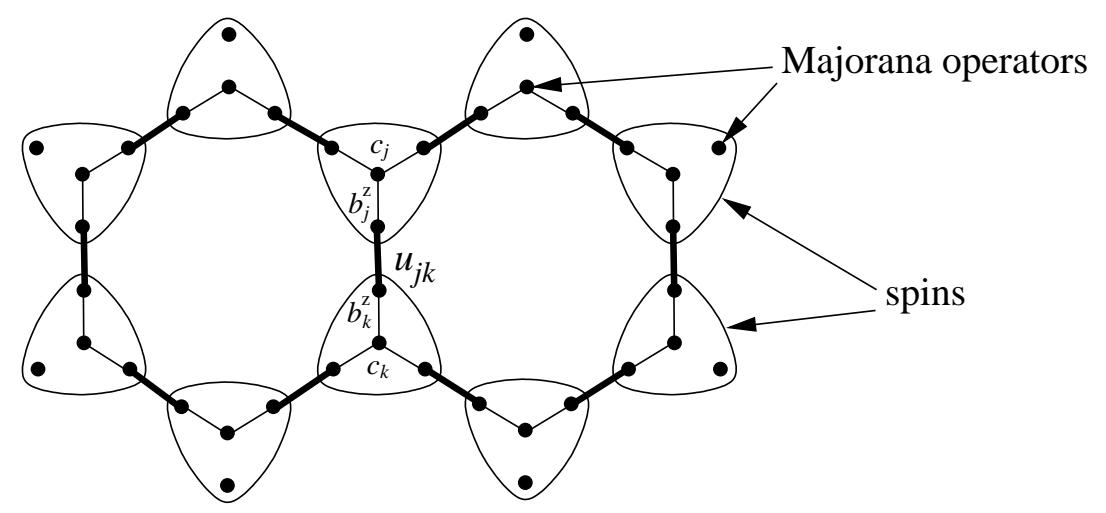

Figure 4: Graphic representation of Hamiltonian (13).

Remarkably, the operators $\hat{u}_{j k}$ commute with the Hamiltonian and with each other. Therefore the Hilbert space $\widetilde{\mathcal{L}}$ splits into common eigenspaces of $\hat{u}_{j k}$, which are indexed by the corresponding eigenvalues $u_{j k}= \pm 1$. Similarly to (7) we may write $\widetilde{\mathcal{L}}=\bigoplus_{u} \widetilde{\mathcal{L}}_{u}$, where $u$ stands for the collection of all $u_{j k}$. The restriction of Hamiltonian (13) to the subspace $\widetilde{\mathcal{L}}_{u}$ is obtained by "removing hats", i.e., replacing operators by numbers. This procedure results in the Hamiltonian $\widetilde{H}_{u}=\frac{i}{4} \sum_{j, k} A_{j k} c_{j} c_{k}$, which corresponds to free fermions. The ground state of $\widetilde{H}_{u}$ can be found exactly; let us denote it by $\left|\widetilde{\Psi}_{u}\right\rangle$.

Note, however, that the subspace $\widetilde{\mathcal{L}}_{u}$ is not gauge-invariant: applying the gauge operator $D_{j}$ changes the values of $u_{j k}$ on the links connecting the vertex $j$ with three adjacent vertices $k$. Therefore the state $\left|\widetilde{\Psi}_{u}\right\rangle$ does not belong to the physical subspace. To obtain a physical space we must symmetrize over all gauge transformations. Specifically, we construct the following state:

$$
\left|\Psi_{w}\right\rangle=\prod_{j}\left(\frac{1+D_{j}}{2}\right)\left|\widetilde{\Psi}_{u}\right\rangle \in \mathcal{L} .
$$

Here $w$ denotes the equivalence class of $u$ under the gauge transformations. For the planar lattice (but not on the torus) $w$ is characterized by numbers $w_{p}= \pm 1$ defined as products of $u_{j k}$ around hexagons. To avoid ambiguity (due to the relation $u_{k j}=-u_{j k}$ ), we choose a particular direction for each link:

$$
w_{p}=\prod_{(j, k) \in \operatorname{boundary}(p)} u_{j k} \quad(j \in \text { even sublattice, } k \in \text { odd sublattice }) .
$$

The corresponding operator $\widetilde{W}_{p}=\prod \hat{u}_{j k}$ commutes with the gauge transformations as well as the Hamiltonian. The restriction of this operator to the physical subspace coincides with the integral of motion $W_{p}$ defined earlier (see (6) ).

Notation change: From now on, we will not make distinction between operators acting in the extended space and their restrictions to the physical subspace e.g., $\widetilde{W}_{p}$ versus $W_{p}$. The tilde mark will be used for other purposes. 


\subsection{Path and loop operators}

One may think of the variables $u_{k j}$ as a $\mathbb{Z}_{2}$ gauge field. The number $w_{p}$ is interpreted as the magnetic flux through the plaquette $p$. If $w_{p}=-1$, we say that the plaquette carries a vortex.

The product of $\hat{u}_{j k}$ along an arbitrary path corresponds to the transfer of a fermion between the initial and the final point. However, this product is not gauge-invariant. One can define an invariant fermionic path operator in terms of spins or in terms of fermions:

$$
W\left(j_{0}, \ldots, j_{n}\right)=K_{j_{n} j_{n-1}} \ldots K_{j_{1} j_{0}}=\left(\prod_{s=1}^{n}-i \hat{u}_{j_{s} j_{s-1}}\right) c_{n} c_{0},
$$

where $K_{j k}$ is given by (515). If the path is closed, i.e., $j_{n}=j_{0}$, the factors $c_{n}$ and $c_{0}$ cancel each other. In this case the path operator is called the Wilson loop; it generalizes the notion of magnetic flux.

On the honeycomb lattice all loops have even length, and formula (16) agrees with the sign convention based on the partition into the even and odd sublattice. However, the spin model can be generalized to any trivalent graph, in which case the loop length is arbitrary. For an odd loop $l$ the operator $W(l)$ has eigenvalues $w_{l}= \pm i$. This is not just an artifact of the definition: odd loops are special in that they cause spontaneous breaking of the time-reversal symmetry.

The time-reversal operator is a conjugate-linear unitary operator $T$ such that

$$
T \sigma_{j}^{\alpha} T^{-1}=-\sigma_{j}^{\alpha}, \quad T b_{j} T^{-1}=b_{j}, \quad T c_{j} T^{-1}=c_{j} .
$$

(The first equation is a physical requirement; the other two represent the action of $T$ in the extended space.) Therefore $T$ commutes with the Hamiltonian (4) and the Wilson loop. Multiplying the equation $W_{l}|\Psi\rangle=w_{l}|\Psi\rangle$ by $T$, we get $W_{l} T|\Psi\rangle=w_{l}^{*} T|\Psi\rangle$. Thus the time-reversal operator changes $w_{l}$ to $w_{l}^{*}$. For a bipartite graph $w_{l}^{*}=w_{l}$ for all loops, therefore fixing the variables $w_{l}$ does not break the time-reversal symmetry. On the contrary, for a similar model on a non-bipartite graph (e.g., a lattice containing triangles) the operator $T$ does not preserve the field configuration, which is defined by the values of $w_{l}$ on all loops. But $T$ is a symmetry operator, therefore all Hamiltonian eigenstates are (at least) two-fold degenerate.

\section{Quadratic Hamiltonians}

In the previous section we transformed the spin model (4) to a quadratic fermionic Hamiltonian of the general form

$$
H(A)=\frac{i}{4} \sum_{j, k} A_{j k} c_{j} c_{k},
$$

where $A$ is a real skew-symmetric matrix of size $n=2 m$. Let us briefly state some general properties of such Hamiltonians and fix the terminology.

First, we comment on the normalization factor $1 / 4$ in Eq. (18). It is chosen so that

$$
[-i H(A),-i H(B)]=-i H([A, B]) .
$$

Thus the Lie algebra of quadratic operators $-i H(A)$ (acting on the $2^{m}$-dimensional Fock space) is identified with $\mathfrak{s o}(2 m)$. Operators of the form $e^{-i H(A)}$ constitute the Lie group $\operatorname{Spin}(2 m)$. 
The center of this group consists of phase factors \pm 1 (e.g., $e^{\pi c_{1} c_{2}}=-1$ ). The quotient group $\operatorname{Spin}(2 m) /\{+1,-1\}=\mathrm{SO}(2 m)$ describes the action of $e^{-i H(A)}$ on Majorana operators by conjugation:

$$
e^{-i H(A)} c_{k} e^{i H(A)}=\sum_{j} Q_{j k} c_{j}, \quad \text { where } Q=e^{A}
$$

( $Q$ is a real orthogonal matrix with determinant 1$)$.

Note that the sum in Eq. (20) corresponds to the multiplication of the row vector $\left(c_{1}, \ldots, c_{2 m}\right)$ by the matrix $Q$. On the other hand, when we consider a linear combination of Majorana operators,

$$
F(x)=\sum_{j} x_{j} c_{j}
$$

we prefer to view $x$ as a column vector. If the coefficients $x_{j}$ are real, we call $F(x)$ (or $x$ itself) a Majorana mode.

To find the ground state of the Hamiltonian (18), one needs to reduce it to a canonical form

$$
H_{\text {canonical }}=\frac{i}{2} \sum_{k=1}^{m} \varepsilon_{k} b_{k}^{\prime} b_{k}^{\prime \prime}=\sum_{k=1}^{m} \varepsilon_{k}\left(a_{k}^{\dagger} a_{k}-\frac{1}{2}\right), \quad \varepsilon_{k} \geqslant 0,
$$

where $b_{k}^{\prime}, b_{k}^{\prime \prime}$ are normal modes, and $a_{k}^{\dagger}=\frac{1}{2}\left(b_{k}^{\prime}-i b_{k}^{\prime \prime}\right), a_{k}=\frac{1}{2}\left(b_{k}^{\prime}+i b_{k}^{\prime \prime}\right)$ are the corresponding creation and annihilation operators. The ground state of $H_{\text {canonical }}$ is characterized by the condition $a_{k}|\Psi\rangle=0$ for all $k$. The reduction to the canonical form is achieved by the transformation

$$
\left(b_{1}^{\prime}, b_{1}^{\prime \prime}, \ldots, b_{m}^{\prime}, b_{m}^{\prime \prime}\right)=\left(c_{1}, c_{2}, \ldots, c_{2 m-1}, c_{2 m}\right) Q, \quad Q \in \mathrm{O}(2 m)
$$

such that

$$
A=Q\left(\begin{array}{ccccc}
0 & \varepsilon_{1} & & & \\
-\varepsilon_{1} & 0 & & & \\
& & \ddots & & \\
& & & 0 & \varepsilon_{m} \\
& & & -\varepsilon_{m} & 0
\end{array}\right) Q^{T}
$$

The numbers $\pm \varepsilon_{k}$ constitute the spectrum of the Hermitian matrix $i A$, whereas odd (even) columns of $Q$ are equal to the real (resp., imaginary) part of the eigenvectors. The ground state of the Majorana system has energy

$$
E=-\frac{1}{2} \sum_{k=1}^{m} \varepsilon_{k}=-\frac{1}{4} \operatorname{Tr}|i A|,
$$

where the function $|\cdot|$ acts on the eigenvalues, the eigenvectors being fixed. (In fact, any function of a real variable can be applied to Hermitian matrices.)

Note that different quadratic Hamiltonians may give rise to the same ground state. The latter actually depends on

$$
B=-i \operatorname{sgn}(i A)=Q\left(\begin{array}{ccccc}
0 & 1 & & & \\
-1 & 0 & & & \\
& & \ddots & & \\
& & & 0 & 1 \\
& & & -1 & 0
\end{array}\right) Q^{T}
$$


(We assume that $A$ is not degenerate.) The matrix $B$ is real skew-symmetric and satisfies $B^{2}=-1$. It determines the ground state through the condition

$$
\sum_{j} P_{j k} c_{j}|\Psi\rangle=0 \quad \text { for all } k, \quad \text { where } P_{j k}=\frac{1}{2}\left(\delta_{j k}-i B_{j k}\right) \text {. }
$$

Loosely speaking, $B$ corresponds to a pairing ${ }^{1}$ between Majorana modes. The operators $b^{\prime}=$ $F\left(x^{\prime}\right)$ and $b^{\prime \prime}=F\left(x^{\prime \prime}\right)$ are paired if $x^{\prime \prime}= \pm B x^{\prime}$.

The matrix $P$ in Eq. (27) is called the spectral projector. It projects the $2 m$-dimensional complex space $\mathbb{C}^{2 m}$ onto the $m$-dimensional subspace $L$ spanned by the eigenvectors of $i A$ corresponding to negative eigenvalues. For any vector $z \in L$ the corresponding operator $F(z)$ annihilates the ground state, so we may call $L$ the space of annihilation operators. Note that if $z, z^{\prime} \in L$ then $\sum_{j} z_{j} z_{j}^{\prime}=0$. The choice of an $m$-dimensional subspace $L \subseteq \mathbb{C}^{2 m}$ satisfying this condition is equivalent to the choice of matrix $B$.

The ground state of a quadratic Hamiltonian can also be characterized by correlation functions. The second-order correlator is $\left\langle\Psi\left|c_{j} c_{k}\right| \Psi\right\rangle=2 P_{k j}$; higher-order correlators can be found using Wick's formula.

\section{The spectrum of fermions and the phase diagram}

We now study the system of Majorana fermions on the honeycomb lattice. It is described by the quadratic Hamiltonian $H_{u}=H(A)$, where $A_{j k}=2 J_{\alpha_{j k}} u_{j k}, u_{j k}= \pm 1$. Although the Hamiltonian is parametrized by $u_{j k}$, the corresponding gauge-invariant state (or the state of the spin system) actually depends on the variables $w_{p}$, see (15).

First, we remark that the global ground state energy does not depend on the signs of the exchange constants $J_{x}, J_{y}, J_{z}$ since changing the signs can be compensated by changing the corresponding variables $u_{j k}$. We further notice that the ground state energy for $H_{u}$ does not depend on these signs even if $u$ is fixed. Suppose, for instance, that we replace $J_{z}$ by $-J_{z}$. Such a change is equivalent to altering $u_{j k}$ for all $z$-links. But the gauge-invariant quantities $w_{p}$ remain constant, so we may apply a gauge transformation that returns $u_{j k}$ to their original values. The net effect is that the Majorana operators at some sites are transformed as $c_{j} \mapsto-c_{j}$. Specifically, the transformation acts on the set of sites $\Omega_{z}$ that lie in the shaded area in the picture below. In terms of spins, this action is induced by the unitary operator

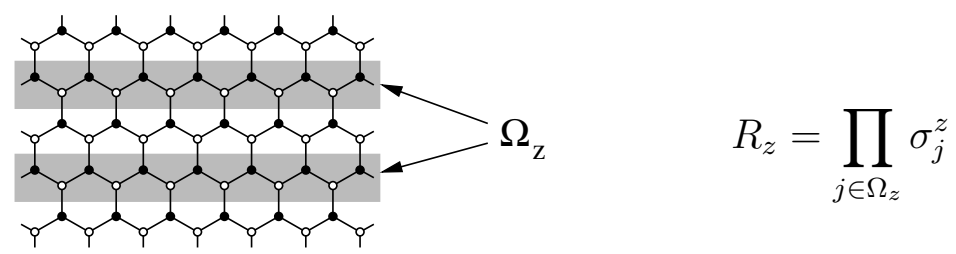

So, for the purpose of finding the ground state energy and the excitation spectrum, the signs of exchange constants do not matter (but other physical quantities may depend on them).

\footnotetext{
${ }^{1}$ Note the nonstandard use of terminology: in our sense, electron half-modes in an insulator are paired as well as in a superconductor. The only difference is that the insulating pairing commutes with the number of electrons while the superconducting one doesn't. This distinction is irrelevant to our model because the Hamiltonian doesn't preserve any integral charge, and the number of fermions is only conserved modulo 2.
} 
The most interesting choice of $u_{j k}$ is the one that minimizes the ground state energy. It turns out that the energy minimum is achieved by the vortex-free field configuration, i.e., $w_{p}=1$ for all plaquettes $p$. This statement follows from a beautiful theorem proved by Lieb [50]. (Not knowing about Lieb's result, I did some numerical study suggesting the same answer, see Appendix A.) Thus we may assume that $u_{j k}=1$ for all links $(j, k)$, where $j$ belongs to the even sublattice, and $k$ belongs to the odd sublattice. This field configuration (denoted by $u_{j k}^{\text {std }}$ ) possesses a translational symmetry, therefore the fermionic spectrum can be found analytically using the Fourier transform.

The general procedure is as follows. Let us represent the site index $j$ as $(s, \lambda)$, where $s$ refers to a unit cell, and $\lambda$ to a position type inside the cell (we choose the unit cell as shown in the figure accompanying Eq. (32) ). The Hamiltonian becomes $H=(i / 4) \sum_{s, \lambda, t, \mu} A_{s \lambda, t \mu} c_{s \lambda} c_{t \mu}$, where $A_{s \lambda, t \mu}$ actually depends on $\lambda, \mu$, and $t-s$. Then we pass to the momentum representation:

$$
\begin{gathered}
H=\frac{1}{2} \sum_{\mathbf{q}, \lambda, \mu} i \widetilde{A}_{\lambda \mu}(\mathbf{q}) a_{-\mathbf{q}, \lambda} a_{\mathbf{q}, \mu}, \quad \widetilde{A}_{\lambda \mu}(\mathbf{q})=\sum_{t} e^{i\left(\mathbf{q}, \mathbf{r}_{t}\right)} A_{0 \lambda, t \mu} \\
a_{\mathbf{q}, \lambda}=\frac{1}{\sqrt{2 N}} \sum_{s} e^{-i\left(\mathbf{q}, \mathbf{r}_{s}\right)} c_{s \lambda}
\end{gathered}
$$

where $N$ is the total number of the unit cells. (Here and on, operators in the momentum representation are marked with tilde.) Note that $a_{\mathbf{q}, \lambda}^{\dagger}=a_{-\mathbf{q}, \lambda}$ and $a_{\mathbf{p}, \mu} a_{\mathbf{q}, \lambda}^{\dagger}+a_{\mathbf{q}, \lambda}^{\dagger} a_{\mathbf{p}, \mu}=\delta_{\mathbf{p q}} \delta_{\mu \lambda}$. The spectrum $\varepsilon(\mathbf{q})$ is given by the eigenvalues of the matrix $i \widetilde{A}(\mathbf{q})$. One may call it a "double spectrum" because of its redundancy: $\varepsilon(-\mathbf{q})=-\varepsilon(-\mathbf{q})$. The "single spectrum" can be obtained by taking only positive eigenvalues (if none of the eigenvalues is zero).

We now apply this procedure to the concrete Hamiltonian

$$
H_{\mathrm{vortex}-\mathrm{free}}=\frac{i}{4} \sum_{j, k} A_{j k} c_{j} c_{k}, \quad A_{j k}=2 J_{\alpha_{j k}} u_{j k}^{\mathrm{std}}
$$

We choose a basis $\left(\mathbf{n}_{1}, \mathbf{n}_{2}\right)$ of the translation group and obtain the following result:

$$
\begin{gathered}
i \widetilde{A}(\mathbf{q})=\left(\begin{array}{cc}
0 & i f(\mathbf{q}) \\
-i f(\mathbf{q})^{*} & 0
\end{array}\right), \quad \varepsilon(\mathbf{q})= \pm|f(\mathbf{q})|, \\
f(\mathbf{q})=2\left(J_{x} e^{i\left(\mathbf{q}, \mathbf{n}_{1}\right)}+J_{y} e^{i\left(\mathbf{q}, \mathbf{n}_{2}\right)}+J_{z}\right),
\end{gathered}
$$

where $\mathbf{n}_{1}=\left(\frac{1}{2}, \frac{\sqrt{3}}{2}\right), \mathbf{n}_{2}=\left(-\frac{1}{2}, \frac{\sqrt{3}}{2}\right)$ in the standard $x y$-coordinates.

An important property of the spectrum is whether it is gapless, i.e., whether $\varepsilon(\mathbf{q})$ is zero for some $\mathbf{q}$. The equation $J_{x} e^{i\left(\mathbf{q}, \mathbf{n}_{1}\right)}+J_{y} e^{i\left(\mathbf{q}, \mathbf{n}_{2}\right)}+J_{z}=0$ has solutions if and only if $\left|J_{x}\right|,\left|J_{y}\right|$, $\left|J_{z}\right|$ satisfy the triangle inequalities:

$$
\left|J_{x}\right| \leqslant\left|J_{y}\right|+\left|J_{z}\right|, \quad\left|J_{y}\right| \leqslant\left|J_{x}\right|+\left|J_{z}\right|, \quad\left|J_{z}\right| \leqslant\left|J_{x}\right|+\left|J_{y}\right| .
$$

If the inequalities are strict (" $<$ " instead of " $\leqslant$ "), there are exactly 2 solutions: $\mathbf{q}= \pm \mathbf{q}_{*}$. The region defined by inequalities (33) is marked by $B$ in Fig. 5 this phase is gapless. The 


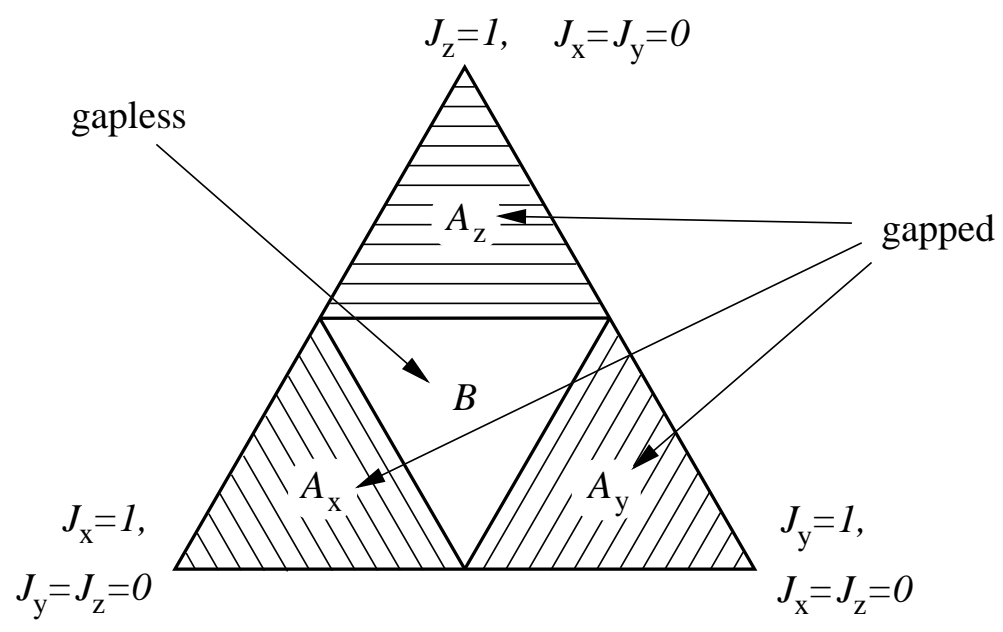

Figure 5: Phase diagram of the model. The triangle is the section of the positive octant $\left(J_{x}, J_{y}, J_{z} \geqslant 0\right)$ by the plane $J_{x}+J_{y}+J_{z}=1$. The diagrams for the other octants are similar.

gapped phases, $A_{x}, A_{y}$, and $A_{z}$, are algebraically distinct, though related to each other by rotational symmetry. They differ in the way lattice translations act on anyonic states (see Section 5.21). Therefore a continuous transition from one gapped phase to another is impossible, even if we introduce new terms in the Hamiltonian. On the other hand, the 8 copies of each phase (corresponding to different sign combinations of $J_{x}, J_{y}, J_{z}$ ) have the same translational properties. It is unknown whether the 8 copies of the gapless phase are algebraically different.

We now consider the zeros of the spectrum that exist in the gapless phase. The momentum $\mathbf{q}$ is defined modulo the reciprocal lattice, i.e., it belongs to a torus. We represent the momentum space by the parallelogram spanned by $\left(\mathbf{q}_{1}, \mathbf{q}_{2}\right)$ - the basis dual to $\left(\mathbf{n}_{1}, \mathbf{n}_{2}\right)$. In the symmetric case $\left(J_{x}=J_{y}=J_{z}\right)$ the zeros of the spectrum are given by

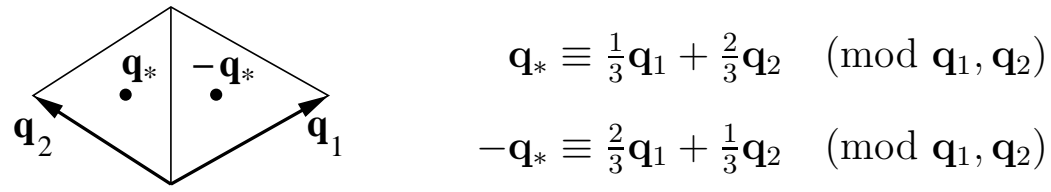

If $\left|J_{x}\right|$ and $\left|J_{y}\right|$ decrease while $\left|J_{z}\right|$ remains constant, $\mathbf{q}_{*}$ and $-\mathbf{q}_{*}$ move toward each other (within the parallelogram) until they fuse and disappear. This happens when $\left|J_{x}\right|+\left|J_{y}\right|=\left|J_{z}\right|$. The points $\mathbf{q}_{*}$ and $-\mathbf{q}_{*}$ can also effectively fuse at opposite sides of the parallelogram. (Note that the equation $\mathbf{q}_{*}=-\mathbf{q}_{*}$ has three nonzero solutions on the torus).

At the points $\pm \mathbf{q}_{*}$ the spectrum has conic singularities (assuming that $\mathbf{q}_{*} \neq-\mathbf{q}_{*}$ ):

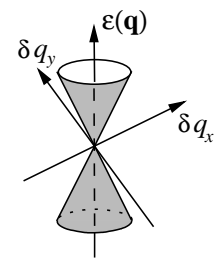

$$
\varepsilon(\mathbf{q}) \approx \pm \sqrt{g_{\alpha \beta} \delta q_{\alpha} \delta q_{\beta}},
$$

where $\delta \mathbf{q}=\mathbf{q}-\mathbf{q}_{*}$ or $\delta \mathbf{q}=\mathbf{q}+\mathbf{q}_{*}$. 


\section{$5 \quad$ Properties of the gapped phases}

In a gapped phase, spin correlations decay exponentially with distance, therefore spatially separated quasiparticles cannot interact directly. That is, a small displacement or another local action on one particle does not influence the other. However, the particles can interact topologically if they move around each other. This phenomenon is described by braiding rules. (We refer to braids that are formed by particle worldlines in the 3-dimensional space-time.) In our case the particles are vortices and fermions. When a fermion moves around a vortex, the overall quantum state is multiplied by -1 . As mentioned in the introduction, such particles (with braiding characterized simply by phase factors) are called Abelian anyons.

The description of anyons begins with identifying superselection sectors, i.e., excitation types defined up to local operations. (An "excitation" is assumed to be localized in space, but it may have uncertain energy or be composed of several unbound particles.) The trivial superselection sector is that of the vacuum; it also contains all excitations that can be obtained from the vacuum by the action of local operators.

At first sight, each gapped phase in our model has three superselection sectors: a fermion, a vortex and the vacuum. However, we will see that there are actually two types of vortices that live on different subsets of plaquettes. They have the same energy and other physical characteristics, yet they belong to different superselection sectors: to transform one type of vortex into the other one has to create or annihilate a fermion.

To understand the particle types and other algebraic properties of the gapped phases, we will map our model to an already known one [24]. Let us focus on the phase $A_{z}$, which occurs when $\left|J_{x}\right|+\left|J_{y}\right|<\left|J_{z}\right|$. Since we are only interested in discrete characteristics of the phase, we may set $\left|J_{x}\right|,\left|J_{y}\right| \ll\left|J_{z}\right|$ and apply the perturbation theory.

\subsection{Perturbation theory study}

The Hamiltonian is $H=H_{0}+V$, where $H_{0}$ is the main part and $V$ is the perturbation:

$$
H_{0}=-J_{z} \sum_{z \text {-links }} \sigma_{j}^{z} \sigma_{k}^{z}, \quad V=-J_{x} \sum_{x \text {-links }} \sigma_{j}^{x} \sigma_{k}^{x}-J_{y} \sum_{y \text {-links }} \sigma_{j}^{y} \sigma_{k}^{y}
$$

Let us assume that $J_{z}>0$ (the opposite case is studied analogously).

We first set $J_{x}=J_{y}=0$ and find the ground state. It is highly degenerate: each two spins connected by a $z$-link are aligned $(\uparrow \uparrow$ or $\downarrow \downarrow)$, but their common direction is not fixed. We regard each such pair as an effective spin. The transition from physical spins to effective spins is shown in Fig. 6a,b. The ground state energy is $E_{0}=-N J_{z}$, where $N$ is the number of unit cells, i.e., half the number of spins.

Our goal is to find an effective Hamiltonian that would act in the space of effective spins $\mathcal{L}_{\text {eff }}$. One way to solve the problem is to choose a basis in $\mathcal{L}_{\text {eff }}$ and compute the matrix elements

$$
\left\langle a\left|H_{\mathrm{eff}}^{(1)}\right| b\right\rangle=\langle a|V| b\rangle, \quad\left\langle a\left|H_{\mathrm{eff}}^{(2)}\right| b\right\rangle=\sum_{j}^{\prime} \frac{\langle a|V| j\rangle\langle j|V| b\rangle}{E_{0}-E_{j}}, \quad \text { etc. }
$$

However, we will use the more general Green function formalism.

Let $\Upsilon: \mathcal{L}_{\text {eff }} \rightarrow \mathcal{L}$ be the embedding that maps the effective Hilbert space onto the ground subspace of $H_{0}$. The map $\Upsilon$ simply doubles each spin: $\Upsilon|m\rangle=|m m\rangle$, where $m=\uparrow$ or 


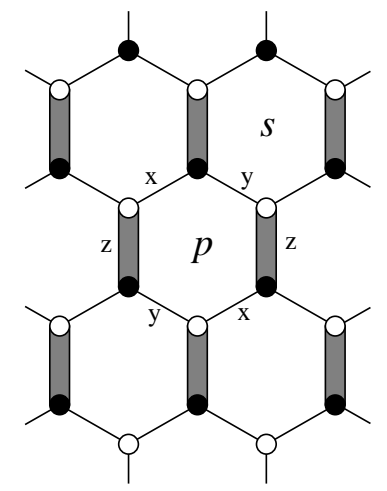

a)

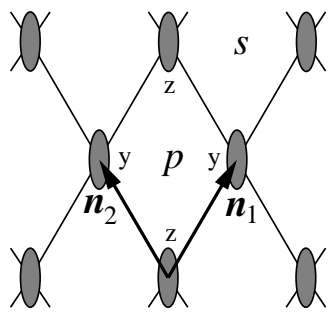

b)

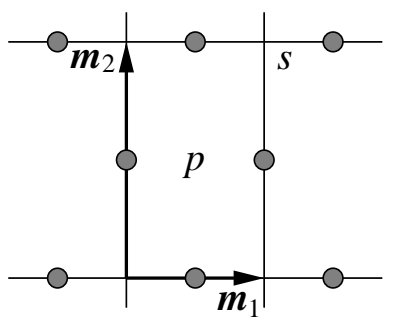

c)

Figure 6: Reduction of the model. Strong links in the original model (a) become effective spins (b), which are associated with the links of a new lattice (c).

$m=\downarrow$. The eigenvalues of the "effective Hamiltonian" (if one exists) are supposed to be the energy levels of $H$ that originate from ground states of $H_{0}$. These levels can be unambiguously defined as poles of the Green function $G(E)=\Upsilon^{\dagger}(E-H)^{-1} \Upsilon$, which is an operator acting on $\mathcal{L}_{\text {eff }}$ and depending on the parameter $E$. The Green function is conventionally expressed as $\left(E-E_{0}-\Sigma(E)\right)^{-1}$, where $\Sigma(E)$ is called self-energy, so the energy levels in question are the values of $E$ for which the operator $E-E_{0}-\Sigma(E)$ is degenerate. Neglecting the the dependence of $\Sigma(E)$ on $E$ (for $E \approx E_{0}$ ), we define the effective Hamiltonian as $H_{\text {eff }}=E_{0}+\Sigma\left(E_{0}\right)$.

The self-energy is computed by the standard method. Let $G_{0}^{\prime}(E)=\left(\left(E-H_{0}\right)^{-1}\right)^{\prime}$ be the unperturbed Green function for exited states of $H_{0}$. The ' sign indicates that the operator $\left(\left(E-H_{0}\right)^{-1}\right)^{\prime}$ acts on excited states in the natural way but vanishes on ground states. Then

$$
\Sigma(E)=\Upsilon^{\dagger}\left(V+V G_{0}^{\prime}(E) V+V G_{0}^{\prime}(E) V G_{0}^{\prime}(E) V+\cdots\right) \Upsilon
$$

We set $E=E_{0}$ and compute $H_{\text {eff }}=E_{0}+\Sigma$ in the zeroth order $\left(H_{\text {eff }}^{(0)}=E_{0}\right)$, first order, second order, and on, until we find a nonconstant term. ${ }^{2}$ The calculation follows.

1. $H_{\mathrm{eff}}^{(1)}=\Upsilon^{\dagger} V \Upsilon=0$.

2. $H_{\text {eff }}^{(2)}=\Upsilon^{\dagger} V G_{0}^{\prime} V \Upsilon=-\sum_{x \text {-links }} \frac{J_{x}^{2}}{4 J_{z}}-\sum_{y \text {-links }} \frac{J_{y}^{2}}{4 J_{z}}=-N \frac{J_{x}^{2}+J_{y}^{2}}{4 J_{z}}$. Indeed, consider the action of the second $V$ in the expression $\Upsilon^{\dagger} V G_{0}^{\prime} V \Upsilon$. Each term $\sigma_{j}^{x} \sigma_{k}^{x}$ or $\sigma_{j}^{y} \sigma_{k}^{y}$ flips two spins, increasing the energy by $4 J_{z}$. The other $V$ must flip them back.

3. $H_{\mathrm{eff}}^{(3)}=\Upsilon^{\dagger} V G_{0}^{\prime} V G_{0}^{\prime} V \Upsilon=0$.

4. $H_{\mathrm{eff}}^{(4)}=\Upsilon^{\dagger} V G_{0}^{\prime} V G_{0}^{\prime} V G_{0}^{\prime} V \Upsilon=\mathrm{const}-\frac{J_{x}^{2} J_{y}^{2}}{16 J_{z}^{3}} \sum_{p} Q_{p}$, where $Q_{p}=\left(W_{p}\right)_{\text {eff }}$ is the effective spin representation of the operator (6). The factor $\frac{1}{16}$ is obtained by summing 24 terms,

\footnotetext{
${ }^{2}$ Higher order terms may be less significant than the dependence of $\Sigma(E)$ on $E$ in the first nonconstant term.
} 
each of which corresponds to flipping 4 spin pairs in a particular order:

$$
\frac{1}{16}=8 \cdot \frac{1}{64}+8 \cdot \frac{-1}{64}+8 \cdot \frac{1}{128} \text {. }
$$

The above arguments can be easily adapted to the case $J_{z}<0$. Now we have $\Upsilon:|\uparrow\rangle \mapsto$ $|\uparrow \downarrow\rangle, \quad|\downarrow\rangle \mapsto|\downarrow \uparrow\rangle$. The result turns out to be the same, with $J_{z}$ replaced by $\left|J_{z}\right|$.

Thus the effective Hamiltonian has the form

$$
H_{\mathrm{eff}}=-\frac{J_{x}^{2} J_{y}^{2}}{16\left|J_{z}\right|^{3}} \sum_{p} Q_{p}, \quad Q_{p}=\sigma_{\text {left }(p)}^{y} \sigma_{\operatorname{right}(p)}^{y} \sigma_{\mathrm{up}(p)}^{z} \sigma_{\mathrm{down}(p)}^{z}
$$

(the geometric arrangement of the spins corresponds to Fig. 6b).

\subsection{Abelian anyons}

Hamiltonian (37) already lends itself to direct analysis. However, let us first reduce it to the more familiar form (3). We construct a new lattice $\Lambda^{\prime}$ so that the effective spins lie on its links (see Fig. 6c). This is a sublattice of index 2 in the original lattice $\Lambda$ (here "lattice" means "translational group"). The basis vectors of $\Lambda^{\prime}$ are $\mathbf{m}_{1}=\mathbf{n}_{1}-\mathbf{n}_{2}$ and $\mathbf{m}_{2}=\mathbf{n}_{1}+\mathbf{n}_{2}$. The plaquettes of the effective spin lattice become plaquettes and vertices of the new lattice, so the Hamiltonian can be written as follows:

$$
H_{\mathrm{eff}}=-J_{\mathrm{eff}}\left(\sum_{\text {vertices }} Q_{s}+\sum_{\text {plaquettes }} Q_{p}\right)
$$

where $J_{\text {eff }}=J_{x}^{2} J_{y}^{2} /\left(16\left|J_{z}\right|^{3}\right)$.

Now we apply the unitary transformation

$$
U=\prod_{\text {horizontal links }} X_{j} \prod_{\text {vertical links }} Y_{k}
$$

for suitably chosen spin rotations $X$ and $Y$ so that the Hamiltonian becomes

$$
H_{\mathrm{eff}}^{\prime}=U H_{\mathrm{eff}} U^{\dagger}=-J_{\mathrm{eff}}\left(\sum_{\text {vertices }} A_{s}+\sum_{\text {plaquettes }} B_{p}\right),
$$

where $A_{s}$ and $B_{p}$ are defined in Eq. (3). (Caution: transformation (38) breaks the translational symmetry of the original model.)

The last Hamiltonian has been studied in detail 24. Its key properties are that all the terms $A_{s}, B_{p}$ commute, and that the ground state minimizes each term separately. Thus the ground state satisfies these conditions:

$$
A_{s}|\Psi\rangle=+|\Psi\rangle, \quad B_{p}|\Psi\rangle=+|\Psi\rangle .
$$

Excited states can be obtained by replacing the + sign to a - sign for a few vertices and plaquettes. Those vertices and plaquettes are the locations of anyons. We call them "electric charges" 
and "magnetic vortices", or $e$-particles and $m$-particles, respectively. When an $e$-particle moves around a $m$-particle, the overall state of the system is multiplied by -1 . This property is stable with respect to small local perturbations of the Hamiltonian. (A local operator is a sum of terms each of which acts on a small number of neighboring spins.) It is also a robust property that the number of particles of each type is conserved modulo 2.

The model has 4 superselection sectors: 1 (the vacuum), $e, m$, and $\varepsilon=e \times m$. The latter expression denotes a composite object consisting of an "electric charge" and a "magnetic vortex". These are the fusion rules:

$$
\begin{gathered}
e \times e=m \times m=\varepsilon \times \varepsilon=1, \\
e \times m=\varepsilon, \quad e \times \varepsilon=m, \quad m \times \varepsilon=e .
\end{gathered}
$$

(In general, fusion rules must be supplemented by associativity relations, or $6 j$-symbols, but they are trivial in our case.)

Let us discuss the braiding rules. One special case has been mentioned: moving an $e$-particle around an $m$-particle yields -1 . This fact can be represented pictorially:

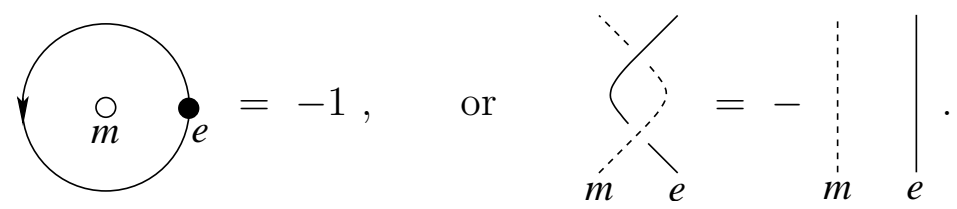

The fist diagram shows the "top view" of the process. The diagrams in the second equation correspond to the "front view": the "up" direction is time.

It is easy to show that $e$-particles are bosons with respect to themselves (though they clearly do not behave like bosons with respect to $m$-particles); $m$-particles are also bosons. However $\varepsilon$-particles are fermions. To see this, consider two processes. In the first process two $\varepsilon \varepsilon$-pairs are created, two of the four $\varepsilon$-objects are exchanged by a $180^{\circ}$ counterclockwise rotation, then the pairs are annihilated. (Each $\varepsilon$-object is represented by $e$ and $m$, so there are 8 elementary particles involved.) In the second process the two pairs are annihilated immediately. It does not matter how exactly we create and annihilate the pairs, but we should do it the same way in both cases. For example, we may use this definition:

$$
\text { creation }=\stackrel{m e}{m e}, \quad \text { annihilation }=\bigwedge_{m e}^{m e} .
$$

Now we compare the two processes. Each one effects the multiplication of the ground state by a number, but the two numbers differ by -1 :

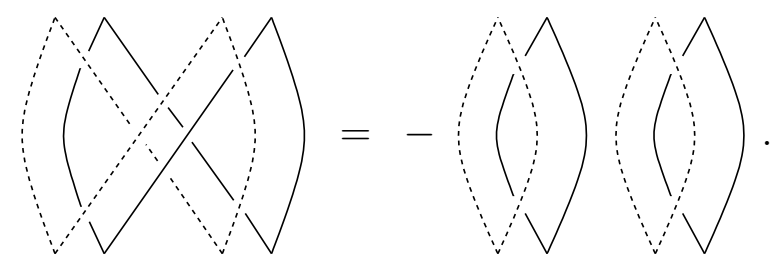

Indeed, in the left diagram the dashed line is linked with the solid line. This corresponds to an $e$-particle going around an $m$ particle, which yields the minus sign. 


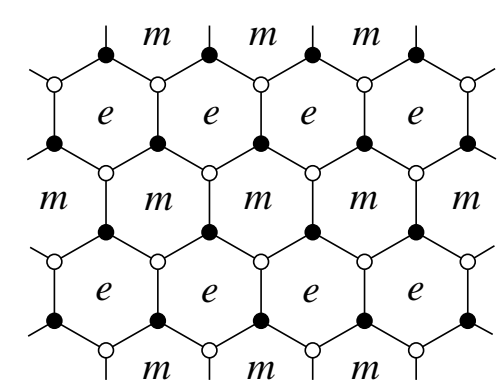

Figure 7: Weak breaking of the translational symmetry: $e$-vortices and $m$-vortices live on alternating rows of hexagons.

Braiding an $\varepsilon$-particle with an $e$ - or $m$-particle also gives -1 . This completes the description of braiding rules.

It remains to interpret these properties in terms of vortices and fermions in the original model. Tracing sites and plaquettes of the reduced model (31) back to the original model, we conclude that $e$-particles and $m$-particles are vortices that live on alternating rows of hexagons, see Fig. 7 Note that the two types of vortices have the same energy and other physical properties, yet they cannot be transformed one to another without creating or absorbing a fermion. A general view on this kind of phenomenon is given in Appendix $\mathbf{E}$

The fermions in the original model belong to the superselection sector $\varepsilon$, although they are not composed of $e$ and $m$. In the perturbation-theoretic limit, the energy of a fermion is about $2\left|J_{z}\right|$ whereas an $\mathrm{em}$-pair has energy $4 J_{\text {eff }} \ll\left|J_{z}\right|$. The fermions are stable due to the conservation of $W_{p}$ (and also due to the conservation of energy). However, they will decay into $e$ and $m$ if we let the spins interact with a zero-temperature bath, i.e., another system that can absorb the energy released in the decay.

\section{Phase $B$ acquires a gap in the presence of magnetic field}

\subsection{The conic singularity and the time-reversal symmetry}

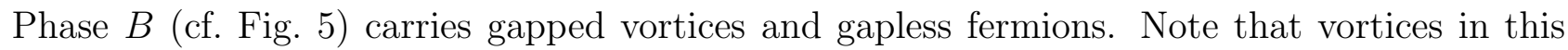
phase do not have well-defined statistics, i.e., the effect of transporting one vortex around the other depends on details of the process. Indeed, a pair of vortices separated by distance $L$ is strongly coupled to fermionic modes near the singularity of the spectrum, $\left|\mathbf{q}-\mathbf{q}_{*}\right| \sim L^{-1}$. This coupling results in effective interaction between the vortices that is proportional to $\varepsilon(\mathbf{q}) \sim L^{-1}$ and oscillates with characteristic wavevector $2 \mathbf{q}_{*}$. When one vortex moves around the other, the quantum state picks up a nonuniversal phase $\varphi \sim L^{-1} t$, where $t$ is the duration of the process. Since the vortex velocity $v=L / t$ must be small to ensure adiabaticity (or, at least, to prevent the emission of fermionic pairs), the nonuniversal phase $\varphi$ is always large.

The conic singularity in the spectrum is, in fact, a robust feature that is related to timereversal symmetry. As discussed in the end of Section 2, this symmetry is not broken by fixing the gauge sector (i.e., the variables $w_{p}$ ) since the honeycomb lattice is bipartite. Let us show 
that a small perturbation commuting with the time-reversal operator $T$ can not open a spectral gap.

We may perform the perturbation theory expansion relative to the gauge sector of the ground state (i.e., any term that changes the field configuration is taken into account in the second and higher orders). This procedure yields an effective Hamiltonian which acts in the fixed gauge sector and therefore can be represented in terms of $c_{j}$ and $\hat{u}_{j k}$. The operator $T$ defined by (17) changes the sign of $\hat{u}_{j k}=i b_{j}^{\alpha_{j k}} b_{k}^{\alpha_{j k}}$, but this change can be compensated by a gauge transformation. Thus we get a physically equivalent operator $T^{\prime}$ such that

$$
T^{\prime} \hat{u}_{j k}\left(T^{\prime}\right)^{-1}=\hat{u}_{j k}, \quad T^{\prime} c_{j}\left(T^{\prime}\right)^{-1}=\left\{\begin{array}{c}
c_{j} \text { if } j \in \text { even sublattice } \\
-c_{j} \text { if } j \in \text { odd sublattice. }
\end{array}\right.
$$

A $T^{\prime}$-invariant perturbation to the fermionic Hamiltonian (31) (corresponding to a fixed gauge) can not contain terms like $i c_{j} c_{k}$, where $j$ and $k$ belong to the same sublattice. Thus the perturbed matrix $\widetilde{A}(\mathbf{q})$ in Eq. (32) still has zeros on the diagonal, though the exact form of the function $f(\mathbf{q})$ may be different. However, a zero of a complex-valued function in two real variables is a topological feature, therefore it survives the perturbation.

\subsection{Derivation of an effective Hamiltonian}

What if the perturbation does not respect the time-reversal symmetry? We will now show that the simplest perturbation of this kind,

$$
V=-\sum_{j}\left(h_{x} \sigma_{j}^{x}+h_{y} \sigma_{j}^{y}+h_{z} \sigma_{j}^{z}\right)
$$

does open a spectral gap. (Physically, the vector $\mathbf{h}=\left(h_{x}, h_{y}, h_{z}\right)$ is an external magnetic field acting on all spins.) For simplicity we will assume that $J_{x}=J_{y}=J_{z}=J$.

Let us use the perturbation theory to construct an effective Hamiltonian $H_{\text {eff }}$ acting on the vortex-free sector. One can easily see that $H_{\mathrm{eff}}^{(1)}=0$. Although the second-order term $H_{\mathrm{eff}}^{(2)}$ does not vanish, it preserves the time-reversal symmetry. Therefore we must consider the third-order term, which can be written as follows:

$$
H_{\mathrm{eff}}^{(3)}=\Pi_{0} V G_{0}^{\prime}\left(E_{0}\right) V G_{0}^{\prime}\left(E_{0}\right) V \Pi_{0},
$$

where $\Pi_{0}$ is the projector onto the vortex-free sector, and $G_{0}^{\prime}$ is the unperturbed Green function with the vortex-free sector excluded. In principle, the Green function can be computed for each gauge sector using the formula $G_{0}(E)=-i \int_{0}^{\infty} e^{i\left(E-H_{0}+i \delta\right) t} d t$ (where $\delta$ is an infinitely small number). For fixed values of the field variables $u_{j k}$ the unperturbed Hamiltonian may be represented in the form (18) and exponentiated implicitly by exponentiating the corresponding matrix $A$; the final result may be written as a normal-ordered expansion up to the second order. However, it is a rather difficult calculation, so we will use a qualitative argument instead.

Let us assume that all intermediate states involved in the calculation have energy $\Delta E \sim|J|$ above the ground state. (Actually, $\Delta E \approx 0.27|J|$ for the lowest energy state with two adjacent vortices, see Appendix A] Then $G_{0}^{\prime}\left(E_{0}\right)$ can be replaced by $-\left(1-\Pi_{0}\right) /|J|$. The effective Hamiltonian becomes

$$
H_{\mathrm{eff}}^{(3)} \sim-\frac{h_{x} h_{y} h_{z}}{J^{2}} \sum_{j, k, l} \sigma_{j}^{x} \sigma_{k}^{y} \sigma_{l}^{z}
$$


where the summation takes place over spin triples arranged as follows:

a)

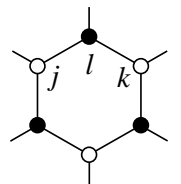

(and symmetry-equivalent), or

b)

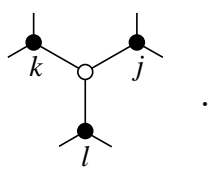

Configuration (a) corresponds to the term $\sigma_{j}^{x} \sigma_{k}^{y} \sigma_{l}^{z}=-i D_{l} \hat{u}_{j l} \hat{u}_{k l} c_{j} c_{k}$ (where $D_{l}$ may be omitted as we work in the physical subspace), or simply $-i c_{j} c_{k}$ in the standard gauge. Configuration (b) corresponds to a four-fermion term and therefore does not directly influence the spectrum. Thus we arrive at this effective Hamiltonian:

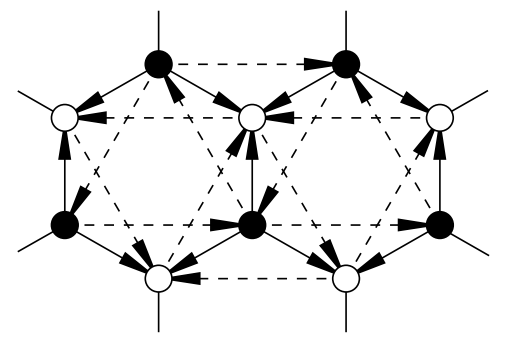

$$
\begin{aligned}
& H_{\mathrm{eff}}=\frac{i}{4} \sum_{j, k} A_{j k} c_{j} c_{k}, \\
& A=2 J(-)+2 \kappa(---), \\
& \kappa \sim \frac{h_{x} h_{y} h_{z}}{J^{2}} .
\end{aligned}
$$

Here $(-)$ is just another notation for $u^{\text {std }}$, i.e., the matrix whose entry $(-)_{j k}$ is equal to 1 if there is a solid arrow from $k$ to $j$ in the figure, -1 if an arrow goes from $j$ to $k$, and 0 otherwise. ( --- ) is defined similarly.

\subsection{The spectrum and the Chern number}

The fermionic spectrum $\varepsilon(\mathbf{q})$ of the Hamiltonian (48) is given by the eigenvalues of a modified matrix $i \widetilde{A}(\mathbf{q})$ (cf. Eq. (32) ) :

$$
i \widetilde{A}(\mathbf{q})=\left(\begin{array}{cc}
\Delta(\mathbf{q}) & i f(\mathbf{q}) \\
-i f(\mathbf{q})^{*} & -\Delta(\mathbf{q})
\end{array}\right), \quad \varepsilon(\mathbf{q})= \pm \sqrt{|f(\mathbf{q})|^{2}+\Delta(\mathbf{q})^{2}}
$$

where $f(\mathbf{q})=2 J\left(e^{i\left(\mathbf{q}, \mathbf{n}_{1}\right)}+e^{i\left(\mathbf{q}, \mathbf{n}_{2}\right)}+1\right)$ and $\Delta(\mathbf{q})=4 \kappa\left(\sin \left(\mathbf{q}, \mathbf{n}_{1}\right)+\sin \left(\mathbf{q},-\mathbf{n}_{2}\right)+\sin \left(\mathbf{q}, \mathbf{n}_{2}-\mathbf{n}_{1}\right)\right)$. Actually, the exact form of the function $\Delta(\mathbf{q})$ does not matter; the important parameter is

$$
\Delta=\Delta\left(\mathbf{q}_{*}\right)=-\Delta\left(-\mathbf{q}_{*}\right)=6 \sqrt{3} \kappa \sim \frac{h_{x} h_{y} h_{z}}{J^{2}}
$$

which determines the energy gap. The conic singularities are resolved as follows:

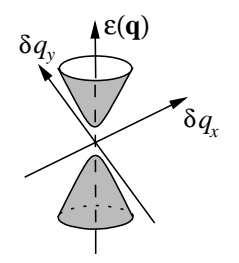

$$
\varepsilon(\mathbf{q}) \approx \pm \sqrt{3 J^{2}|\delta \mathbf{q}|^{2}+\Delta^{2}}
$$

where $\delta \mathbf{q}=\mathbf{q}-\mathbf{q}_{*}$ or $\delta \mathbf{q}=\mathbf{q}+\mathbf{q}_{*}$.

Remark 6.1. The magnetic field also gives nontrivial dispersion to vortices. Indeed, the operators $W_{p}$ are no longer conserved, therefore a vortex can hop to an adjacent hexagon. Thus the vortex energy depends on the momentum. This effect is linear in $h$, but it is not so important as the change in the fermionic spectrum. 
Let us also find the fermionic spectral projector, which determines the ground state. The global spectral projector $P$ is defined by Eq. (27); we now consider its Fourier component:

$$
\begin{aligned}
& \widetilde{P}(\mathbf{q})=\frac{1}{2}\left(1-\operatorname{sgn}(i \widetilde{A}(\mathbf{q}))=\frac{1}{2}\left(1+m_{x}(\mathbf{q}) \sigma^{x}+m_{y}(\mathbf{q}) \sigma^{y}+m_{z}(\mathbf{q}) \sigma^{z}\right),\right. \\
& \mathbf{m}(\mathbf{q}) \approx \begin{cases}\frac{1}{\sqrt{\left(\delta q_{x}\right)^{2}+\left(\delta q_{y}\right)^{2}+\Delta^{2} /\left(3 J^{2}\right)}}\left(-\delta q_{y},-\delta q_{x},-\frac{\Delta}{\sqrt{3} J}\right) & \text { if } \mathbf{q} \approx \mathbf{q}_{*}, \\
\frac{1}{\sqrt{\left(\delta q_{x}\right)^{2}+\left(\delta q_{y}\right)^{2}+\Delta^{2} /\left(3 J^{2}\right)}}\left(-\delta q_{y}, \delta q_{x}, \frac{\Delta}{\sqrt{3} J}\right) & \text { if } \mathbf{q} \approx-\mathbf{q}_{*} .\end{cases}
\end{aligned}
$$

The function $\mathbf{m}$ maps the torus to the unit sphere. If $\Delta>0$, then this map has degree 1 . Indeed, the neighborhood of $\mathbf{q}_{*}$ is mapped onto the lower hemisphere, the neighborhood of $-\mathbf{q}_{*}$ is mapped onto the upper hemisphere; in both cases the orientation is preserved. (The rest of the torus is mapped onto the equator.) For negative $\Delta$ the map has degree -1 .

An important topological quantity characterizing a two-dimensional system of noninteracting (or weakly interacting) fermions with an energy gap is the spectral Chern number. It plays a central role in the theory of the integer quantum Hall effect [43, 44, 1]. In our model there is no analogue of Hall conductivity (because the number of fermions is not conserved), but the Chern number determines the edge mode chirality and anyonic properties of vortices (cf. 48]).

The spectral Chern number is defined as follows. For each value of the momentum q we consider the space $\widetilde{L}(\mathbf{q})$ of annihilation operators, i.e., fermionic modes with negative energy; this is the subspace the matrix $\widetilde{P}(\mathbf{q})$ projects onto. Thus we obtain a complex vector bundle over the momentum space. (In our case $\widetilde{L}(\mathbf{q})$ is a one-dimensional subspace of $\mathbb{C}^{2}$, so the bundle is one-dimensional.) The first Chern number of this bundle is denoted by $\nu$ and can be expressed as follows (cf. [44]):

$$
\nu=\frac{1}{2 \pi i} \int \operatorname{Tr}(\widetilde{P} d \widetilde{P} \wedge d \widetilde{P})=\frac{1}{2 \pi i} \int \operatorname{Tr}\left(\widetilde{P}\left(\frac{\partial \widetilde{P}}{\partial q_{x}} \frac{\partial \widetilde{P}}{\partial q_{y}}-\frac{\partial \widetilde{P}}{\partial q_{y}} \frac{\partial \widetilde{P}}{\partial q_{x}}\right)\right) d q_{x} d q_{y} .
$$

The Chern number is always an integer. If the spectral projector $\widetilde{P}(\mathbf{q})$ is given by Eqs. (152), (53), then

$$
\nu=\frac{1}{4 \pi} \int\left(\frac{\partial \mathbf{m}}{\partial q_{x}} \times \frac{\partial \mathbf{m}}{\partial q_{y}}, \mathbf{m}\right) d q_{x} d q_{y}=\operatorname{sgn} \Delta= \pm 1
$$

We will use the notation $B_{\nu}$ (where $\nu= \pm 1$ ) to designate phase $B$ in the magnetic field. In the Abelian phases $A_{x}, A_{y}, A_{z}$ the Chern number is zero.

\section{Edge modes and thermal transport}

Remarkably, any system with nonzero Chern number possesses gapless edge modes. Such modes were first discovered in the integer quantum Hall effect [51]; they are chiral, i.e., propagate only in one direction (see Fig. 8). In fact, left-moving and right-moving modes may coexist, but the following relation holds [52]:

$$
\nu_{\text {edge }} \stackrel{\text { def }}{=}(\# \text { of left-movers }- \text { \# of right-movers })=\nu \text {. }
$$




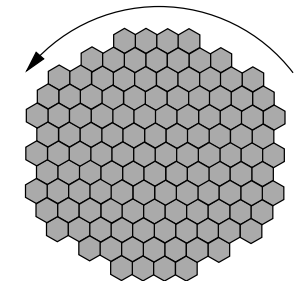

a)

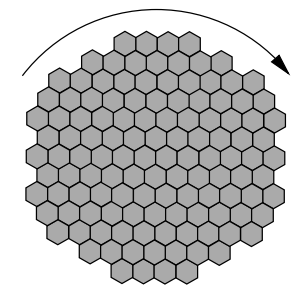

b)

Figure 8: Chiral edge modes: left-moving (a) and right-moving (b).

In the absence of special symmetry, counterpropagating modes usually cancel each other, so the surviving modes have the same chirality. A calculation of the edge spectrum in phases $B_{\nu}$ (for some specific boundary conditions) and a simple proof of Eq. (56) are given in Appendix B. More rigorous and general results, which even apply to disordered systems, can be found in Refs. [53, 54].

It is important to note that the analogy to the quantum Hall effect is not exact. In our model (like in two-dimensional superfluid and superconducting systems [55, 48]) edge modes are described by real fermions, as contrasted to complex fermions in the quantum Hall effect. Therefore each quantum Hall mode is equivalent to two modes in our system.

Chiral edge modes can carry energy, leading to potentially measurable thermal transport. (The temperature $T$ is assumed to be much smaller than the energy gap in the bulk, so that the effect of bulk excitations is negligible.) For quantum Hall systems, this phenomenon was discussed in Refs. [56, 57]. The energy current along the edge in the left (counterclockwise) direction is given by the following formula:

$$
I=\frac{\pi}{12} c_{-} T^{2}
$$

where $c_{-}$is some real number. (The factor $\pi / 12$ is introduced to make a connection to conformal field theory, see below.) It is remarkable that $c_{-}$does not depend on particular conditions at the edge, but rather on the bulk state. Indeed, the energy current is conserved, therefore it remains constant even if some conditions change along the edge. The effect is invariant with respect to time rescaling. Since the energy current has dimension (time) ${ }^{-2}$, it must be proportional to $T^{2}$. But the value of the dimensionless proportionality coefficient cannot be found using such simple arguments.

There are two standard ways to calculate the coefficient $c_{-}$. They both rely on certain assumptions but can be applied to our model, yielding this result:

$$
c_{-}=\frac{\nu}{2}
$$

The first argument [58] (adapted to real fermions) assumes translational invariance and the absence of interaction. Each edge mode is described by a free fermion with an energy spectrum $\varepsilon(q)$ such that $\varepsilon(-q)=-\varepsilon(q)$ and $\varepsilon(q) \rightarrow \pm \infty$ as $q \rightarrow \pm \infty$. The signs in the last two expressions agree if the mode propagates in the direction of positive $q$ (for simplicity we may assume that $\varepsilon(q)>0$ when $q$ is positive and $\varepsilon(q)<0$ when $q$ is negative). Thus the Hamiltonian has the form

$$
H=\frac{1}{2} \sum_{q} \varepsilon(q) a_{-q} a_{q}=\sum_{q: \varepsilon(q)>0} \varepsilon(q) a_{-q} a_{q} .
$$


If $\varepsilon(q)>0$, then $a_{q}$ is an annihilation operator and $a_{-q}=a_{q}^{\dagger}$ is the corresponding creation operator. The mode propagates with group velocity $v(q)=d \varepsilon / d q$, and the occupation number $n(q)$ is given by the Fermi distribution. The energy flow due to each mode propagating in the positive direction can be calculated as follows:

$$
I_{1}=\int_{\varepsilon(q)>0} n(q) \varepsilon(q) v(q) \frac{d q}{2 \pi}=\int_{\varepsilon(q)>0} \frac{\varepsilon(q)}{1+e^{\varepsilon(q) / T}} \frac{d \varepsilon}{d q} \frac{d q}{2 \pi}=\frac{1}{2 \pi} \int_{0}^{\infty} \frac{\varepsilon d \varepsilon}{1+e^{\varepsilon / T}}=\frac{\pi}{24} T^{2} .
$$

Each mode propagating in the opposite direction contributes $-I_{1}$, therefore $I=\frac{\pi}{24} \nu T^{2}$.

The second derivation [57] is based on the assumption that the edge modes can be described by a conformal field theory (CFT). In this case,

$$
c_{-}=c-\bar{c}
$$

where $c$ and $\bar{c}$ are the Virasoro central charges. Thus, $c_{-}$is called the chiral central charge. Leftmoving fermions have $(c, \bar{c})=\left(\frac{1}{2}, 0\right)$ whereas right-moving fermions have $(c, \bar{c})=\left(0, \frac{1}{2}\right)$, which implies Eq. (58). More generally, $c$ and $\bar{c}$ are some rational numbers, and so is $c_{-}$. The chiral central charge parametrizes a two-dimensional gravitational anomaly [59] of the corresponding CFT; it can also be identified with the coefficient in front of the gravitational Chern-Simons action in a three-dimensional theory [5]. Volovik [60] suggested that for ${ }^{3} \mathrm{He}-\mathrm{A}$ films, the role of gravitational field is played by the order parameter interacting with fermions. However, it is not obvious how to define a "gravitational field" for lattice models.

It remains a bit mysterious how the chiral central charge is related to the ground state and spin correlators in the bulk. This question is partially answered in Appendix D but the obtained expression for $c_{-}$is not easy to use, nor can we demonstrate that $c_{-}$is rational. Note that there is a beautiful relation between the chiral central charge and algebraic properties of anyons 17, 61, which does imply the rationality of $c_{-}$. We discuss that relation in Appendix $\mathbb{E}$ (see Eq. (172) on page 66), though it is unclear how to deduce it only considering the bulk. The only known argument is to assume that edge modes are described by a CFT, then one can use modular invariance 62. In fact, the modular invariance alone would suffice. In Appendix D we try to derive it from general principles, but again, encounter a problem.

\section{Non-Abelian anyons}

We continue the study of phase $B$ in the magnetic field. Now that all bulk excitations are gapped, their braiding rules must be well-defined. Of course, this is only true if the particles are separated by distances that are much larger than the correlation length associated with the spectrum (51). The correlation length may be defined as follows: $\xi=|\operatorname{Im} \mathbf{q}|^{-1}$, where $\mathbf{q}$ is a complex solution to the equation $\varepsilon(\mathbf{q})=0$. Thus

$$
\xi=\left|\frac{\sqrt{3} J}{\Delta}\right| \sim\left|\frac{J^{3}}{h_{x} h_{y} h_{z}}\right| .
$$

The braiding rules for vortices depend on the spectral Chern number $\nu$. Although $\nu$ is actually equal to +1 or -1 (depending on the direction of the magnetic field), one may formally consider a model with an arbitrary gapped fermionic spectrum, in which case $\nu$ may take any 


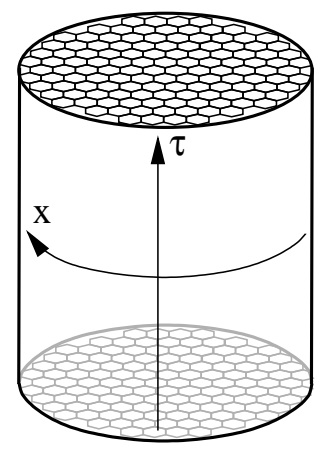

a)

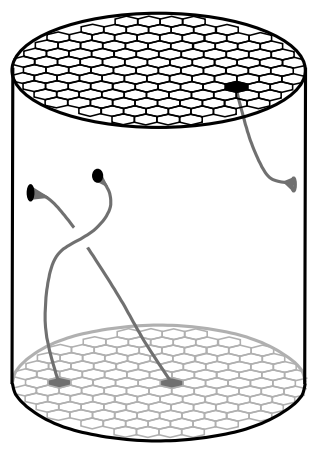

b)

Figure 9: The space-time coordinates (a) and particle worldlines (b).

integer value. We will see that vortices behave as non-Abelian anyons for any odd value of $\nu$, but their exact statistics depends on $\nu \bmod 16$.

The properties of the anyons are summarized in Table 1 on page 30. The notation and underlying concepts are explained below; see also Appendix E. Let us first show a quick way of deriving those properties from conformal filed theory (CFT) in the most important case, $\nu= \pm 1$. (For a general reference on CFT, see [63.) Then we will give an alternative derivation, which uses only rudimentary CFT and refers to the operational meaning of braiding and fusion.

\subsection{Bulk-edge correspondence}

The properties listed in Table 1 form the same type of algebraic structure that was described by Moore and Siberg [4] in the CFT context. However, the actual connection to CFT is indirect: anyons are related to edge modes, which in turn can be described by a field theory in $1+1$ dimensions. More concretely, the space-time may be represented as a cylinder (see Fig. 9). It is convenient to use the imaginary time formalism $(t=-i \tau$, where $\tau \in \mathbb{R})$, so that we have a two-dimensional Euclidean field theory on the side surface of the cylinder. The surface may be parametrized by a complex variable $z=\tau+i x$, where $x$ is the spatial coordinate.

The two-dimensional field theory describes the physics of the edge, which is generally richer than that of the bulk. The theory possesses both local and nonlocal fields. The insertion of a nonlocal field $\phi(\tau+i x)$ corresponds to an anyonic particle emerging on the edge or sinking into the bulk at point $(\tau, x)$. The correlation function of several nonlocal fields has nontrivial monodromy which coincides with the anyonic braiding. Specifically, the counterclockwise exchange of anyons in the bulk is equivalent to moving the fields counterclockwise on surface (if we look at the cylinder from outside). Moreover, the value of the correlator is not a number, but rather an operator transforming the initial anyonic state (on the bottom of the cylinder) into the final one (on the top of the cylinder). For non-Abelian anyons, the space of such operators is multidimensional.

The anyon-CFT correspondence has been successfully used in the study of quantum Hall systems [14, 15, 31]. The correspondence is well-understood if all boundary fields are either holomorphic or antiholomorphic, which is the case for our model. We have seen that the edge carries a left-moving (holomorphic) fermion for $\nu=1$, or a right-moving (antiholomorphic) 
Superselection sectors: 1 (vacuum), $\quad \varepsilon$ (fermion), $\sigma$ (vortex).

Quantum dimension: $\quad d_{1}=1, \quad d_{\varepsilon}=1 \quad d_{\sigma}=\sqrt{2}$;

Topological spin: $\quad \theta_{1}=1, \quad \theta_{\varepsilon}=-1, \quad \theta_{\sigma}=\theta=\exp \left(\frac{\pi}{8} i \nu\right)$;

Frobenius-Schur indicator: $\quad \varkappa_{1}=1, \quad \varkappa_{\varepsilon}=1, \quad \varkappa_{\sigma}=\varkappa=(-1)^{\left(\nu^{2}-1\right) / 8}$.

Global dimension: $\quad \mathcal{D}^{2} \stackrel{\text { def }}{=} \sum_{u} d_{u}^{2}=4$

Fusion rules: $\quad \varepsilon \times \varepsilon=1, \quad \varepsilon \times \sigma=\sigma, \quad \sigma \times \sigma=1+\varepsilon$.

Associativity relations:

$\bigvee_{1}^{\sigma}=\frac{\varkappa}{\sqrt{2}} \bigvee_{\sigma}^{\sigma}+\frac{\varkappa}{\sqrt{2}} \bigvee_{\varepsilon}^{\sigma}, \quad \bigvee_{\sigma}^{\sigma} \bigvee_{\sigma}^{\sigma}=\frac{\varkappa}{\sqrt{2}} \bigvee_{\sigma}^{\sigma}-\frac{\varkappa}{\sqrt{2}} \bigvee_{\sigma}^{\sigma}$,

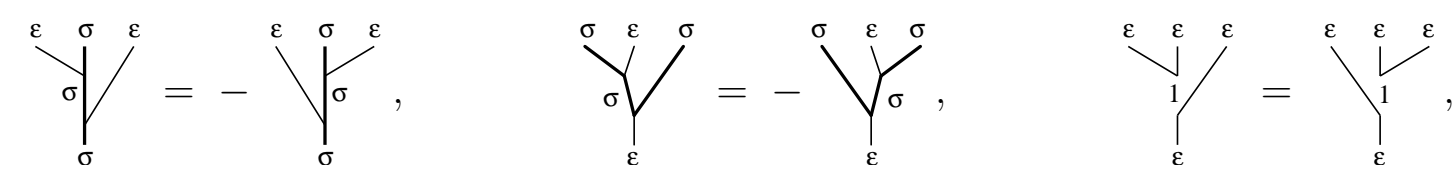

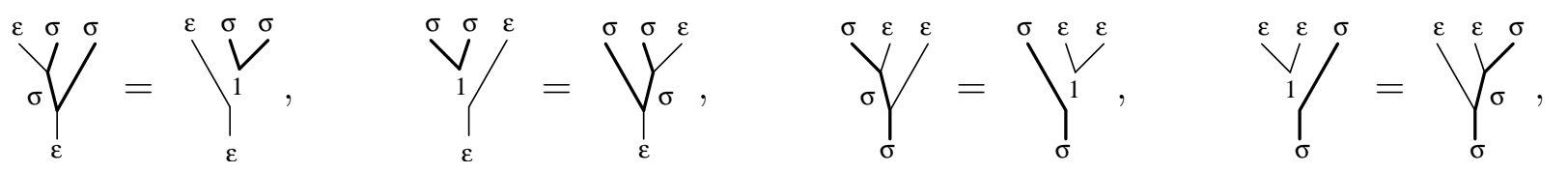

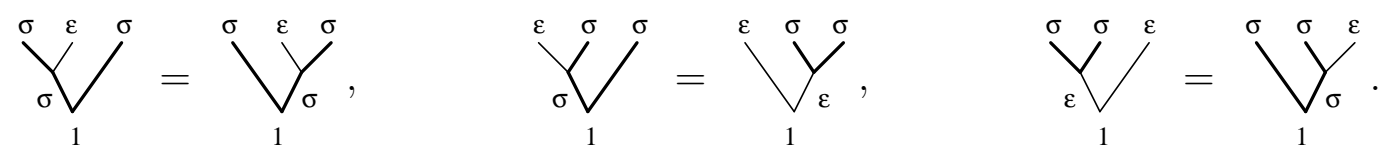

\section{Braiding rules:}

Definition of $R_{z}^{x y}$ :

$y_{\mathrm{z}}^{\mathrm{y}}=R_{z}^{x y} \bigcup_{\mathrm{z}}^{\mathrm{y}} ;$

$R_{1}^{\varepsilon \varepsilon}=-1$

$R_{1}^{\sigma \sigma}=\varkappa \exp \left(-\frac{\pi}{8} i \nu\right)$

$R_{\sigma}^{\varepsilon \sigma}=R_{\sigma}^{\sigma \varepsilon}=-i^{\nu}$

$R_{\varepsilon}^{\sigma \sigma}=\varkappa \exp \left(\frac{3 \pi}{8} i \nu\right)$.

\section{Topological $S$-matrix:}
$\left(S_{z}\right)_{x y} \stackrel{\text { def }}{=} \frac{1}{\mathcal{D}}$
$S_{1}=\left(\begin{array}{ccc}\frac{1}{2} & \frac{1}{2} & \frac{1}{\sqrt{2}} \\ \frac{1}{2} & \frac{1}{2} & -\frac{1}{\sqrt{2}} \\ \frac{1}{\sqrt{2}} & -\frac{1}{\sqrt{2}} & 0\end{array}\right)$
$\left(S_{\varepsilon}\right)_{\sigma \sigma}=\exp \left(-\frac{\pi}{4} i \nu\right)$

Table 1: Algebraic properties of anyons in non-Abelian phases ( $\nu$ is odd). 
fermion for $\nu=-1$. A vortex emerging on the surface corresponds to a twist field $\sigma$. The correlation functions for such fields are given by holomorphic (resp. antiholomorphic) conformal blocks for the Ising model.

A partial bulk-edge correspondence can be established at a more elementary level. Let $\nu=1$. A particularly important parameter of the edge theory is the conformal weight of the twist field, $\left(h_{\sigma}, \bar{h}_{\sigma}\right)=\left(\frac{1}{16}, 0\right)$. The related bulk parameter is the topological spin of a vortex, $\theta_{\sigma}$; it corresponds to the counterclockwise rotation by angle $2 \pi$. Since the rotations in the bulk and on the surface must agree, the following equation holds for an arbitrary field $a$ :

$$
\theta_{a}=e^{2 \pi i\left(h_{a}-\bar{h}_{a}\right)}
$$

Thus $\theta_{\sigma}=e^{i \pi / 8}$. Similarly, if $\nu=-1$, then $\theta_{\sigma}=e^{-i \pi / 8}$. In the general case, there are $|\nu|=2 n+1$ species of the free fermion; the twist field also comes with some multiplicity. ${ }^{3}$ One may argue that the conformal weight of the twist field is proportional to the number of fermionic species. Hence

$$
\theta_{\sigma}=e^{i \pi \nu / 8}
$$

\subsection{Unpaired Majorana modes}

We now begin a rather lengthy derivation of the properties listed in Table 1, dealing only with anyons in the bulk. First, we give a crude description of vortices by Majorana operators and find the fusion rules. The braiding rules and so-called associativity relations (also known as crossing symmetry, or $6 j$-symbols) are determined up to several free parameters; we discuss what combinations of these parameters have invariant meaning. Solving the so-called pentagon and hexagon equations, we reduce the continuum of possibilities down to eight consistent theories. The right theory is selected using Eq. (62).

The starting point is this: if $\nu$ is odd, then each vortex carries an unpaired Majorana mode. A sufficiently rigorous proof of this statement can be found in Appendix C. Here we give a rough explanation based on the quantum Hall analogy.

It is known that the quantized Hall conductivity for noninteracting electrons (in units of $\left.e^{2} / h\right)$ is equal to the Chern number of the projector onto the occupied electron states [44]. The essential difference from our case is that electrons are ordinary fermions, not Majorana fermions. However, we can concoct an analogue of an electron system from two copies of the Majorana system, which may be pictured as two layers. The Hamiltonian is as follows:

$$
H_{\text {electron }}=\frac{i}{4} \sum_{j, k} A_{j k}\left(c_{j}^{\prime} c_{k}^{\prime}+c_{j}^{\prime \prime} c_{k}^{\prime \prime}\right)=\sum_{j, k} i A_{j k} a_{j}^{\dagger} a_{k}, \quad \text { where } a_{j}=\frac{1}{2}\left(c_{j}^{\prime}+i c_{j}^{\prime \prime}\right) .
$$

(We forget about the original spin model for the purpose of this construction.) Note that the Hamiltonian possesses a global $\mathrm{O}(2)$ symmetry that consists of orthogonal linear transformations $c_{j}^{\prime} \mapsto \alpha c_{j}^{\prime}+\beta c_{j}^{\prime \prime}, c_{j}^{\prime \prime} \mapsto \gamma c_{j}^{\prime}+\delta c_{j}^{\prime \prime}$. The rotational subgroup $\mathrm{U}(1) \cong \mathrm{SO}(2) \subset \mathrm{O}(2)$ corresponds to the conservation of electric charge, whereas reflections (i.e., transformations with determinant -1 ) change the sign of the charge.

\footnotetext{
${ }^{3}$ Specifically, there are $2^{n}$ copies of the twist field, which are transformed according to the fundamental representation of $\operatorname{Spin}(2 n+1)$.
} 
Assuming that the Fermi energy is zero, the projector onto the occupied electron states coincides with the spectral projector for the Majorana system. Thus the Hall conductivity of the electron system is $\nu$.

A vortex piercing both Majorana layers corresponds to half-integer magnetic flux. Such a vortex carries excessive charge $q=\nu / 2+n$, where $n$ is an arbitrary integer. In particular, if $\nu$ is odd, a state with $q=1 / 2$ exist. By an $O(2)$ reflection, it is related to a $q=-1 / 2$ state with the same energy. The doublet of states with unit charge difference may be attributed to a zero-energy electron mode that can be empty or occupied. This mode can be represented by two Majorana modes. Since the layers are independent, we conclude that each layer contains one zero-energy Majorana mode.

It has been previously shown that zero-energy Majorana modes exist in some exotic onedimensional systems [64] as well as vortices in two-dimensional $p$-wave superconductors [48, 65]. A pair of Majorana modes at two vortices constitutes a full fermionic mode with a twodimensional Fock space. The quantum state of such a pair is virtually inaccessible to measurements or perturbations as long as the vortices stay far apart from each other. A system of $2 n$ vortices possesses a protected space of dimensionality $2^{n}$ (or $2^{n-1}$, if we require that the whole system have even fermionic parity).

\subsection{Fusion and braiding rules}

In our model a vortex carrying an unpaired Majorana mode is just one of the superselection sectors, denoted by $\sigma$. The other sectors are 1 (the vacuum) and $\varepsilon$ (a fermion). If two vortices fuse, they either annihilate completely or leave a fermion behind: $\sigma \times \sigma=1+\varepsilon$. The actual fusion outcome depends on the initial quantum state. Hence the protected space of the vortex pair has two basis vectors: $\left|\psi_{1}^{\sigma \sigma}\right\rangle$ and $\left|\psi_{\varepsilon}^{\sigma \sigma}\right\rangle$. (The upper indices indicate the particle types before the fusion whereas the subscript indicates the resulting particle.) The complete set of fusion rules is as follows:

$$
\varepsilon \times \varepsilon=1, \quad \varepsilon \times \sigma=\sigma, \quad \sigma \times \sigma=1+\varepsilon,
$$

plus trivial rules of the form $1 \times x=x$. Read backwards, these relations are understood as splitting rules: for example, an $\varepsilon$-particle can split into two $\sigma$-particles. However, these rules do not capture more subtle aspects of fusion and splitting, which will be discussed later.

Braiding rules for Majorana half-vortices in a spin-triplet superconductor have been derived by D. Ivanov 65. Here we follow the main idea of Ivanov's work. We should, however, keep in mind one important difference between his setting and our model. A spin-triplet superconductor has a locally measurable vector order parameter, which contributes to vortex-vortex interaction and can interact with impurities. One vortex making a full turn around another may pick up a nonuniversal phase, hence the non-Abelian statistics is defined up to arbitrary phase factors. That is not the case for our model (or for spinless superconductors [48]), so additional arguments are required to find the Abelian part of the vortex statistics.

Once again, we use the fact that each vortex $p$ carries an unpaired Majorana mode $C_{p}$, which is a linear combination of the operators $c_{j}$ on neighboring sites. The operators $c_{j}$ do not commute with gauge transformations and therefore should be used with care. The gauge can be fixed in a neighborhood of each vortex, so constructing the linear combination is not a problem. However, the overall sign of $C_{p}$ does not have invariant meaning. This ambiguity 
is avoided if we consider fermionic path operators (16), which are gauge-invariant. A suitable linear combination of elementary paths constitutes a path that begins or ends at a vortex.

Let us choose some reference path $l_{p}$ connecting each vortex $p=1,2 \ldots$ to a reference point 0 . We will assume that the vortices lie on the horizontal axis whereas the reference point 0 is located in the lower half-plane. Fixing the gauge along the paths, we may write

$$
W\left(l_{p}\right)=C_{p} c_{0} .
$$

Let us exchange vortices 1 and 2 by moving them counterclockwise. The exchange process is described by a unitary operator $R$ acting on the physical Hilbert space. It also acts on operators by conjugation: $X \mapsto R X R^{\dagger}$. Clearly,
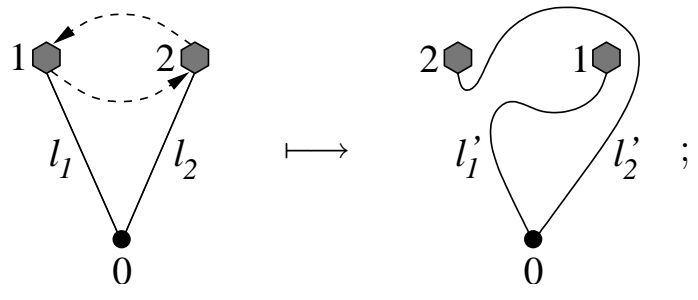

$$
\begin{aligned}
& R W\left(l_{1}\right) R^{\dagger}=W\left(l_{1}^{\prime}\right)=W\left(l_{2}\right), \\
& R W\left(l_{2}\right) R^{\dagger}=W\left(l_{2}^{\prime}\right)=-W\left(l_{1}\right) .
\end{aligned}
$$

(We have used the fact that $W\left(l_{2}^{\prime}\right)=-W\left(l_{1}\right)$. Indeed, the paths $l_{2}^{\prime}$ and $l_{1}$ differ by a loop enclosing a vortex; transporting a fermion around the vortex gives rise to the minus sign.) Hence

$$
\begin{aligned}
& R C_{1} R^{\dagger}=C_{2}, \\
& R C_{2} R^{\dagger}=-C_{1},
\end{aligned} \quad R=\theta \exp \left(-\frac{\pi}{4} C_{1} C_{2}\right),
$$

where $\theta$ is a phase factor. (We will see that $\theta$ is actually the topological spin of a vortex; for now it is just an unknown parameter).

At first sight, it is not clear whether the number $\theta$ has an invariant meaning. Indeed, the operator that moves a vortex along a given path may be defined up to an arbitrary phase. However, the ambiguity can be avoided by a careful definition of the particle exchange process. The following argument is completely general; it is not based on adiabaticity or translational invariance.

Let us consider a vortex path operator $W_{\sigma}(l)$ that is composed of elementary steps, i.e., displacements of the vortex from hexagon to hexagon. ${ }^{4}$ Each elementary displacement is defined up to a phase, but the path operator satisfies these equations:

$$
W_{\sigma}(\bar{l})=W_{\sigma}(l)^{\dagger}, \quad W_{\sigma}\left(l_{1} l_{2}\right)=W_{\sigma}\left(l_{2}\right) W_{\sigma}\left(l_{1}\right) .
$$

Here $\bar{l}$ denotes the reverse path, whereas $l_{1} l_{2}$ is the composite path (the vortex goes along $l_{1}$ and then along $l_{2}$ ). Remarkably, two vortices can be exchanged in such a way that the arbitrary phases cancel each other, see Fig. 10. The vortices move along the indicated lines; each line segment is passed by a vortex in both directions.

As mentioned above, the vortex pair has two states corresponding to the possible fusion outcomes, $\left|\psi_{1}^{\sigma \sigma}\right\rangle$ and $\left|\psi_{\varepsilon}^{\sigma \sigma}\right\rangle$. They should be identified with the eigenvectors of $C_{1} C_{2}$, but we do not know which is which. We can only write:

$$
C_{1} C_{2}\left|\psi_{1}^{\sigma \sigma}\right\rangle=i \alpha\left|\psi_{1}^{\sigma \sigma}\right\rangle, \quad C_{1} C_{2}\left|\psi_{\varepsilon}^{\sigma \sigma}\right\rangle=-i \alpha\left|\psi_{\varepsilon}^{\sigma \sigma}\right\rangle
$$

\footnotetext{
${ }^{4}$ The displacement may be realized as the action of $\sigma^{\alpha}$ on one of the two spins at the boundary between the hexagons (which changes the field configuration) followed by an operator of the form $\exp \left(\sum F_{k m} c_{k} c_{m}\right)$ (which adjusts the fermionic subsystem).
} 

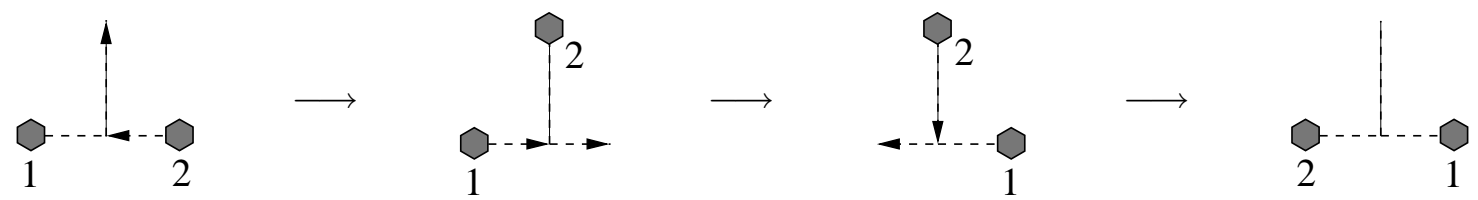

Figure 10: A realization of the particle exchange in which all local contributions cancel, revealing the topological effect in a pure form.

where $\alpha= \pm 1$ is unknown. Thus the braiding operator $R$ acts as follows:

$$
\begin{aligned}
R\left|\psi_{1}^{\sigma \sigma}\right\rangle & =R_{1}^{\sigma \sigma}\left|\psi_{1}^{\sigma \sigma}\right\rangle, & & R_{1}^{\sigma \sigma}=\theta e^{i \alpha \pi / 4}, \\
R\left|\psi_{\varepsilon}^{\sigma \sigma}\right\rangle & =R_{\varepsilon}^{\sigma \sigma}\left|\psi_{\varepsilon}^{\sigma \sigma}\right\rangle, & \text { where } & R_{\varepsilon}^{\sigma \sigma}=\theta e^{-i \alpha \pi / 4} .
\end{aligned}
$$

Remark 8.1. One may wonder why formula (67) contains the minus sign in front of $C_{1}$, but not in front of $C_{2}$. This is, in fact, a consequence of the convention that the reference point 0 lies in the lower half-plane. If we move it to the upper half-plane, the signs will change, $\alpha$ will turn into $-\alpha$, and the signs in front of $\alpha$ in Eq. (701) will change too. The numbers $R_{1}^{\sigma \sigma}, R_{\varepsilon}^{\sigma \sigma}$ and $\theta$ are invariant though.

\subsection{Associativity relations}

The fusion rules only indicate what fusion and splitting events are possible. Nontrivial relations arise if we consider a sequence of such events.

8.4.1 Relations based on the anticommutativity of fermionic operators. Let us consider the splitting of a $\sigma$-particle into $\varepsilon, \sigma$, and $\varepsilon$. This can be done in two different ways, depending on the order the two $\varepsilon$-particles are created. Let us suppose that the $\sigma$-particle is located at point 2 in the middle; the $\varepsilon$-particles will appear at points 1 and 3 on the left and on the right, respectively. The particles (or their future locations) are connected by the paths $l_{12}$ and $l_{23}$.

The $\sigma$-particle is described by the associated Majorana mode $C_{2}$. The $\varepsilon$-particles can be created from the vacuum by operators $a_{1}^{\dagger}$ and $a_{3}^{\dagger}$. However, these operators are not physical by themselves. To actually split the first $\varepsilon$-particle off the $\sigma$-particle, one needs to apply a fermionic path operator $W\left(l_{12}\right)$, which equals $a_{1}^{\dagger} C_{2}$ in a suitable gauge. The second $\varepsilon$-particle is created by the operator $W\left(l_{23}\right)=a_{3}^{\dagger} C_{2}$. Since $W\left(l_{12}\right) W\left(l_{23}\right)=-W\left(l_{23}\right) W\left(l_{12}\right)$, we obtain the following associativity relation between the two splitting processes:

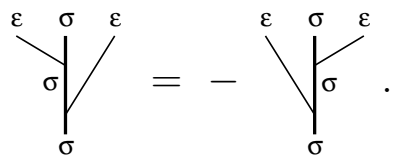

Similarly, we can consider the splitting of one $\varepsilon$-particle into three. For this, we use two conventions: $^{5}$ (i) moving an $\varepsilon$-particle from place $j$ to place $k$ is described by the operator $a_{k}^{\dagger} a_{j}$;

\footnotetext{
${ }^{5}$ Although these conventions are somewhat arbitrary, the result is invariant (see subsection 8.4.2).
} 
(ii) creating a fermionic pair from the vacuum is described by $a_{j}^{\dagger} a_{k}^{\dagger}$, where $j$ is located left of $k$. Thus we get:

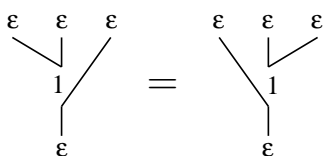

because $\left(a_{1}^{\dagger} a_{2}^{\dagger}\right)\left(a_{3}^{\dagger} a_{2}\right)=\left(a_{2}^{\dagger} a_{3}^{\dagger}\right)\left(a_{1}^{\dagger} a_{2}\right)$. (In these graphs the "up" direction is time. The intermediate state of the middle particle is shown in all cases, but lines corresponding to label 1, i.e., the vacuum superselection sector, are suppressed.)

8.4.2 Some generalities. The analysis of other splitting processes (e.g., $\sigma \rightarrow \sigma \sigma \sigma)$ is more complicated. Ideally, we would use the vortex path operator, but its exact form is unknown. Therefore we have to rely on more abstract arguments. Before we proceed, let us clarify some points pertaining to anyons in general.

1. Speaking about anyons, we are interested in particle types (i.e., superselection sectors) and topology of the particle worldlines (braids, trees, etc.). An actual particle is also characterized by position and local degrees of freedom; for example, an $\varepsilon$-particle may be "elementary" or consist of two adjacent vortices. We generally ignore such details though.

2. In the study of splitting and fusion, we consider particles located on the horizontal axis. In this case, we only care about the order of the particles on the line, but not about their positions. All configurations with the same particle types and order can be identified with each other. Unlike in the two-dimensional case, cycling through several configurations has trivial effect on the quantum state. ${ }^{6}$

3. "Quantum state of a particle" is a rather subtle notion. It may be understood as a projector that enforces certain spin correlations in some neighborhood of the supposed particle location. (The neighborhood radius must be much larger than the correlation length). Such an object has no "overall phase". Superpositions of states from different superselection sectors cannot be constructed either.

4. In spite of that, the state $\left|\psi_{1}^{\sigma \sigma}\right\rangle$ has a well-defined phase if we consider it relative to the vacuum. A similar argument holds for an arbitrary state $\left|\psi_{z}^{x y}\right\rangle$, which describes particles $x$ and $y$ with total "anyonic charge" $z$. For a general anyonic system, the $x y$-pair may have several distinct states that belongs to the same superselection sector $z$; in other words, there may be several ways to split $z$ into $x$ and $y$. Such states form a finite-dimensional Hilbert space $V_{z}^{x y}$, which is called fusion space. ${ }^{7}$ In our model, all such spaces have dimension one or zero. For example, $\left|\psi_{1}^{\sigma \sigma}\right\rangle$ is a unit vector in the one-dimensional space $V_{1}^{\sigma \sigma}$.

\footnotetext{
${ }^{6}$ This claim can be justified as follows. To move a particle $z$ from one place to another, we apply a path operator $W_{z}\left(r, r^{\prime}\right)$, which acts on the spins in some neighborhood of the interval $\left[r, r^{\prime}\right]$. These operators are defined in such a way that it does not matter whether the particle is moved at once or in several steps (cf. Eq. (68)). When several particles are present, we do not let them pass through each other, which imposes a certain restriction on the sequence of operators applied. All such sequences are actually equivalent since the path operators for nonoverlapping intervals commute.

${ }^{7}$ Technically, it should be called "splitting space", whereas the fusion space is its dual, $V_{x y}^{z}=\left(V_{z}^{x y}\right)^{*}$.
} 
For a more rigorous definition of the space $V_{z}^{x y}$, consider the splitting of $z$ into $x$ and $y$ by an operator $L$ that acts on the spins in some fixed finite region $\Omega$. (We call such operators local.) Let us also fix a quantum state $\left|\Psi_{z}\right\rangle$ that has a $z$-particle at a given place and no other particles in $\Omega$ or within the correlation length from $\Omega$. Finally, we consider the set of local operators $L$ for which the state $L\left|\Psi_{z}\right\rangle$ has an $x$-particle and an $y$-particle at the required places. By definition, these are all possible states of the $x y$-pair that can be obtained from $z$. On the other hand, such states are in one-to-one correspondence with equivalence classes of operators $L$ : we say that $L$ and $L^{\prime}$ are equivalent if $L\left|\Psi_{z}\right\rangle=L^{\prime}\left|\Psi_{z}\right\rangle$. For local operators, the equivalence relation does not depend on the choice of $\left|\Psi_{z}\right\rangle$. Thus we arrive at the following definition:

$V_{z}^{x y}$ is the set of equivalence classes of local operators that split $z$ into $x$ and $y$.

Each vertex in a splitting graph (as in Eqs. (71), (72) above) designates an equivalence class of local operators. For example, ${\underset{\sigma}{\sigma}}_{\sigma}^{\sigma}=\left|\psi_{\sigma}^{\varepsilon \sigma}\right\rangle \in V_{\sigma}^{\varepsilon \sigma}$. One may choose an arbitrary basis in the space $V_{z}^{x y}$. In our case, this amounts to fixing the phase of the vectors $\left|\psi_{1}^{\sigma \sigma}\right\rangle,\left|\psi_{\varepsilon}^{\sigma \sigma}\right\rangle,\left|\psi_{\sigma}^{\varepsilon \sigma}\right\rangle$, $\left|\psi_{\sigma}^{\sigma \varepsilon}\right\rangle$, and $\left|\psi_{1}^{\varepsilon \varepsilon}\right\rangle$. Different bases are related by a transformation of the form

$$
\left|\psi_{z}^{x y}\right\rangle^{\prime}=u_{z}^{x y}\left|\psi_{z}^{x y}\right\rangle, \quad\left|u_{z}^{x y}\right|=1
$$

which generally affects relations like (171) and (72). However, these particular relations are invariant since they have the same set of basis vectors on both sides: $\left|\psi_{\sigma}^{\varepsilon \sigma}\right\rangle,\left|\psi_{\sigma}^{\sigma \varepsilon}\right\rangle$ in Eq. (171) and $\left|\psi_{1}^{\varepsilon \varepsilon}\right\rangle$ in Eq. (72).

8.4.3 More relations. Let us write some other possible associativity relations in a general form:
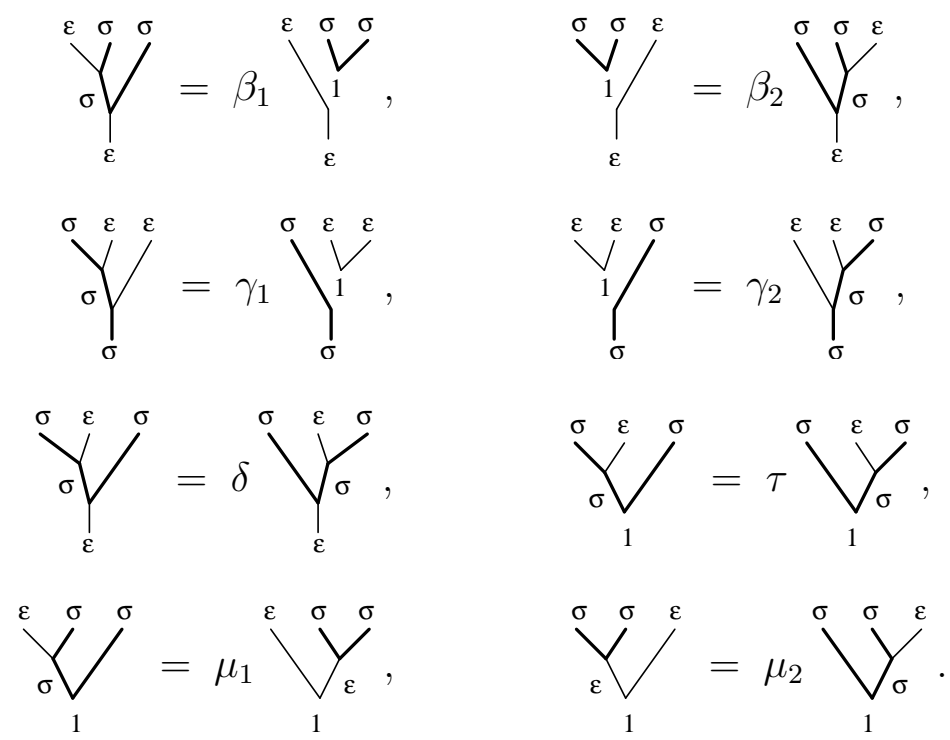

The numbers $\beta_{1}, \beta_{2}, \gamma_{1}, \gamma_{2}, \delta, \tau, \mu_{1}, \mu_{2}$ are equal to 1 in magnitude. These and similar coefficients are called structural parameters.

Using the transformation (173), we can eliminate some of the parameters, e.g., $\beta_{1}, \beta_{2}$, and $\gamma_{1}$. Indeed, they are transformed as follows:

$$
\beta_{1}^{\prime}=\frac{u_{\sigma}^{\varepsilon \sigma} u_{\varepsilon}^{\sigma \sigma}}{u_{1}^{\sigma \sigma}} \beta_{1}, \quad \beta_{2}^{\prime}=\frac{u_{1}^{\sigma \sigma}}{u_{\sigma}^{\sigma \varepsilon} u_{\varepsilon}^{\sigma \sigma}} \beta_{2}, \quad \gamma_{1}^{\prime}=\frac{\left(u_{\sigma}^{\sigma \varepsilon}\right)^{2}}{u_{1}^{\varepsilon \varepsilon}} \gamma_{1} .
$$


We require that $\beta_{1}^{\prime}=\beta_{2}^{\prime}=\gamma_{1}^{\prime}=1$ and solve for $u_{z}^{x y}$. Note that the solution is unique up to trivial variations not affecting the structural parameters, namely

$$
u_{z}^{x y} \mapsto \zeta_{x} \zeta_{y} \zeta_{z}^{-1} u_{z}^{x y}
$$

Finally, let us consider the splitting of one $\sigma$-particle into three $\sigma$-particles. The space $V_{\sigma}^{\sigma \sigma \sigma}$ is two-dimensional. A basis in this space can be chosen in two ways:

$$
\left\{\left|\xi_{1}\right\rangle=\bigvee_{1}^{\sigma} \gamma_{\sigma}^{\sigma}, \quad\left|\xi_{\varepsilon}\right\rangle=\bigvee_{\sigma}^{\sigma} Y_{\sigma}^{\sigma}\right\} \text { or } \quad\left\{\left|\eta_{1}\right\rangle=\bigvee_{\sigma}^{\sigma}, \quad\left|\eta_{\varepsilon}\right\rangle=\bigvee_{\sigma}^{\sigma}\right\} .
$$

Of course, one basis can be expressed in terms of the other:

$$
\left|\xi_{1}\right\rangle=a_{11}\left|\eta_{1}\right\rangle+a_{\varepsilon 1}\left|\eta_{\varepsilon}\right\rangle, \quad\left|\xi_{\varepsilon}\right\rangle=a_{1 \varepsilon}\left|\eta_{1}\right\rangle+a_{\varepsilon \varepsilon}\left|\eta_{\varepsilon}\right\rangle, \quad \text { where } \quad a_{x y}=\left\langle\eta_{x} \mid \xi_{y}\right\rangle .
$$

The coefficients $a_{x y}$ and the other structural parameters will be found later. At this point, we can only tell that the matrix $\left(a_{x y}\right)$ is unitary.

To illustrate the physical meaning of the structural parameters, let us return to the gedanken experiment considered in the introduction. Suppose we create two pairs of $\sigma$-particles from the vacuum. Then we take one particle from each pair and fuse them. With probability $\left|a_{11}\right|^{2}$ they will annihilate, and with probability $\left|a_{\varepsilon 1}\right|^{2}$ they will fuse into an $\varepsilon$-particle. (We will see that $\left|a_{11}\right|^{2}=\left|a_{\varepsilon 1}\right|^{2}=1 / 2$.)

\subsection{Algebraic consistency}

All the previous arguments were based on the result that vortices carry unpaired Majorana modes. Not surprisingly, this property alone is not sufficient to fully characterize the fusion and braiding of anyons. We now invoke some additional principles. The first one is consistency: successive fusion and braiding events must commute with each other in certain cases. A more careful statement of this requirement amounts to the formulation of an algebraic theory of anyons.

Anyons may be described in the framework of topological quantum field theory (TQFT), which originates from Witten's paper on quantum Chern-Simons fields [5] and the work of Moore and Siberg on conformal field theory 4. Important mathematical studies in this area were done by Reshetikhin and Turaev [66] and Walker [67]. For our purposes, it suffices to use a construction called unitary modular category (UMC), which constitutes the algebraic core of TQFT 68. This construction will be outlined in Appendix [E. Actually, the full theory is not necessary to understand the calculations below. On the contrary, one may use these calculations to motivate some of the UMC axioms.

One of the axioms is known as the pentagon equation, see Fig. 177 on page 69, It deals with the five ways to split a particle $u$ into $x, y, z, w$, or five representation of the space $V_{u}^{x y z w}$. The arrows in the figure may be regarded as equality signs, therefore the diagram must commute. For example, consider the splitting process $\sigma \rightarrow \varepsilon \sigma \sigma \sigma$ via intermediate states $q=1$ and $p=\sigma$. 
The upper path across the diagram looks like this:

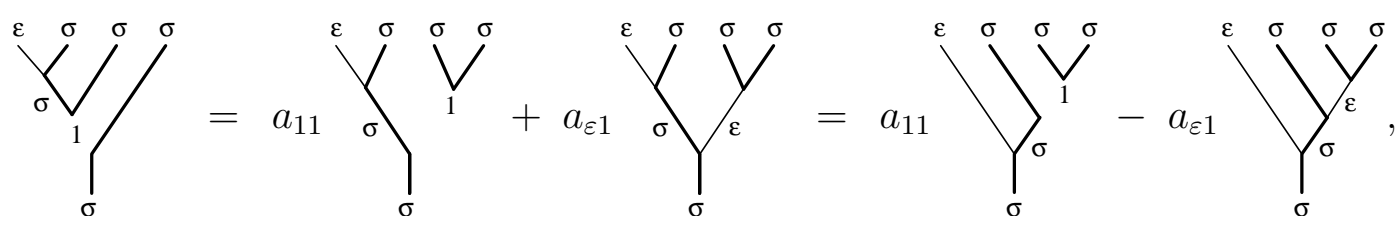

whereas the lower path is as follows:

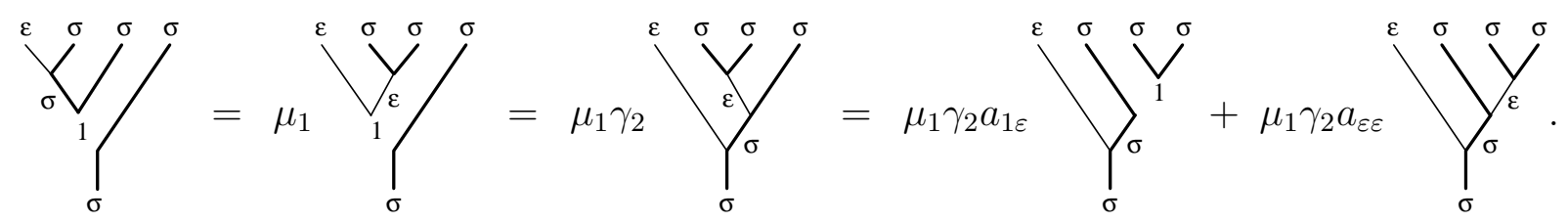

Thus $\mu_{1} \gamma_{2} a_{1 \varepsilon}=a_{11}, \mu_{1} \gamma_{2} a_{\varepsilon \varepsilon}=-a_{\varepsilon 1}$. One can also start with the splitting graph that has intermediate state $\varepsilon$ instead of 1 ; this gives another pair of relations between the structural parameters.

Overall, there are 23 spaces $V_{u}^{x y z w}$ with nontrivial upper indices. Thus we obtain 23 pentagon equations, 5 of which are satisfied automatically. The remaining 18 equations imply the following relations between the structural parameters:

$$
\begin{aligned}
& V_{\sigma}^{\varepsilon \sigma \sigma \sigma}:\left\{\begin{array}{rl}
\mu_{1} \gamma_{2} a_{1 \varepsilon} & =a_{11}, \\
\mu_{1} \gamma_{2} a_{\varepsilon \varepsilon} & =-a_{\varepsilon 1}, \\
\beta_{1} a_{11} & =a_{1 \varepsilon}, \\
\beta_{1} a_{\varepsilon 1} & =-a_{\varepsilon \varepsilon} ;
\end{array} \quad V_{\sigma}^{\sigma \sigma \sigma \varepsilon}:\left\{\begin{aligned}
\mu_{2} \gamma_{1} a_{\varepsilon 1} & =a_{11}, \\
\beta_{2} a_{11} & =a_{\varepsilon 1}, \\
\mu_{2} \gamma_{1} a_{\varepsilon \varepsilon} & =-a_{1 \varepsilon} \\
\beta_{2} a_{1 \varepsilon} & =-a_{\varepsilon \varepsilon}
\end{aligned}\right.\right. \\
& V_{\sigma}^{\sigma \varepsilon \sigma \sigma}:\left\{\begin{array}{l}
\tau \beta_{1} a_{\varepsilon 1}=a_{11}, \\
\tau \mu_{1} a_{11}=\gamma_{1} a_{\varepsilon 1}, \\
\delta \beta_{1} a_{\varepsilon \varepsilon}=a_{1 \varepsilon}, \\
\delta \mu_{1} a_{1 \varepsilon}=\gamma_{1} a_{\varepsilon \varepsilon} ;
\end{array} \quad V_{\sigma}^{\sigma \sigma \varepsilon \sigma}:\left\{\begin{array}{l}
\tau \mu_{2} a_{11}=\gamma_{2} a_{1 \varepsilon}, \\
\delta \mu_{2} a_{\varepsilon 1}=\gamma_{2} a_{\varepsilon \varepsilon}, \\
\tau \beta_{2} a_{1 \varepsilon}=a_{11}, \\
\delta \beta_{2} a_{\varepsilon \varepsilon}=a_{\varepsilon 1} ;
\end{array}\right.\right. \\
& V_{1}^{\sigma \sigma \sigma \sigma}:\left\{\begin{array}{r}
a_{11}^{2}+\tau a_{1 \varepsilon} a_{\varepsilon 1}=1 \\
a_{\varepsilon 1} a_{11}+\tau a_{\varepsilon \varepsilon} a_{\varepsilon 1}=0 \\
a_{11} a_{1 \varepsilon}+\tau a_{1 \varepsilon} a_{\varepsilon \varepsilon}=0 \\
a_{\varepsilon 1} a_{1 \varepsilon}+\tau a_{\varepsilon \varepsilon}^{2}=1
\end{array}\right. \\
& V_{\varepsilon}^{\sigma \sigma \sigma \sigma}:\left\{\begin{aligned}
a_{11}^{2}+\delta a_{1 \varepsilon} a_{\varepsilon 1} & =0 \\
a_{\varepsilon 1} a_{11}+\delta a_{\varepsilon \varepsilon} a_{\varepsilon 1} & =\beta_{2} \\
a_{11} a_{1 \varepsilon}+\delta a_{1 \varepsilon} a_{\varepsilon \varepsilon} & =\beta_{1} \\
a_{\varepsilon 1} a_{1 \varepsilon}+\delta a_{\varepsilon \varepsilon}^{2} & =0
\end{aligned}\right. \\
& V_{1}^{\sigma \varepsilon \varepsilon \sigma}: \quad \tau^{2}=\gamma_{1} \gamma_{2}, \\
& V_{1}^{\varepsilon \varepsilon \sigma \sigma}, V_{\varepsilon}^{\varepsilon \varepsilon \sigma \sigma}: \quad \mu_{1} \beta_{1} \gamma_{2}=1, \\
& V_{\varepsilon}^{\sigma \varepsilon \varepsilon \sigma}: \delta^{2}=\gamma_{1} \gamma_{2}, \\
& V_{1}^{\sigma \sigma \varepsilon \varepsilon}, V_{\varepsilon}^{\sigma \sigma \varepsilon \varepsilon}: \quad \mu_{2} \beta_{2} \gamma_{1}=1 \text {, } \\
& V_{1}^{\varepsilon \sigma \varepsilon \sigma}, V_{\varepsilon}^{\varepsilon \sigma \varepsilon \sigma}, V_{1}^{\sigma \varepsilon \sigma \varepsilon}, V_{\varepsilon}^{\sigma \varepsilon \sigma \varepsilon}: \quad \delta=-\tau, \\
& V_{1}^{\varepsilon \sigma \sigma \varepsilon}, V_{\varepsilon}^{\varepsilon \sigma \sigma \varepsilon}: \mu_{1} \mu_{2}=\beta_{1} \beta_{2} .
\end{aligned}
$$

Equations (82 85) have only two solutions satisfying the convention that $\beta_{1}=\beta_{2}=\gamma_{1}=1$ :

$$
\begin{aligned}
& \beta_{1}=\beta_{2}=\gamma_{1}=\gamma_{2}=\mu_{1}=\mu_{2}=\tau=1, \quad \delta=-1, \\
& \left(\begin{array}{ll}
a_{11} & a_{1 \varepsilon} \\
a_{\varepsilon 1} & a_{\varepsilon \varepsilon}
\end{array}\right)=\frac{\varkappa}{\sqrt{2}}\left(\begin{array}{cc}
1 & 1 \\
1 & -1
\end{array}\right), \quad \text { where } \varkappa= \pm 1 .
\end{aligned}
$$


Remark 8.2. The number of independent equations can be reduced by using a symmetry between upper and lower indices: $V_{z}^{x y} \cong V_{\bar{y}}^{\bar{z} x}$, where $\bar{z}$ denotes the antiparticle for $z$ (in our case, $\bar{\varepsilon}=\varepsilon, \bar{\sigma}=\sigma$ ). However this symmetry generally involves nontrivial phase factors, so we find the brute-force calculation a safer approach.

Let us now examine consistency between fusion and braiding. Braiding is fully characterized by the action of the counterclockwise rotation on fusion spaces: $\widehat{R}_{z}^{x y}: V_{z}^{x y} \rightarrow V_{z}^{y x}$. Since in our case the spaces $V_{z}^{x y}$ and $V_{z}^{y x}$ are one-dimensional, the linear map $\widehat{R}_{z}^{x y}$ is given by a single matrix element: ${ }^{8}$

$$
\widehat{R}_{z}^{x y}\left|\psi_{z}^{x y}\right\rangle=R_{z}^{x y}\left|\psi_{z}^{y x}\right\rangle, \quad \text { where } \quad R_{z}^{x y}=\left\langle\psi_{z}^{y x}\left|\widehat{R}_{z}^{x y}\right| \psi_{z}^{x y}\right\rangle
$$

In graphic notation, $\widehat{R}_{z}^{x y}\left|\psi_{z}^{x y}\right\rangle=\gamma_{\mathrm{z}}^{\mathrm{y} y}$, therefore Eq. (87) reads:

$$
\sum_{z}^{\mathrm{y}}=\left.R_{z}^{x y}\right|_{\mathrm{z}} ^{\mathrm{y}}
$$

Nontrivial relations arise if we consider the action of braiding operators on the fusion space of three particles. These relations can be expressed by two commutative diagrams called the hexagon equations, see Fig. 20 on page 81. The arrows in those diagrams may be understood as equalities of vectors in the space $V_{u}^{y z x}$. Let us consider the following example of the first equation (the first line below corresponds to the upper path across the hexagon, the second to the lower path):

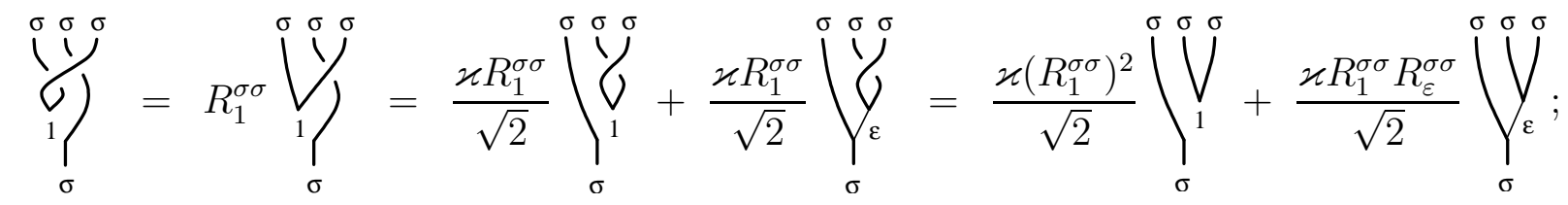

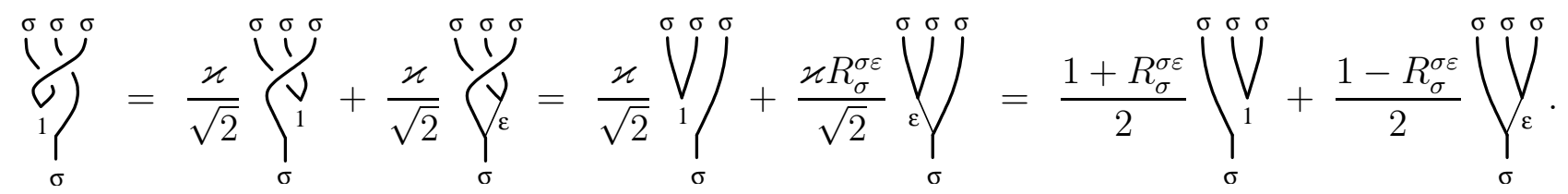

Thus we get: $1+R_{\sigma}^{\sigma \varepsilon}=\varkappa \sqrt{2}\left(R_{1}^{\sigma \sigma}\right)^{2}, 1-R_{\sigma}^{\sigma \varepsilon}=\varkappa \sqrt{2} R_{1}^{\sigma \sigma} R_{\varepsilon}^{\sigma \sigma}$.

The full set of equations arising from the first hexagon is as follows:

$$
V_{\sigma}^{\sigma \sigma \sigma}:\left\{\begin{array}{l}
1+R_{\sigma}^{\sigma \varepsilon}=\varkappa \sqrt{2}\left(R_{1}^{\sigma \sigma}\right)^{2}, \\
1-R_{\sigma}^{\sigma \varepsilon}=\varkappa \sqrt{2} R_{1}^{\sigma \sigma} R_{\varepsilon}^{\sigma \sigma}, \\
1+R_{\sigma}^{\sigma \varepsilon}=-\varkappa \sqrt{2}\left(R_{\varepsilon}^{\sigma \sigma}\right)^{2},
\end{array} \quad V_{\varepsilon}^{\varepsilon \varepsilon \varepsilon}:\left(R_{\varepsilon}^{\varepsilon \varepsilon}\right)^{2}=1 ;\right.
$$

\footnotetext{
${ }^{8}$ In Appendix 国 we take the liberty to omit the hat from the notation $\widehat{R}_{z}^{x y}$. This should not cause confusion because we do not consider matrix elements there.
} 


$$
\begin{array}{rlll}
V_{1}^{\varepsilon \sigma \sigma}, V_{1}^{\sigma \varepsilon \sigma}: & R_{\sigma}^{\sigma \varepsilon} R_{\varepsilon}^{\sigma \sigma}=R_{1}^{\sigma \sigma}, & V_{1}^{\sigma \sigma \varepsilon}: & \left(R_{\sigma}^{\varepsilon \sigma}\right)^{2}=R_{1}^{\varepsilon \varepsilon}, \\
V_{\varepsilon}^{\varepsilon \sigma \sigma}, V_{\varepsilon}^{\sigma \varepsilon \sigma}: & R_{\sigma}^{\sigma \varepsilon} R_{1}^{\sigma \sigma}=-R_{\varepsilon}^{\sigma \sigma}, & V_{\varepsilon}^{\sigma \sigma \varepsilon}: & -\left(R_{\sigma}^{\varepsilon \sigma}\right)^{2}=1, \\
V_{\sigma}^{\sigma \varepsilon \varepsilon}, V_{\sigma}^{\varepsilon \sigma \varepsilon}: & R_{1}^{\varepsilon \varepsilon}=-1, & V_{\sigma}^{\varepsilon \varepsilon \sigma}: & -\left(R_{\sigma}^{\sigma \varepsilon}\right)^{2}=1 .
\end{array}
$$

The second hexagon is obtained from the first by replacing $R_{z}^{x y}$ with $\left(R^{-1}\right)_{z}^{x y}=\left(R_{z}^{y x}\right)^{-1}$. For example, the equation $R_{\sigma}^{\sigma \varepsilon} R_{\varepsilon}^{\sigma \sigma}=R_{1}^{\sigma \sigma}$ becomes $\left(R_{\sigma}^{\varepsilon \sigma}\right)^{-1}\left(R_{\varepsilon}^{\sigma \sigma}\right)^{-1}=\left(R_{1}^{\sigma \sigma}\right)^{-1}$.

Eliminating redundancies, we get this system of equations:

$$
R_{1}^{\varepsilon \varepsilon}=-1, \quad R_{\sigma}^{\sigma \varepsilon}=R_{\sigma}^{\varepsilon \sigma}= \pm i, \quad\left(R_{1}^{\sigma \sigma}\right)^{2}=\frac{\varkappa\left(1+R_{\sigma}^{\sigma \varepsilon}\right)}{\sqrt{2}}, \quad R_{\varepsilon}^{\sigma \sigma}=-R_{\sigma}^{\sigma \varepsilon} R_{1}^{\sigma \sigma}
$$

where $\varkappa= \pm 1$. The system has eight solutions, which all fit Eq. (170). Specifically, the solutions have the form

$$
R_{1}^{\varepsilon \varepsilon}=-1, \quad R_{\sigma}^{\sigma \varepsilon}=R_{\sigma}^{\varepsilon \sigma}=i \alpha, \quad R_{1}^{\sigma \sigma}=\theta e^{i \alpha \pi / 4}, \quad R_{\varepsilon}^{\sigma \sigma}=\theta e^{-i \alpha \pi / 4}
$$

where the following combinations of $\theta, \alpha$, and $\varkappa$ are possible:

\begin{tabular}{|c||c|c|c|c|c|c|c|c|}
\hline$\theta$ & $e^{-7 i \pi / 8}$ & $e^{-5 i \pi / 8}$ & $e^{-3 i \pi / 8}$ & $e^{-i \pi / 8}$ & $e^{i \pi / 8}$ & $e^{3 i \pi / 8}$ & $e^{5 i \pi / 8}$ & $e^{7 i \pi / 8}$ \\
\hline$\alpha$ & -1 & +1 & -1 & +1 & -1 & +1 & -1 & +1 \\
\hline$\varkappa$ & +1 & -1 & -1 & +1 & +1 & -1 & -1 & +1 \\
\hline
\end{tabular}

Thus the properties of anyons are defined by the value of $\theta$, which satisfies $\theta^{8}=-1$.

\subsection{Final details}

To choose the the right solution and to complete the description of non-Abelian anyons, we use Eq. (62) in conjunction with some general results from Appendix E

The matrix element $a_{11}=\left\langle\eta_{1} \mid \xi_{1}\right\rangle$ (cf. Eqs. (80), (81) , (866)) is equal to $\varkappa / \sqrt{2}$. But according to Eq. (187) on page [74, $a_{11}=\varkappa_{\sigma} / d_{\sigma}$, where $d_{\sigma}$ is the quantum dimension of the $\sigma$-particle, and $\varkappa_{\sigma}$ is the Frobenius-Schur indicator. Therefore

$$
d_{\sigma}=\sqrt{2}, \quad \varkappa_{\sigma}=\varkappa .
$$

The topological spin of the $\sigma$-particle can be computed using Eq. (211) on page 82 ,

$$
\theta_{\sigma}=d_{\sigma}^{-1}\left(R_{1}^{\sigma \sigma}+R_{\varepsilon}^{\sigma \sigma}\right)=\theta
$$

This result also follows from Eq. (215). Matching it with the expression of $\theta_{\sigma}$ in terms of the Chern number, we represent all eight cases in (93) by these formulas:

$$
\theta=e^{i \pi \nu / 8}, \quad \alpha=(-1)^{(\nu+1) / 2}, \quad \varkappa=(-1)^{\left(\nu^{2}-1\right) / 8} .
$$

Thus we have obtained almost all properties in Table 1]. The remaining structure, namely the topological $S$-matrix, can be found using general rules (see Appendix E). 
Superselection sectors: 1 (vacuum), $\varepsilon$ (fermion), $\quad e, m$ (vortices).

Quantum dimension: $\quad d_{1}=1, \quad d_{\varepsilon}=1 \quad d_{e}=d_{m}=1$;

Topological spin: $\quad \theta_{1}=1, \quad \theta_{\varepsilon}=-1, \quad \theta_{e}=\theta_{m}=\theta=\exp \left(\frac{\pi}{8} i \nu\right)$;

Frobenius-Schur indicator: $\quad \varkappa_{1}=1, \quad \varkappa_{\varepsilon}=1, \quad \varkappa_{e}=\varkappa_{m}=\varkappa=\exp \left(\frac{\pi}{4} i \nu\right)$.

\section{Fusion rules:}

$$
\varepsilon \times \varepsilon=1, \quad \varepsilon \times e=m, \quad \varepsilon \times m=e, \quad e \times e=m \times m=1, \quad e \times m=\varepsilon .
$$

Case 1: $\quad \nu \equiv 0,8(\bmod 16) ; \quad \theta= \pm 1, \quad \varkappa=1$.

Associativity relations: all associativity relations are trivial.

\section{Braiding rules:}

$$
\begin{array}{cll}
R_{1}^{\varepsilon \varepsilon}=-1, & R_{m}^{e \varepsilon}=1, & R_{m}^{\varepsilon e}=-1, \\
R_{1}^{e e}=R_{1}^{m m}=\theta, & R_{e}^{\varepsilon m}=1, & R_{e}^{m \varepsilon}=-1, \\
& R_{\varepsilon}^{e m}=\theta, & R_{\varepsilon}^{m e}=-\theta,
\end{array}
$$

Case $2: \quad \nu \equiv \pm 4(\bmod 16) ; \quad \theta= \pm i, \varkappa=-1$.

Nontrivial associativity relations:
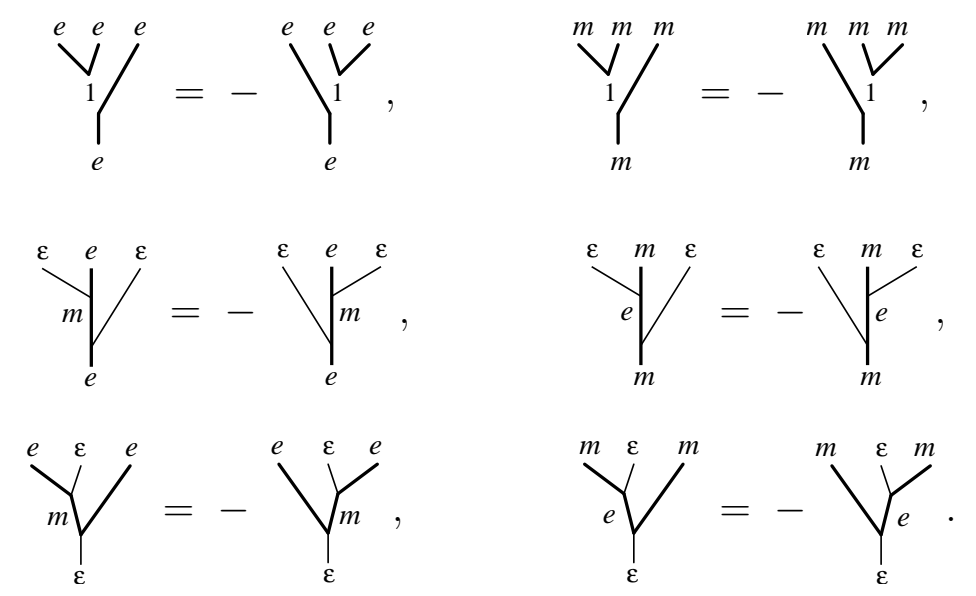

\section{Braiding rules:}

$$
\begin{array}{cc}
R_{1}^{\varepsilon \varepsilon}=-1, & R_{m}^{e \varepsilon}=R_{m}^{\varepsilon e}=R_{e}^{\varepsilon m}=R_{e}^{m \varepsilon}=\theta, \\
R_{1}^{e e}=R_{1}^{m m}=\theta, & R_{\varepsilon}^{e m}=R_{\varepsilon}^{m e}=1 .
\end{array}
$$

Table 2: Properties of anyons for $\nu \equiv 0(\bmod 4)$. 
Superselection sectors: 1 (vacuum), $\varepsilon$ (fermion), $a, \bar{a}$ (vortices).

Quantum dimension: $\quad d_{1}=1, \quad d_{\varepsilon}=1 \quad d_{a}=d_{\bar{a}}=1$;

Topological spin: $\quad \theta_{1}=1, \quad \theta_{\varepsilon}=-1, \quad \theta_{a}=\theta_{\bar{a}}=\theta=\exp \left(\frac{\pi}{8} i \nu\right)=\frac{ \pm 1 \pm i}{\sqrt{2}}$;

Frobenius-Schur indicator: $\quad \varkappa_{1}=1, \quad \varkappa_{\varepsilon}=1$.

\section{Fusion rules:}

$a \times \varepsilon=\bar{a}, \quad \bar{a} \times \varepsilon=a, \quad \varepsilon \times \varepsilon=1, \quad a \times a=\bar{a} \times \bar{a}=\varepsilon, \quad a \times \bar{a}=1$.

\section{Nontrivial associativity relations:}
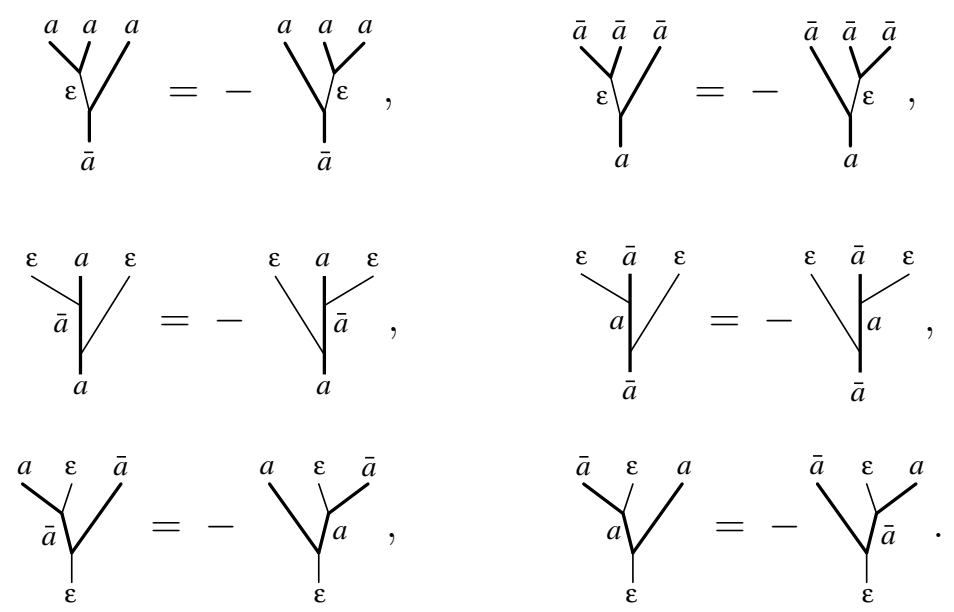

\section{Braiding rules:}

$$
\begin{array}{cc}
R_{1}^{\varepsilon \varepsilon}=-1, & R_{\bar{a}}^{a \varepsilon}=R_{\bar{a}}^{\varepsilon a}=R_{a}^{\bar{a} \varepsilon}=R_{a}^{\varepsilon \bar{a}}=\theta^{-2}, \\
R_{\varepsilon}^{a a}=R_{\varepsilon}^{\bar{a} \bar{a}}=\theta, & R_{1}^{a \bar{a}}=R_{1}^{\bar{a} a}=\theta^{-1} .
\end{array}
$$

Table 3: Properties of anyons for $\nu \equiv 2(\bmod 4)$.

\section{The sixteen-fold way}

Let us again consider the theory with $\mathbb{Z}_{2}$-vortices and free fermions whose spectrum is gapped and characterized by the Chern number $\nu$. The properties of anyons in this model depend on $\nu \bmod 16$. In the previous section we studied the case of odd $\nu$; now we assume that $\nu$ is even.

For even $\nu$, the vortices do not carry unpaired Majorana modes (see Appendix C), therefore a vortex cannot absorb a fermion while remaining in the same superselection sector. Thus, there are actually two types of vortices, which are transformed one to another by adding a fermion. Two vortices of the same type may either annihilate or fuse into a fermion. In the first case the vortices are denoted by $e$ and $m$; they obey the fusion rules (41). In the second case, we denote the vortices by $a$ and $\bar{a}$; the fusion rules are as follows:

$$
a \times \varepsilon=\bar{a}, \quad \bar{a} \times \varepsilon=a, \quad \varepsilon \times \varepsilon=1, \quad a \times a=\bar{a} \times \bar{a}=\varepsilon, \quad a \times \bar{a}=1 .
$$


Both sets of rules are Abelian, i.e., they do not contain "+" on the right-hand side.

The associativity relations can be found along the same lines as in the non-Abelian case. First, the fermions must obey the trivial relation (172). The argument used in the derivation of Eq. (71) remains valid, but we obtain two relations instead of one. Note that they are not invariant individually; for example, the sign in both relations may be changed to "+". Finally, we solve the pentagon equation. The fusion rules with $e$ and $m$ admit two nonequivalent solutions, one of which is trivial; see Table 2. Both solutions are invariant under the transformation

$$
\left|\psi_{\varepsilon}^{m e}\right\rangle \mapsto-\left|\psi_{\varepsilon}^{m e}\right\rangle, \quad\left|\psi_{m}^{e \varepsilon}\right\rangle \mapsto-\left|\psi_{m}^{e \varepsilon}\right\rangle, \quad\left|\psi_{e}^{\varepsilon m}\right\rangle \mapsto-\left|\psi_{e}^{\varepsilon m}\right\rangle
$$

(where $\mid \psi_{z}^{x y}$ is the basis vector in the corresponding fusion space), as well as trivial transformations

$$
\left|\psi_{z}^{x y}\right\rangle \mapsto \zeta_{x} \zeta_{y} \zeta_{z}^{-1}\left|\psi_{z}^{x y}\right\rangle
$$

In the case of $a$ and $\bar{a}$, there is only one solution, which doesn't admit any nontrivial symmetry; see Table 3 .

The braiding rules are found by solving the hexagon equations in conjunction with the requirement that $R_{1}^{\varepsilon \varepsilon}=-1$ and that transporting a fermion around a vortex is described by the multiplication by -1 . Then we compute the topological spin of the vortex and identify each solution with a particular value of $\nu \bmod 16$. The results are summarized in Tables 2 and 3 , (We have eliminated redundant solutions that can be obtained by the transformation (98)).

Let us mention a couple of interesting properties of these Abelian theories. For $\nu \equiv 8$ $(\bmod 16)$, all three nontrivial particles $(e, m$, and $\varepsilon)$ are fermions. For $\nu \equiv \pm 4(\bmod 16)$, the particles $e$ and $m$ are semions with trivial mutual braiding. (In comparison, the well-known Kalmeyer-Laughlin state [69] has one semionic species.)

\section{Odds and ends}

What follows are some open questions, as well as thoughts of how the present results can be extended.

1. Duan, Demler, and Lukin 42 proposed an optical lattice implementation of the Hamiltonian (4). It would be interesting to find a solid state realization as well. For example, the anisotropic exchange could be simulated by interaction of both lattice spins with a spin-1 atom coupled to a crystal field.

2. The weak translational symmetry breaking in the Abelian phase has some interesting consequences. A particularly unusual phenomenon takes place when the lattice has a dislocation. A particle winding around the dislocation changes its type: $e \leftrightarrow m$. Since $m=e \times \varepsilon$, the fermionic parity appears not to be conserved. To restore the conservation law, we must assume that the dislocation carries an unpaired Majorana mode. Therefore, Abelian phases can also be used for the implementation of quantum memory.

3. Chiral phases $(\nu \neq 0)$ require that the time-reversal symmetry be broken. In the present model, this is achieved by applying a magnetic field. However, a spontaneous breaking of time-reversal symmetry occurs in the presence of odd cycles in the lattice. For example, one can replace each vertex of the honeycomb lattice by a triangle. In this case, a gapped $\nu= \pm 1$ phase is realized without external magnetic field [70]. 
4. The representation of a spin by four Majorana fermions might be useful for other models, even though it does not lead to an exact solution. In particular, one can consider a variational mean-field state in which $c_{j}$ are decoupled from $b_{j}^{\alpha}$. It is unclear whether this type of states occur in Heisenberg antiferromagnets. On the triangular lattice, such a state has larger energy than the $120^{\circ}$ Neel-type state. It would be interesting if the former could be stabilized by additional interactions and quantum fluctuations.

5. Topological phases with free fermions coupled to an effective $\mathbb{Z}_{2}$ gauge field have been classified by $\nu$ mod 16 . However, this analysis does not include multilayer systems. One can argue that if the interaction between the layers is weak enough, topological particles cannot tunnel between the layers. Thus, each layer is described by one of the 16 theories studied above. In mathematical terms, we have the direct product of several unitary modular categories (UMCs). Strongly interacting layers are roughly described by $n$ fermionic species interacting with $\left(\mathbb{Z}_{2}\right)^{n}$-vortices, but a complete classification of such phases is yet to be found.

6. In addition to the particle classification, the chiral central charge $c_{-}=\nu / 2$ is an important robust characteristic, though its topological meaning is not so clear. It appears that in multilayer systems the total value of $\nu$ is shared between the layers, i.e., increasing $\nu$ by 16 in one layer while decreasing it in another does make a different topological phase. More generally, a topological phase is characterized by a UMC and a real number $c_{-}$ satisfying the relation (172). To prove or disprove this statement, a mathematical notion of equivalence between topological phases is necessary. It may be based on local (or quasilocal) isomorphisms between operator algebras.

7. A related question is whether the space-time boundary of an arbitrary topological phase can be described by a two-dimensional conformal field theory (CFT) and when two such theories have the same topological content. Two CFTs may be considered topologically equivalent if there is a consistent theory for a one-dimensional boundary between them. Conjecturally, this is the case if and only if both CFTs have the same chiral central charge $c_{-}=c-\bar{c}$ and correspond to the same UMC.

8. Another topic to study is Bose-condensation of vortices, which occurs when a vortex energy becomes negative due to some parameter change. The condensation of $e$-particles in the $\nu=0$ phase is equivalent to the confinement of $m$ - and $\varepsilon$-particles. Thus, the topological order is destroyed. In the $\nu=16$ system, this process produces a phase without anyons or fermions, but with the chiral central charge $c_{-}=8$. Under special circumstances, the boundary of this phase is described by the $E_{8}$ CFT at level 1, though a generic (nonconformal) perturbation drives it into a state with 8 chiral bosons propagating with different velocities.

9. The condensation of $\varepsilon \varepsilon$-pairs in two adjacent $\nu=1$ layers leads to the binding of singlelayer vortices into $\sigma \sigma$ pairs, which are equivalent to $a$ or $\bar{a}$ in the $\nu=2$ phase. Thus the direct product of two $\nu=1$ theories is related to the $\nu=2$ theory. It would be interesting to have a general mathematical characterization of such relations. The notion of lax tensor functor (see Remark E.26 on page 101) can be useful in this regard. 


\section{Appendix A: Numerical results on the stability of the vortex-free phase}

The goal of this study is to compare the energy of the vortex-free phase with that of other phases. The case $J_{x}=J_{y}=J_{z}=1$ has been investigated most carefully. The energy of the vortex-free phase equals $E_{0} \approx-1.5746$ per unit cell (i.e., per two sites, or one hexagon). The actual computation was done for tori with periodic or antiperiodic boundary conditions in each direction. The vortex-free phase and other periodic phases with small period are computationally very simple, so dealing with large tori is not a problem. However, the energy calculation for an arbitrary vortex configuration requires finding the singular values of an $N \times N$ matrix, where $N$ is the number of unit cells. With our setup (MATLAB on a PC) we could handle matrices of size $N \leqslant 2500$, which corresponds to systems of linear size $L=\sqrt{N} \leqslant 50$. Although these numbers are pretty large, finite-size effects are still appreciable due to the gapless nature of the spectrum (see Section 4 ).

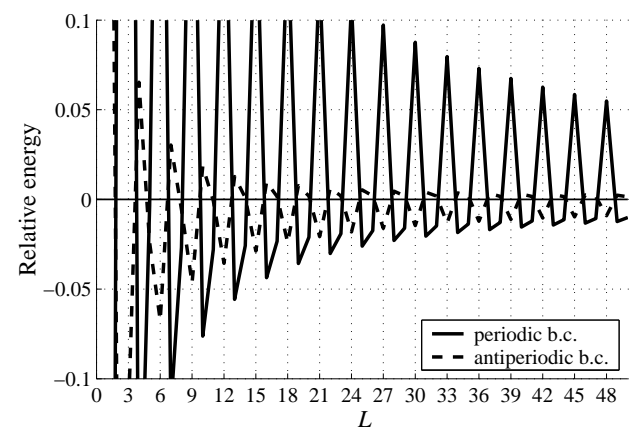

a) tori with basis $\left(L \mathbf{n}_{1}, L \mathbf{n}_{2}\right)$

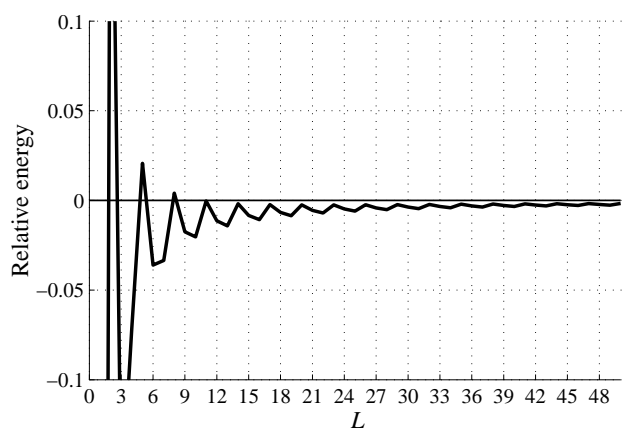

b) tori with basis $\left(L \mathbf{n}_{1}, L \mathbf{n}_{2}+\mathbf{n}_{1}\right)$

Figure 11: Finite size effects in the vortex-free phase.

Let us first discuss the finite-size effects in the vortex-free phase. From now on, we consider the relative energy, i.e., we subtract $N E_{0}$ from actual results. The plot in Fig. 11] shows the relative energy as a function of size for symmetric $L \times L$ tori. The oscillatory behavior with period 3 is due the fact that $\varepsilon(\mathbf{q})$ vanishes at $\mathbf{q}=\mathbf{q}_{*}$ (see (34)). One may argue that each period $\mathbf{r}$ of the torus contributes $\sim|\mathbf{r}|^{-1} \cos \left(2\left(\mathbf{q}_{*}, \mathbf{r}\right)\right)$ to the total energy (there are infinitely many such terms since the periods form a lattice). Interestingly enough, these contributions almost cancel each other for periodic boundary conditions on the torus with basis $\left(L \mathbf{n}_{1}, L \mathbf{n}_{2}+\mathbf{n}_{1}\right)$, where $\mathbf{n}_{1}$ and $\mathbf{n}_{2}$ are the basis vectors of the lattice (see figure in Eq. (32)). The corresponding plot is shown in Fig. 11p.

The energy of an isolated vortex is $E_{\text {vortex }} \approx 0.1536$ above the ground state. ${ }^{9}$ The calculation was done for tori with basis $\left(L \mathbf{n}_{1}, L \mathbf{n}_{2}+\mathbf{n}_{1}\right)$ for $L=9, \ldots, 32$. (We actually put 4 vortices on the torus of twice this size because the number of vortices must be even). Then the results were extrapolated to $L=\infty$ by fitting the curve $E(L)=E_{\mathrm{vortex}}+a_{1} L^{-1}+a_{2} L^{-2}$ to the data, separately for $L=3 k, L=3 k+1$, and $L=3 k+2$ (see Fig. 12).

\footnotetext{
${ }^{9}$ No rigorous precision analysis was attempted, but the error in this and the other figures is expected not to exceed 1-2 units of the least significant digit.
} 


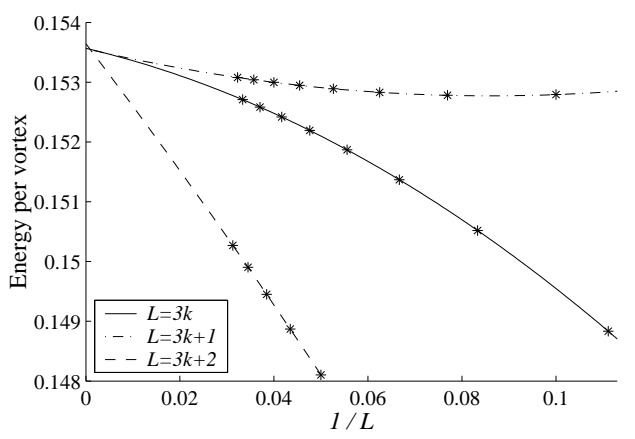

Figure 12: Extrapolation to infinite size: 4 equally spaced vortices on the torus with basis $\left(2 L \mathbf{n}_{1}, 2\left(L \mathbf{n}_{2}+\mathbf{n}_{1}\right)\right)$.

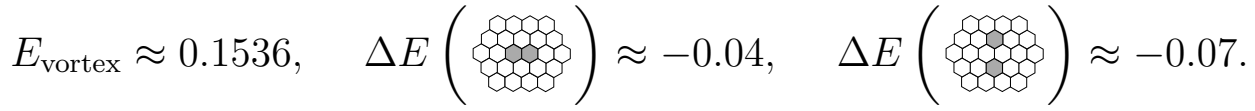

\begin{tabular}{|c|c|c|c|}
\hline & Phase & $\begin{array}{l}\text { Vortex } \\
\text { density }\end{array}$ & $\begin{array}{l}\text { Energy } \\
\text { per } \oslash \text { and } \\
\text { per vortex }\end{array}$ \\
\hline \multirow{2}{*}{1} & & 1 & 0.067 \\
\hline & & $\overline{1}$ & 0.067 \\
\hline \multirow{2}{*}{2} & & 1 & 0.052 \\
\hline & & $\overline{2}$ & 0.104 \\
\hline \multirow{2}{*}{3} & & 1 & 0.041 \\
\hline & & $\overline{3}$ & 0.124 \\
\hline \multirow{2}{*}{4} & & 2 & 0.054 \\
\hline & & $\overline{3}$ & 0.081 \\
\hline \multirow{2}{*}{5} & & 1 & 0.026 \\
\hline & & $\overline{3}$ & 0.078 \\
\hline \multirow{2}{*}{6} & & 2 & 0.060 \\
\hline & & $\overline{3}$ & 0.090 \\
\hline \multirow{2}{*}{7} & & 1 & 0.034 \\
\hline & & $\overline{4}$ & 0.136 \\
\hline
\end{tabular}

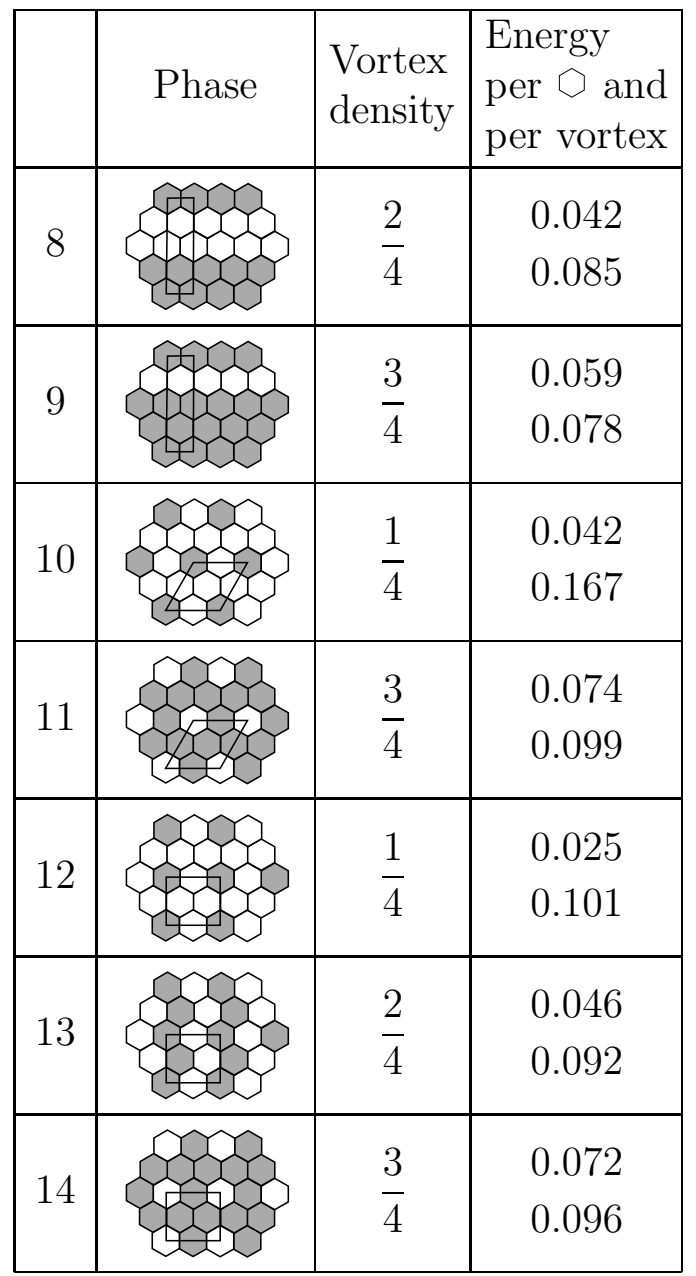

Table 4: Numerical results for $J_{x}=J_{y}=J_{z}=1$. Vortices are shown in gray; for periodic phases the unit cell is indicated by a parallelogram. 
The energy of a vortex pair is smaller than $2 E_{\text {vortex }}$ if the vortices are close to each other: by $\approx 0.04$ for nearest neighbors and by $\approx 0.07$ for next-nearest neighbors. (We didn't try to compensate the finite size effects, so the precision is poor). These numbers suggest that inter-vortex interaction is rather strong and could, in principle, result in some configurations having negative energy. However, our further calculations give evidence for the contrary.

We have checked all periodic phases with unit cell containing 1,2, 3 or 4 hexagons, see Table 4. (As mentioned above, this computation requires much less computer resources than the study of separate vortices or vortex pairs). In all these cases the energy is positive and increases as more vortices are added. The smallest energy per vortex is achieved by phases 1 and $5-0.067$ and 0.078 , respectively.

All 14 phases have positive energies (relative to the vortex-free phase) for all nonzero values of $J_{x}, J_{y}, J_{z}$.

\section{Appendix B: Edge modes in phases $B_{\nu}$}

It is understood that the edge spectrum depends strongly on particular conditions at the edge. The calculations below are only meant to illustrate the universal feature of the spectrum the existence of a chiral gapless mode.

Let us suppose that the honeycomb lattice fills the lower half-plane and is cut as follows:

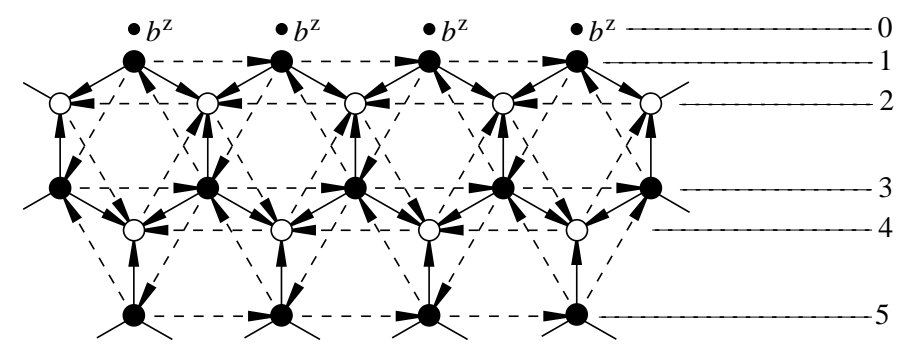

Row 0 consists of the Majorana operators $b_{j}^{z}$, which would be decoupled from the rest of the system if not for the magnetic field. Performing the Fourier transform in the horizontal direction, we compute effective couplings between the rows as functions of $q_{x}$ :

$$
i A\left(q_{x}\right)=\left(\begin{array}{ccccccc}
0 & i \gamma & 0 & & & & \\
-i \gamma & \alpha & i s & -\beta & & & \\
0 & -i s & -\alpha & i r & \beta & & \\
& -\beta & -i r & \alpha & i s & -\beta & \\
& & \beta & -i s & -\alpha & i r & \ddots \\
& & & -\beta & -i r & \alpha & \ddots \\
& & & & \ddots & \ddots & \ddots
\end{array}\right), \quad \text { where } \quad \begin{aligned}
& \\
& \\
&
\end{aligned}
$$

Let us first find edge modes ignoring the operators $b_{j}^{z}$, i.e., the first row and column in the above matrix. If $\kappa=0$, then for $2 \pi / 3<q_{x}<4 \pi / 3$ the matrix has a null vector with elements $\psi(2 j)=0$ and $\psi(2 j+1)=\left(-2 \cos \frac{q_{x}}{2}\right)^{j}$, which corresponds to a zero-energy state localized near the edge. If $\kappa$ is not zero but still small, we get this spectrum:

$$
\varepsilon\left(q_{x}\right) \approx 12 \kappa \sin q_{x}, \quad q_{x} \in[2 \pi / 3,4 \pi / 3] .
$$




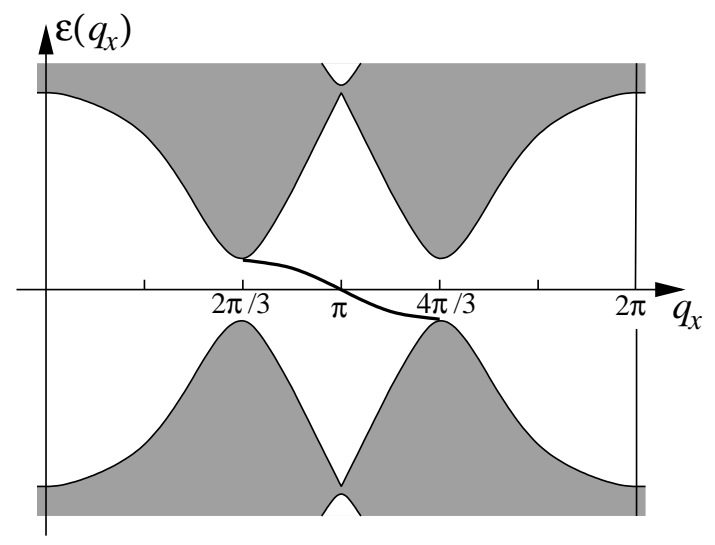

a)

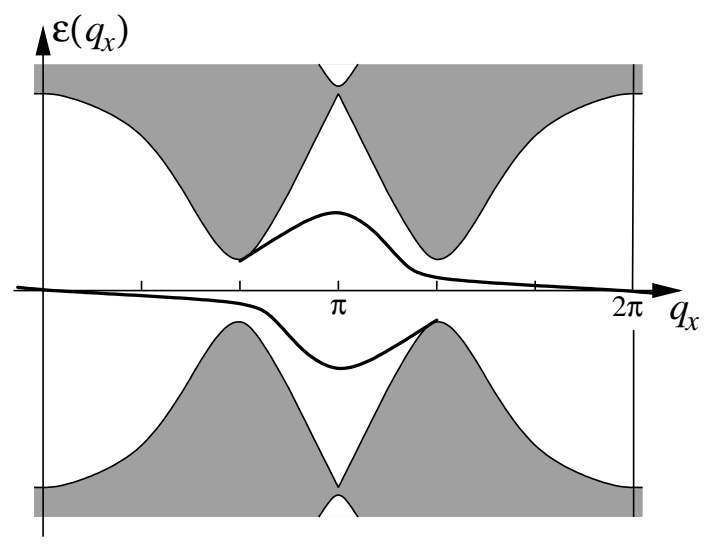

b)

Figure 13: Schematic form of the edge spectrum in the simplest case (a) and with the operators $b_{j}^{z}$ taken into account (b). The shaded area represents the bulk spectrum.

It is shown in Fig. 13a, assuming that $\kappa>0$, i.e., $\nu=+1$. The point where the energy curve crosses zero, $q_{x}=\pi$, corresponds to a left-moving gapless mode.

Now we take the operators $b_{j}^{z}$ into account. If $\kappa=0$, then a zero mode exists for $q_{x} \in$ $[-2 \pi / 3,2 \pi / 3]$, whereas for $q_{x} \in[2 \pi / 3,4 \pi / 3]$ there are two modes with energies

$$
\varepsilon\left(q_{x}\right) \approx \pm \sqrt{1-4 \cos ^{2} \frac{q_{x}}{2}}
$$

The spectrum for $\kappa>0$ is shown schematically in Fig. 13b. In this case a left-moving gapless mode occurs at $q_{x}=0$.

In conclusion, let us give a simple (but not completely rigorous) proof of Eq. (56). The idea is due to Laughlin [71]: we put the system on a cylinder and run magnetic flux through it. For simplicity, we consider a cylinder of the smallest radius, which is equal to the lattice period divided by $2 \pi$. Thus the Hamiltonian is given by the matrix $i A\left(q_{x}\right)$; the variable $q_{x}$ plays the role of the magnetic flux.

Let $q_{x}$ vary from 0 to $2 \pi$. When the energy of an edge state ${ }^{10}|\psi\rangle$ crosses zero, the spectral projector $P\left(q_{x}\right)$ changes by $|\psi\rangle\langle\psi|$. For an arbitrary operator $Q$ the quantity $\operatorname{Tr}\left(Q P\left(q_{x}\right)\right)$ changes by $\langle\psi|Q| \psi\rangle$. Let

$$
Q_{j k}=g(j) \delta_{j k}, \quad g(j)= \begin{cases}1 & \text { near the edge } \\ 0 & \text { far away from the edge }\end{cases}
$$

(the exact form of the function $g$ is not important). Then $\langle\psi|Q| \psi\rangle \approx 1$. Since $\operatorname{Tr}\left(Q P\left(q_{x}\right)\right)$ is a periodic function of $q_{x}$, the abrupt changes must be compensated by a continuous variation, which we call "adiabatic". Thus

$$
\nu_{\text {edge }}=\int I\left(q_{x}\right) d q_{x}, \quad \text { where } \quad I\left(q_{x}\right)=-\operatorname{Tr}(Q \dot{P}), \quad \dot{P}=\left(\frac{d P}{d q_{x}}\right)_{\text {adiabatic }} .
$$

\footnotetext{
${ }^{10}$ We are using the first quantization formalism, therefore "states" are just superpositions of lattice points.
} 
The adiabatic evolution of the spectral projector may be represented by the Heisenberg equation with a suitable Hamiltonian:

$$
\dot{P}=i[H, P], \quad \text { where } H=i[P, \dot{P}] \text {. }
$$

Indeed, $P^{2}=P$, hence $P \dot{P}+\dot{P} P=\dot{P}$ and $P \dot{P} P=0$, implying that $-[[P, \dot{P}], P]=\dot{P}$. Therefore

$$
I\left(q_{x}\right)=-i \operatorname{Tr}(Q H P-Q P H)=-i \operatorname{Tr}(H P Q-H Q P)=-i \operatorname{Tr}(H[P, Q]) .
$$

(These transformations are valid because each trace is represented by a finite sum.) The last expression in Eq. (106) may be calculated using the spectral projector for the bulk. Indeed, if the function $g$ were constant, the commutator $[P, Q]$ would vanish; thus the main contribution comes from the region where $g$ changes from 1 to 0 .

In the final part of the proof, we take into account the periodicity of the bulk spectral projector, representing the site index $j$ as $(s, \lambda)$. As is usual, lattice cells are indexed by $s$; it is an integer that increases in the positive $y$-direction. We may assume that the function $g$ depends only on $s$ and satisfies $\lim _{s \rightarrow \infty} g(s)=1, \lim _{s \rightarrow-\infty} g(s)=0$. Then

$$
([P, Q])_{t \mu, s \lambda}=(g(s)-g(t)) P_{t \mu, s \lambda}, \quad \sum_{t}(g(t+r)-g(t))=r .
$$

Using these auxiliary identities, we pass to the momentum representation:

$$
\begin{aligned}
\operatorname{Tr}(H[P, Q]) & =\sum_{s, \lambda, t, \mu} H_{s \lambda, t \mu} P_{t \mu, s \lambda}(g(s)-g(t))=\sum_{r, \lambda, \mu} H_{0 \lambda,-r \mu} P_{0 \mu, r \lambda} \sum_{t}(g(t+r)-g(t)) \\
& =\sum_{r, \lambda, \mu} H_{0 \lambda,-r \mu} P_{0 \mu, r \lambda} r=-i \int \operatorname{Tr}\left(\widetilde{H} \frac{\partial \widetilde{P}}{\partial q_{y}}\right) \frac{d q_{y}}{2 \pi}=\int \operatorname{Tr}\left(\left[\widetilde{P}, \frac{\partial \widetilde{P}}{\partial q_{x}}\right] \frac{\partial \widetilde{P}}{\partial q_{y}}\right) \frac{d q_{y}}{2 \pi}
\end{aligned}
$$

Substituting the result into (106) and (104), we get

$$
\nu_{\text {edge }}=\int I\left(q_{x}\right) d q_{x}=\frac{-i}{2 \pi} \int \operatorname{Tr}\left(\left[\widetilde{P}, \frac{\partial \widetilde{P}}{\partial q_{x}}\right] \frac{\partial \widetilde{P}}{\partial q_{y}}\right) d q_{x} d q_{y}=\nu .
$$

\section{Appendix C: Quasidiagonal matrices}

The goal of this appendix is to provide a formal argument for the existence of unpaired Majorana modes on vortices for odd values of the Chern number $\nu$. However, the developed formalism may be interesting on its own right. It suggests a rather efficient approach to problems like quantization of Hall conductivity in disordered systems without the use of excessively heavy tools. Some of results (in particular, the ones concerning the flow of a matrix and the Chern number) are actually poor man's variants of known mathematical theorems. ${ }^{11}$ A powerful theory, called noncommutative geometry [45] was used by Bellissard at al [1] to prove rigorously that the Hall conductivity is quantized provided the electron are localized (see [72, 73] for

\footnotetext{
${ }^{11}$ Note for experts: we are effectively trying to build a K-theory on a manifold by considering functions that are not continuous, but rather constant on cells that are dual to simplices. This seems to be an awkward approach, but its possible advantage is the relation to a second-quantized case, see Appendix D.
} 


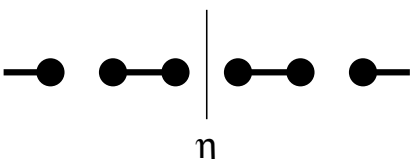

a)

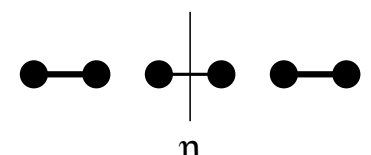

b)

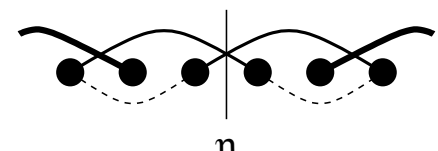

$\mathrm{n}$

c)

Figure 14: Cutting a Majorana chain: $\mathcal{M}_{\eta}(B)=1$ in cases (a) and (c) whereas $\mathcal{M}_{\eta}(B)=-1$ in case (b). The dashed lines in (c) show a possible way to reconnect the broken pairs.

another proof). We will use similar ideas, but focus mainly on providing the intuition rather than mathematical rigor.

A physical example of a quasidiagonal matrix is an electron hopping matrix $T=\left(t_{j k}\right)$ on a $d$-dimensional lattice. It is a Hermitian matrix with the property that $t_{j k}$ is bounded in magnitude and vanishes if the distance $|j-k|$ between the sites $j$ and $k$ is greater that some constant $L$. Furthermore, if $T$ has a spectral gap, i.e., if the eigenvalues are bounded away from zero, then the corresponding spectral projector $P=\frac{1}{2}(1-\operatorname{sgn} T)$ is also quasidiagonal. More specifically, the matrix elements $P_{j k}$ decay exponentially with distance. ${ }^{12}$

In general, a quasidiagonal matrix is a lattice-indexed matrix $A=\left(A_{j k}\right)$ with sufficiently rapidly decaying off-diagonal elements. Technically, one requires that

$$
\left|A_{j k}\right| \leqslant c|j-k|^{-\alpha}, \quad \alpha>d,
$$

where $c$ and $\alpha$ are some constants, and $d$ is the dimension of the space. Note that "lattice" is simply a way to impose coarse $\mathbb{R}^{d}$ geometry at large distances. We may think about the problem in these terms: matrices are operators acting in some Hilbert space, and lattice points are basis vectors. But the choice of the basis need not be fixed. One may safely replace the basis vector corresponding to a given lattice point by a linear combination of nearby points. One may also use some kind of coarse-graining, replacing the basis by a decomposition into orthogonal subspaces corresponding to groups of points, or regions in $\mathbb{R}^{d}$.

Let us outline the main results. We first consider quasidiagonal unitary matrices in one dimension and define an integral topological characteristic called flow. Then we study projection matrices in two dimensions. The Chern number $\nu(P)$ of a quasidiagonal projection matrix $P$ is expressed directly in terms of the matrix elements $P_{j k}$, see Eqs. (124) and (122). This definition does not rely on translational invariance, but in the translationally invariant case we reproduce Eq. (154).

After those preliminaries, we switch to questions related to Majorana fermions (all necessary background is given in Section 31). From the mathematical point of view, we study quasidiagonal real skew-symmetric matrices $B$ satisfying the condition $B^{2}=-1$. In one dimension, such a matrix is assigned a cutting obstruction $\mathcal{M}_{\eta}(B)= \pm 1$ with respect to an arbitrary cut $\eta$ dividing the chain into two parts. Let us explain the physical meaning of this number. The matrix $B$ defines a pairing of Majorana modes (in the exact sense stated right after Eq. (27), but we will use a cartoon description for illustration). If we cut the chain, the resulting pieces may carry unpaired boundary modes 64. This happens when an odd number of pairs is broken, as

\footnotetext{
${ }^{12}$ Indeed, the function $f(x)=\operatorname{sgn} x$ can be approximated by a sequence of polynomials $p_{n}(x)$ of degree $n \rightarrow \infty$ which converges exponentially on the spectrum of $T$. Therefore $\left\|P-p_{n}(T)\right\|<a b^{n}$ for some $b<1$. On the other hand, the matrix elements $\left(p_{n}(T)\right)_{j k}$ vanish if $|j-k|>n L$.
} 


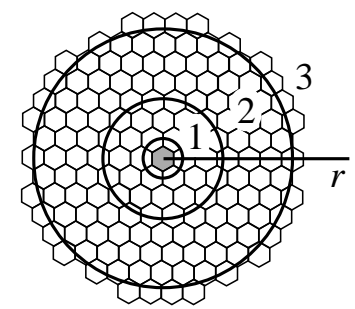

a)

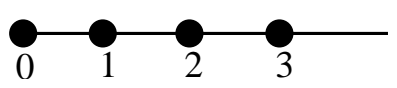

b)

Figure 15: A map from two to one dimension: annular regions around the vortex (a) are collapsed into points on a ray (b).

in Fig. 14b; otherwise one can modify the matrix near the cut so that to avoid broken pairs, see Fig. 14k. More formally, let us consider quasidiagonal real skew-symmetric matrices $B^{\prime}$ that agree with $B$ at infinity but have no nonzero elements across the cut. We prove that if $\mathcal{M}_{\eta}(B)=-1$, then no such $B^{\prime}$ exists that satisfies the equation $\left(B^{\prime}\right)^{2}=-1$. Note that while $\mathcal{M}_{\eta}(B)$ depends on the cut, the relative Majorana number of two matrices on the same chain, $\mathcal{M}(A, B)=\mathcal{M}_{\eta}(A) \mathcal{M}_{\eta}(B)$ is invariant.

The concept of Majorana number is also applicable in two dimensions. Let $B^{2}=-1$, and let us construct another matrix $B^{\prime}$ by inserting a $\mathbb{Z}_{2}$-vortex at the origin. More exactly, $B_{j k}^{\prime}= \pm B_{j k}$, where the minus sign occurs when the link $(j, k)$ crosses some fixed ray $r$ (an analogue of the Dirac string). Note that the condition $\left(B^{\prime}\right)^{2}=-1$ is only true asymptotically (i.e., far away from the origin); it may or may not be possible to satisfy this equation by altering the matrix elements near the vortex. In fact, the presence of an unpaired Majorana mode at the vortex is defined as locally unrepairable failure of the equation $\left(B^{\prime}\right)^{2}=-1$. Such a mode is detected by an absolute Majorana number $\mathcal{M}\left(B^{\prime}\right)$. For its construction, let us regard $B$ and $B^{\prime}$ as one-dimensional by keeping track of the radial direction only: we divide the plane into concentric rings and map all sites in each ring to a single location on a ray (see Fig. 15). To simplify the calculation, we cut out the interior of a sufficiently large circle $\eta$; let $|\eta|$ be the number of sites removed. The presence or absence of an unpaired mode in this case is given by $\mathcal{M}_{\eta}\left(B^{\prime}\right)$, which is defined using some annular neighborhood of $\eta$. Extrapolating to $|\eta|=0$, we get $\mathcal{M}\left(B^{\prime}\right)=(-1)^{|\eta|} \mathcal{M}_{\eta}\left(B^{\prime}\right)=\mathcal{M}\left(B, B^{\prime}\right)$. The last number only depends on the matrix elements of $B$ in some neighborhood of the intersection point between $\eta$ and $r$. Finally, we show that $\mathcal{M}\left(B, B^{\prime}\right)=(-1)^{\nu(P)}$, where $\nu(P)$ is the Chern number associated with the projector $P=\frac{1}{2}(1-i B)$. Thus, vortices carry unpaired Majorana modes if and only if $\nu(P)$ is odd.

\section{C.1 The flow of a unitary matrix}

C.1.1 Definition. Let us consider an arbitrary (possibly infinite) unitary matrix $U=\left(U_{j k}\right)$. We refer to the values of $j$ and $k$ as "sites" and define a "current" flowing from $k$ to $j$ :

$$
f_{j k}=\left|U_{j k}\right|^{2}-\left|U_{k j}\right|^{2}
$$

Since $U$ is unitary, $\sum_{j} U_{j l}^{*} U_{j k}=\sum_{j} U_{l j} U_{k j}^{*}=\delta_{l k}$, therefore the current is conserved at each site:

$$
\sum_{j} f_{j k}=0
$$


Suppose that the sites are positioned on a line (more specifically, enumerated by integers), and that the off-diagonal matrix elements of $U$ decay fast enough. We will see later that the condition

$$
\left|U_{j k}\right| \leqslant c|j-k|^{-\alpha}, \quad \text { where } \alpha>1,
$$

is sufficient for our purposes. For now, let us simply assume that $U_{j k}$ vanishes if $|j-k|$ is greater than some constant $L$. Then it is obvious that the total current through a "cross section" $\eta$,

$$
\mathcal{F}(U)=\sum_{j \geqslant \eta} \sum_{k<\eta} f_{j k}
$$

does not depend on the choice of $\eta$. The number $\mathcal{F}(U)$ is called the flow of $U$. For example, the matrix with entries $U_{j k}=\delta_{j, k+s}$ has flow $s$.

C.1.2 Integrality of the flow. A nontrivial property is that the flow is quantized, i.e., has an integer value. To prove this statement, let us introduce the projector onto the sites $M$ through $N$ :

$$
\Pi_{j k}^{[M, N]}= \begin{cases}1 & \text { if } M \leqslant j=k \leqslant N \\ 0 & \text { otherwise. }\end{cases}
$$

The projector onto an infinite interval is defined similarly. Let $\Pi=\Pi^{[0, \infty)}$; then

$$
\mathcal{F}(U)=\operatorname{Tr}\left(U^{\dagger} \Pi U(1-\Pi)\right)-\operatorname{Tr}\left(U^{\dagger}(1-\Pi) U \Pi\right)=\operatorname{Tr}\left(U^{\dagger} \Pi U-\Pi\right) .
$$

(Caution: In the last expression, the order of operations is important: we first compute the matrix $\Lambda=U^{\dagger} \Pi U-\Pi$, and then its trace, $\sum_{j} \Lambda_{j j}$. It is not possible to use the cyclic property of the trace, $\operatorname{Tr} A B=\operatorname{Tr} B A$, since it is only valid if one of the matrices has a finite number of nonzero elements or, more generally, if the sum involved in the calculation of the trace converges absolutely.)

Note that the matrices $\Pi$ and $U^{\dagger} \Pi U$ are orthogonal projectors. If they were finite, their traces would be integers, and the difference would also be an integer. In the infinite case, one may refer to the general notion of the relative index of two projectors [73, 74]. However, we will proceed in a more pedestrian fashion and simply truncate the matrices.

Let $\Lambda=U^{\dagger} \Pi U-\Pi$. It is clear that the matrix element $\Lambda_{j k}$ vanishes if $|j|$ or $|k|$ is greater than $L$, so $\Lambda$ is not changed by the truncation to the interval $[-L, L]$. Hence

$$
\mathcal{F}(U)=\operatorname{Tr}\left(U^{\dagger} \Pi U\right)_{\text {trunc }}-\operatorname{Tr} \Pi_{\text {trunc }}, \quad \text { where } A_{\text {trunc }} \stackrel{\text { def }}{=} \Pi^{[-L, L]} A \Pi^{[-L, L]} .
$$

If $A$ is an orthogonal projector and commutes with $\Pi^{[-L, L]}$, then $A_{\text {trunc }}$ is also an orthogonal projector. Obviously, $\Pi$ and $\Lambda$ commute with $\Pi^{[-L, L]}$, and so does the matrix $U^{\dagger} \Pi U=\Pi+\Lambda$. Thus both $\Pi_{\text {trunc }}$ and $\left(U^{\dagger} \Pi U\right)_{\text {trunc }}$ are orthogonal projectors. It follows that $\mathcal{F}(U)$ is an integer.

In equation (112), the projector $\Pi$ may be replaced by another operator with the same asymptotics at infinity. (In other words, we can "blur" the boundary between the left and the right half-line.) Indeed, the expression $\operatorname{Tr}\left(U^{\dagger} A U-A\right)$ vanishes if the cyclic property of the trace is true, i.e., if the elements of $A$ decay at infinity fast enough. Adding such a matrix $A$ to $\Pi$ will not change the result. Thus

$$
\mathcal{F}(U)=\operatorname{Tr}\left(U^{\dagger} Q U-Q\right)=\operatorname{Tr}\left(U^{\dagger}[Q, U]\right), \quad \text { where } \quad Q_{j k}= \begin{cases}\delta_{j k} & \text { for } j, k \rightarrow+\infty \\ 0 & \text { for } j, k \rightarrow-\infty\end{cases}
$$


(Here we also assume that the matrix $Q$ is quasidiagonal, i.e, $\left|Q_{j k}\right|$ is bounded by a rapidly decaying function of $|j-k|$, cf. (109) $)$ )

C.1.3 Translationally-invariant case. The flow can be easily calculated if $U$ possesses a translational symmetry. Let us group the sites by unit cells and index them as $(s, \lambda)$, where $s$ is the number of the cell, and $\lambda$ refers to a position type inside the cell. We assume that $U_{s \lambda, t \mu}$ depends only on $t-s, \lambda$ and $\mu$. In this case, we can express $\mathcal{F}(U)$ in terms of the trace per unit cell (denoted by tr) and a position operator $X$ :

$$
\mathcal{F}(U)=\operatorname{tr}\left(U^{\dagger}[X, U]\right), \quad \text { where } X_{s \lambda, t \mu}=s \delta_{s t} \delta_{\lambda \mu} .
$$

(For a proof, replace the factor $s$ in the definition of $X$ by the function $g(s)$ such that $g(s)=s$ for $|s| \leqslant N$ and $g(s)=N \operatorname{sgn} s$ for $|s|>N$, where $N$ is large; then use Eq. (114)).

In the momentum representation, the operator $U$ becomes $\widetilde{U}_{\mu \lambda}(q)=\sum_{t} e^{i(\mathbf{q}, t)} U_{0 \lambda, t \mu}$. We may also use these simple rules:

$$
[X, A] \rightarrow i \frac{d \tilde{A}}{d q}, \quad \operatorname{tr} A \rightarrow \int_{-\pi}^{\pi} \frac{d q}{2 \pi} \operatorname{Tr} \widetilde{A}
$$

Thus

$$
\mathcal{F}(U)=\frac{i}{2 \pi} \int_{-\pi}^{\pi} \operatorname{Tr}\left(\widetilde{U^{\dagger}} \frac{d \widetilde{U}}{d q}\right) d q=-\frac{1}{2 \pi i} \int_{q=-\pi}^{q=\pi} d(\ln \operatorname{det} \widetilde{U}(q))
$$

\section{C.2 General setting}

The result about the flow quantization can be extended to matrices satisfying condition (109), which guarantees that the sum in Eq. (110) converges absolutely.

Another generalization is coarse-graining, which means to allow multiple states per site. The sites are now described by orthogonal subspaces of the total Hilbert space. This generalization is useful if want to treat a two-dimensional system as one-dimensional.

Finally, we may try to apply the notion of flow to finite systems. Let us assume that the unitarity condition, $\sum_{j} U_{j l}^{*} U_{j k}=\sum_{j} U_{l j} U_{k j}^{*}=\delta_{l k}$, is approximate and holds only if $j$ and $k$ belong to some interval. In this case, one needs to restrict the sum (110) to the same interval, and the result will not be an exact integer. But one can actually give an upper bound for the deviation from the closest integer.

Setting the last generalization aside, let us put the integrality theorem into a rigorous form. (The reader may safely skip this formalism and proceed to the next subsection.)

Theorem C.1. Let $U$ be a unitary operator acting in the Hilbert space $\mathcal{H}=\bigoplus_{j=-\infty}^{\infty} \mathcal{H}_{j}$ and represented by a matrix whose entries $U_{j k}$ are linear maps from $\mathcal{H}_{k}$ to $\mathcal{H}_{j}$. Suppose that the off-diagonal entries are Hilbert-Schmidt operators satisfying the condition

$$
\left\|U_{j k}\right\|_{\mathrm{HS}} \leqslant c|j-k|^{-\alpha}
$$

where $c$ and $\alpha>1$ are some constants, and $\|\cdot\|_{\mathrm{HS}}$ is the Hilbert-Schmidt norm. Then the sum in the expression for the flow,

$$
\mathcal{F}(U)=\sum_{j \geqslant 0} \sum_{k<0}\left(\operatorname{Tr}\left(U_{j k}^{\dagger} U_{j k}\right)-\operatorname{Tr}\left(U_{k j}^{\dagger} U_{k j}\right)\right)
$$

converges absolutely and has an integer value. 
Proof sketch. Let $\mathcal{H}_{-}=\bigoplus_{j<0} \mathcal{H}_{j}$ and $\mathcal{H}_{+}=\bigoplus_{j \geqslant 0} \mathcal{H}_{j}$. An operator $A$ is said to be quasitrace-class relative to the decomposition $\mathcal{H}=\mathcal{H}_{-} \oplus \mathcal{H}_{+}$if the matrix elements $A_{++}$and $A_{--}$are trace class, whereas $A_{+-}$and $A_{-+}$are Hilbert-Schmidt. By definition, $\operatorname{Tr} A=\operatorname{Tr} A_{++}+\operatorname{Tr} A_{--}$. An operator $B$ is said to be quasi-bounded if $B_{++}, B_{--}$are bounded and $B_{+-}, B_{-+}$are Hilbert-Schmidt. Both types of operators form Banach spaces with respect to suitable norms; quasi-trace-class operators form an ideal in the algebra of quasi-bounded operators.

One can show that the operator $\Lambda=U^{\dagger} \Pi U-\Pi$ is quasi-trace-class. Also, the operators $P(L)=\Pi^{[-L, L]} U^{\dagger} \Pi U \Pi^{[-L, L]}$ are almost projectors, in the sense that $\lim _{L \rightarrow \infty}\left(P(L)^{2}-P(L)\right)=0$ with respect to the quasi-trace norm. Hence $P(L)$ can be approximated by a projector, which has an integer trace.

We omit the details and proceed to higher dimensions. Generally, our style will not be very rigorous, but one can hopefully elaborate the results using the following definition.

Definition C.2. Let a positive integer $d$ (the dimension) and a real number $\alpha>d$ be fixed. A matrix $\left(A_{j k}: j, k \in \mathbb{Z}^{d}\right)$ with operator entries is called quasidiagonal if all its off-diagonal entries are Hilbert-Schmidt, and there are some constants $c$ and $c^{\prime}$ such that

$$
\left\|A_{j j}\right\| \leqslant c, \quad\left\|A_{j k}\right\|_{\mathrm{HS}} \leqslant c^{\prime}|j-k|^{-\alpha} \quad \text { for } j \neq k .
$$

Note that if $A$ is unitary, then $\left\|A_{j j}\right\| \leqslant 1$ automatically. Quasidiagonal matrices form a Banach algebra with the norm

$$
\|A\|_{\mathrm{qd}}=\sup _{j}\left\|A_{j j}\right\|+\gamma \sup _{j \neq k}|j-k|^{\alpha}\left\|A_{j k}\right\|_{\mathrm{HS}},
$$

where $\gamma$ is a sufficiently large constant.

\section{C.3 Chern number}

C.3.1 General definition of $\nu(P)$. Let $P$ be an orthogonal projector represented by a quasidiagonal matrix in two dimensions. (For simplicity, we assume that the matrix elements $P_{j k}$ are scalars, though they may be operators as well.) For each triple $(j, k, l)$ we define a "2-current": 13

$$
h_{j k l}=12 \pi i\left(P_{j k} P_{k l} P_{l j}-P_{j l} P_{l k} P_{k j}\right) .
$$

It is clear that $h_{j k l}$ is antisymmetric in all three indices. Since $P$ is Hermitian, $h_{j k l}$ is a real number. Moreover, since $P^{2}=P$,

$$
(\partial h)_{k l} \stackrel{\text { def }}{=} \sum_{j} h_{j k l}=0 .
$$

Let us partition the plane into three sectors and define a quantity $\nu(P)$, which will be shown to generalize the notion of the Chern number:

$$
\text { :A: } \quad \nu(P)=h(A, B, C) \stackrel{\text { def }}{=} \sum_{j \in A} \sum_{k \in B} \sum_{l \in C} h_{j k l}
$$

\footnotetext{
${ }^{13}$ Another name of this object is "simplicial 2-chain". More exactly, the 2-chain is the formal sum $\sum_{j<k<l} h_{j k l} \Delta_{j k l}$, where $\Delta_{j k l}$ is a combinatorial simplex.
} 
This sum converges absolutely. On the other hand, its value does not change if one reassigns any site from one sector to another (due to Eq. (123) $)$. Therefore $\nu(P)$ is a topological invariant: it is constant, provided that $A, B, C$ are arranged in the counterclockwise order. We will see that $\nu(P)$ is actually an integer, and its value agrees with Eq. (54) in the translationally invariant case.

C.3.2 Some properties and the translationally invariant case. Let us rewrite Eq. (124) in an operator form using the relation $A+B+C=1$, where the symbols $A, B, C$ designate the projectors on the corresponding sectors:

$$
\begin{aligned}
\nu(P) & =12 \pi i(\operatorname{Tr}(A P B P C P)-\operatorname{Tr}(A P C P B P)) \\
= & 4 \pi i \operatorname{Tr}(A P B P C P+B P C P A P+C P A P B P-A P C P B P-C P B P A P-B P A P C P) \\
& =4 \pi i \operatorname{Tr}(P A P B P-P B P A P)=4 \pi i \operatorname{Tr}[P A P, P B P] \stackrel{\text { def }}{=} \nu(P, A, B) .
\end{aligned}
$$

Note that the value of $\nu(P, A, B)$ will not change if we add arbitrary finite matrices to $A$ and $B$. Moreover, the commutator $[P A P, P B P]$ is nonzero only in the region where the supports of $A, B$, and $C=1-A-B$ touch each other, hence we can make arbitrary changes away from the triple contact point. Thus the operators $A, B, C$ do not have to be projectors or even commute with each other; it is only important that they are supported by regions with the particular topological configuration (possibly with some overlap along the boundaries and triple overlap at the center).

This freedom in the choice of $A$ and $B$ is very useful. As one application, we show that $\nu(P)$ is additive. Let $P_{1}$ and $P_{2}$ be projectors onto orthogonal subspaces (i.e., $P_{1} P_{2}=P_{2} P_{1}=0$ ) and let $P_{3}=1-P_{1}-P_{2}$. We may replace $A, B$, and $C=1-A-B$ with

$$
A^{\prime}=\sum_{k=1}^{3} P_{k} A P_{k}, \quad B^{\prime}=\sum_{k=1}^{3} P_{k} B P_{k}, \quad C^{\prime}=\sum_{k=1}^{3} P_{k} C P_{k},
$$

which differ from $A, B$, and $C$ only at the boundaries of the corresponding regions. Then

$$
\begin{gathered}
\left(P_{1}+P_{2}\right) A^{\prime}\left(P_{1}+P_{2}\right) B^{\prime}\left(P_{1}+P_{2}\right)=P_{1} A P_{1} B P_{1}+P_{2} A P_{2} B P_{2}, \\
\nu\left(P_{1}+P_{2}, A, B\right)=\nu\left(P_{1}+P_{2}, A^{\prime}, B^{\prime}\right)=\nu\left(P_{1}, A, B\right)+\nu\left(P_{2}, A, B\right) .
\end{gathered}
$$

Thus

$$
\nu\left(P_{1}+P_{2}\right)=\nu\left(P_{1}\right)+\nu\left(P_{2}\right), \quad \nu(1-P)=-\nu(P) .
$$

Now, we calculate $\nu(P, A, B)$ for a different topological topological configuration. Let $\Pi^{(x)}=$ $\Pi^{(1)}+\Pi^{(4)}$ and $\Pi^{(y)}=\Pi^{(2)}+\Pi^{(1)}$ be the projectors onto the right and the upper half-plane, respectively (see the picture below). Then

$$
\begin{aligned}
& :::: A^{y}::: \quad \nu\left(P, \Pi^{(x)}, \Pi^{(y)}\right) \\
& \because: \because::_{0}=\nu\left(P, \Pi^{(1)}, \Pi^{(2)}\right)+\nu\left(P, \Pi^{(1)}, \Pi^{(1)}\right)+\nu\left(P, \Pi^{(4)}, \Pi^{(2)}\right)+\nu\left(P, \Pi^{(4)}, \Pi^{(1)}\right)
\end{aligned}
$$

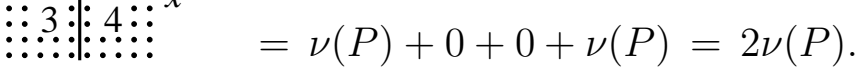

It follows that

$$
\nu(P)=2 \pi i \operatorname{Tr}\left[P \Pi^{(x)} P, P \Pi^{(y)} P\right]=2 \pi i \operatorname{Tr}\left(P\left[\left[\Pi^{(x)}, P\right],\left[\Pi^{(y)}, P\right]\right]+P\left[\Pi^{(x)}, \Pi^{(y)}\right] P\right),
$$


where the last term vanishes because $\Pi^{(x)}$ and $\Pi^{(y)}$ commute. Of course, $\Pi^{(x)}, \Pi^{(y)}$ may be replaced by any topologically equivalent pair of operators, i.e., we may deform or blur the boundaries of the corresponding half-planes.

In the translationally invariant case, the right-hand side of Eq. (128) can be calculated by widening the fuzzy boundaries so that they turn into linear functions. Thus

$$
\nu(P)=2 \pi i \operatorname{tr}(P[[X, P],[Y, P]])
$$

where $X$ and $Y$ are the operators of $x$ - and $y$-coordinate (resp.), and $\operatorname{tr}$ is the trace per unit cell. Passing to the momentum representation and using a two-dimensional analogue of the correspondence (116), we recover Eq. (54).

C.3.3 The integrality of $\nu(P)$. We have seen $\nu(P)$ has topological nature; let us show that it is an integer. Let

$$
Q^{(x)}=P \Pi^{(x)} P, \quad Q^{(y)}=P \Pi^{(y)} P, \quad U^{(x)}=\exp \left(2 \pi i Q^{(y)}\right), \quad U^{(y)}=\exp \left(-2 \pi i Q^{(x)}\right) .
$$

The operator $U^{(x)}$ coincides with the identity matrix away from the $x$-axis, and $U^{(y)}$ is equal to the identity away from the $y$-axis. We may proceed in two different ways.

First proof. Let us regard the two-dimensional lattice as one-dimensional by identifying all sites in each vertical row. Then $U^{(x)}$ satisfies the hypothesis of Theorem C.1 hence it has an integer flow. But the flow can be expressed using Eq. (114) with $Q=Q^{(x)}$. Thus

$$
\begin{aligned}
\mathcal{F}\left(U^{(x)}\right) & =\operatorname{Tr}\left(e^{-2 \pi i Q^{(y)}} Q^{(x)} e^{2 \pi i Q^{(y)}}-Q^{(x)}\right)=\int_{0}^{2 \pi} \operatorname{Tr}\left(\frac{d}{d \varphi}\left(e^{-i \varphi Q^{(y)}} Q^{(x)} e^{i \varphi Q^{(y)}}\right)\right) d \varphi \\
& =2 \pi i \operatorname{Tr}\left[Q^{(x)}, Q^{(y)}\right]=\nu(P) .
\end{aligned}
$$

Note an analogy with the Laughlin argument: the integration over $\varphi$ corresponds to the adiabatic insertion of a magnetic flux quantum.

Second proof (sketch). Let us employ the notion of Fredholm determinant. It is a generalization of the determinant to infinite matrices that are close to the identity, specifically of the form $1+K$, where $K$ has a well-defined trace: ${ }^{14}$

$$
\operatorname{det}(1+K) \stackrel{\text { def }}{=} \exp (\operatorname{Tr} \ln (1+K))=1+\operatorname{Tr} K+\left(-\frac{1}{2} \operatorname{Tr} K^{2}+\frac{1}{2}(\operatorname{Tr} K)^{2}\right)+\ldots
$$

The exact meaning of this definition is as follows: we first obtain a formal expression as a power series in $K$ and then evaluate each term. (If $K$ is a matrix of size $n$, then all terms of degree higher than $n$ vanish.) We claim that

$$
\operatorname{det}\left(U^{(x)} U^{(y)}\left(U^{(x)}\right)^{-1}\left(U^{(y)}\right)^{-1}\right)=1
$$

Indeed, the identity $\operatorname{det}\left(A B A^{-1} B^{-1}\right)=1$ is true at the formal power series level, assuming that the cyclic property of the trace holds for any product of $A-1, A^{-1}-1, B-1, B^{-1}-1$

\footnotetext{
${ }^{14}$ It is important that any matrix product that occurs in the problem has a trace satisfying the cyclic property.
} 
containing at least one factor with $A$ and at least one factor with $B$. On the other hand, one can show that

$$
\operatorname{det}\left(e^{i \varphi_{y} Q^{(y)}} e^{i \varphi_{x} Q^{(x)}} e^{-i \varphi_{y} Q^{(y)}} e^{-i \varphi_{x} Q^{(x)}}\right)=\exp \left(\varphi_{x} \varphi_{y} \operatorname{Tr}\left[Q^{(x)}, Q^{(y)}\right]\right) .
$$

\section{C.4 Majorana numbers}

In this section we study real skew-symmetric matrices satisfying the equation $B^{2}=-1$. Recall that such a matrix defines the ground state $|\Psi\rangle$ of a Majorana system with a quadratic Hamiltonian (cf. Eqs. (26) and (27)). First, we consider finite or general infinite matrices (i.e., work in dimension zero), then proceed to quasidiagonal matrices in dimension one, and finally to dimension two.

C.4.1 Dimension zero: fermionic parity and the Pfaffian. A few more words about the Majorana system, then we will turn to matrices. Let us group the sites (or the corresponding Majorana operators) into pairs $(2 k-1,2 k)$ to form "full" fermionic modes with the occupation number operators $a_{k}^{\dagger} a_{k}=\frac{1}{2}\left(1+i c_{2 k-1} c_{2 k}\right)$. Note that the operator $-i c_{2 k-1} c_{2 k}$ has eigenvalue 1 if the mode is empty and -1 if it is occupied. Thus the total fermionic parity is characterized by the operator $\prod_{k}\left(-i c_{2 k-1} c_{2 k}\right)$. The fermionic parity of the ground state is equal to the Pfaffian of the matrix $B$ :

$$
\prod_{k}\left(-i c_{2 k-1} c_{2 k}\right)|\Psi\rangle=(\operatorname{Pf} B)|\Psi\rangle, \quad \operatorname{Pf} B= \pm 1
$$

Let us recall some standard definitions. The Pfaffian of a skew-symmetric matrix is expressed as a sum over all partitions of the set $\{1, \ldots, 2 N\}$ into pairs, or over all elements of the permutation group $S_{2 N}$ :

$$
\operatorname{Pf} A=\frac{1}{2^{N} N !} \sum_{\tau \in S_{2 N}} \operatorname{sgn}(\tau) A_{\tau(1), \tau(2)} \cdots A_{\tau(2 N-1), \tau(2 N)} .
$$

For example,

$$
\operatorname{Pf}\left(\begin{array}{cc}
0 & a_{12} \\
-a_{12} & 0
\end{array}\right)=a_{12}, \quad \operatorname{Pf}\left(\begin{array}{cccc}
0 & a_{12} & a_{13} & a_{14} \\
-a_{12} & 0 & a_{23} & a_{24} \\
-a_{13} & -a_{23} & 0 & a_{34} \\
-a_{14} & -a_{24} & -a_{34} & 0
\end{array}\right)=a_{12} a_{34}+a_{14} a_{23}-a_{13} a_{24} .
$$

The Pfaffian satisfies the equations

$$
(\operatorname{Pf} A)^{2}=\operatorname{det} A, \quad \operatorname{Pf}\left(W A W^{T}\right)=\operatorname{Pf}(A) \operatorname{det}(W) .
$$

We will also need a generalization of the Pfaffian to infinite matrices, which is called Fredholm Pfaffian [75. Let $A$ and $B$ be real skew-symmetric, $B$ invertible, and $A-B$ have a well-defined trace. Then

$$
\begin{aligned}
\operatorname{Pf}(A, B) \stackrel{\text { def }}{=} \sqrt{\operatorname{det}(1+K)}=\exp \left(\frac{1}{2} \operatorname{Tr} \ln (1+K)\right) \\
\\
=1+\frac{1}{2} \operatorname{Tr} K+\left(-\frac{1}{4} \operatorname{Tr} K^{2}+\frac{1}{8}(\operatorname{Tr} K)^{2}\right)+\ldots, \quad \text { where } K=A B^{-1}-1 .
\end{aligned}
$$


This definition is understood as that of the Fredholm determinant: we first obtain a formal expression as a power series in $K$ and then evaluate each term. (For finite matrices of size $2 N$ we get $\operatorname{Pf}(A, B)=(\operatorname{Pf} A)(\operatorname{Pf} B)^{-1}$; note that the terms of degree higher than $N$ vanish). The Fredholm Pfaffian has the following properties:

$$
\begin{gathered}
\operatorname{Pf}(B, B)=1, \quad \operatorname{Pf}(A, B) \operatorname{Pf}(B, C)=\operatorname{Pf}(A, C), \\
\operatorname{Pf}(A, B)^{2}=\operatorname{det}\left(A B^{-1}\right), \quad \operatorname{Pf}\left(W A W^{T}, V B V^{T}\right)=\operatorname{Pf}(A, B) \operatorname{det}\left(V^{-1} W\right) ; \\
\text { if } A^{2}=B^{2}=-1, \quad \text { then } \operatorname{Pf}(A, B)= \pm 1 .
\end{gathered}
$$

C.4.2 Dimension one: cutting obstruction and the relative Majorana number. Now, consider a matrix that describes the ground state of Majorana fermions on an infinite chain. From a formal point of view, it suffices to say that $B=\left(B_{j k}: j, k \in \mathbb{Z}\right)$ is quasidiagonal, real skew-symmetric, and that $B^{2}=-1$. Suppose that we cut the chain between sites $\eta-1$ and $\eta$. Is it possible to find a new matrix $B^{\prime}$ that contains no cross elements (i.e., has the form $\left.B_{--}^{\prime} \oplus B_{++}^{\prime}\right)$, satisfies the condition $\left(B^{\prime}\right)^{2}=-1$, and coincides with $B$ far away from the cut? The following quantity represents an obstruction for the construction of such a matrix:

$$
\mathcal{M}_{\eta}(B)=\operatorname{Pf}(V(\eta) B V(\eta), B)= \pm 1, \quad \text { where } V(\eta)=1-2 \Pi^{[\eta, \infty)} .
$$

Recall that $\Pi^{[\eta, \infty)}$ denotes the projector onto sites $\eta$ and higher; $(V(\eta) B V(\eta))_{j k}= \pm B_{j k}$, where the minus sign occurs when $j$ and $k$ lie on different sides of the cut.

We claim that the conditions on $B^{\prime}$ cannot be met if $\mathcal{M}_{\eta}(B)=-1$. Indeed,

$$
\begin{aligned}
\mathcal{M}_{\gamma}(B) \mathcal{M}_{\eta}(B)^{-1} & =\operatorname{Pf}(V(\gamma) B V(\gamma), V(\eta) B V(\eta)) \\
& =\operatorname{det}\left(V(\eta)^{-1} V(\gamma)\right)=(-1)^{\gamma-\eta} .
\end{aligned}
$$

The same is true for $B^{\prime}$, hence the relative Majorana number,

$$
\mathcal{M}\left(B, B^{\prime}\right) \stackrel{\text { def }}{=} \mathcal{M}_{\gamma}(B) \mathcal{M}_{\gamma}\left(B^{\prime}\right)
$$

does not depend on $\gamma$. However, $\mathcal{M}_{\gamma}\left(B^{\prime}\right)=\mathcal{M}_{\gamma}(B)$ if $|\gamma-\eta|$ is large enough (since $B$ and $B^{\prime}$ agree far away from the cut), while $\mathcal{M}_{\eta}\left(B^{\prime}\right)=1$ (because $B^{\prime}=B_{--}^{\prime} \oplus B_{++}^{\prime}$ ) and $\mathcal{M}_{\eta}(B)=-1$ (by assumption). We have arrived at a contradiction.

From the physical point of view, the impossibility to construct a suitable matrix $B^{\prime}$ indicates the presence of unpaired Majorana modes on both sides of the cut. This situation may be described by matrices $B_{--}^{\prime}$ and $B_{++}^{\prime}$ having one zero eigenvalue.

The cutting obstruction and the relative Majorana number can also be expressed as Fredholm determinants. To this end, consider the quasidiagonal matrix

$$
W=V(0) \exp (\pi X), \quad \text { where } X=\frac{1}{2}\left(\Pi^{(x)} B+B \Pi^{(x)}\right), \quad \Pi^{(x)} \stackrel{\text { def }}{=} \Pi^{[0, \infty)} .
$$

Clearly, $X$ is real skew-symmetric and commutes with $B$. Hence $W$ is orthogonal, and $W B W^{T}=$ $V(0) B V(0)$. On the other hand, $X$ is equal to $B$ at $+\infty$ and vanishes at $-\infty$, therefore $W$ is equal to 1 at $\pm \infty$. One can actually show that $W-1$ has a well-defined trace. It follows that

$$
\mathcal{M}_{0}(B)=\operatorname{Pf}\left(W B W^{T}, B\right)=\operatorname{det} W
$$

Similarly, one can define $X^{\prime}, W^{\prime}$ as functions of $B^{\prime}$. Thus

$$
\mathcal{M}\left(B, B^{\prime}\right)=\operatorname{det}\left(W^{-1} W^{\prime}\right)=\operatorname{det}\left(e^{-\pi X} e^{\pi X^{\prime}}\right) .
$$


C.4.3 Applications to two dimensions. Eq. (147) may be used as a more general definition of the relative Majorana number, which works even if $\mathcal{M}_{\eta}(B)$ and $\mathcal{M}_{\eta}\left(B^{\prime}\right)$ do not exist. For example, let $B$ be a quasidiagonal matrix in two dimensions and $B^{\prime}=\left(1-2 \Pi^{(y)}\right) B\left(1-2 \Pi^{(y)}\right)$. In other words, $B_{j k}^{\prime}= \pm B_{j k}$, where the minus sign occurs when $j$ and $k$ lie on different sides of the $x$-axis. If we regard the system as one-dimensional (collapsing it in the $y$ direction), the Majorana number $\mathcal{M}\left(B, B^{\prime}\right)$ is well-defined and can be expressed in terms of the projector $P=\frac{1}{2}(1-i B)$. The calculation follows.

First, we obtain an expression for the operator $X$ :

$$
X=i\left(P \Pi^{(x)} P-(1-P) \Pi^{(x)}(1-P)\right) .
$$

Then we use the relation $1-2 \Pi^{(y)}=\exp \left( \pm i \pi \Pi^{(y)}\right)$ :

$$
\mathcal{M}\left(B, B^{\prime}\right)=\operatorname{det}\left(e^{-\pi X} e^{-i \pi \Pi^{(y)}} e^{\pi X} e^{i \pi \Pi^{(y)}}\right)=\exp \left(i \pi^{2} \operatorname{Tr}\left[X, \Pi^{(y)}\right]\right) .
$$

Finally, we compute the trace of the commutator:

$$
\begin{aligned}
\operatorname{Tr}\left[X, \Pi^{(y)}\right] & =\operatorname{Tr}\left(\left(P^{2}+(1-P)^{2}\right)\left[X, \Pi^{(y)}\right]\right)=\operatorname{Tr}\left(P\left[X, \Pi^{(y)}\right] P+(1-P)\left[X, \Pi^{(y)}\right](1-P)\right) \\
& =i \operatorname{Tr}\left[P \Pi^{(x)} P, P \Pi^{(y)} P\right]-i \operatorname{Tr}\left[(1-P) \Pi^{(x)}(1-P),(1-P) \Pi^{(y)}(1-P)\right] \\
& =\frac{1}{2 \pi}(\nu(P)-\nu(1-P))=\frac{1}{\pi} \nu(P),
\end{aligned}
$$

because $\nu(1-P)=-\nu(P)$. Thus

$$
\mathcal{M}\left(B, B^{\prime}\right)=(-1)^{\nu(P)} .
$$

Equation (148) can be applied to the geometry shown in Fig. 15. In this case, $B$ and $B^{\prime}$ correspond to two different $\mathbb{Z}_{2}$-field configurations: the first is regarded as vortex-free and the second has a vortex at the origin. The specific assumptions are as follows: (i) $B$ and $B^{\prime}$ are real skew-symmetric quasidiagonal matrices in two dimensions; (ii) $B$ and $B^{\prime}$ coincide, except that $B_{j k}^{\prime}=-B_{j k}$ for those links which cross the ray $r$; (iii) $B^{2}=-1$ (therefore $\left(B^{\prime}\right)^{2}=-1$ far away from the vortex). Note that $\mathcal{M}_{\eta}(B)$ and $\mathcal{M}_{\eta}\left(B^{\prime}\right)$ may be defined for a sufficiently large loop $\eta$ enclosing the vortex. Let $|\eta|$ be the number of sites inside the loop. Then the absolute Majorana numbers $\mathcal{M}(B) \stackrel{\text { def }}{=}(-1)^{|\eta|} \mathcal{M}_{\eta}(B)$ and $\mathcal{M}\left(B^{\prime}\right) \stackrel{\text { def }}{=}(-1)^{|\eta|} \mathcal{M}_{\eta}\left(B^{\prime}\right)$ do not depend on $\eta$ and indicate the presence of unpaired Majorana modes in $B$ and $B^{\prime}$, respectively. However, an unpaired mode cannot exist in $B$ since $B^{2}=-1$; therefore $\mathcal{M}\left(B, B^{\prime}\right)=\mathcal{M}\left(B^{\prime}\right)$. We conclude that

$$
\mathcal{M}\left(B^{\prime}\right)=(-1)^{\nu(P)} .
$$

\section{Appendix D: Some remarks on the chiral central charge}

This appendix is an attempt to understand the physical and mathematical meaning of the chiral central charge beyond the CFT framework. Recall that chiral central charge is just the coefficient $c_{-}$in the edge energy current formula (57). This definition will be refined in Sec. D.1. 
It turns out that the edge energy current is a property of the bulk ground state. The edge current is related to a bulk 2-current. Although the theory is in its embryonic stage, it looks like a second-quantized version of the Chern number for quasidiagonal matrices (cf. Sec. C.3).

In Sec. D.2 we discuss modular transformations of the partition function on a space-time torus. In particular, the phase factor $e^{-2 \pi i c_{-} / 24}$ appears in the description of the Dehn twist along a time circle. However, other modular transformations are difficult to define because space and time are not physically equivalent.

\section{D.1 The edge energy current and a bulk 2-current}

D.1.1 Energy current in the Hamiltonian formalism. Let us represent the Hamiltonian as a sum of local terms:

$$
H=\sum_{j} H_{j}
$$

where $H_{j}$ is a Hermitian operator acting only on spins in some neighborhood of the point $j$. Note that the decomposition into local terms is not unique. We also assume that the ground state $|\Psi\rangle$ is separated from excited states by an energy gap; thus equal-time spin correlators decay exponentially with distance [16. We may slightly extend the notion of locality so that $H_{j}$ acts on all spins but

$$
\left\|\left[H_{j}, \sigma_{k}^{\alpha}\right]\right\| \leqslant u(|j-k|),
$$

where $u$ is some function with fast decay at infinity (faster than any power). Then the equaltime correlators also decay faster than any power, provided the spectrum is gapped.

Microscopic energy current can be defined for any system with Hamiltonian (150) at finite temperature. It is convenient to fix the temperature at $T=1$ and vary the Hamiltonian instead. The thermal average of an operator $X$ is defined in the standard way:

$$
\langle X\rangle=\operatorname{Tr}(\rho X), \quad \text { where } \rho=Z^{-1} e^{-H}, \quad Z=\operatorname{Tr} e^{-H} .
$$

According to the Heisenberg equation,

$$
\frac{d H_{j}}{d t}=-i\left[H_{j}, H\right]=\sum_{k} \hat{f}_{j k}, \quad \text { where } \quad \hat{f}_{j k}=-i\left[H_{j}, H_{k}\right] .
$$

Thus the operator $\hat{f}_{j k}$ characterizes the energy current from $k$ to $j$. We denote its thermal average by $f_{j k}$ :

$$
f_{j k}=f\left(H_{j}, H_{k}\right), \quad \text { where } \quad f(A, B) \stackrel{\text { def }}{=}\langle-i[A, B]\rangle .
$$

(Note that this definition depends on the decomposition of the Hamiltonian into local terms.) Of course, the energy current is conserved:

$$
(\partial f)_{k} \stackrel{\text { def }}{=} \sum_{j} f_{j k}=0 .
$$

Indeed,

$$
(\partial f)_{k}=f\left(H, H_{k}\right)=\operatorname{Tr}\left(-i \rho\left[H, H_{k}\right]\right)=\operatorname{Tr}\left(-i[\rho, H] H_{k}\right)=0,
$$

since $\rho$ and $H$ commute. 
Let us try to apply the general formula (154) to a specific geometry. Let $H^{(\infty)}$ be the Hamiltonian of an infinite two-dimensional system with a ground state $|\Psi\rangle$ and gapped excitations. To simulate an edge, we introduce another Hamiltonian,

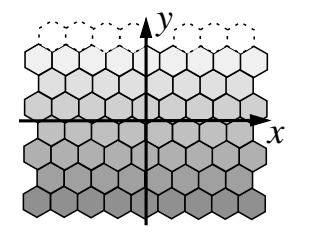

$$
H=\sum_{j} H_{j}, \quad H_{j}=\left\{\begin{array}{cl}
0 & \text { if } y(j) \rightarrow+\infty \\
\beta_{j} H_{j}^{(\infty)}, \beta_{j} \rightarrow \infty & \text { if } y(j) \rightarrow-\infty
\end{array}\right.
$$

where $y(j)$ is the $y$-coordinate of the point $j$. Thus the lower part of the system is "bulk material" characterized by the state $|\Psi\rangle\langle\Psi|$, whereas the upper part is "empty space" (actually, a set of noninteracting spins in the maximally mixed state). By definition, the chiral central charge is $12 / \pi$ times the total energy current in the negative $x$-direction.

D.1.2 Representing the ground state by local constraints. At this point, we have encountered a technical difficulty: the energy current does not necessarily vanish in the bulk. The problem can be avoided if we describe the ground state by a set of local constraints. (A local constraint is a local operator that annihilates the ground state.) Hamiltonians that are explicitly written as sums of local constraints, e.g., the Rokhsar-Kivelson model [76, 19], have provided a lot of insight into properties of quantum many-body systems.

Proposition D.1. Any gapped local Hamiltonian $H=\sum_{j} H_{j}$ whose ground state $|\Psi\rangle$ has zero energy can be represented as a sum of Hermitian local operators $\widetilde{H}_{j}$ such that $\widetilde{H}_{j}|\Psi\rangle=0$.

Proof sketch. Without loss of generality we may assume that $\left\langle\Psi\left|H_{j}\right| \Psi\right\rangle=0$. (If not, change $H_{j}$ by a constant.) Let all excited states have energy greater or equal to $\Delta$. Choose a smooth function $\widehat{w}(\varepsilon)$ such that

$$
\widehat{w}(-\varepsilon)=\widehat{w}(\varepsilon)^{*}, \quad \widehat{w}(0)=1, \quad \widehat{w}(\varepsilon)=0 \text { for }|\varepsilon| \geqslant \Delta .
$$

Its Fourier transform, $w(t)=\int \widehat{w}(\varepsilon) e^{-\varepsilon t} \frac{d \varepsilon}{2 \pi}$ decays faster than any power of $t$ though more slowly than $e^{-\gamma|t|}$ (because $\widehat{w}$ is smooth but not analytic). We set

$$
\widetilde{H}_{j}=\int_{-\infty}^{\infty} e^{i H t} H_{j} e^{-i H t} w(t) d t
$$

It is easy to check that $\sum_{j} \widetilde{H}_{j}=H$ and $\widetilde{H}_{j}|\Psi\rangle=0$. A locality condition of the form (151) can be established using the bound of Lieb and Robinson [77] on correlation propagation (cf. [16]).

For our purposes, we can actually use an arbitrary gapped Hamiltonian $X=\sum_{j} X_{j}$ made up of local constraints for the given state $|\Psi\rangle$. It turns out that the chiral central charge depends only on the ground state. Indeed, one can interpolate between different sets of local constraints for the same state. Consider the situation where the constrains are $X_{j}$ in close proximity of the edge, gradually changing to $X_{j}^{\prime}$ deeper in the bulk. No energy current is associated with the transition between $X$ and $X^{\prime}$.

As an aside, we conjecture that there is a set of local constraints $Y_{j}$ such that the Hamiltonian $Y=\sum_{j} Y_{j}^{\dagger} Y_{j}$ is gapped. Thus excitations can be efficiently detected locally, by coupling each 
$Y_{j}$ to an external indicator: $H_{\text {detector }}=\sum_{j}\left(Y_{j} \otimes(|1\rangle\langle 0|)_{j}+Y_{j}^{\dagger} \otimes(|0\rangle\langle 1|)_{j}\right)$. A stronger version of this conjecture asserts that the original Hamiltonian $H$ can be represented as $\sum_{j} Y_{j}^{\dagger} Y_{j}$, where $Y_{j}$ are local constraints. This is true for the quadratic fermionic Hamiltonian (18), which needs to be offset by its smallest eigenvalue (25) so that the ground state energy becomes zero. It is easy to see that the matrix $|i A|$ is symmetric and that $\sum_{j, k}(|i A|)_{j k} c_{j} c_{k}$ is equal to $\operatorname{Tr}|i A|$ times the identity operator. Hence

$$
H=\frac{1}{4} \sum_{j, k}\left(i A_{j k}+(|i A|)_{j k}\right) c_{j} c_{k}=\sum_{j, k} D_{j k} c_{j} c_{k}, \quad \text { where } D=\frac{1}{4}(i A+|i A|) \geqslant 0
$$

(meaning that $D$ is positive semidefinite). The matrix $\sqrt{D}$ is quasidiagonal, provided $A$ is quasidiagonal and has a spectral gap around zero. Thus we can represent the Hamiltonian in the desired form:

$$
H=\sum_{m} Y_{m}^{\dagger} Y_{m}, \quad \text { where } \quad Y_{m}=\sum_{k}(\sqrt{D})_{m k} c_{k} .
$$

D.1.3 Bulk 2-current. In the disk geometry (i.e., when interaction is strong near the origin and vanishes at infinity), the chiral central charge is $12 / \pi$ times the energy current in the counterclockwise direction. Let us divide the disk into three sectors, $A, B$, and $C$ in the counterclockwise order (see picture in Eq. (124), or in Eq. (160) below). In this setting, the energy current is $\sum_{k \in B} \sum_{l \in A} f_{k l}$.

Let us try to represent the chiral central charge $c_{-}$in a form that would explicitly depend on the bulk Hamiltonian rather than the edge. To this end, we construct a 2-current $h$ such that

$$
f=\partial h, \quad \text { where } \quad(\partial h)_{k l} \stackrel{\text { def }}{=} \sum_{j} h_{j k l} .
$$

(A 2-current is an antisymmetric function of three lattice sites that decays fast enough as the distance between any two sites increases.) Then in the three-sector geometry we have:

$$
\sum_{k \in B} \sum_{l \in A} f_{k l}=\sum_{k \in B} \sum_{l \in A}\left(\sum_{j} h_{j k l}\right)=-\sum_{j \in A} \sum_{k \in B} \sum_{l \in C} h_{j k l} .
$$

Thus,

$$
\text { :a: } \quad c_{-}=-\frac{12}{\pi} \sum_{j \in A} \sum_{k \in B} \sum_{l \in C} h_{j k l} .
$$

The last sum is dominated by a neighborhood of the triple contact point between $A, B$, and $C$.

Unfortunately, there is no canonical expression for $h_{j k l}$. Instead, we can define a canonical object $g$ which is a 2-current on the lattice and a 1-form on the space of local Hamiltonians. It satisfies the condition

$$
\partial g=d f
$$

A suitable 2-current $h$ is obtained by integrating $g$ over an arbitrary path $H(\beta): \beta \in[0, \infty)$ in the space of spin Hamiltonians. Here we assume that $H(\beta)$ is a sum of local terms $H_{j}(\beta)$, the corresponding thermal state has short-range correlators, $H(0)=0$, and $H(\beta) \approx \beta H^{(\infty)}$ as $\beta \rightarrow \infty$. Thus we implicitly accept the following conjecture. 
Conjecture D.2. The ground state of a gapped local Hamiltonian on a two-dimensional lattice can be transformed into the maximally mixed state without a phase transition.

(In this formulation, $\beta$ changes from $\infty$ to 0 .) Note that usual symmetry-related phase transitions are not a problem because $H(\beta)$ is not required to be symmetric. Rather, we should worry about a transition from a topological phase to a non-topological one. However, in two dimensions the long-range topological order in the ground state is destroyed at any finite temperature. Indeed, all topological excitations are point-like and not confined, therefore they have a finite density at any non-zero temperature. This argument does not apply to threedimensional systems, where string-like excitations are possible.

Let us give an explicit formula for $g$. For any two operators $A$ and $B$, we define the Matsubara time-ordered average, as well as its "truncated" version: ${ }^{15}$

$$
\begin{aligned}
& \langle A(\tau) B(0)\rangle=Z^{-1} \operatorname{Tr}\left(e^{-(1-\tau) H} A e^{-\tau H} B\right), \quad \text { where } \quad Z=\operatorname{Tr} e^{-H}, \quad 0 \leqslant \tau \leqslant 1 ; \\
& \langle\langle A(\tau) B(0)\rangle\rangle=\langle A(\tau) B(0)\rangle-\langle A\rangle\langle B\rangle .
\end{aligned}
$$

Next, we define a function of three operators:

$$
\mu(A, B, C)=i \int_{0}^{1}\langle\langle A(\tau)[B, C](0)\rangle\rangle d \tau .
$$

Finally, we assume that the Hamiltonian $H$ and the operators $A, B, C$ depend on some parameters, so that we can differentiate them. Now we can define $g$ :

$$
g(A, B, C)=\mu(d A, B, C)+\mu(d B, C, A)+\mu(d C, A, B), \quad g_{j k l}=g\left(H_{j}, H_{k}, H_{l}\right) .
$$

Let us verify Eq. (161). Note that $\mu(X, Y, H)=-i\langle[X, Y]\rangle$ because $\langle X(\tau)[Y, H](0)\rangle=$ $\frac{d}{d \tau}\langle X(\tau) Y(0)\rangle$ and $\langle[Y, H]\rangle=0$. We proceed as follows:

$$
\begin{aligned}
\partial g(B, C) & =g(H, B, C)=\mu(d H, B, C)+\mu(d B, C, H)+\mu(d C, H, B) \\
& =\left(-i Z^{-1} \operatorname{Tr}\left(d e^{-H}[B, C]\right)-i\langle d H\rangle\langle[B, C]\rangle\right)-i\langle[d B, C]\rangle+i\langle[d C, B]\rangle \\
& =d\left(-i Z^{-1} \operatorname{Tr}\left(e^{-H}[B, C]\right)\right)=d f(B, C) .
\end{aligned}
$$

\section{D.2 Modular transformations beyond CFT}

The modular invariance 62] is usually formulated in the CFT framework. In this section, we demonstrate some modular properties of the partition function of edge excitations, not assuming conformal, rotational, or any other symmetry. First, let us generalize the transformation of the vacuum character ${ }^{16}$ under the Dehn twist:

$$
\chi_{1}(w+1)=e^{-2 \pi i c_{-} / 24} \chi_{1}(w),
$$

\footnotetext{
${ }^{15}$ This notation is reminiscent of the formula $A(\tau)=e^{\tau H} A e^{-\tau H}$, but the latter may be problematic to use because $e^{\tau H} \approx \exp \left(\tau \beta H^{(\infty)}\right)$ diverges as $\beta \rightarrow \infty$. Thus the expression $\langle A(\tau) B(0)\rangle$ should be viewed as a whole.

${ }^{16}$ Some care should be taken to make a proper connection between the physics of edge modes and the the CFT formalism. If all edge modes have the same chirality, characters correspond to representations of some chiral algebra (as is usual in CFT). In general, the vacuum character $\chi_{1}$ is defined as a sum over all fields with trivial monodromy whereas $\chi_{a}$ includes all fields that occur in the two-dimensional theory when an anyon of type $a$ emerges on the surface (see Fig. 9p on page 29).
} 
where $w$ is the modulus of the torus and the subscript 1 refers to the vacuum sector.

In fact, the derivation of Eq. (166) does not require much more than the knowledge of the energy-momentum tensor, or just its $\tau x$-component, which is exactly the energy current $I$. (We use imaginary time, $\tau=i t$.) In the thermodinamic limit, the coordinate transformation ${ }^{17}$ $(x, \tau) \rightarrow(x, \tau+\xi(x))$ induces the action change $\delta S=i I \int \frac{d \xi}{d x} d x d \tau$. If the imaginary time is cyclic, then twisting it by a full circle results in the action change $\delta S=i \frac{\pi}{12} c_{-}$and the multiplication of the partition function by $e^{-S}=e^{-2 \pi i c_{-} / 24}$. We now describe a particular setting in which this result is applicable.

Let us consider the partition function $Z=\operatorname{Tr} e^{-H / T}$ of a spin system on a disk. As is usual, we think of the evolution over the time period $1 / T$, making the edge into a torus and the disk itself into the three-dimensional manifold $M=D^{2} \times S^{1}$. Let us suppose that the temperature $T$ is much smaller than the energy gap in the bulk, so that $Z$ is mostly determined by edge excitations. In fact, the partition function depends on full detail of the edge Hamiltonian as well as some conditions in the bulk. For example, we may place an anyon of type $a$ somewhere in the disk, which slightly changes the edge excitation spectrum. In other words, we insert a particle worldline $\ell$ into the manifold $M$, or act by the time-like Wilson loop operator $W_{a}(\ell)$. Thus a partition function $Z_{a}$ is defined. If all edge modes propagate with the same velocity $v$ and can be described by a CFT, then $Z_{a}=\chi_{a}(-1 / w)$, where $w=i L T / v$. In the limit $w \rightarrow \infty$,

$$
Z_{a}=\chi_{a}(-1 / w)=\sum_{b} \mathcal{S}_{a b} \chi_{b}(w) \approx \mathcal{S}_{a 1} \chi_{1}(w)
$$

because all characters $\chi_{b}(w)$ for $b \neq 1$ are exponentially smaller than the vacuum character $\chi_{1}(w)$.

The following argument does not depend on CFT. As already mentioned, twisting the torus by $\xi$ along a time circle leads to the the action change by $i I \xi / T$, or the multiplication of the partition function by $e^{-i I \xi / T}$. (The sign in the exponent depends on the direction of the twist; we choose it to be consistent with the CFT formula.) For the full twist, $\xi=1 / T$, we get this equation:

$$
Z_{a}^{\prime} \approx e^{-2 \pi i c_{-} / 24} Z_{a}
$$

Note that the finite-size quantization of edge modes might affect the energy current. We therefore assume that the disk circumference $L$ is much larger than the edge correlation length $l_{\text {edge }} \sim v / T$, where $v$ is the maximum group velocity of any excitation in the system. ${ }^{18}$ Small corrections proportional to $\exp \left(-L / l_{\text {edge }}\right)$ are expected. Thus, Eq. (168) and its estimated precision are in perfect agreement with the CFT formulas (166) and (167).

With some more work, one can also derive an analogue of this CFT result:

$$
\chi_{b}(w+1)=e^{-2 \pi i c_{-} / 24} \theta_{b} \chi_{b}(w),
$$

\footnotetext{
${ }^{17}$ For lattice models, one should consider the transformation $(j, \tau) \rightarrow\left(j, \tau+\xi_{j}\right)$. It should be possible to give an exact quantum-mechanical interpretation of such a reparametrization by coupling the discrete derivative $\eta_{j k}=\xi_{j}-\xi_{k}$ to the energy current operator $\hat{f}_{j k}=-i\left[H_{j}, H_{k}\right]$, where $H_{j}, H_{k}$ are local terms in the Hamiltonian. Such coupling is unambiguously defined only in the first order in $\eta_{j k}$, but that does not affect the thermodynamic limit.

${ }^{18} \mathrm{~A}$ rigorous and completely general upper bound for the group velocity was obtained by Lieb and Robinson [77]. It is consistent with the rough estimate $v \lesssim r J$, where $J$ is the inter-spin interaction strength and $r$ is its range.
} 
where $\theta_{b}$ is the topological spin of $b$. In the non-conformal setting, the role of the characters $\chi_{b}(w)$ is played by linear combinations of the partition functions:

$$
\widetilde{Z}_{b}=\sum_{a} s_{b \bar{a}} Z_{a}
$$

where $s_{a b}$ are the entries of a topological $S$-matrix defined in terms of anyonic braiding, see Eq. (223) on page 84. (We distinguish it form the modular matrix $\mathcal{S}$, though they actually coincide when the latter is defined.) A calculation based on the insertion of a space-like Wilson loop $W_{b}(\widetilde{\ell})$ shows that

$$
\widetilde{Z}_{b}^{\prime} \approx e^{-2 \pi i c_{-} / 24} \theta_{b} \widetilde{Z}_{b}
$$

This equation is indeed similar to (169) with one important difference: unlike $\chi_{a}(w)$ and $\chi_{a}(-1 / w)$, the numbers $\widetilde{Z}_{a}$ and $Z_{a}$ are not obtained by evaluating the same function. Another problem is that the Dehn twist can only be performed along a time circle. It is therefore not clear whether the full modular invariance can be established without conformal symmetry, or at least a $90^{\circ}$-rotation symmetry of the space-time manifold.

\section{Appendix E: Algebraic theory of anyons}

This appendix is an attempt to present an existing but difficult and somewhat obscure theory in an accessible form, especially for the reader without extensive field theory knowledge. (But we do assume some mathematical background and the willingness to follow abstract arguments.) The presently available resources may be divided into three categories: the original field-theoretic papers where the relevant mathematical structure was discovered [4, 5], local field theory theory papers [6, 7] (see Haag's book [8] for a general reference on this subject), and purely mathematical expositions [68, 79, 80. Unfortunately, all these texts have different focus and/or difficult to read. A nice elementary introduction can be found in Preskill's lecture notes on quantum computation [81, but more detail is needed for our purposes.

The theory described below is applicable to two-dimensional many-body systems with shortrange interactions and an energy gap. Using some intuition about local excitations in such systems, we characterize the properties of anyons by a set of axioms and derive some corollaries. This approach is somewhat similar to what has been done rigorously in local field theory. However, we avoid many difficulties by keeping the discussion at the physical level until all essential properties are cast into a finite algebraic form, at which point we enter the realm of mathematics.

More specifically, we claim that finite-energy excitations are classified by superselection sectors: each sector consists of states that can be transformed one to another by local operations. (We do not care about rigorous definition here.) It is assumed that the full classification can be established by considering a small neighborhood of an arbitrary point (of size compared to the correlation length). Another assumption is that local excitations can be moved from one place to another by applying an operator along an arbitrary path. Note that the quasiparticle transport need not be adiabatic, nor we require translational symmetry. Topological properties, such as braiding rules, have invariant meaning independent of possible disorder, cf. Fig. 10 


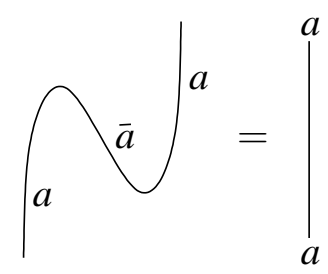

a)

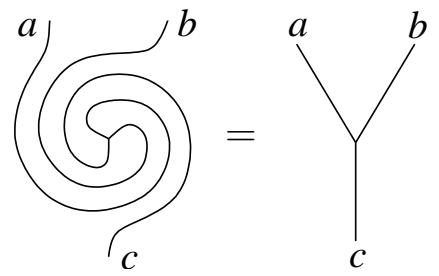

b)

Figure 16: Some "obvious" identities.

on page 34. Using some additional arguments, we will arrive at a theory that may be called unitary braided fusion category (UBFC) (= unitary ribbon category) in abstract language. ${ }^{19}$

With this approach, we get a weaker set of axioms compared to an analogous algebraic structure in conformal field theory. Therefore some "obvious" properties require a proof. (A similar situation occurs in local field theory, but our exposition is different in some details and hopefully simpler.) For nonrelativistic quantum-mechanical models, the space and time are not equivalent even if we use the imaginary time formalism. Thus the notion of an antiparticle being a particle propagating back in time must be applied with caution. The identity in Fig. 16] illustrates a standard use of this notion. (Shown in the figure are space-time diagrams; time goes up.) On the left-hand side of the identity a particle $a$ annihilates with its antiparticle $\bar{a}$ that was created as part of an $\bar{a} a$ pair. If space and time were related by a rotational symmetry, then the zigzag on the particle worldline could simply be removed. In our formalism, this equation is satisfied by a suitable choice of normalization factors for the creation and annihilation operators of particle-antiparticle pairs. However, the relation in Fig. 16b is a nontrivial theorem; it follows from the positivity of Hermitian inner product, see Section E.2. (In the categorical language, this result reads: "Any unitary fusion category admits a pivotal structure".)

Having formulated fusion and braiding axioms, we will touch upon another question: What UBFCs can be realized by a Hamiltonian with given symmetry properties? The simplest case is where there is no built-in symmetry or associated conservation laws. That is, we assume that the system consists of spins (or other bosonic degrees of freedom) and the Hamiltonian is in generic position. (Fermions are excluded because their number is always conserved modulo 2.) Thus, any effective conservation law for quasiparticles must have topological nature. In Sec.E.5 we formulate this condition as a certain nondegeneracy property of the braiding rules. Theories satisfying this axiom are called unitary modular categories (UMC). ${ }^{20}$

Unfortunately, UMC does not capture one important robust characteristic of the physical system, namely the chiral central charge $c_{-}$. However, there is a beautiful relation [7, 61, that fixes $c_{-} \bmod 8$ :

$$
\mathcal{D}^{-1} \sum_{a} d_{a}^{2} \theta_{a}=e^{2 \pi i c_{-} / 8}, \quad \text { where } \quad \mathcal{D}=\sqrt{\sum_{a} d_{a}^{2}},
$$

\footnotetext{
${ }^{19}$ Note for experts (the general reader shouldn't worry about this): a fusion category is a $k$-linear semisimple rigid tensor category with finite-dimensional morphism spaces and finitely many simple objects such that $\operatorname{End}(\mathbf{1})=k$, see 30 . We take $k=\mathbb{C}$. A unitary fusion category is automatically pivotal, spherical, and nondegenerate. In the presence of braiding, these properties imply the existence of a ribbon twist.

${ }^{20}$ The term "modular" refers to the possibility to define a Hilbert space associated with a torus, on which the modular group acts. More generally, one can construct a so-called modular functor and a topological quantum field theory 67, 68, 79, (but we do not cover these topics).
} 
$d_{a}$ is the quantum dimension of the superselection sector $a$ (see Sec. E.2) and $\theta_{a}$ is the topological spin (see Sec. E.3). The left-hand side of Eq. (172) is denoted by $\Theta$; its algebraic meaning will be explained in Sec. E.5. Roughly, $\Theta=S^{-1} T S T S T$, where $S$ is a so-called topological $S$-matrix and $T$ is the diagonal matrix with entries $\theta_{a}$. We will see that $\Theta$ is a root of unity, hence Eq. (172) implies that $c_{-}$is rational.

The standard proof of Eq. (172) is based on the assumption that the system admits conformally invariant edge modes under suitable boundary conditions. Then one can employ the modular invariance of the partition function on a space-time torus, which is established in the CFT framework [62]. It is not known whether the conformality hypothesis is true in general. One may, however, hope to find a proof that would not depend on conformal symmetry. In Sec. D.2 we made partial progress toward this goal. Specifically, we showed that the matrix $\mathcal{T}=e^{-2 \pi i c_{-} / 24} T$ corresponds to the Dehn twist along a time circle. While $S$ can also be regarded as a modular transformation in a suitable mathematical theory, it is not clear whether that theory is applicable in the same context as the expression for $\mathcal{T}$.

In the remaining part of the appendix we do not discuss the chiral central charge. Our primary goal is to define a unitary braided fusion category (UBFC) and its special case, unitary modular category (UMC). The definition is rather long, so we break it into several parts. Each part contains some physical motivation, formal axioms in terms of basic data and equations, as well as some corollaries. Note that our formulation of the axioms does not involve the mathematical notion of category, so we call the corresponding objects "theories" (e.g., "fusion theory" instead of "fusion category"). Only later do we introduce the powerful but heavy language of categories and functors (also known as "abstract nonsense"). It is surely overkill for most of the problems considered in this paper, but the concept of tensor functor may actually be useful in the study of phase transitions in anyonic systems, e.g., Bose-condensation of spinless particle and weak symmetry breaking. (The last topic is considered in Appendix [F])

\section{E.1 Fusion theory}

In this section we consider anyons on a line (though the physical system is two-dimensional). The motivation for the definitions has been provided in Sec. 8.4 (esp. in 8.4.2). Each particle is characterized by a superselection sector label (describing its "anyonic charge"). The position and local degrees of freedom may be ignored, but the order of particles is important. The main element of the the fusion theory is the space $V_{c}^{a b}$ — the state space of particles $a$ and $b$ restricted to have total anyonic charge $c$. More exactly, vectors in this space correspond to different ways of splitting $c$ into $a$ and $b$, or equivalence classes of local operators that effect the splitting.

E.1.1 Splitting and fusion operators. While $V_{c}^{a b}$ may be called "splitting space", the corresponding fusion space is denoted by $V_{a b}^{c}$. If $\psi \in V_{c}^{a b}$ is a splitting operator, then $\psi^{\dagger} \in V_{a b}^{c}$ is a fusion operator:

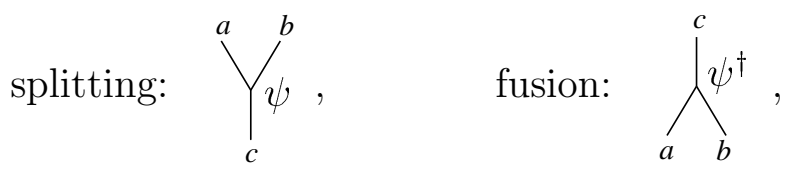

where $\psi$ and $\psi^{\dagger}$ label the corresponding vertices. One may also use Dirac's notation, i.e., write $|\psi\rangle$ instead of $\psi$ and $\langle\psi|$ instead of $\psi^{\dagger}$. By definition, the Hermitian inner product corresponds 
to the operator multiplication. Specifically, if $\xi, \eta \in V_{c}^{a b}$, then

$$
a \bigcap_{c}^{c} \eta^{\dagger} b=\left.\langle\eta \mid \xi\rangle\right|_{c} ^{c}, \quad \text { i.e., } \quad \eta^{\dagger} \xi=\langle\eta \mid \xi\rangle \mathrm{id}_{c}
$$

Similarly, one can define splitting and fusion of multiple particles. The most general process is one transforming particles $b_{1}, \ldots, b_{m}$ into $a_{1}, \ldots, a_{k}$. It can be performed in two steps: first, fusing all the particles into one, and then splitting it as required. Therefore

$$
V_{b_{1} \ldots b_{m}}^{a_{1} \ldots a_{k}}=\bigoplus_{c} V_{c}^{a_{1} \ldots a_{k}} \otimes V_{b_{1} \ldots b_{m}}^{c}
$$

For example, the identity operator acting on particles $a$ and $b$ can be decomposed as follows:

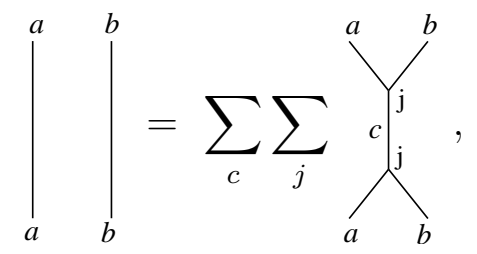

where $j$ stands for both $\psi_{j}$ and $\psi_{j}^{\dagger}$ — basis vectors in $V_{c}^{a b}$ and $V_{a b}^{c}$, respectively.

The description of splitting and fusion will be complete when we understand relations between different spaces $V_{c}^{a_{1} \ldots a_{k}}$. That is the topic of the following subsection.

E.1.2 Basic data and equations. The basic data include a set of superselection sectors, fusion rules, and associativity relations. The latter satisfy so-called pentagon and triangle equations. An additional condition (requiring the nondegeneracy of certain operators) will be formulated in Sec. E.2.

Superselection sectors (also called "particle types", or "labels"): They form a finite set $M$.

Fusion rules: For any combination of $a, b, c \in M$ there is a fixed finite-dimensional Hilbert space $V_{c}^{a b}$. The numbers $N_{a b}^{c}=\operatorname{dim} V_{a b}^{c}=\operatorname{dim} V_{c}^{a b}$ are called fusion multiplicities. The choice of a special element $1 \in M$ (the vacuum sector) is also considered part of the fusion rules.

Associativity relations: For each $a, b, c, d \in M$ there is a canonical unitary isomorphism between two Hilbert spaces:

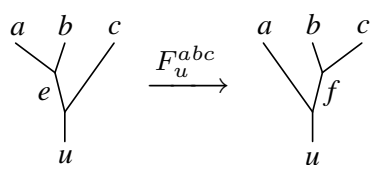

$$
F_{u}^{a b c}: \bigoplus_{e} V_{e}^{a b} \otimes V_{u}^{e c} \rightarrow \bigoplus_{f} V_{u}^{a f} \otimes V_{f}^{b c}
$$

Note that both spaces in question are simply different representation of the same physical space $V_{u}^{a b c}$ — that of the particles $a, b$, and $c$ restricted to have total anyonic charge $u$. Therefore one may regard the isomorphism $F_{u}^{a b c}$ as equality. This view is formally justified by the pentagon 


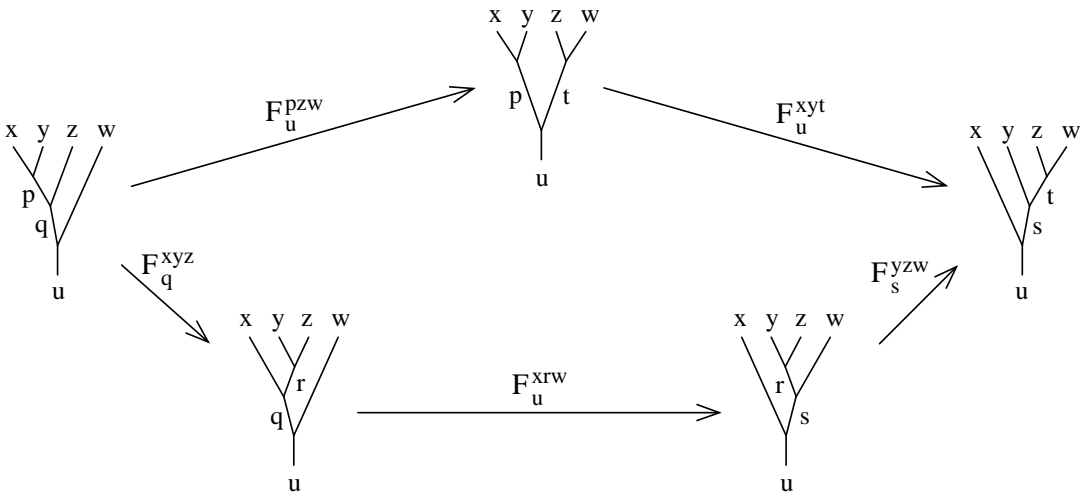

a)

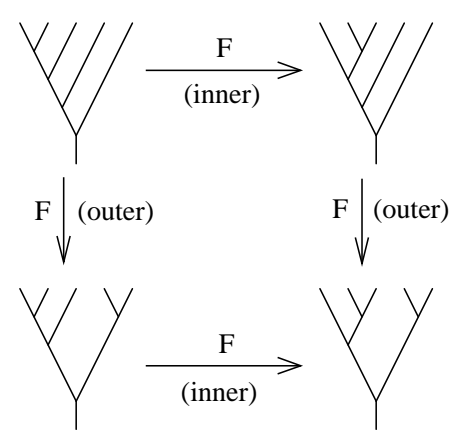

b)

Figure 17: The pentagon equation (a) and a "quadrilateral identity" (b). (The latter is satisfied automatically.)

equation and MacLane's coherence theorem, which will be formulated later. At a cruder, combinatorial level associativity may be expressed as follows: $\sum_{e} N_{a b}^{e} N_{e c}^{u}=\sum_{f} N_{a f}^{u} N_{b c}^{f}$.

From the physical perspective, splitting off (or fusing with) the vacuum sector is trivial. In any splitting diagram all lines labeled by 1 may be simply erased. To guarantee the consistency of this procedure, we require that the spaces $V_{a}^{a 1}$ and $V_{a}^{1 a}$ are not only one-dimensional, but canonically isomorphic to $\mathbb{C}$. In other words, these spaces are endowed with fixed unit vectors $\left|\alpha_{a}\right\rangle \in V_{a}^{a 1}$ and $\left|\beta_{a}\right\rangle \in V_{a}^{1 a}$. The canonical isomorphisms are given by the formulas

$$
\alpha_{a}: \mathbb{C} \rightarrow V_{a}^{a 1}: \quad z \mapsto z\left|\alpha_{a}\right\rangle, \quad \quad \beta_{a}: \mathbb{C} \rightarrow V_{a}^{1 a}: \quad z \mapsto z\left|\beta_{a}\right\rangle,
$$

where $z \in \mathbb{C}$. These isomorphisms must satisfy so-called triangle equations (see below).

Pentagon equation: The graphic representation of this axiom is shown in Fig. 17a. Its exact meaning is this: for any $x, y, z, w, u \in M$ the following diagram commutes:

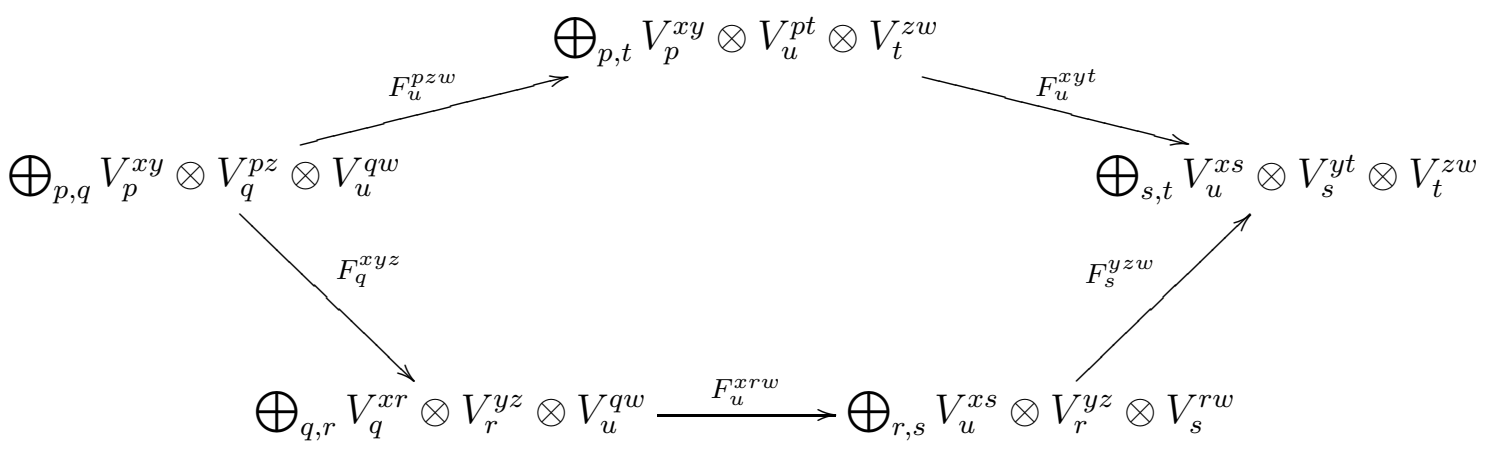

Arrow labels are abbreviated: for example, the arrow on the left (labeled by $F_{q}^{x y z}$ ) actually designates the map $\sum_{q} F_{q}^{x y z} \otimes \mathrm{id}_{V_{u}^{q w}}$, where $\mathrm{id}_{V_{u}^{q w}}$ is the unit operator on $V_{u}^{q w}$.

According to MacLane's coherence theorem [82, any sequence of $F$-moves between two given trees results in the same isomorphism between the corresponding spaces: the equality of all such isomorphisms follows from the pentagon equation. (Actually, the theorem is a bit more general and also includes $\alpha$ - and $\beta$-moves, but we ignore them for the moment.) This result 


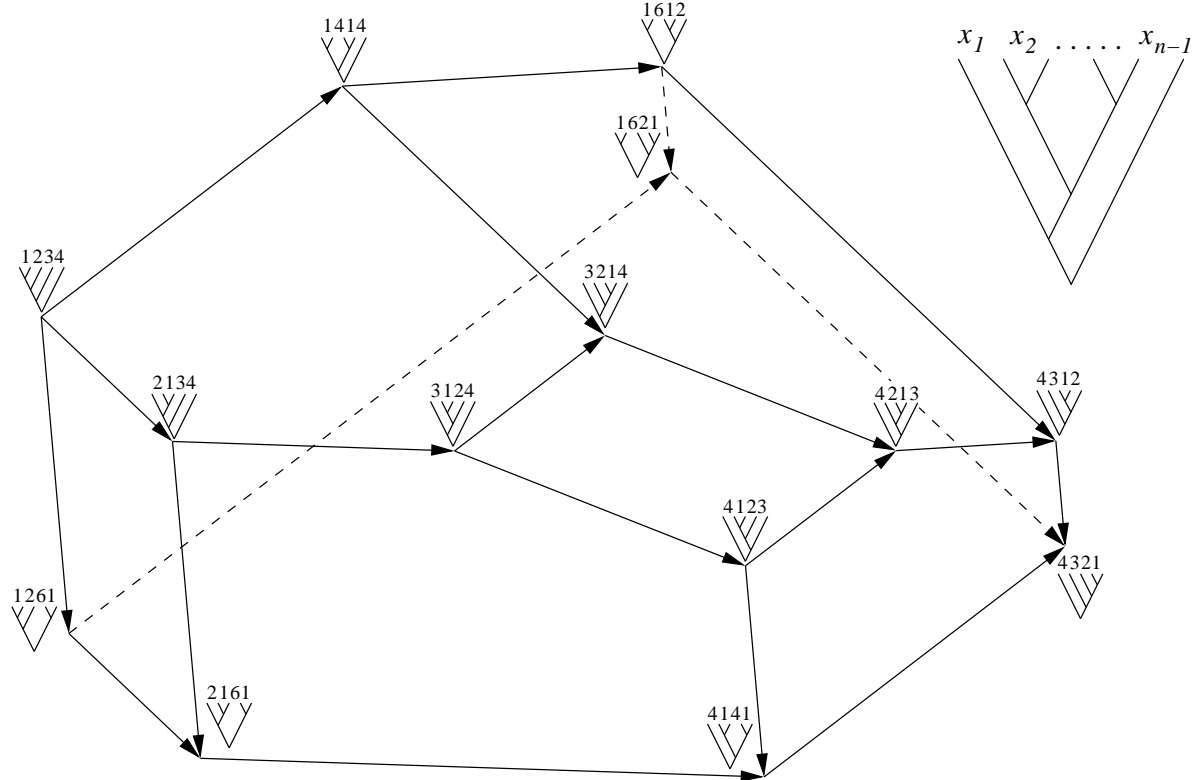

a)

$$
x_{j}=\sum_{u \in L_{j}} \sum_{v \in R_{j}} \mu_{u v},
$$

where $\mu_{u v}$ are fixed positive numbers associated with pairs of leaves (we have used $\mu_{u v}=1$ ).

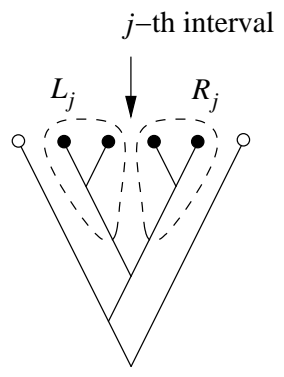

b)

Figure 18: The graph of $F$-moves between binary planar trees with $n=5$ leaves (a) and the procedure used to assign coordinates $x_{1}, \ldots, x_{n-1}$ to each tree (b).

may be regarded as a combinatorial statement. Indeed, let us consider the graph $\Gamma_{n}$ whose vertices are binary planar trees with $n$ leaves and whose edges correspond to $F$-moves. (The graph $\Gamma_{5}$ is shown in Fig. 18 a.) Then the theorem says that any cycle in the graph can be filled with pentagons and quadrilaterals. The latter correspond to the obvious fact that independent $F$-moves commute; such moves can be nested as in Fig. 17b or occur in disjoint branches. MacLane actually shows that any two directed paths between the same pair of vertices can be transformed one to the other using the pentagon and quadrilateral equations; the proof is by induction on a suitable parameter.

Let us also mention a beautiful geometric proof of the coherence theorem: the graph $\Gamma_{n}$ together with the set of 2-cells (i.e., the pentagons and quadrilaterals) can be realized by the vertices, edges, and 2-faces of some convex polytope $K_{n}$ of dimension $n-2$. This polytope appeared in the work of Stasheff [83] and now bears his name; it is also called associahedron. More exactly, this term refers to a certain combinatorial type of a polytope while its exact shape may vary. The faces of $K_{n}$ in all dimensions are associated with general (not necessarily binary) planar trees. In particular, the edges correspond to trees with one degree-3 vertex, the quadrilaterals correspond to trees with two degree-3 vertices, and the pentagons correspond to trees with one degree-4 vertex. Described in Fig. 18b is a concrete geometric realization of $K_{n}$ (cf. Ref. [84]). It is fairly easy to show that the convex hull of the points $\left(x_{1}, \ldots, x_{n-1}\right) \in \mathbb{R}^{n-1}$ obtained this way is characterized by one equation and $\frac{n(n-1)}{2}-1$ inequalities:

$$
\sum_{j=1}^{n-1} x_{j}=\eta_{1 n}, \quad \sum_{j=k}^{l-1} x_{j} \geqslant \eta_{k l} \quad(1 \leqslant k<l \leqslant n), \quad \text { where } \quad \eta_{k l} \stackrel{\text { def }}{=} \sum_{k \leqslant u<v \leqslant l} \mu_{u v} .
$$




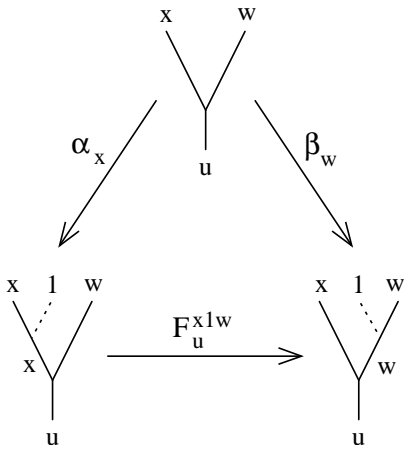

a)

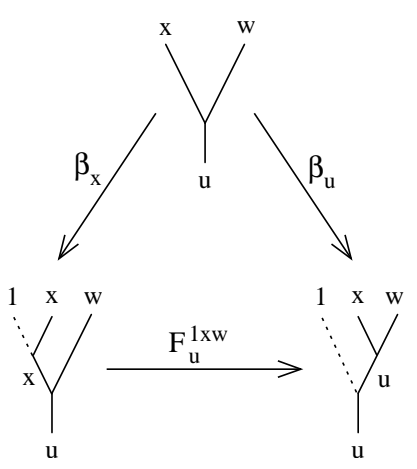

b)

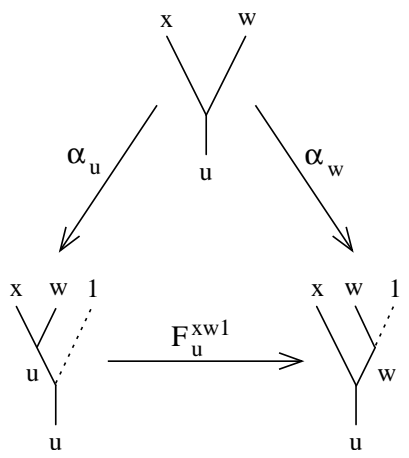

c)

Figure 19: The fundamental triangle equation (a) and its corollaries (b and c).

Triangle equations: The three equations in Fig. 19] guarantee that adding or removing trivial lines commutes with $F$-moves. Let us write the first equation in a more conventional form:

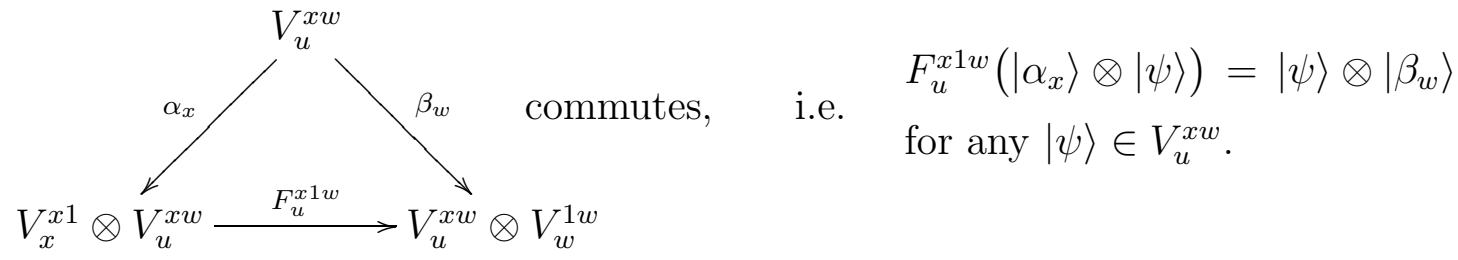

Lemma E.1. $\quad \alpha_{1}=\beta_{1}$.

Proof. Let $x=u$ and $w=1$. Then first and the third triangle equations coincide, except that the right arrow is $\beta_{1}$ in Fig. 19 and $\alpha_{1}$ in Fig. 19k. But the right arrow is the composition of the other two, therefore it is the same in both cases.

Lemma E.2 (cf. 80, Lemma XI.2.2). The first triangle equation (together with the pentagon and quadrilaterals) implies the second and the third.

The statement of the lemma refers to formal tree calculus. Moves between trees are bidirectional (therefore we may represent them by lines rather then arrows). The quadrilaterals express the commutativity of disjoint $F_{-}, \alpha^{-}$, and $\beta$-moves.

Proof. Let us prove the equation in Fig. [19].

First, we join an additional trivial branch to the trunk of each tree and show that the resulting equation is equivalent to the original one. In the diagram on the right, the old and the new equation constitute the top and the bottom of a triangular prism, respectively. The sides of the prism are commutative quadrilateral, therefore the top triangle commutes if and only if the bottom one does.

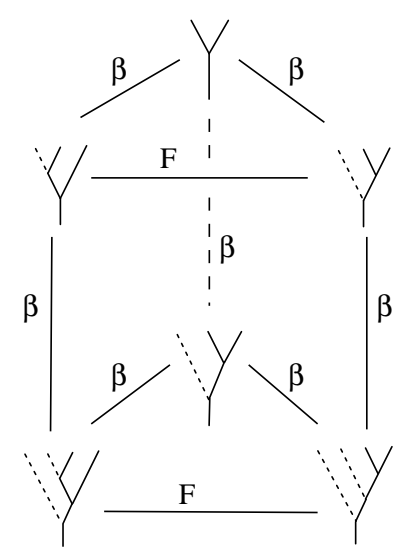


We now consider the bottom of the prism, which may be identified with triangle 4-7-5 in the new diagram. In this part of the argument it is not important that the leftmost branch of each tree is trivial, so we represent it by a solid line. The outline of the diagram corresponds to the pentagon equation. Triangles 3-1-6 and 4-2-5 are instances of the equation in Fig. 19], which is true by hypothesis. The quadrilaterals 1-2-4-3 and 6-3-4-7 also commute. Thus the required triangle 4-7-5 commutes as well.

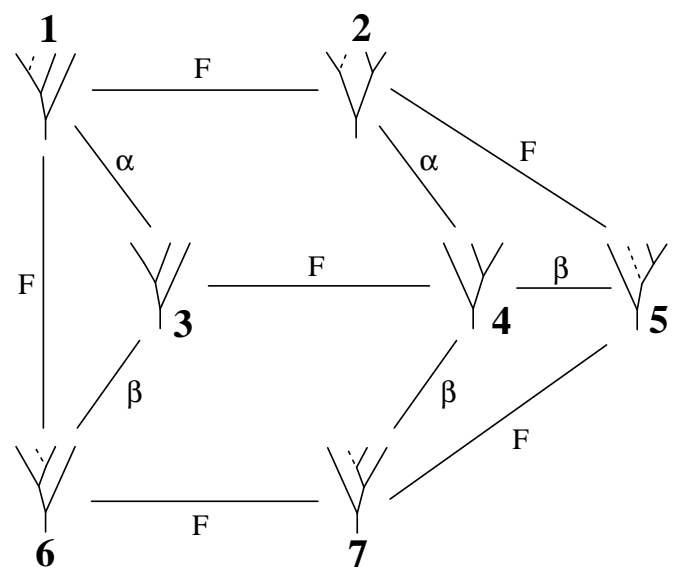

The equation in Fig. 19r is proved analogously.

E.1.3 Examples. Several concrete theories have been presented in the main text, see tables on pages [30, 41, 42, Let us now describe some general constructions leading to infinite series of examples.

1. Let the label set $M$ be a finite group and the fusion rules correspond to the group multiplication. That is, the space $V_{z}^{x, y}$ is one-dimensional (with a basis vector $\psi_{z}^{x, y}$ ) if $z=x y$, otherwise $V_{z}^{x, y}=0$. The associativity relations are trivial. Note that if the group $M$ is non-Abelian, this fusion theory does not admit braiding.

2. The associativity constraints in the above example can be deformed as follows:

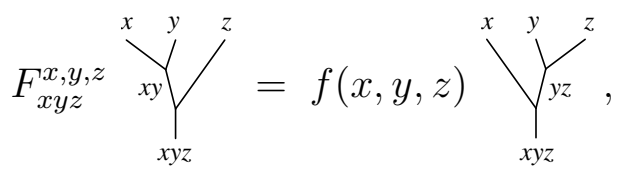

where the vertices of the trees correspond to basis vectors and $f(x, y, z)$ are some phase factors. In this case the pentagon equation reads:

$$
f(x y, z, w) f(x, y, z w)=f(x, y, z) f(x, y z, w) f(y, z, w) .
$$

This is a cocycle equation, i.e., $f$ is a 3 -cocycle on $M$ with values in $\mathrm{U}(1)$. One can show that the triangle equations do not put any additional restriction on $f$ but rather define $\alpha$ and $\beta$ :

$$
\alpha_{x}=\zeta f(x, 1,1)^{-1} \psi_{x}^{x, 1}, \quad \beta_{w}=\zeta f(1,1, w) \psi_{w}^{1, w}, \quad(\zeta \text { is an arbitrary phase }) .
$$

The basis change $\psi_{x y}^{x, y} \rightarrow u(x, y) \psi_{x y}^{x, y}$ will result in a new cocycle $g$ such that

$$
f(x, y, z)^{-1} g(x, y, z)=u(x, y) u(x, y z)^{-1} u(x y, z) u(y, z)^{-1} .
$$

The right-hand side of this equation is the coboundary of the 2-cochain $u$, i.e., $g$ and $f$ belong to the same cohomology class $\widetilde{f}$. Thus, the associativity relations are classified by $H^{3}(M, \mathrm{U}(1))$. 
For a concrete example, let $M=\mathbb{Z}_{2}=\{1, a\}$. It is known that $H^{3}\left(\mathbb{Z}_{2}, \mathrm{U}(1)\right)=\mathbb{Z}_{2}$, i.e., Eq. (183) has one trivial and one nontrivial solution. The nontrivial solution is given by $f(a, a, a)=-1$, the other seven values being equal to 1 . This particular fusion theory admits two braidings: the counterclockwise exchange of two $a$-particles is characterized by either $+i$ or $-i$. Such particles are called semions. Recall that Case 2 in the table on page 41 represents two independent species of semions.

3. Let $M$ be the set of irreducible representations of a finite group $G$ and let $V_{c}^{a b}=$ $\operatorname{Hom}_{\mathbb{C}[G]}(c, a \otimes b)$. In other words, elements of $V_{c}^{a b}$ are intertwiners between $c$ and $a \otimes b$, i.e., linear maps that commute with the group action. The associativity relations are given by $6 j$-symbols. This theory admits trivial braiding.

4. Examples 3 and 1 (with $M=G$ ) can be combined in what is called representation theory of Drinfeld's quantum double [85, 80. Models with anyons described by this theory were proposed in 86] (gauge-symmetric Lagrangian) and in 24] (lattice Hamiltonian not constrained by an external symmetry). This construction can be deformed by an arbitrary cohomology class $\tilde{f} \in H^{3}(G, \mathrm{U}(1))$, see Refs. 87, 88,

5. A very interesting set of fusion theories is based on a Temperley-Lieb category, see e.g. Chapter XII in book [68]. These theories are also known as "representations of quantum $\mathrm{SU}(2) "$.

E.1.4 Calculations with planar graphs. The rules we have described allow us to work not only with trees but with arbitrary oriented planar graphs. In particular, loops can be removed using Eq. (174). If we encounter a subgraph like shown in Eq. (174) but with different labels at the top and at the bottom (say, $c^{\prime}$ and $c^{\prime \prime}$ ), then its value is zero. Let us illustrate that by a concrete example, using the fusion theory from Table 1 on page 30 for $\varkappa=+1$ :

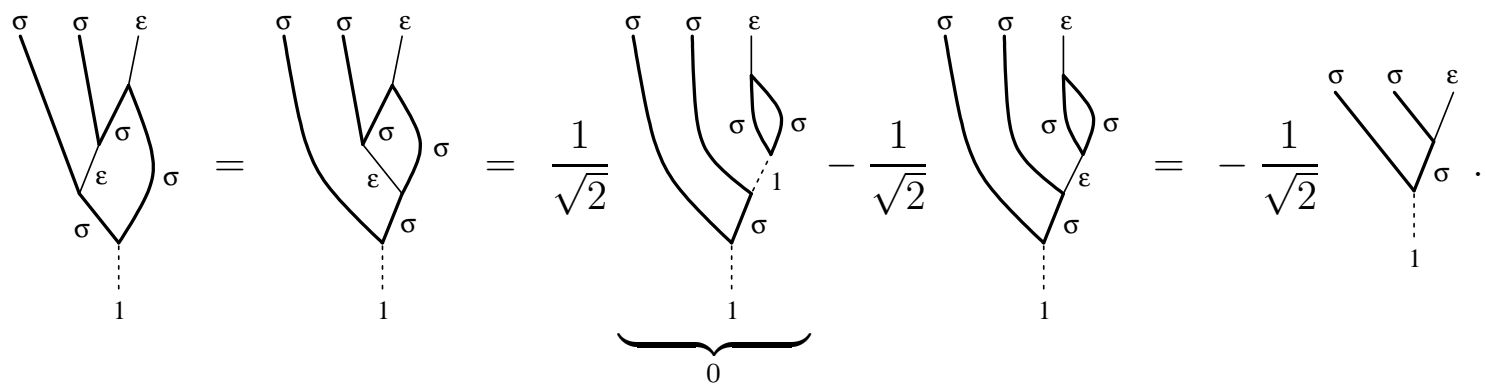

In general, we consider planar graphs that satisfy the following conditions.

1. Vertices are associated with fusion/splitting spaces $V_{l_{1}, \ldots, l_{r}}^{u_{1}, \ldots, u_{s}}$; the indices are represented by prongs. There may also be external labels (terminals) at the bottom and the top of the graph. Bottom terminals and upper indices are called sources, lower indices and top terminals are called targets.

2. Each edge connects a source to an identically labeled target. (In pictures we use a single label for the whole edge.)

3. The edges are transversal to the horizontal direction and oriented upwards. (We do not show the orientation in drawing because it is obvious.) 
To each vertex we also assign an element of the corresponding space $V_{l_{1}, \ldots, l_{r}}^{u_{1}, \ldots, u_{s}}$. Using associativity relations and Eqs. (1744), (176)), we can compute the value of the graph, $X \in V_{b_{1}, \ldots, b_{m}}^{a_{1}, \ldots, a_{k}}$, where $a_{1}, \ldots, a_{k}$ and $b_{1}, \ldots, b_{m}$ are the external labels. Note that we may freely add or remove edges labeled with 1 , thanks to the triangle equations.

A general calculation strategy is based on the idea that $X$ can be represented by a set of maps $X_{c}: V_{c}^{b_{1}, \ldots, b_{m}} \rightarrow V_{c}^{a_{1}, \ldots, a_{k}}$ for each label $c$; this representation is closely related to the decomposition of identity (176). Thus we apply Eq. (176) first, and then start shrinking and removing loops as illustrated above. For example:

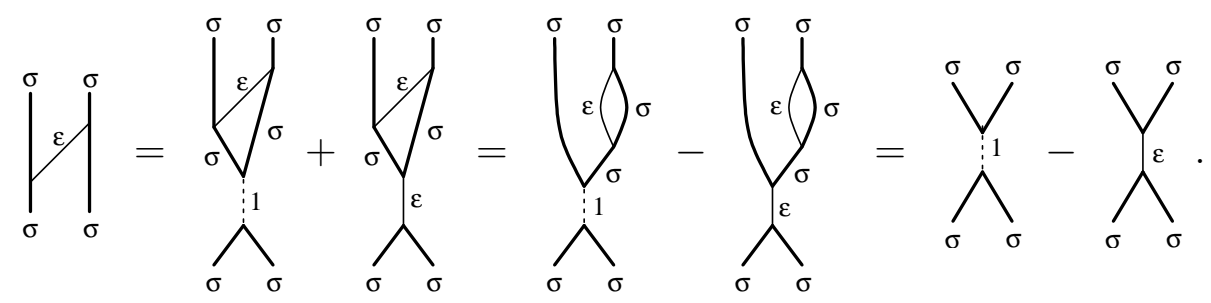

We will see that for theories with particle-antiparticle duality, condition 3 can be dispensed with. The formalism will be revised so that edges can bend, changing their direction from upward to downward and back, and vertices can rotate by $360^{\circ}$ as in Fig. 16b.

\section{E.2 Particle-antiparticle duality}

In this section we complement the fusion theory by the following condition.

Duality axiom. For each label a there is some label $\bar{a}$ and vectors $|\xi\rangle \in V_{1}^{a \bar{a}},|\eta\rangle \in V_{1}^{\bar{a} a}$ such that

$$
\left\langle\alpha_{a} \otimes \eta\left|F_{a}^{a \bar{a} a}\right| \xi \otimes \beta_{a}\right\rangle \neq 0 .
$$

Note that the matrix element in question corresponds to a physical process in which the $\bar{a}$ particle from an $a \bar{a}$-pair annihilates with a different copy of $a$ :

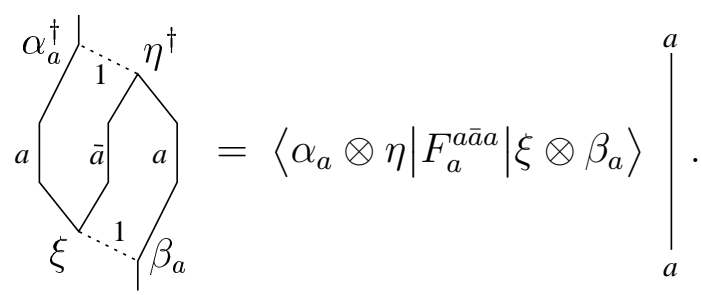

(The dotted lines and the associated labels, i.e., $1, \alpha_{a}, \beta_{a}$ may be ignored.)

Let $\left\langle\alpha_{a} \otimes \eta\left|F_{a}^{a \bar{a} a}\right| \xi \otimes \beta_{a}\right\rangle=u$, assuming that $|\xi\rangle$ and $|\eta\rangle$ are unit vectors. We will see that the spaces $V_{1}^{a \bar{a}}$ and $V_{1}^{\bar{a} a}$ are one-dimensional, therefore $|u|$ is uniquely defined. The number $d_{a}=|u|^{-1}$, called quantum dimension, plays an important role in the theory. In particular, $d_{a}=1$ for Abelian particles (i.e., such that $a \times \bar{a}=1$ ), otherwise $d_{a}>1$. If $\bar{a}=a$, then we may set $|\xi\rangle=|\eta\rangle$ so that $u$ itself has an invariant meaning. Specifically,

$$
\bigwedge_{a}^{a}=\left.\frac{\varkappa_{a}}{d_{a}}\right|_{a} ^{a},
$$


where $\varkappa_{a}$ is a uniquely defined phase, which is actually equal to \pm 1 (see below). This number is called Frobenius-Schur indicator; in the present context it was introduced by Fredenhagen, Rehren, and Schroer 89.

E.2.1 Physical motivation for the duality axiom. The existence of antiparticles follows from a locality principle mentioned at the beginning of this appendix: a quasiparticle can be moved from one place to another by applying an operator that acts on spins along a path connecting the given points. (We are still considering anyons on a line, therefore the path is unique.) The action on different spins can be performed at once or in any particular order. For example, to move a particle from point 3 to point 1 on its left, it may be natural to move it first to some middle point 2 , and then to the final destination. But it is also possible to act on the interval $[1,2]$ before $[2,3]$. The intermediate state must be a particle-antiparticle pair.

For a slightly more rigorous argument, let $X$ be an operator that moves a particle of type $a$ from 3 to 1 , acting on spins along the interval. We can represent it as follows:

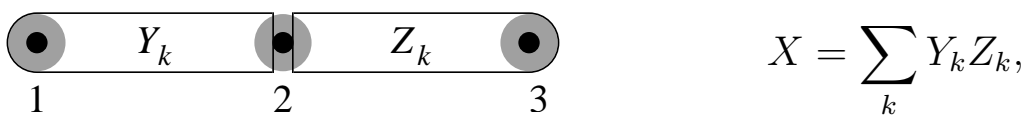

where $Y_{k}$ and $Z_{k}$ act on disjoint sets of spins in some regions around [1,2] and [2,3], respectively. Let $\Pi_{[s]}^{x}(s=1,2,3)$ be the projector onto states having particle $x$ at point $s$; such operators can be realized locally (to act in the gray circles in the above picture) if we only allow states that are not excited away from the given three points. Finally, we define new versions of $X$, $Y_{k}$, and $Z_{k}$ :

$$
\begin{gathered}
X^{\prime}=\Pi_{[1]}^{a} \Pi_{[2]}^{1} \Pi_{[3]}^{1} X \Pi_{[1]}^{1} \Pi_{[2]}^{1} \Pi_{[3]}^{a}=\sum_{k} Z_{k}^{\prime} Y_{k}^{\prime}, \\
Z_{k}^{\prime}=\Pi_{[2]}^{1} \Pi_{[3]}^{1} Z_{k} \Pi_{[3]}^{a}, \quad Y_{k}^{\prime}=\Pi_{[1]}^{a} Y_{k} \Pi_{[1]}^{1} \Pi_{[2]}^{1} .
\end{gathered}
$$

The operator $X^{\prime}$ still moves $a$ from 2 to 3 , but $Y_{k}^{\prime}$ and $Z_{k}^{\prime}$ overlap geometrically and therefore no longer commute. It is clear that each operator $Y_{k}^{\prime}$ creates an $a$-particle at point 1 and some particle at point 2, whereas $Z_{k}^{\prime}$ removes the second particle as well as the original particle at point 3. Thus each product $Z_{k}^{\prime} Y_{k}^{\prime}$ effects the process shown in Eq. (186), up to an overall factor. This factor is nonzero for at least one value of $k$.

\section{E.2.2 Some properties and normalization conventions.}

Lemma E.3. For each label a the corresponding label $\bar{a}$ is unique. Moreover, $N_{a b}^{1}=N_{b a}^{1}=\delta_{b \bar{a}}$.

Proof. Recall that $N_{a b}^{1} \stackrel{\text { def }}{=} \operatorname{dim} V_{a b}^{1}=\operatorname{dim} V_{1}^{a b}$. We first show that this number is equal to one if $b=\bar{a}$ and zero otherwise. Let $\xi, \eta$ be as in the duality axiom, and let $u$ be the corresponding matrix element. For an arbitrary element $\psi \in V_{1}^{a b}$ we have:

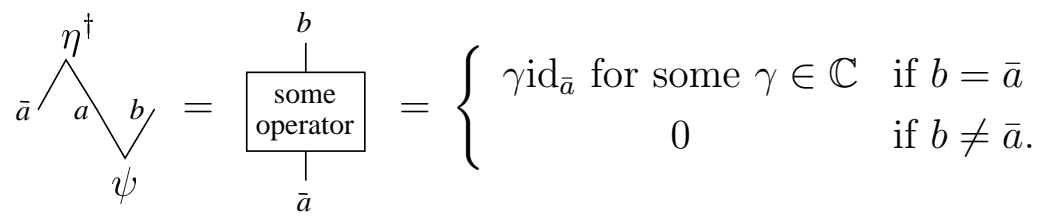


Therefore

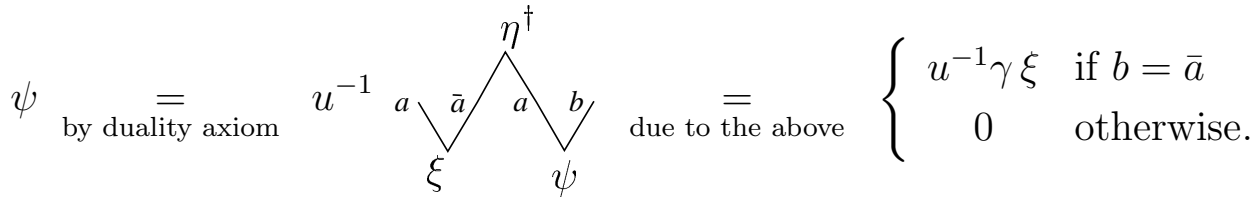

To prove that $N_{b a}^{1}=\delta_{b \bar{a}}$, we do a similar calculation with $\psi^{\prime} \in V_{1}^{b a}$, putting $\left(\psi^{\prime}\right)^{\dagger}$ on the left of $\xi$.

Corollary E.4. $\overline{1}=1$ and $\overline{\bar{a}}=a$.

Let us now set new normalization conventions. In the definition of $d_{a}$ we assumed that $|\xi\rangle$ and $|\eta\rangle$ are unit vectors, i.e., $\xi^{\dagger} \xi=\eta^{\dagger} \eta=\mathrm{id}_{1}$. However, it is more convenient to multiply $\xi$ and $\eta$ by $\sqrt{d_{a}}$ (and some suitable phase factors) so that the zigzag on the left-hand side of equation (186) could be simply removed. The so normalized operators for the creation of particle-antiparticle pairs will be represented as smooth "cups" with a triangle mark at the bottom. The adjoint operators are "caps" obtained by flipping the pictures about a horizontal line: ${ }^{21}$

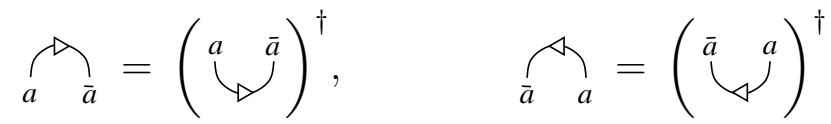

In all four cases, the triangle points from $a$ to $\bar{a}$ as we follow the line. The four operators are defined up to a single phase and satisfy the following relations:

$$
\begin{aligned}
& a \bigodot^{a}=d_{a}=\bar{a} \bigodot a
\end{aligned}
$$

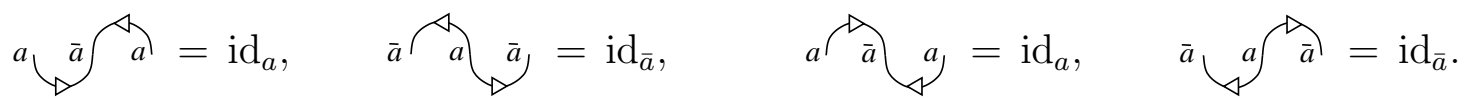

Eq. (191) and the first equality in (192) are true by definition. The second equality can be proved analogously to Lemma E.3, the last two equalities are obtained by passing to adjoint operators. Roughly, the normalization conditions may be described as follows: oppositely oriented triangles cancel each other.

So far the label $a$ was fixed. Repeating the same procedure for $\bar{a}$, we get a new set of basis vectors in $V_{1}^{a \bar{a}}, V_{1}^{\bar{a} a}, V_{a \bar{a}}^{1}, V_{\bar{a} a}^{1}$, which must be related to the old ones:

$$
\begin{aligned}
& \mho^{\bar{a}}=\varkappa_{a} \downarrow^{a}, \quad \mho^{a}=\varkappa_{\bar{a}} \swarrow^{\bar{a}}, \\
& \bigwedge_{a}=x_{a}^{*} \bigwedge_{a}, \quad \bigwedge_{\bar{a}}=x_{\bar{a}}^{*} \bigwedge_{\bar{a}}{ }_{a} .
\end{aligned}
$$

Combining these relations with Eqs. (191), (192), we get:

$$
\left|\varkappa_{a}\right|^{2}=d_{\bar{a}} / d_{a}=\left|\varkappa_{\bar{a}}\right|^{-2}, \quad \varkappa_{a}\left(\varkappa_{\bar{a}}^{*}\right)^{-1}=1 .
$$

\footnotetext{
${ }^{21}$ This notation is not standard, but it is convenient for calculations. In the notation used in 68, 79, 80, cups and caps are not decorated but each particle has a fictitious degree of freedom: it is considered as either $a$ going up or $\bar{a}$ going down. This prevents noninvariant phases from appearing in formulas.
} 
Thus,

$$
d_{\bar{a}}=d_{a}, \quad \varkappa_{\bar{a}}=\varkappa_{a}^{*}, \quad\left|\varkappa_{a}\right|=1 .
$$

In general, the number $\varkappa_{a}$ depends on the arbitrary phases in the definition of cups and caps. However, if $a=\bar{a}$, then $\varkappa_{a}$ is defined uniquely and coincides with the Frobenius-Schur indicator. ${ }^{22}$ In this case, $\varkappa_{a}= \pm 1$.

Now we are in a position to relax the requirement that edges are transversal to the horizontal direction.

Definition-Proposition E.5. A line is an alternating sequence of $2 n$ cups and caps without triangle marks. It may be open or closed. Such an object has a canonical normalization given by $n$ triangles pointing forward and $n$ triangles pointing backward (relative to a chosen path direction). All such decorations are equivalent.

Let us also renormalize the inner product on splitting spaces:

$$
\left.\langle\langle\eta \mid \xi\rangle\rangle \stackrel{\text { def }}{=} \sqrt{\frac{d_{c}}{d_{a} d_{b}}}\langle\eta \mid \xi\rangle=\frac{1}{\sqrt{d_{a} d_{b} d_{c}}} \stackrel{\eta^{\dagger}}{\xi} \bigcap_{c}^{c}\right\}_{c}{ }_{c}, \quad \text { where } \quad \xi, \eta \in V_{c}^{a b} .
$$

(Triangles may be added in one of the two consistent ways; one may also flip the $c$-loop to the left.) This renormalization does not violate the unitarity of associativity relations. The inner product on fusion spaces is defined through the antilinear isomorphism $V_{c}^{a b} \rightarrow V_{a b}^{c}: \psi \mapsto \psi^{\dagger}$. When computing either type of inner product, we stack two trees with branches touching each other and connect the roots by a loop. Stacking the trees root to root might produce a different result, but it turns out to be the same since we can actually rotate vertices by $360^{\circ}$, see below.

Finally, we rewrite Eq. (176) in a form that is consistent with the new rules:

$$
\left.\left.\right|_{a} ^{a}\right|_{b} ^{b}=\sum_{c} \sum_{j} \sqrt{\frac{d_{c}}{d_{a} d_{b}}} \underbrace{a}_{c} \underbrace{b}_{b, j}\left(\psi_{c, j}^{a b} \psi_{b}^{\dagger a}\right)^{\dagger}, \quad \text { where }\left\langle\left\langle\psi_{c, j}^{a b} \mid \psi_{c, k}^{a b}\right\rangle\right\rangle=\delta_{j k}
$$

(the elements $\psi_{c, j}^{a b} \in V_{c}^{a b}$ form a complete basis).

E.2.3 Raising and lowering of indices. Let us define the following linear maps:

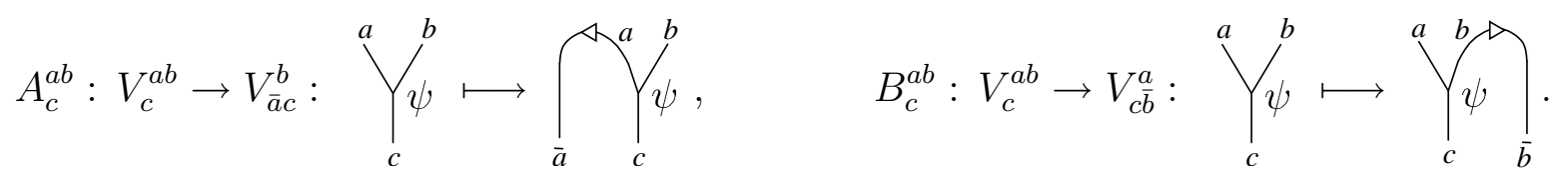

They are obviously invertible, hence $N_{a b}^{c}=N_{\bar{a} c}^{b}=N_{b \bar{c}}^{\bar{a}}=N_{\bar{b} \bar{a}}^{\bar{c}}=N_{\bar{c} a}^{\bar{b}}=N_{c \bar{b}}^{a}$. Unfortunately, this does not save us from the need to to distinguish between lower and upper indices because the

\footnotetext{
${ }^{22}$ In category theory, $\varkappa_{a}$ is not a scalar but rather a morphism from $a$ to $\overline{\bar{a}}$, which are regarded as different (albeit isomorphic) objects. Thus the arbitrariness goes away, but the Frobenius-Schur indicator has to be defined in a more complicated fashion.
} 


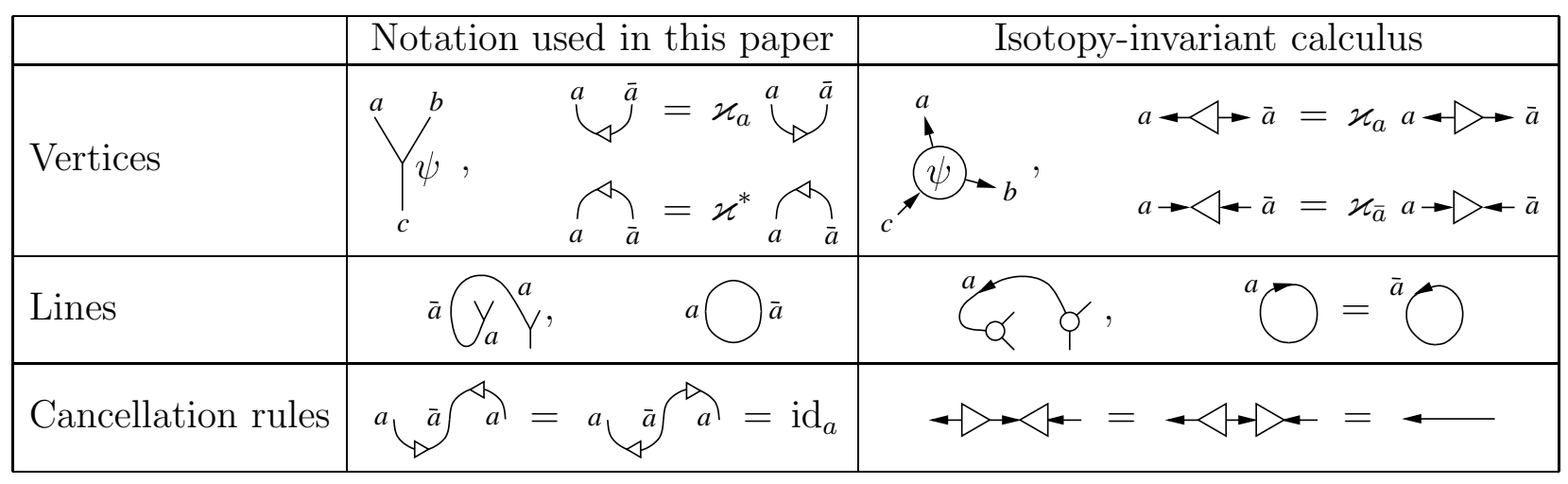

Table 5: Notation summary.

simultaneous raising and lowering of indices on the two ends of a line results in the factor $\varkappa_{a}$. For example:

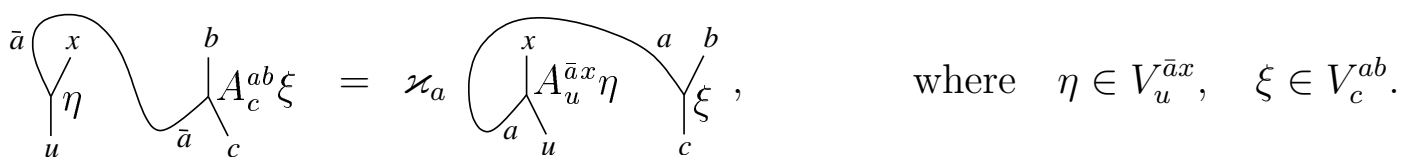

However, as the following theorem shows, we do not have to tie the types of indices to the orientation of the corresponding vertex. This way, one obtains an isotopy-invariant calculus for planar graphs, see Table 5. We will continue using the previous notation, though.

Theorem E.6. The maps $A_{c}^{a b}, B_{c}^{a b}$ are unitary with respect to the inner product $\langle\langle\cdot \mid \cdot\rangle\rangle$, and the following diagram (in which the arrows may be traversed in both directions) commutes:

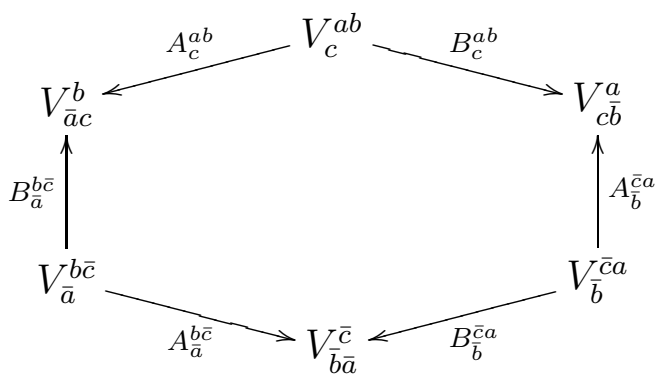

Note that the commutativity of this diagram is equivalent the "pivotal identity" in Fig 16b. The proof of the theorem is preceded by two lemmas.

Lemma E.7. The composition of any two adjacent arrows in Eq. (199) is unitary.

Proof. Due to symmetry, it suffices to consider just one particular case, e.g., the arrows connected at the upper left corner. Let $X=\left(B_{\bar{a}}^{b \bar{c}}\right)^{-1} A_{c}^{a b}: V_{c}^{a b} \rightarrow V_{\bar{a}}^{b \bar{c}}$. For arbitrary elements $\xi \in V_{c}^{a b}, \eta \in V_{\bar{a}}^{b \bar{c}}$ we have:

$$
X \xi=\bigvee_{\bar{a}}^{a} \int_{\xi}^{b}, \quad X^{-1} \eta=\underbrace{a}_{\bar{a}} \bigvee_{c}^{b}
$$




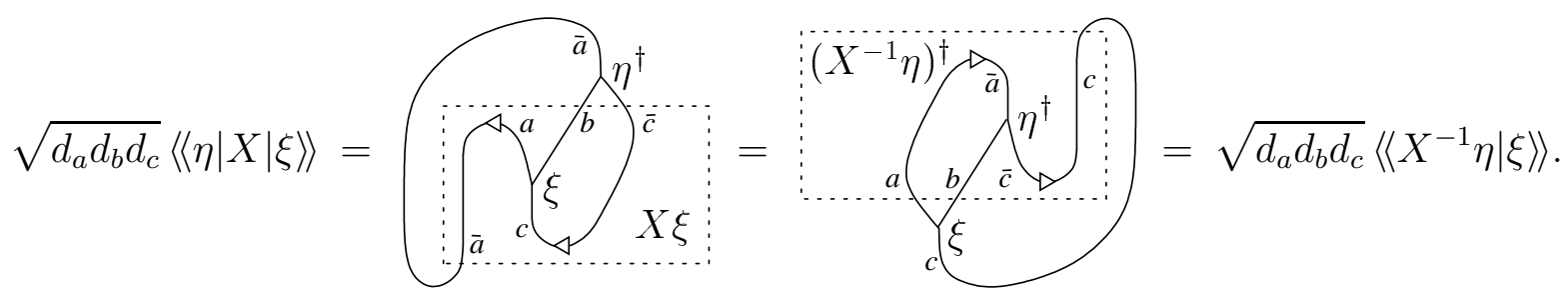

Therefore $X^{-1}=X^{\dagger}$.

Now consider the two paths from top to bottom of the hexagon (199):

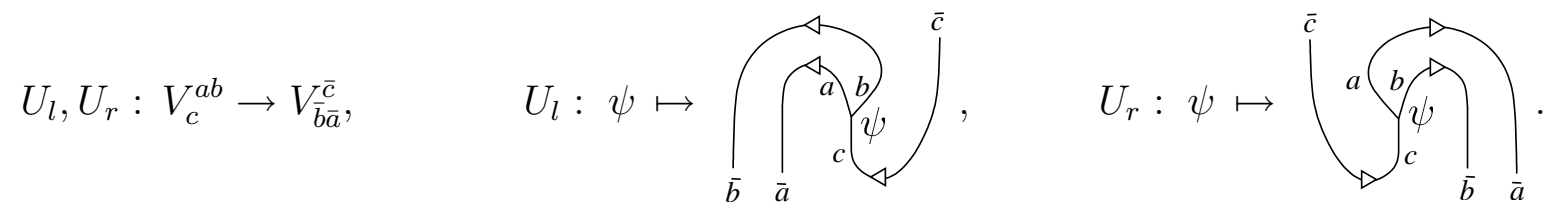

From the physical point of view, these operators (which are actually equal) correspond to the CPT symmetry.

Lemma E.8. $U_{l}^{\dagger}=U_{r}^{-1}$.

Proof. Let $\xi \in V_{c}^{a b}$ and $\eta \in V_{\bar{b} \bar{a}}^{\bar{c}}$. Then

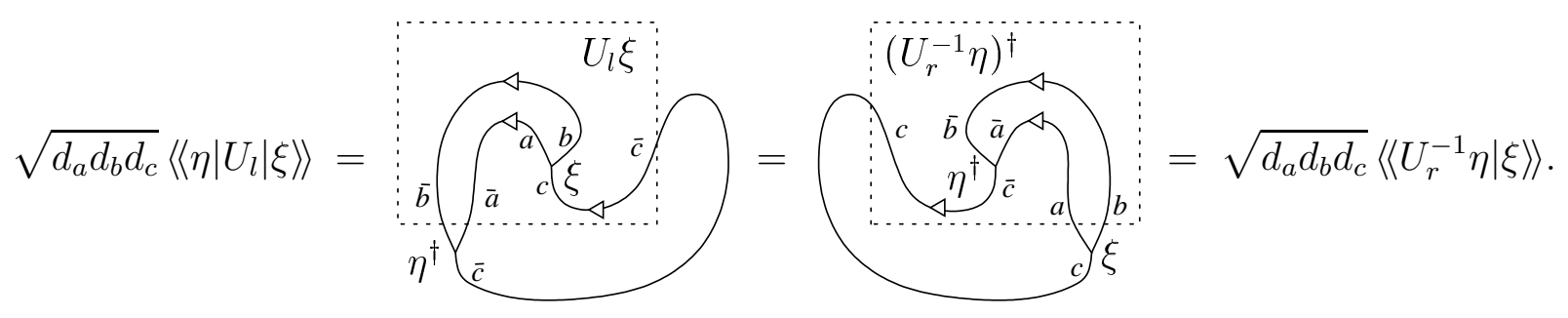

Proof of Theorem E.6. Let us consider the path around the diagram (199) in the counterclockwise direction, $W=U_{r}^{-1} U_{l}$. This operator is a composition of six arrows, therefore it is unitary (due to Lemma E.7). On the other hand, Lemma E.8 implies that $W=U_{l}^{\dagger} U_{l}$ is Hermitian and positive semidefinite. It follows that $W$ is the identity operator, i.e., the diagram commutes.

Thus, $U_{l}=U_{r}$; let us denote this operator simply by $U$. It is unitary by Lemma E.8. Any arrow in the diagram is a composition of $U$ ( or $U^{-1}$ ) and some number of arrow pairs. Therefore all the arrows are unitary.

E.2.4 Quantum dimension and fusion rules. This is a key identity:

$$
d_{a} d_{b}=\sum_{c} N_{a b}^{c} d_{c}
$$

To prove it, we use Eq. (196), the pivotal property, and Eq. (195):

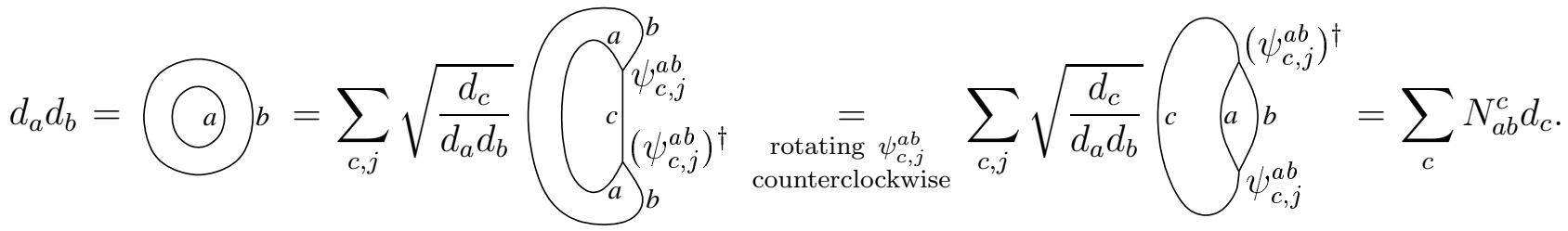


Eq. (201) implies that the matrix $\widehat{N}(a)=\left(N_{a b}^{c}: b, c \in M\right)$ has eigenvector $v=\left(d_{c}: c \in M\right)$, and the corresponding eigenvalue is $d_{a}$. Note that all the entries of $v$ are positive. According to the Perron-Frobenius theorem (about matrices with nonnegative entries), all eigenvalues of $\widehat{N}(a)$ satisfy the inequality

$$
\left|\lambda_{j}(a)\right| \leqslant d_{a}
$$

Thus, the quantum dimension $d_{a}$ is the largest eigenvalue of the matrix $\widehat{N}(a)$.

The proof of Eq. (201) also motivates a definition of quantum trace. It is a number assigned to any element $X \in V_{a_{1} \ldots a_{n}}^{a_{1} \ldots a_{n}}$. Such an element acts as an operator in the space $V_{c}^{a_{1} \ldots a_{n}}$ for each $c$; we denote this action by $X_{c}$. The quantum trace is defined as follows:

$$
\widetilde{\operatorname{Tr}} X \stackrel{\text { def }}{=} \sum_{c} d_{c} \operatorname{Tr} X_{c}={ }^{a_{1} a_{2} \ldots a_{n}} .
$$

\section{E.3 Braiding and topological spin}

Braiding is an additional piece of algebraic structure characterizing anyons. It is defined by a set of elements $R_{a b} \in V_{a b}^{b a}$ that represent transposition of two particles:

$$
R_{a b}=\sum_{a}^{b} y_{b}^{a}, \quad\left(R_{a b}\right)^{-1}=\left(R_{a b}\right)^{\dagger}=\overbrace{b}^{a} .
$$

They must satisfy the Yang-Baxter equation, i.e., a line can be moved over or under a crossing between two other lines:

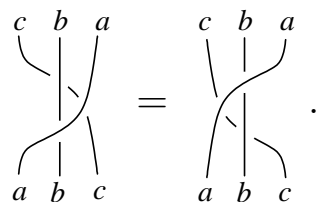

More generally, a line can slide over or under an arbitrary vertex. It is sufficient to postulate this property for three-prong vertices representing splitting and fusion and for lines tilted left and right. Furthermore, splitting and fusion are related by the decomposition of the identity (196)), whereas changing the slope of the intersecting line is equivalent to replacing $R$ with $R^{-1}$. Thus the number of independent conditions is reduced to two:
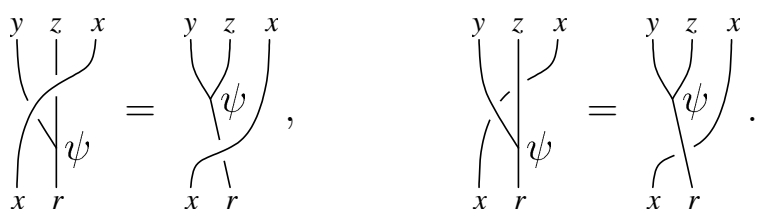

The description of braiding in terms of basic data amounts to specifying the action of $R_{a b}$ on splitting spaces:

$$
\bigodot_{c}^{b}=R_{c}^{a b} \psi, \quad \quad R_{c}^{a b}: V_{c}^{a b} \rightarrow V_{c}^{b a} .
$$




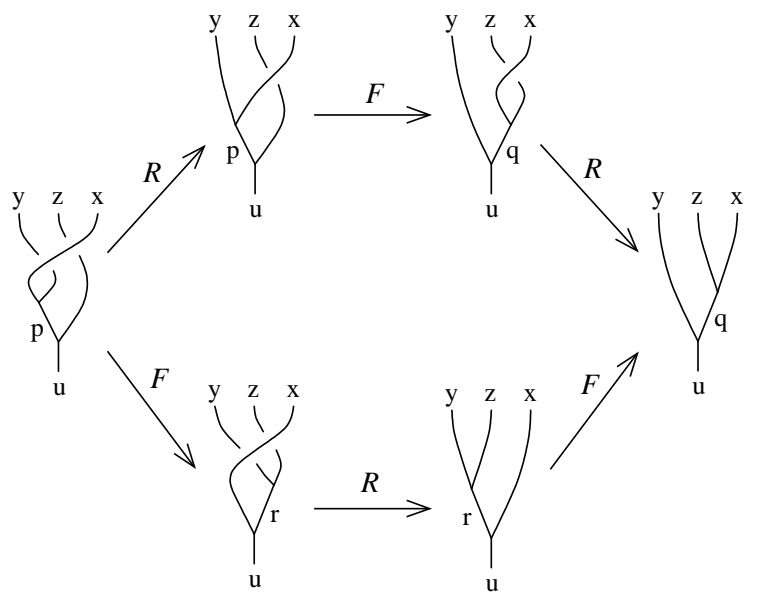

a)

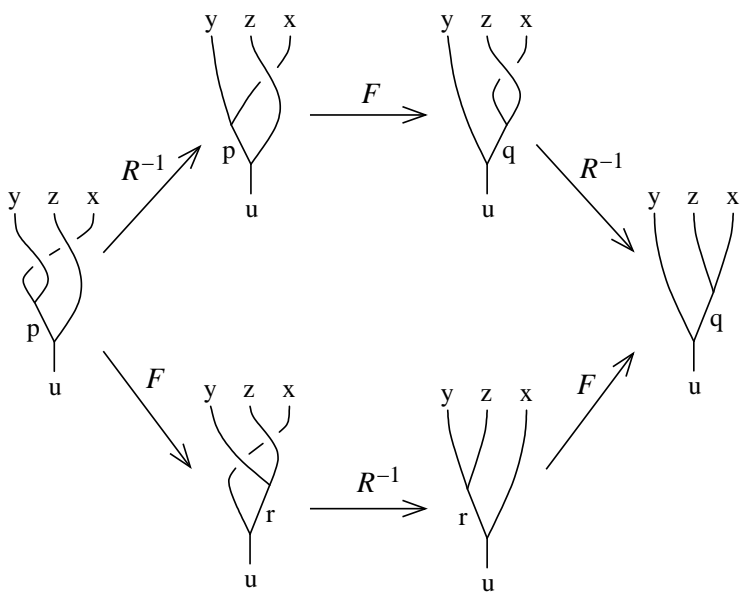

b)

Figure 20: The hexagon equations.

Note that $R_{c}^{a b}$ is a unitary map, therefore $N_{a b}^{c}=N_{b a}^{c}$.

To express Eq. (206) in terms of $R_{c}^{a b}$, we join the two lines at the bottom of each graph and perform equivalence transformations. These include $F$-moves as well as $R$-moves - absorbing a line crossing by a vertex. Thus we obtain the diagrams in Fig. 20, the bottommost arrow in each of them combines an $R$-move with the first or the second equation in question. We may now forget about the topological meaning of braiding and only keep track of the linear maps involved:

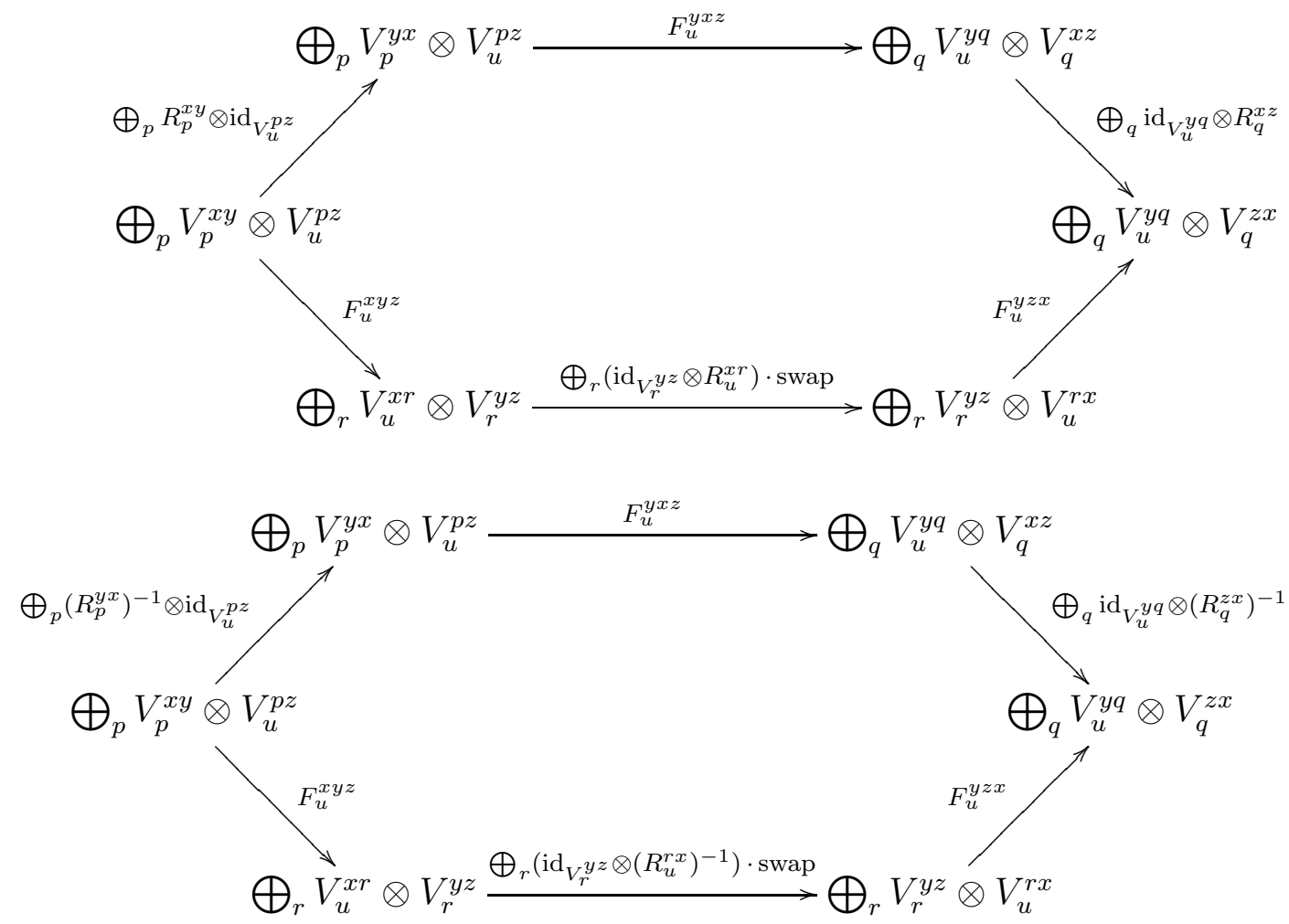

These commutative diagrams are known as hexagon equations. They actually look nicer in the tensor category formalism, see Eqs. (274) and (275). 
Note that braiding with label 1 is trivial:

$$
\zeta_{a}^{a} \alpha_{a}^{a}=\varlimsup_{a}^{1} \alpha_{a}^{a}=\int_{a}^{1} \int_{\beta_{a}}^{a}, \quad \text { i.e., } \quad R_{a}^{a 1} \alpha_{a}=\left(R_{a}^{1 a}\right)^{-1} \alpha_{a}=\beta_{a} .
$$

(The proof is analogous to that of LemmaE.2) Due to this property, we need not worry about lines labeled by 1, e.g., ones that are implicitly attached to each cup and cap.

To each label $a$ we associate a complex number $\theta_{a}$, called topological spin:

$$
\theta_{a} \stackrel{\text { def }}{=} d_{a}^{-1} \bigcirc a \bigcup_{a}=d_{a}^{-1} \widetilde{\operatorname{Tr}} R_{a a}=d_{a}^{-1} \sum_{c} d_{c} \operatorname{Tr} R_{c}^{a a} .
$$

It may also be characterized by any of the following relations:
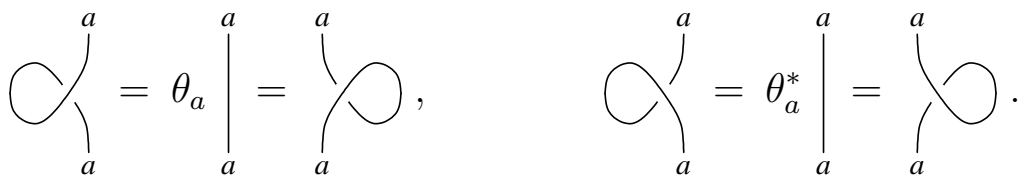

Note the following properties of the topological spin:

$$
\left|\theta_{a}\right|=1, \quad \theta_{\bar{a}}=\theta_{a} .
$$

Indeed,

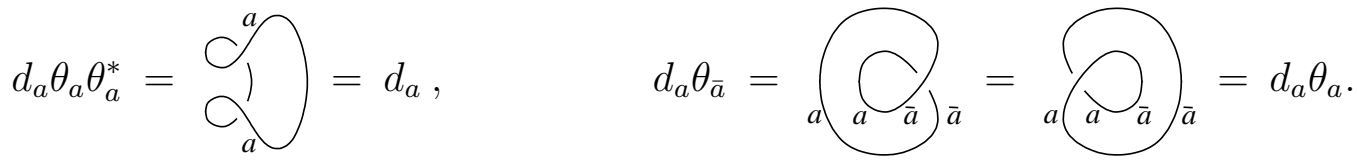

This is yet another expression for the topological spin:

$$
\bigcup_{\infty}^{a}=\theta_{a}^{*} \int^{a}
$$

(To prove it, we need to put a left-oriented cap on both sides of the equation and rotate the resultant figure-eight on the left-hand side by $90^{\circ}$.) In particular, if $\bar{a}=a$, then we have the following representation for the invariant scalar $R_{1}^{a a}$ :

$$
R_{1}^{a a}=\theta_{a}^{*} \varkappa_{a} .
$$

Remark E.9. Eq. (215) provides a simple physical interpretation for the Frobenius-Schur indicator $\varkappa_{a}$. Suppose that the system is rotationally invariant, so that not only the topological spin but also the usual spin $s_{a}$ has physical meaning. Of course, $s_{a}$ may take different values subject to the constraint $e^{2 \pi i s_{a}}=\theta_{a}$. Let us consider a pair of identical particles with trivial total topological charge. What values does the total angular momentum of this composite object take? This question may be answered as follows. Assuming that both particles are in the same spin state $s_{a}$ and barring additional "isospin" degrees of freedom, the $180^{\circ}$ rotation is characterized by the phase factor $e^{i \pi s_{a}} e^{i \pi s_{a}} R_{1}^{a a}=\varkappa_{a}$. Thus, the total angular momentum is even if $\varkappa_{a}=1$ and odd if $\varkappa_{a}=-1$. 
It is interesting that the effect of moving one particle around another is fully characterized by the topological spin:

$$
R_{c}^{b a} R_{c}^{a b}=\frac{\theta_{c}}{\theta_{a} \theta_{b}} \operatorname{id}_{V_{c}^{a b}}
$$

Proof. Consider the following element of the space $V_{a b}^{a b}$ :

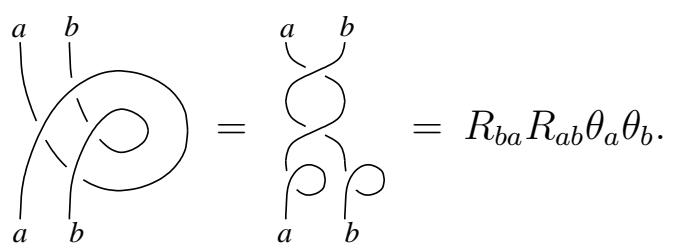

Acting by it as an operator on the space $V_{c}^{a b}$, we obtain the required identity.

Theorem E.10 (Vafa [90]). The topological spins are roots of unity. More specifically, $\theta_{a}^{n}=1$, where the integer $n \neq 0$ depends only on the fusion multiplicities.

Proof. Let us use this fact from linear algebra: for any integer matrix $X$ there is an integer matrix $Y$ (the adjugate) such that $Y X=(\operatorname{det} X) I$. Therefore, if $\prod_{b} \theta_{b}^{X_{a b}}=1$ for all $a$, then $\theta_{b}^{\operatorname{det} X}=1$ for all $b$.

To find multiplicative relations between topological spins, we consider determinants of $R$ and $F$-moves. Such determinants generally depend on the choice of basis in the source and the target space. So, let us fix a basis in each space $V_{c}^{a b}$ and replace the linear maps in the hexagon equations (208) and (209) by their determinants. Dividing the first equation by the second, we get:

$$
\operatorname{det}\left(\bigoplus_{p}\left(R_{p}^{x y} R_{p}^{y x}\right) \otimes \operatorname{id}_{V_{u}^{p z}}\right) \operatorname{det}\left(\bigoplus_{q} \operatorname{id}_{V_{u}^{y q}} \otimes\left(R_{q}^{x z} R_{q}^{z x}\right)\right)=\operatorname{det}\left(\bigoplus_{r} \operatorname{id}_{V_{r}^{y z}} \otimes\left(R_{u}^{x r} R_{u}^{r x}\right)\right) .
$$

Note that the determinants here are actually invariant and can be expressed in terms of topological spins using Eq. (216):

$$
\begin{gathered}
\prod_{p}\left(\frac{\theta_{p}}{\theta_{x} \theta_{y}}\right)^{N_{x y}^{p} N_{p z}^{u}} \prod_{q}\left(\frac{\theta_{q}}{\theta_{x} \theta_{z}}\right)^{N_{y q}^{u} N_{x z}^{q}}=\prod_{r}\left(\frac{\theta_{u}}{\theta_{x} \theta_{r}}\right)^{N_{y z}^{r} N_{x r}^{u}}, \\
\prod_{p} \theta_{p}^{N_{x y}^{p} N_{z v}^{\bar{p}}+N_{x z}^{p} N_{y v}^{\bar{p}}+N_{y z}^{p} N_{x v}^{\bar{p}}}=\left(\theta_{x} \theta_{y} \theta_{z} \theta_{v}\right)^{N_{x y z v}}, \quad N_{x y z v} \stackrel{\text { def }}{=} \sum_{q} N_{x y}^{q} N_{z v}^{\bar{q}},
\end{gathered}
$$

where we have substituted $v$ for $\bar{u}$.

For our purposes, it is sufficient to consider the case where $x=y=a$ and $z=v=\bar{a}$. Thus we obtain a system of equations described by a square matrix:

$$
\prod_{b} \theta_{b}^{X_{a b}}=1, \quad \text { where } \quad X_{a b}=4 N_{a a \bar{a} \bar{a}} \delta_{a b}-2 N_{a \bar{a}}^{b} N_{a \bar{a}}^{\bar{b}}-N_{a a}^{b} N_{\bar{a} \bar{a}}^{\bar{b}} .
$$


It remains to show that $X$ is nondegenerate. For this purpose, we may divide the $a$-th row of $X$ by $N_{a a \bar{a} \bar{a}}$ so that the matrix becomes $4 I-Y$; the eigenvalues of $Y$ are bounded by a suitable multiplicative matrix norm:

$$
Y_{a b}=\left(N_{a a \bar{a} \bar{a}}\right)^{-1}\left(2 N_{a \bar{a}}^{b} N_{a \bar{a}}^{\bar{b}}+N_{a a}^{b} N_{\bar{a} \bar{a}}^{\bar{b}}\right), \quad \quad \mid \text { eigenvalue }_{j}(Y)\left|\leqslant \max _{a} \sum_{b}\right| Y_{a b} \mid=3 .
$$

Thus the matrix $4 I-Y$ cannot have a zero eigenvalue.

\section{E.4 Verlinde algebra and topological $S$-matrix}

In this section we investigate some properties of fusion, based on the axioms stated above. First, let as consider a fusion theory with particle-antiparticle duality, but without braiding. The Verlinde algebra is an associative $*$-algebra spanned by elements $\mathbf{e}_{a}: a \in M$ which satisfy the following relations:

$$
\mathbf{e}_{a} \mathbf{e}_{b}=\sum_{c} N_{a b}^{c} \mathbf{e}_{c}, \quad \quad \mathbf{e}_{a}^{\dagger}=\mathbf{e}_{\bar{a}}
$$

The basis element $\mathbf{e}_{a} \in \operatorname{Ver}$ may be represented by the matrix $\widehat{N}(a)=\left(N_{a b}^{c}: b, c \in M\right)$; this representation is faithful. Since $\widehat{N}(\bar{a})=\widehat{N}(a)^{\dagger}$, the algebra operation $\dagger$ corresponds to taking the adjoint matrix. Therefore $\operatorname{Ver}$ is actually a finite-dimensional $C^{*}$-algebra. Equation (201) says that the linear map $\operatorname{Ver} \rightarrow \mathbb{C}: \mathbf{e}_{a} \mapsto d_{a}$ is a homomorphism.

If braiding is defined, then $N_{a b}^{c}=N_{b a}^{c}$, therefore the Verlinde algebra is commutative. A finite-dimensional commutative $C^{*}$-algebra splits into several copies of $\mathbb{C}$. Therefore we have $|M|$ distinct homomorphisms from $\operatorname{Ver}$ to $\mathbb{C}$, which we refer to as fusion characters:

$$
\lambda_{j}: \operatorname{Ver} \rightarrow \mathbb{C}, \quad \lambda_{j}(a) \lambda_{j}(b)=\sum_{c} N_{a b}^{c} \lambda_{j}(c)
$$

The vector $v_{j}=\left(\lambda_{j}(c): c \in M\right)$ is a common eigenvector of the matrices $\widehat{N}(a)$, the eigenvalues being equal to $\lambda_{j}(a)$. Note that the matrices $\widehat{N}(a)$ are normal, therefore eigenvectors corresponding to distinct eigenvalues must be orthogonal. Thus the following orthogonality condition holds:

$$
\sum_{a} \lambda_{j}(a) \lambda_{k}(a)^{*}=0 \quad \text { if } j \neq k .
$$

We will now construct a map from the set of labels to the set of fusion characters. Specifically, we are to show that some of the characters are given (up to a constant factor) by the columns of the topological $S$-matrix $S=\left(s_{a b}: a, b \in M\right)$, where

$$
s_{a b} \stackrel{\text { def }}{=} \frac{1}{\mathcal{D}} \bar{a}\left(\bar{b} a b=\frac{\widetilde{\operatorname{Tr}}\left(R_{\bar{b} a} R_{a \bar{b}}\right)}{\mathcal{D}}=\frac{1}{\mathcal{D}} \sum_{c} d_{c} \operatorname{Tr}\left(R_{c}^{\bar{b} a} R_{c}^{a \bar{b}}\right)=\frac{1}{\mathcal{D}} \sum_{c} N_{a \bar{b}}^{c} \frac{\theta_{c}}{\theta_{a} \theta_{b}} d_{c} .\right.
$$

The normalization factor $1 / \mathcal{D}$ with $\mathcal{D}=\sqrt{\sum_{a} d_{a}^{2}}$ is chosen so that $S$ is unitary under certain conditions.

Let us study properties of the numbers $s_{a b}$. First, we observe these symmetries:

$$
s_{a b}=s_{b a}=s_{\bar{a} \bar{b}}=s_{\bar{b} \bar{a}}=s_{\bar{a} b}^{*}=s_{b \bar{a}}^{*}=s_{a \bar{b}}^{*}=s_{\bar{b} a}^{*} .
$$


Indeed, up to normalization we have:

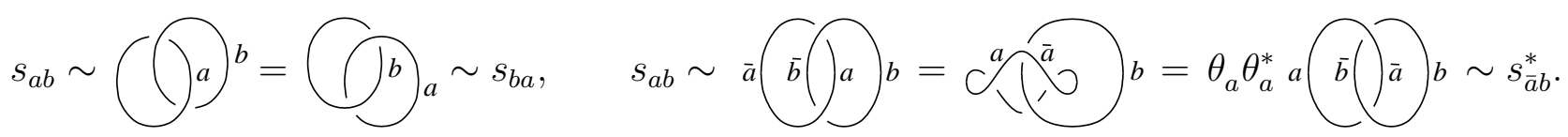

Factors of the form $s_{a x} / s_{1 x}$ often arise via this equation:

$$
\bigcup_{\bar{x}}^{\bar{x}} a=\left.\frac{s_{a x}}{s_{1 x}}\right|_{\bar{x}} ^{\bar{x}}, \quad s_{1 x}=\frac{d_{x}}{\mathcal{D}}
$$

For example, let us repeat the calculation done in proof of Eq. (201), but now with an additional line passing through the loops:

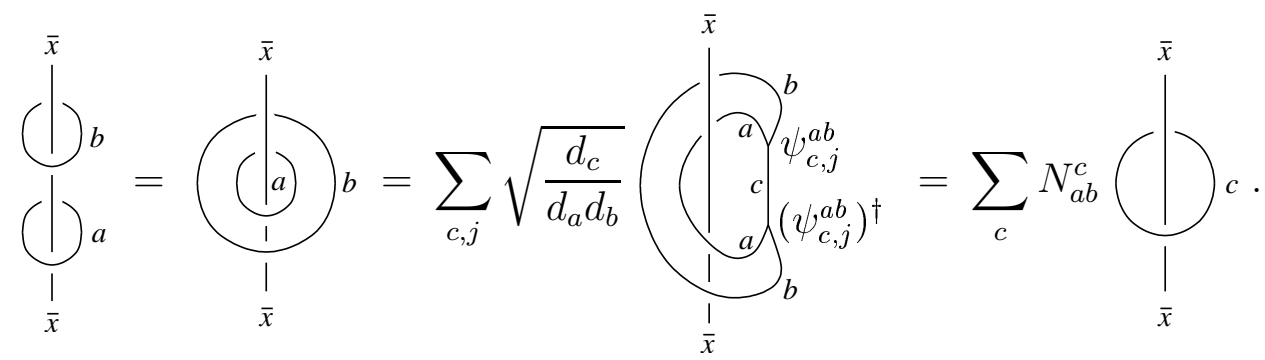

The first and the last expression are equal to $\frac{s_{a x} s_{b x}}{s_{1 x}^{2}} \mathrm{id}_{\bar{x}}$ and $\sum_{c} N_{a b}^{c} \frac{s_{c x}}{s_{1 x}} \mathrm{id}_{\bar{x}}$, respectively. Thus,

$$
\frac{s_{a x} s_{b x}}{s_{1 x}}=\sum_{c} N_{a b}^{c} s_{c x}
$$

Comparing this equation with Eq. (221), we conclude that

$$
\frac{s_{a x}}{s_{1 x}}=\lambda_{j}(a) \quad \text { for some } j=j(x)
$$

In other words, for each $x$ the map $a \mapsto s_{a x} / s_{1 x}$ is a fusion character.

In the next section we will see that if braiding is sufficiently nontrivial, then the $S$-matrix is unitary. In this case, the map from labels to fusion characters is one-to-one, and Eq. (226) may be cast into a form known as Verlinde formula:

$$
N_{a b}^{c}=\sum_{x} \frac{s_{a x} s_{b x} s_{\bar{c} x}}{s_{1 x}}
$$

\section{E.5 Braiding nondegeneracy $=$ modularity}

Let us recall the definition of a superselection sector: it is a class of states that can be transformed one to another by local operators. From the physical perspective, the operators we use must respect any unbroken symmetry present in the Hamiltonian, e.g., the U(1) symmetry associated with the conservation of electric charge. Another example is the number of fermions 
modulo 2, if that number is not conserved as an integer. (Here we speak about actual fermions forming the system rather than effective Majorana modes obtained by a nonlocal transformation.) However, in the model studied in this paper the superselection sectors are stable with respect to all local operators. More generally, we may consider an arbitrary system built of spins or other bosonic degrees of freedom, and ask for properties that are stable to a generic perturbation. In this case, the superselection sectors have purely topological nature: some signature of a nontrivial excitation $x \neq 1$ will be preserved even if we cut out a piece of material containing the quasiparticle. It is quite reasonable to assume that the presence of such an excitation can actually be detected by an Aharonov-Bohm measurement, i.e., by moving a test particle $a$ around $x$. Thus we arrive at the condition that the braiding is nondegenerate, which must be true for any anyonic system not complicated by external symmetries.

Definition E.11. Braiding is said to be nondegenerate if for each label $x \neq 1$ there is some label $a$ such that the operator $R_{a x} R_{x a}$ is not identity.

Theorem E.12. In a theory with nondegenerate braiding, the following operator $S_{z}$ acting in the space $\mathcal{L}_{z}=\bigoplus_{b} V_{b}^{b z}$ is unitary:

$$
S_{z} \bigvee_{b}^{b} \psi \stackrel{\text { def }}{=} \frac{1}{\mathcal{D}} \sum_{a} d_{a} \bigcap_{a}^{a} \int_{b}^{b} \psi, \quad \text { where } \psi \in V_{b}^{b z}
$$

Note that the standard $S$-matrix corresponds to $z=1$. The operator $S_{z}^{\dagger}$ differs from $S_{z}$ by the orientation of crossings. (This is easy to show by considering a matrix element of $S_{z}$.) The converse of Theorem E.12 is also true and can be proved easily: if the standard $S$-matrix is unitary, then the braiding is nondegenerate.

Lemma E.13. The operator $R_{a x} R_{x a}$ is trivial for all labels a if and only if $x$ is mapped to the trivial fusion character $j(x)=j(1)$, i.e., if $s_{a x} / s_{1 x}=d_{a}$ for all a.

Proof. The triviality of $R_{a x} R_{x a}$ means that for all $c, R_{c}^{a x} R_{c}^{x a}=\mathrm{id}_{c}^{x a}$, i.e., $\frac{\theta_{c}}{\theta_{x} \theta_{a}}=1$ whenever $N_{x a}^{c} \neq 0$. We calculate $s_{x \bar{a}}=s_{a x}^{*}$ using Eq. (223):

$$
\mathcal{D} s_{x \bar{a}}=\sum_{c} N_{x a}^{c} \frac{\theta_{c}}{\theta_{x} \theta_{a}} d_{c}=\sum_{c} N_{x a}^{c} d_{c}=d_{x} d_{a} .
$$

Thus, $s_{a x} / s_{1 x}=d_{a}$. Conversely, if the operator $R_{a x} R_{x a}$ is nontrivial, then

$$
\mathcal{D} \operatorname{Re} s_{x \bar{a}}=\sum_{c} N_{x a}^{c} \operatorname{Re}\left(\frac{\theta_{c}}{\theta_{x} \theta_{a}}\right) d_{c}<\sum_{c} N_{x a}^{c} d_{c}=d_{x} d_{a},
$$

therefore $s_{a x} / s_{1 x} \neq d_{a}$.

The proof of Theorem E.12 is based on the following equation, which is useful on its own right:

$$
\frac{1}{\mathcal{D}^{2}} \sum_{a} d_{a} \bigodot_{x}^{x} a=\left.\delta_{j(x), j(1)}\right|_{x} ^{x}
$$


Indeed, the condition $j(x)=j(1)$ means that the fusion character $\lambda_{j(x)}: a \mapsto s_{a x} / s_{1 x}$ is trivial, i.e., $s_{a x} / s_{1 x}=d_{a}$. On the other hand, if $\lambda_{j(x)}$ is nontrivial, then it is orthogonal to the trivial character, i.e., $\sum_{a} d_{a} s_{a x}^{*}=0$. The above equation combines both cases.

Proof of Theorem E.12, By Lemma E.13, the nondegeneracy condition implies that $j(x)=$ $j(1)$ if and only if $x=1$. Thus, the linear combination of loops in Eq. (230) is a projector onto label 1 . We apply it as follows:

$$
\begin{aligned}
& \frac{1}{\mathcal{D}^{2}} \sum_{a} d_{a}\left(\sum_{\substack{\mid \\
y}}^{x}\right)^{y} a=\frac{1}{d_{x}} \delta_{\bar{x} y} \bigodot_{x}^{x} \\
& S_{z}^{\dagger} S_{z} \psi=\frac{1}{\mathcal{D}^{2}} \sum_{x, a} d_{x} d_{a} \bigcap_{x}^{x} \int_{b}^{b} \psi=\sum_{x}^{z} d_{x} \frac{\delta_{x b}}{d_{x}} \bigodot_{x}^{x} \psi=\psi .
\end{aligned}
$$

Theories with a unitary $S$-matrix are often called modular due to their relation to the group of diffeomorphisms of the two-dimensional torus considered up to topological equivalence (namely, isotopy). Let us discuss this subject at a speculative level. The first thing to note is that a system of spins or electrons can in principle be put on a torus whether or not the braiding is nondegenerate. However, as indicated earlier, degeneracy generally occurs due to some external symmetry. If it is a gauge symmetry, then putting the system on the torus involves some choice. For example, in the case of U(1) symmetry, one may run an arbitrary magnetic flux through each basic circle of the torus. Those fluxes can be detected by Aharonov-Bohm measurements, but they cannot be changed by intrinsic operations, which include splitting, fusion, and moving particles around the torus. Mathematically, such operations form a so-called skein algebra, which may be defined in terms of graphs on the torus up to equivalence transformations. (Recall that a planar graph without external lines can be transformed to a number, therefore the skein algebra of the plane is simply $\mathbb{C}$.) The skein algebra is a finite-dimensional $C^{*}$-algebra; in general it is a direct sum of several blocks, each block being isomorphic to the algebra of operators on some finite-dimensional Hilbert space. Physically, different blocks correspond to different values of external parameters such as the magnetic fluxes. Modular theories are special in that there is only one block. In other words, the torus is characterized by a single finite-dimensional space.

The Hilbert space of the torus is actually $\mathcal{M}=\mathbb{C}^{M}$. A basis in this space may be associated with effective anyonic charge that is detected by an Aharonov-Bohm measurement along some circle. Different circles correspond to different bases. The $S$-matrix effects a transition between two bases. Other important matrices are $C=\left(\delta_{\bar{a} b}: a, b \in M\right)$ (which corresponds to a $180^{\circ}$ degree rotation) and $T=\left(\theta_{a} \delta_{a b}: a, b \in M\right)$ (which corresponds to a Dehn twist). We will justify this description by showing that $S, C$, and $T$ obey the modular relations up to a phase factor $\Theta$ :

$$
(S T)^{3}=\Theta C, \quad S^{2}=C, \quad C^{2}=I,
$$


where

$$
\Theta=\mathcal{D}^{-1} \sum_{a} d_{a}^{2} \theta_{a}
$$

As a corollary, note that $\Theta$ is a root of unity. Indeed, $S^{4}=I$, therefore $\Theta^{4|M|}=(\operatorname{det} T)^{12}$ is a product of topological spins, which are roots of unity by Theorem E.10.

Let us actually study relations between more general operators acting in the space $\mathcal{L}_{z}=$ $\bigoplus_{b} V_{b}^{b z}$ (which corresponds to a punctured torus, with $z$ being the anyonic charge of the puncture). The operator $S_{z}$ has been already defined, whereas

$$
C_{z} \bigvee_{\psi}^{b} \stackrel{\text { def }}{=} \theta_{b}^{*} \bigvee_{\bar{b}}^{\bar{b}} \gamma_{b}^{b} \psi, \quad T_{z} \bigvee_{b}^{b} \stackrel{\text { def }}{=} \theta_{b} \bigvee_{b}^{b}
$$

Theorem E.14. The operators $S_{z}, C_{z}$, and $T_{z}$ satisfy the following modular relations:

$$
S_{z}^{\dagger} T_{z} S_{z}=\Theta T_{z}^{\dagger} S_{z}^{\dagger} T_{z}^{\dagger}, \quad S_{z}=S_{z}^{\dagger} C_{z}, \quad C_{z}^{2}=\theta_{z}^{*} .
$$

Proof. The calculation of $S_{z}^{\dagger} T_{z} S_{z}$ parallels the proof of Theorem E.12, but the nondegeneracy condition is not necessary. First, let us apply the $S$-matrix to the row vector with entries $d_{a} \theta_{a}$ :

$$
\sum_{a} d_{a} \theta_{a} s_{a \bar{x}}=\frac{1}{\mathcal{D}} \sum_{a, c} d_{a} N_{a x}^{c} \frac{\theta_{c}}{\theta_{x}} d_{c}=\frac{1}{\mathcal{D}} \sum_{c} d_{x} d_{c} \frac{\theta_{c}}{\theta_{x}} d_{c}=\Theta d_{x} \theta_{x}^{*}
$$

The result may be written as follows:

$$
\frac{1}{\mathcal{D}} \sum_{a} d_{a} \theta_{a} \bigcup_{x}^{x} a=\Theta \bigodot_{x}^{x}
$$

Then we replace the single line by two lines and simplify the result (cf. Eq. (217)):

$$
\frac{1}{\mathcal{D}} \sum_{a} d_{a} \theta_{a}(\left.\left.\right|_{\substack{\mid \\ y}} ^{x}\right|_{i} ^{y}=\left.\Theta \overbrace{x}\right|_{y} ^{x}=\Theta \theta_{x}^{*} \overbrace{y}^{x} \theta_{y}^{*}
$$

Attaching a graph representing $\psi \in V_{\bar{y}}^{\bar{y} z}$ to the first and the last expression, we get $S_{z}^{\dagger} T_{z} S_{z} \psi$ and $\Theta T_{z}^{\dagger} S_{z}^{\dagger} T_{z}^{\dagger} \psi$, respectively.

Now we show that $S_{z}=S_{z}^{\dagger} C_{z}$. If $\psi \in V_{b}^{b z}$, then

$$
S_{z} \psi=\frac{1}{\mathcal{D}} \sum_{a} d_{a} \bigcap_{a}^{a} \int_{b}^{b} \psi=\frac{1}{\mathcal{D}} \sum_{a} d_{a} \bigcap_{l_{a}}^{a} \bigwedge_{b}^{b} \psi=\frac{1}{\mathcal{D}} \sum_{a} d_{a} \theta_{b}^{*} \bigodot_{a}^{a} \int_{b}^{b} \psi=S_{z}^{\dagger} C_{z} \psi
$$

The formula $C_{z}^{2} \psi=\theta_{z}^{*} \psi$ follows from (217). 


\section{E.6 Gauge freedom and Ocneanu rigidity}

In the preceding sections, we defined a theory of anyons as a solution to a certain system of algebraic equations for $F_{u}^{a b c}, \alpha_{a}, \beta_{a}$, and $R_{c}^{a b}$. Specifically, these are the pentagon equation, the triangle and hexagon equations as well as unitarity conditions. (The unitarity conditions are algebraic in the real and imaginary part of the corresponding matrices.) The set of solutions is some real algebraic variety. This description, however, does not take into account a gauge degree of freedom. Indeed, two solutions are physically equivalent if they are related to each other by a simultaneous basis change in the spaces $V_{c}^{a b}$. In the examples studied in this paper, quotienting over such transformations makes the set of solutions discrete. In other words, the algebraic structure is rigid, with the physical consequence that the properties of anyons are stable to small perturbations of the Hamiltonian. Is it true in general? The affirmative answer was obtained by Ocneanu, but he did not publish his proof. The only written proof I know of is due to Etingof, Nikshych, and Ostrik [30, but it is more general and hence complex; in particular, it does not depend on the unitarity or the pivotal property. Meanwhile, the proof of Ocneanu rigidity for unitary theories is not very difficult and may be interesting to a mathematical physicist.

To prove that a solution to an equation system is rigid, one needs to study infinitesimal deformations. Let us call a deformation of $F, \alpha, \beta, R$ permissible if it satisfies the equations in the first order of Taylor expansion. The deformation is called trivial if it can be obtained by a change of basis, also in the first order. (By restricting our attention to the first order, we potentially make the space of permissible deformations larger and the space of trivial deformation smaller.) Our goal is to calculate the quotient, permissible modulo trivial, and show that it vanishes. The analysis consists of several steps.

First, we only consider $F$ and the pentagon equation. The resulting deformation problem resembles the definition of $H^{3}(G, \mathbb{R})$ for a group $G$. In fact, it is exactly that in example 2 on page 72. Associated with an arbitrary fusion theory is a cochain complex defined by Crane and Yetter [91] and independently by Davydov [92]. It is a sequence of real spaces and linear maps

$$
C^{1} \stackrel{\delta^{1}}{\longrightarrow} C^{2} \stackrel{\delta^{2}}{\longrightarrow} C^{3} \stackrel{\delta^{3}}{\longrightarrow} \cdots
$$

whose third cohomology space $H^{3}(C) \stackrel{\text { def }}{=} \operatorname{Ker} \delta^{3} / \operatorname{Im} \delta^{2}$ classifies the nontrivial deformations of the theory. (The first and the second cohomology spaces have natural interpretation too.) We will use the term "tangent cohomology" 23 proposed by Davydov.

It is well known that any finite group has trivial real cohomology in all dimensions $n \geqslant 1$. Likewise, the tangent cohomology vanishes for an arbitrary unitary fusion theory (actually, any fusion theory). The proof of this statement is based on the same idea as the corresponding proof for groups. In the latter case, one uses averaging over the group, which is well-defined because the group is finite. For a unitary fusion theory, one needs to take the quantum trace (actually, a partial quantum trace) and average it over the label set with weight $d_{a} / \mathcal{D}^{2}$.

The next step is to include $\alpha, \beta$ and to consider the triangle equations. This is just a technical detail that involves trivial changes to the deformation theory [93]. (The argument is quite general and applicable even if the label set is infinite, in which case the cohomology may

\footnotetext{
${ }^{23}$ Etingof, Nikshych, and Ostrik call it "Yetter cohomology" in recognition of Yetter's further work in this area.
} 
not vanish.) The rigidity of braiding follows from the vanishing of $H^{2}(C)$ in conjunction with Vafa's theorem (see Theorem E.10).

E.6.1 Gauge freedom in the description of anyons. An isomorphism between two fusion theories, $\mathcal{A}$ and $\mathcal{A}^{\prime}$ is given by a bijection between the label sets (we simply assume that they are equal) and a collection of unitary maps ${ }^{24} \Gamma_{c}^{a b}:\left(V^{\prime}\right)_{c}^{a b} \rightarrow V_{c}^{a b}$; these data are enough to relate one system of associativity constraints to the other:

$$
F_{u}^{a b c},\left(F^{\prime}\right)_{u}^{a b c}: Y_{e}^{a} Y_{u}^{c} \longrightarrow \bigvee_{u}^{a} Y_{f}^{c}, \quad F_{u}^{a b c}\left(\sum_{e} \Gamma_{e}^{a b} \otimes \Gamma_{u}^{e c}\right)=\left(\sum_{f} \Gamma_{u}^{a f} \otimes \Gamma_{f}^{b c}\right)\left(F^{\prime}\right)_{u}^{a b c} .
$$

For example, let $\mathcal{A}^{\prime}$ be obtained from $\mathcal{A}$ by changing left and right, i.e., $\left(V^{\prime}\right)_{c}^{a b}=V^{b a}$ and $\left(F^{\prime}\right)_{u}^{a b c}=\left(F_{u}^{c b a}\right)^{-1}$. If theory $\mathcal{A}$ has braiding $R$, then $\Gamma_{c}^{a b}=\left(R_{c}^{a b}\right)^{-1}$ is an isomorphism (for a quick demonstration, apply the Yang-Baxter braid (205) to both trees above). However, we will be mostly interested in the case where $\left(V^{\prime}\right)_{c}^{a b}=V_{c}^{a b}$ so that $\Gamma_{c}^{a b}$ may be called "basis change"; examples can be found in Sections 8.4 and 9

By analogy with Eq. (239), one can write similar equations for $\alpha_{a}$ vs. $\alpha_{a}^{\prime}$ and $\beta_{a}$ vs. $\beta_{a}^{\prime}$. However, the physical meaning of $\alpha$ and $\beta$ is rather elusive, so it is not clear whether we obtain the most general form of equivalence this way. A reasonable notion of isomorphism between two theories, namely tensor functor arises naturally in the categorical formalism (see Sec. E.7.3). To state it in concrete terms, we just need to supplement the basis change with an overall phase factor $\gamma$ so that

$$
\alpha_{a}=\Gamma_{a}^{a 1} \gamma \alpha_{a}^{\prime}, \quad \beta_{a}=\Gamma_{a}^{1 a} \gamma \beta_{a}^{\prime} .
$$

Finally, the braiding rules are changed as follows:

$$
R_{c}^{a b} \Gamma_{c}^{a b}=\Gamma_{c}^{b a}\left(R^{\prime}\right)_{c}^{a b}
$$

Thus we have defined the family of theories $\mathcal{A}^{\prime}$ that are isomorphic to a given theory $\mathcal{A}$. However, their parametrization by $\Gamma_{c}^{a b}$ and $\gamma$ is redundant. In particular, $F, \alpha, \beta, R$ do not change if we replace $\Gamma_{c}^{a b}$ and $\gamma$ with $\Phi_{c}^{a b}$ and $\varphi$ such that

$$
\Phi_{c}^{a b}=\frac{h_{c}}{h_{a} h_{b}} \Gamma_{c}^{a b}, \quad \varphi=h_{1} \gamma,
$$

where $h_{x}$ are arbitrary phase factors. In the categorical language, the tensor functors defined by $(\Gamma, \gamma)$ and by $(\Phi, \varphi)$ are isomorphic.

Thus we have entities of dimension 0,1 , and 2: theories of anyons, 1-isomorphisms (i.e., isomorphisms between theories), and 2-isomorphisms (i.e., isomorphisms between 1-isomorphisms). Let us define three sets indexed by a complementary dimension:

1. 2-automorphisms $h$ of a given 1-isomorphism $(\Gamma, \gamma)$;

2. Equivalence classes of 1 -automorphisms $(\Gamma, \gamma)$ of a given theory $\mathcal{A}$ up to 2 -isomorphisms;

3. Equivalence classes of theories with given fusion multiplicities up to 1-isomorphisms.

The first set is an Abelian group, the second is a group (see Appendix $\mathbb{F}$ ), the third does not have any special structure.

\footnotetext{
${ }^{24}$ The direction of these maps is chosen to be consistent with the definition of a tensor functor (see Definition E.25.
} 
E.6.2 Infinitesimal deformations and the vanishing of tangent cohomology. Let us define infinitesimal analogues of sets 1,2, 3; we will eventually prove that they are trivial. The constructions involved are typical to cohomology theory, following a pattern that may already be recognized by studying set 1 . We are eventually interested in set 3 , which classifies solutions of the pentagon equation up to a basis change. In this subsection we ignore braiding and all attributes of the vacuum sector (i.e., $\gamma, \alpha, \beta$, and the triangle equations); these things will be considered later.

For a fixed fusion theory with associativity constraints $F_{u}^{a b c}$, let

$$
h_{a} \approx 1-i X_{a}, \quad \Gamma_{c}^{a b} \approx \operatorname{id}_{V_{c}^{a b}}-i Y_{c}^{a b}, \quad\left(F^{\prime}\right)_{u}^{a b c} \approx F_{u}^{a b c}\left(\operatorname{id}_{V_{u}^{a b c}}-i Z_{u}^{a b c}\right),
$$

where $X_{a}$ is an infinitely small real number and $Y_{c}^{a b}, Z_{u}^{a b c}$ are infinitely small Hermitian operators acting in $V_{c}^{a b}$ and $V_{u}^{a b c}$, respectively. ${ }^{25}$ Now, let us substitute the expressions for $h$ and $\Gamma$ into Eq. (242), assuming that $\Phi_{c}^{a b}=\operatorname{id}_{V_{c}^{a b}}$. We get:

$$
Y_{c}^{a b}=\left(\delta^{1} X\right)_{c}^{a b} \stackrel{\text { def }}{=}\left(X_{b}-X_{c}+X_{a}\right) \operatorname{id}_{V_{c}^{a b}}
$$

An infinitesimal analogue of set 1 is the space of deformations $X=\left(X_{a}: a \in M\right)$ such that $\delta^{1} X=0$. One can deal with Eq. (239) and the pentagon equation in an analogous way.

To present the results in a more convenient form, let us get rid of the lower index $c$. (In a categorical formulation of the theory, it does not appear at all.) Note that the set of operators $Y_{c}^{a b}$ for all $c$ represents the action of a single element $Y_{a b} \in V_{a b}^{a b}$, which may be constructed as follows:

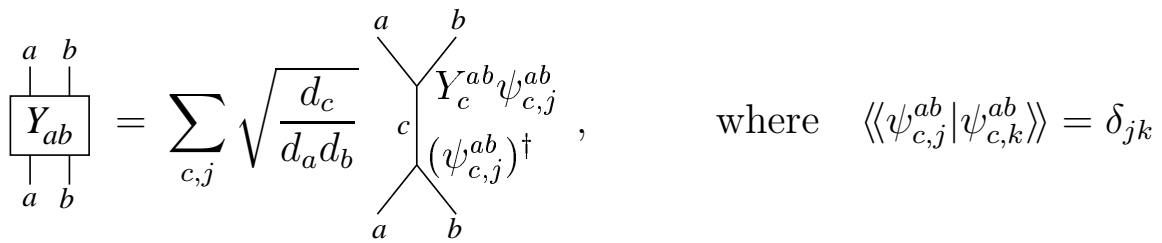

(cf. Eq. 196). For $\left(\delta^{1} X\right)_{c}^{a b}$ this procedure yields:

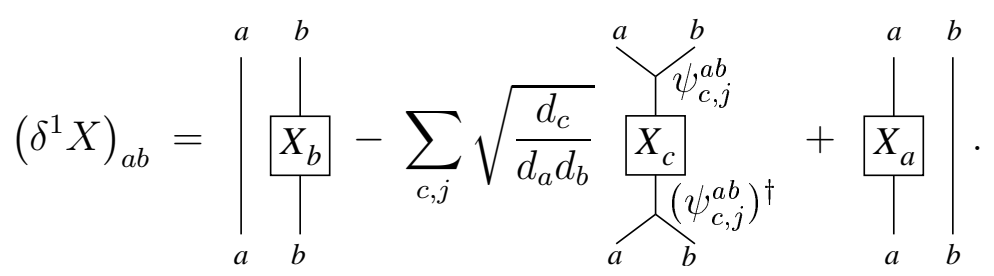

More generally, we can use the following definition.

Definition E.15. Let $C^{n}$ be the set of self-adjoint elements in $\bigoplus_{a_{1}, \ldots, a_{n}} V_{a_{1} \ldots a_{n}}^{a_{1} \ldots a_{n}}$. The tangent complex of the fusion theory is the sequence of $\mathbb{R}$-linear maps

$$
C^{0} \stackrel{\delta^{0}}{\rightarrow} C^{1} \stackrel{\delta^{1}}{\rightarrow} C^{2} \stackrel{\delta^{2}}{\rightarrow} C^{3} \stackrel{\delta^{3}}{\rightarrow} \cdots, \quad \delta^{n}=\sum_{k=0}^{n+1}(-1)^{k} f_{k}^{n}
$$

\footnotetext{
${ }^{25}$ To be rigorous, $Z_{u}^{a b c}$ acts in the space $\bigoplus_{e} V_{e}^{a b} \otimes V_{u}^{e c}$, the representation of $V_{u}^{a b c}$ corresponding to the $((a b) c)$ label grouping. But that is not so important as we always identify different groupings $(=$ trees $)$ using $F$.
} 
where the maps $f_{k}^{n}: C^{n} \rightarrow C^{n+1}$ are defined as follows:

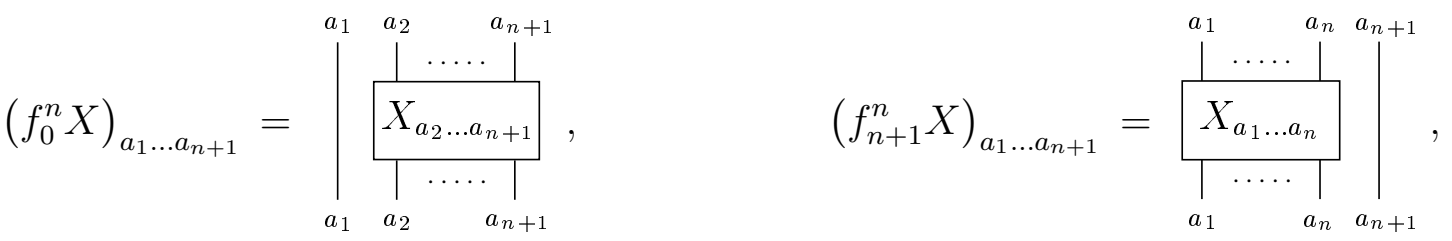

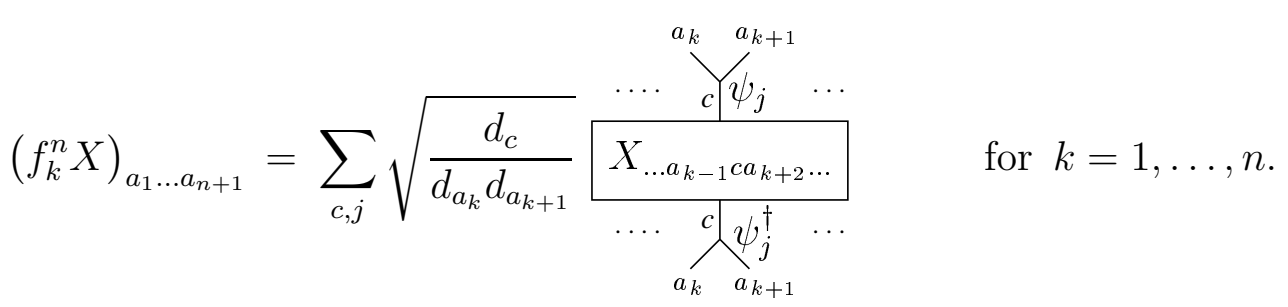

(For $n=0$ we use $V_{\varnothing}^{\varnothing \stackrel{\text { def }}{=}} V_{1}^{1}=\mathbb{C}$, hence $C^{0}=\mathbb{R}$. Correspondingly, $f_{0}^{0}, f_{1}^{0}: 1 \mapsto \sum_{a} \operatorname{id}_{V_{a}^{a}}$, therefore $\delta^{0}=f_{0}^{0}-f_{1}^{0}=0$.)

Note that $C$ is indeed a cochain complex, i.e., $\delta^{n+1} \delta^{n}=0$, which follows from this easily verifiable identity:

$$
f_{k}^{n+1} f_{m}^{n}=f_{m+1}^{n+1} f_{k}^{n} \quad \text { for } 0 \leqslant k \leqslant m \leqslant n+1 .
$$

(It is part of structure that makes $C$ into a cosimplicial space.)

The reader may check that Eq. (239) becomes $Z=\delta^{2} Y$. Therefore the infinitesimal analogue of set 2 is given by solutions to the equation $\delta^{2} Y=0$ modulo elements of the form $Y=\delta^{1} X$. Similarly, the pentagon equation may be written as $\delta^{3} Z=0$, and the solutions should be considered modulo $\delta^{2} Y$. Thus, low-dimensional cohomology of the tangent complex has the following meaning:

- $H^{0}(C)=\mathbb{R}$;

- $H^{1}(C)$ classifies infinitesimal 2-automorphisms of the identity 1-automorphism;

- $H^{2}(C)$ classifies infinitesimal 1-automorphisms (i.e., basis changes that leave the associativity constraints invariant) up to 2-isomorphisms;

- $H^{3}(C)$ classifies infinitesimal deformations of the fusion theory up to 1-isomorphisms (i.e., arbitrary basis changes).

Theorem E.16. $H^{n}(C)=0$ for all $n>0$.

Proof. We will use a standard method of proving vanishing cohomology results, namely contracting homotopy. Let

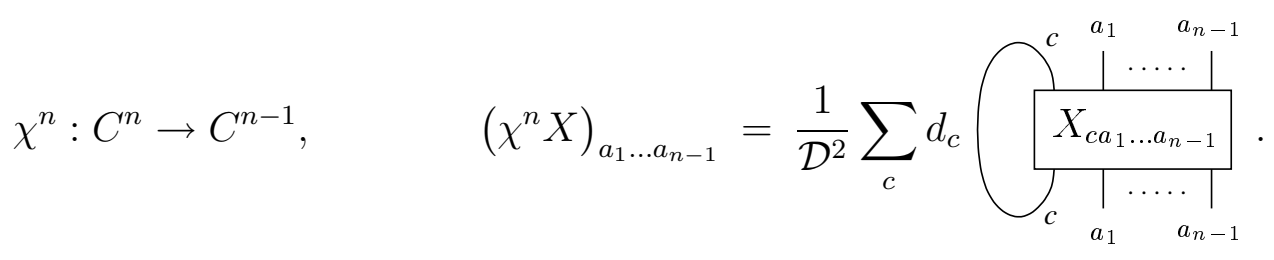


We will show that $\delta \chi+\chi \delta=1$ or, more exactly,

$$
\chi^{n+1} \delta^{n}+\delta^{n-1} \chi^{n}=\operatorname{id}_{C^{n}} \quad \text { for } n>0 .
$$

If this is true, then any $X \in \operatorname{Ker} \delta^{n}$ can be represented as $\delta^{n-1} \chi^{n} X \in \operatorname{Im} \delta^{n-1}$, hence $H^{n}(C)=0$.

Equation (251) is an immediate corollary of this identity:

$$
\chi^{n+1} f_{k}^{n}= \begin{cases}\operatorname{id}_{C^{n}} & \text { if } k=0 \\ f_{k-1}^{n-1} \chi^{n} & \text { if } k, n>0\end{cases}
$$

Let us rewrite it using graphic notation:

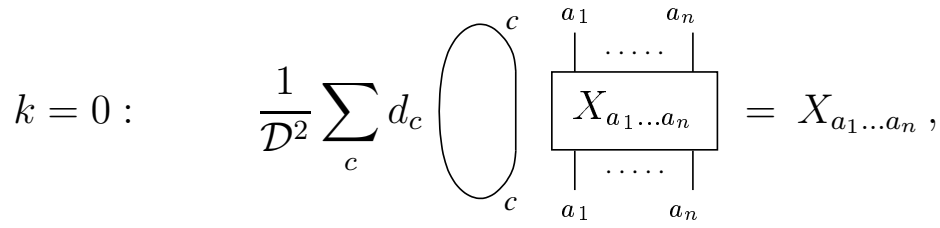

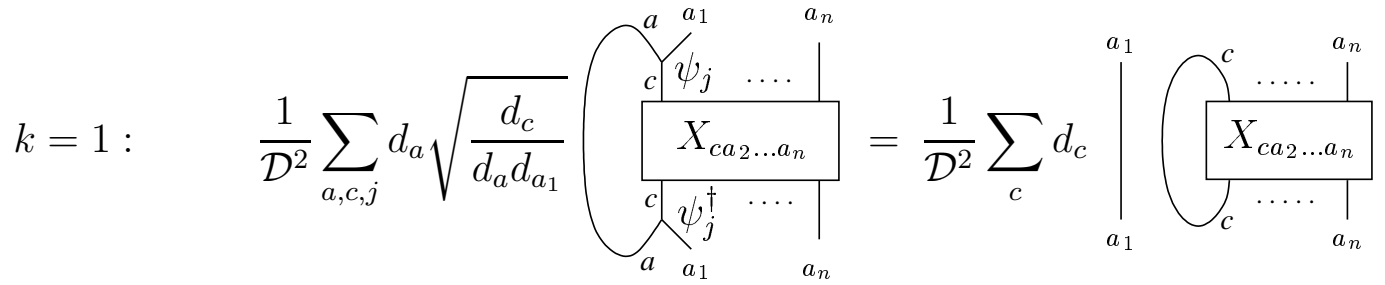

(the case $k>1$ is trivial). The equation for $k=0$ is also obvious, whereas the one for $k=1$ follows from this identity:

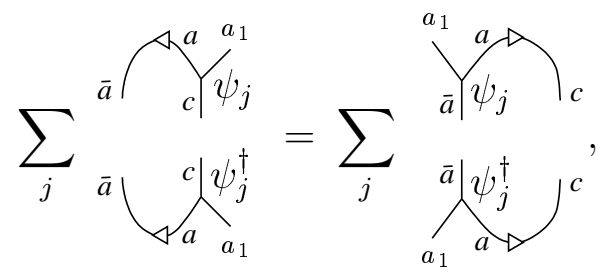

where we have used LemmaE.7. (Note that we did not use the pivotal property or the positivity of the inner product; this sheds some light on why the result holds in a more general setting [30.)

E.6.3 Technicalities related to the unit and braiding. Let us now take into account additional structure that was neglected in the above analysis. We will get more variables and more equations, but the old equations will not change. The new degrees of freedom are characterized by infinitely small real numbers $g, A_{a}, B_{a}$ and Hermitian operators $W_{c}^{a b}$ which are defined as follows:

$$
\begin{aligned}
\gamma \approx 1-i g, \quad \alpha_{a}^{\prime} & \approx\left(1-i A_{a}\right) \alpha_{a}, \quad \beta_{a}^{\prime} \approx\left(1-i B_{a}\right) \beta_{a} \\
\left(R^{\prime}\right)_{c}^{a b} & \approx R_{c}^{a b}\left(\mathrm{id}_{V_{c}^{a b}}-i W_{c}^{a b}\right) .
\end{aligned}
$$


(Recall that $\gamma$ is part of the definition of a 1-isomorphism.) It has been previously shown that any infinitesimal deformation of the associativity constraints $F$ can be compensated by a suitable basis change $\Gamma$. Although $\alpha, \beta$, and $R$ may still remain deformed, the problem is reduced to the case where $F$ is fixed. Furthermore, any infinitesimal basis change not affecting $F$ has the form (242), hence $\alpha, \beta$, or $R$ are not altered either. The only parameter to tune is $\gamma \approx 1-i g$.

Let us first show that any permissible deformation of $\alpha$ and $\beta$ is trivial, where "permissible" means satisfying the triangle equations and "trivial" means satisfying Eq. (240). Taken to an infinitesimal form, the first triangle equation (see Fig. 19a) and Eq. (240) read:

$$
A_{x}=B_{w} \quad \text { for all } x, w ; \quad g+A_{a}=g+B_{a}=0 \quad \text { for all } a .
$$

Clearly, the first condition implies the second if we put $g=-A_{1}$.

Thus we may assume that the whole fusion theory, i.e., $F, \alpha$, and $\beta$ are fixed. It remains to show that the braiding deformation $W$ vanishes, provided it satisfies an infinitesimal version of the hexagon equations. Instead of using the hexagon equations directly, we will rely on the fact that braiding defines an isomorphism between the given fusion theory $\mathcal{A}$ and the theory $\mathcal{A}^{\prime}$ in which left and right are changed. Thus $\left(R_{c}^{a b}\right)^{-1}\left(R^{\prime}\right)_{c}^{a b}$ is an automorphism of $\mathcal{A}$. It follows that $\delta^{2} W=0$, hence

$$
W_{c}^{a b}=\left(\delta^{1} X\right)_{c}^{a b}=\left(X_{b}-X_{c}+X_{a}\right) \operatorname{id}_{V_{c}^{a b}} .
$$

for some $X \in C^{1}$. It is important that the right-hand side is a scalar times the identity, which enables us to calculate the deformation of $R_{c}^{b a} R_{c}^{a b}=\frac{\theta_{c}}{\theta_{a} \theta_{b}}$ easily:

$$
\frac{\theta_{c}^{\prime}}{\theta_{a}^{\prime} \theta_{b}^{\prime}} \approx \frac{\theta_{c}}{\theta_{a} \theta_{b}}\left(1-2 i\left(X_{b}-X_{c}+X_{a}\right)\right) .
$$

But the topological spin is rigid due to Vafa's theorem, hence $X_{b}-X_{c}+X_{a}=0$.

\section{E.7 Categorical formalism (aside)}

Categories and functors are the language used by mathematicians to describe fusion, braiding, and related concepts. I originally tried to write an exposition of the theory of anyons using this formalism, but found it too awkward. I still think that functors are necessary for the understanding of phase transitions and other advanced properties of anyonic systems, but most things can be explained in more elementary terms. This section is a remainder of the abandoned plan. Please be warned that these notes are very incomplete, e.g., there is no discussion of duality and related concepts: rigid, pivotal, and spherical categories (not to mention that we focus on semisimple categories - this restriction is natural for the intended applications).

Let us outline the main elements of the theory. An abstraction called tensor category generalizes the notion of fusion theory. While anyonic fusion has a compact description in terms of basic data, a category is a huge collection of "objects" related by "morphisms". However, these relations form a regular structure that does not leave much freedom of choice. A rather trivial example is the category Hilb, whose objects are all possible finite-dimensional Hilbert spaces and the morphisms are all linear maps. A more interesting category $\operatorname{Rep}(G)$ is defined as follows: the objects are finite-dimensional unitary representations of a compact group $G$ and the morphisms are intertwiners. We may also think of a fusion theory as a category: the objects 
are finite sequences of particle labels, and the morphisms between $\left(b_{1}, \ldots, b_{m}\right)$ and $\left(a_{1}, \ldots, a_{k}\right)$ are fusion/splitting operators, i.e., elements of the space $V_{b_{1} \ldots b_{m}}^{a_{1} \ldots a_{k}}$. Another way to turn a fusion theory into a category will be described later.

By definition, a tensor category is equipped with an operation $\square$ that is analogous to fusion. The role of the vacuum sector is played by a special object 1. In the categories Hilb and $\operatorname{Rep}(G)$, the operation $\square$ is the usual tensor product and $\mathbf{1}$ is the set of complex numbers $\mathbb{C}$ regarded as a one-dimensional space or the trivial representation of $G$.

Yet another example: the matrix category $M a t_{m, m}$. In this category, ${ }^{26}$ objects are $m \times m$ matrices whose entries are finite-dimensional complex linear spaces, morphisms between matrices are entrywise linear maps, and

$$
(A \square B)_{j l}=\bigoplus_{k} A_{j k} \otimes B_{k l}, \quad \mathbf{1}_{j k}= \begin{cases}\mathbb{C} & \text { if } j=k, \\ 0 & \text { if } j \neq k .\end{cases}
$$

(If the matrix entries are finite-dimensional Hilbert spaces, we use the notation $M a t_{m, m}^{\dagger}$ because in this case each morphism has a Hermitian adjoint.) Note that ( $A \square B) \square C=A \square(B \square C)$ and $A \square \mathbf{1}=A=\mathbf{1} \square A$; tensor categories with this property are called strict. In general, the equalities are replaced by isomorphisms $F, \alpha$, and $\beta$ satisfying the pentagon and triangle equations.

A tensor functor is a map from one tensor category to another, a classic example being the embedding $\operatorname{Rep}(G) \rightarrow$ Hilb. Tensor functors have some physical applications, such as transformations of particles by global symmetries (see Appendix $\mathrm{F}$ ). They are also related to the gauge freedom in the description of anyonic fusion, cf. Eqs. (239), (240), and (242). However, our main goal is to understand the status of the isomorphisms $F, \alpha, \beta$. To this end, we will find an embedding of an arbitrary tensor category into a strict one by a tensor functor that preserves distinction between morphisms (such functors are called faithful). In particular, any unitary fusion theory with label set $M$ embeds into the matrix category $M a t_{M, M}^{\dagger}$. The embedding theorem implies

MacLane's coherence theorem: All morphisms composed of $F, \alpha, \beta$ and having the same source and target are equal.

Indeed, in the strict category the source and the target are the same object, and any composition of $F, \alpha$, and $\beta$ is the identity morphism (i.e., equality). Thus all such compositions in the original category are mapped to the same morphism. Since the functor preserves distinction between morphisms, the original morphisms are also equal.

E.7.1 The basics of category theory. A category is a collection of objects and morphisms. The set of morphisms from $A$ to $B$ in a category $\mathcal{C}$ is denoted by $\mathcal{C}(A, B)$.

Morphisms are anything that can be composed: if $f: A \rightarrow B$ and $g: B \rightarrow C$, then $g f: A \rightarrow C$. It is only required that $(h g) f=h(g f)$ and that every object $A$ has an identity morphism $\operatorname{id}_{A}$ such that $f \operatorname{id}_{A}=\operatorname{id}_{B} f=f$ for any $f: A \rightarrow B$. (Example: objects are vertices of a given graph, morphisms are paths of arbitrary length, identity morphisms are paths of length 0.)

\footnotetext{
${ }^{26}$ The more scientific name for this creature is $\mathbb{C}^{m}$-mod- $\mathbb{C}^{m}$, the category of finite-dimensional bimodules over the algebra $\mathbb{C}^{m}$ (the product of $m$ copies of $\mathbb{C}$ ).
} 
A morphism $f: A \rightarrow B$ is called an isomorphism if there is a morphism $f^{-1}: B \rightarrow A$ such that $f^{-1} f=\mathrm{id}_{A}$ and $f f^{-1}=\mathrm{id}_{B}$. Notation for isomorphic objects: $A \cong B$.

For applications in quantum mechanics, the basic example is the category Hilb of finitedimensional Hilbert spaces. Among its objects are the set of complex numbers $\mathbb{C}$ and the onedimensional space corresponding to the first level of a harmonic oscillator. These two spaces are isomorphic but different. (The isomorphism maps the complex number 1 to a normalized wavefunction $\psi$, but this map is not canonical because there is no reason to prefer $\psi$ over $-\psi$.) So, it does not generally make sense to ask whether two given objects are equal (unless they are the same by definition). Morphisms between two given objects may be compared for equality though. For two objects, $X$ and $Y$, a reasonable question is whether $X \cong Y$. Of course, two spaces are isomorphic if and only if they have the same dimension. But we also want to keep track of isomorphisms, for they may not commute: a sequence of isomorphisms may result in a nontrivial automorphism $u: X \rightarrow X, u \neq \mathrm{id}_{X}$. (For Hilbert spaces, it is natural to consider unitary isomorphisms.)

The next example has motivation in superselection theory. Let us consider an infinite spin system which is almost in the ground state, with excitations being allowed only in some finite region. Quantum states of such a system are classified by superselection sectors. For each sector $a$ the state belongs to some finite-dimensional Hilbert space $X_{a}$, which depends on specific constraints on the excitations. We are going to consider different sets of constraints (called "objects") and transformations from one object to another (called "morphisms").

Definition E.17. Let $M$ be some set. The category $V e c_{M}^{\dagger}$ is defined as follows.

- An object $X \in V e c_{M}^{\dagger}$ is a collection of finite-dimensional Hilbert spaces $\left(X_{a}: a \in M\right)$, of which only finitely many are nonzero.

- A morphism $f: X \rightarrow Y$ is a collection of linear maps $f_{a}: X_{a} \rightarrow Y_{a}$ (for each $\left.a \in M\right)$. The identity morphism $\operatorname{id}_{X}: X \rightarrow X$ consists of the unit operators acting in $X_{a}$.

- Additional structure: The set $\operatorname{Vec}_{M}^{\dagger}(X, Y)$ of morphisms from $X$ to $Y$ is a complex linear space. For any $f: X \rightarrow Y$ we define the adjoint morphism $f^{\dagger}: Y \rightarrow X$ such that $\left(f^{\dagger}\right)_{a}=\left(f_{a}\right)^{\dagger}$.

A unitary isomorphism is an isomorphism $f$ such that $f^{-1}=f^{\dagger}$.

An simplified version of this construction is the category $V e c_{M}$ : we use complex linear spaces instead of Hilbert spaces and do not consider adjoint morphisms.

For each $a \in M$ we define the object $[a]$ such that $[a]_{a}=\mathbb{C}$ and $[a]_{b}=0$ for $b \neq a$. Objects isomorphic to $[a]$ are called simple. For any object $Y$,

$$
Y_{a}=\operatorname{Vec}_{M}^{\dagger}([a], Y) .
$$

From the physical perspective, simple objects correspond to quantum states (e.g., a particle pinned to a point), whereas $[a]$ is a reference state in the given superselection sector. For a more formal example, consider the category $\operatorname{Rep}(G)$ whose objects are finite-dimensional unitary representations of a compact group $G$ and whose morphisms are intertwiners. Let $M$ be the set of irreps considered up to isomorphism, and let $[a]$ be a particular irrep in the given isomorphism class. Then we may identify the category $\mathcal{A}=\operatorname{Rep}(G)$ with $V e c_{M}^{\dagger}$ this way: $Y_{a} \stackrel{\text { def }}{=} \mathcal{A}([a], Y)$. 
Remark E.18. Our definition of $V e c_{M}$ and $V e c_{M}^{\dagger}$ resembles the construction of a vector space using coordinates. An invariant characterization is also possible, though somewhat complicated. When the label set $M$ is unspecified, $V e c_{M}$ is called a semisimple $\mathbb{C}$-linear category, and $V e c_{M}^{\dagger}$ is called a unitary category.

The concepts of functor and natural morphism are central in category theory. They are extremely general and therefore hard to grasp. We will try to illustrate them by simple examples, which are still rather abstract. More meaningful (though less direct) examples can be found in the next subsection.

Definition E.19. A functor $\mathcal{F}: \mathcal{A} \rightarrow \mathcal{B}$ maps each object $A$ of the category $\mathcal{A}$ to an object $\mathcal{F}(A)$ of the category $\mathcal{B}$ and each morphism $f: A \rightarrow A^{\prime}$ to a morphism $\mathcal{F}(f): \mathcal{F}(A) \rightarrow \mathcal{F}\left(A^{\prime}\right)$ such that

$$
\mathcal{F}\left(f_{2} f_{1}\right)=\mathcal{F}\left(f_{2}\right) \mathcal{F}\left(f_{1}\right), \quad \mathcal{F}\left(\operatorname{id}_{A}\right)=\operatorname{id}_{\mathcal{F}(A)} .
$$

A morphism (or natural transformation) between two functors $\mathcal{F}, \mathcal{G}: \mathcal{A} \rightarrow \mathcal{B}$ is a collection $h$ of morphisms $h_{A}: \mathcal{F}(A) \rightarrow \mathcal{G}(A)$ such that for any $f: A \rightarrow A^{\prime}$ this diagram commutes

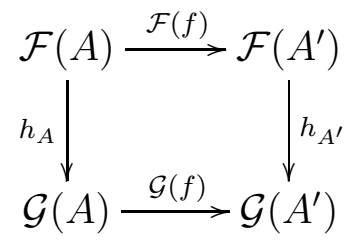

We have already mentioned one example of a functor, namely the embedding $\mathcal{F}: \operatorname{Rep}(G) \rightarrow$ Hilb. Acting on morphisms, it maps the set of intertwiners to the set of all linear maps between two representation spaces. A natural morphism $h: \mathcal{F} \rightarrow \mathcal{F}$ may be constructed as follows: $h_{A}$ is the action of some fixed group element on the representation space of $A$. Indeed, for this particular choice of the functors $\mathcal{F}=\mathcal{G}$ the commutative diagram (258) simply says that $\mathcal{F}(f)$ is an intertwiner.

Example E.20. Let $H$ be a finite-dimensional Hilbert space. We define a unitary functor $\mathcal{H}: H i l b \rightarrow H i l b$ by tensoring with $H$ on the left (notation: $\mathcal{H}=[H \otimes]$ ):

$$
\mathcal{H}(A) \stackrel{\text { def }}{=} H \otimes A, \quad \mathcal{H}(f) \stackrel{\text { def }}{=} \operatorname{id}_{H} \otimes f .
$$

A linear map $u: H \rightarrow G$ between two spaces defines a natural morphism $U=[u \otimes]$ between the corresponding functors:

$$
U_{A} \stackrel{\text { def }}{=} u \otimes \operatorname{id}_{A} \text {. }
$$

The functor $\mathcal{H}=[H \otimes]$ has some special properties, namely the map $f \mapsto \mathcal{H}(f)$ is linear and $\mathcal{H}\left(f^{\dagger}\right)=(\mathcal{H}(f))^{\dagger}$. Such functors are called unitary.

\section{Proposition E.21.}

1. Any unitary functor $\mathcal{F}:$ Hilb $\rightarrow$ Hilb is naturally isomorphic to the left-tensoring functor $[H \otimes]$ for some space $H$, namely $H=\mathcal{F}(\mathbb{C})$. This isomorphism is unitary.

2. Any natural morphism $U:[G \otimes] \rightarrow[H \otimes]$ has the form $[u \otimes]$, where $u=U_{\mathbb{C}}$. 
Proof. The idea is very simple: any space is a direct sum of one-dimensional spaces, any onedimensional space is isomorphic to $\mathbb{C}$, therefore $\mathcal{F}$ and $U$ are completely characterized by their action on $\mathbb{C}$. Let us go through the detail to see how the formalism works.

1. Let $A$ be an arbitrary finite-dimensional Hilbert space. Elements of $A$ may be associated with morphisms $\mathbb{C} \rightarrow A$. An orthonormal basis corresponds to a set of morphisms $\psi_{j}: \mathbb{C} \rightarrow A$ such that $\psi_{k}^{\dagger} \psi_{j}=\delta_{k j} \operatorname{id}_{\mathbb{C}}$ and $\sum_{j} \psi_{j} \psi_{j}^{\dagger}=\operatorname{id}_{A}$. Since $\mathcal{F}$ is a unitary functor,

$$
\mathcal{F}\left(\psi_{k}\right)^{\dagger} \mathcal{F}\left(\psi_{j}\right)=\delta_{k j} \mathrm{id}_{\mathcal{F}(\mathbb{C})}, \quad \sum_{j} \mathcal{F}\left(\psi_{j}\right) \mathcal{F}\left(\psi_{j}\right)^{\dagger}=\operatorname{id}_{\mathcal{F}(A)} .
$$

The same is true for the functor $\mathcal{H}=[\mathcal{F}(\mathbb{C}) \otimes]$. We define a morphism $h_{A}: \mathcal{F}(A) \rightarrow \mathcal{H}(A)$ as follows:

$$
h_{A}=\sum_{j} \mathcal{H}\left(\psi_{j}\right) h_{\mathbb{C}} \mathcal{F}\left(\psi_{j}\right)^{\dagger},
$$

where $h_{\mathbb{C}}: \mathcal{F}(\mathbb{C}) \rightarrow \mathcal{H}(\mathbb{C})=\mathcal{F}(\mathbb{C}) \otimes \mathbb{C}$ is an equality (indeed, $X=X \otimes \mathbb{C}$ for any space $X$ ). It is obvious that $h_{A}^{\dagger} h_{A}=\operatorname{id}_{\mathcal{F}(A)}$ and $h_{A} h_{A}^{\dagger}=\operatorname{id}_{\mathcal{H}(A)}$, hence $h_{A}$ is a unitary isomorphism. To show that $h$ is natural, consider another object $A^{\prime}$ with an orthonormal basis $\left\{\psi_{j}^{\prime}\right\}$ and a linear map $f=\sum_{j, k} c_{j k} \psi_{j}^{\prime} \psi_{k}^{\dagger}$, where $c_{j k} \in \mathbb{C}$. It is easy to check that

$$
h_{A^{\prime}} \mathcal{F}(f)=\sum_{j, k} c_{j k} \mathcal{H}\left(\psi_{j}^{\prime}\right) h_{\mathbb{C}} \mathcal{F}\left(\psi_{k}\right)^{\dagger}=\mathcal{H}(f) h_{A} .
$$

2. Let $\mathcal{G}=[G \otimes]$ and $\mathcal{H}=[H \otimes]$. For an arbitrary object $A$ with an orthonormal basis $\left\{\psi_{j}\right\}$ we have $U_{A} \mathcal{G}\left(\psi_{j}\right)=\mathcal{H}\left(\psi_{j}\right) U_{\mathbb{C}}$ (due to the naturality of $U$ ). Hence

$$
U_{A}=U_{A} \operatorname{id}_{\mathcal{G}(A)}=U_{A}\left(\sum_{j} \mathcal{G}\left(\psi_{j}\right) \mathcal{G}\left(\psi_{j}^{\dagger}\right)\right)=\sum_{j} \mathcal{H}\left(\psi_{j}\right) U_{\mathbb{C}} \mathcal{G}\left(\psi_{j}^{\dagger}\right)=U_{\mathbb{C}} \otimes \operatorname{id}_{A} .
$$

Adding another level of abstraction, we can reformulate Proposition E.21 as follows: The category Fun ${ }^{\dagger}$ (Hilb, Hilb) of unitary functors from Hilb to Hilb is isomorphic to the category Hilb. (One may replace Hilb by the category of finite-dimensional complex linear spaces, omitting the unitarity condition.) By analogy, we obtain the following result.

Proposition E.22. The category $F u n^{\dagger}\left(\operatorname{Vec}_{M}^{\dagger}, \operatorname{Vec}_{N}^{\dagger}\right)$ is isomorphic to the category Mat $\dagger_{N, M}^{\dagger}$ whose objects are $N \times M$ matrices of finite-dimensional Hilbert spaces with a finite number of nonzero entries in each column and whose morphisms are entrywise linear maps between such matrices. (If $M$ is finite, then $M a t_{N, M}^{\dagger} \cong \operatorname{Vec}_{N \times M}^{\dagger}$.)

E.7.2 Fusion in categorical terms. Fusion theory may be formulated as additional structure on the category $\mathcal{C}=V e c_{M}^{\dagger}$. Let us assume that the the label set $M$ contains a special element 1 and that $V_{c}^{a b}, F_{u}^{a b c}, \alpha_{a}, \beta_{a}$ are defined and satisfy the pentagon and triangle equations as well as unitarity conditions. If in addition $M$ is finite and the duality axiom on page [74 holds, then the resulting construction is called unitary fusion category. (For a general definition of fusion category see Ref. [30.) 
The use of categorical formalism has mathematical as well as physical motivation. Let $A$ and $B$ be spatially confined excitations such that $A$ is located on the left of $B$. Each excitation may be described by an object in the category $\mathcal{C}$. If we consider both excitations together, we will obtain a new object, $A \square B$, which may be called "physical tensor product". If $A$ and $B$ do not have local degrees of freedom, i.e., $A \cong[a], B \cong[b]$, then $(A \square B)_{c} \cong V_{c}^{a b}$. A nice property of the physical tensor product is that $(A \square B) \square C=A \square(B \square C)$ (provided $A, B$, and $C$ are arranged on the line in that particular order). Mathematically, the operation $\square$ is only associative up to a canonical isomorphism, but we will find some abstract representation of objects in which the isomorphism becomes an equality.

The tensor product of two objects, $A, B \in \mathcal{C}$ is defined by the equation

$$
(A \square B)_{c}=\bigoplus_{a, b} V_{c}^{a b} \otimes A_{a} \otimes B_{b} .
$$

This operation is neither commutative nor a priory associative. Different ways to multiply several objects may be described by parenthesis structures, or trees. For example,

$$
y_{u}^{a} y^{c}: \quad(([a] \square[b]) \square[c])_{u}=\bigoplus_{e} V_{e}^{a b} \otimes V_{u}^{e c} .
$$

The operation $\square$ is a functor, meaning that for any morphisms $f: A \rightarrow A^{\prime}$ and $g: B \rightarrow B^{\prime}$ there is a morphism $f \square g: A \square B \rightarrow A^{\prime} \square B^{\prime}$ such that

$$
\left(f_{2} f_{1}\right) \square\left(g_{2} g_{1}\right)=\left(f_{2} \square g_{2}\right)\left(f_{1} \square g_{1}\right), \quad \operatorname{id}_{A} \square \operatorname{id}_{B}=\operatorname{id}_{A \square B} .
$$

(The tensor product of morphisms is defined in the obvious way: $(f \square g)_{c}=\sum_{a, b} \operatorname{id}_{V_{c}^{a b}} \otimes f_{a} \otimes g_{b}$.)

We may define a unitary isomorphism between $(A \square B) \square C$ and $A \square(B \square C)$ :

$$
F_{A, B, C}:(A \square B) \square C \rightarrow A \square(B \square C), \quad\left(F_{A, B, C}\right)_{u}=\sum_{a, b, c} F_{u}^{a b c} \otimes \operatorname{id}_{A_{a}} \otimes \operatorname{id}_{B_{b}} \otimes \operatorname{id}_{C_{c}},
$$

where $F_{u}^{a b c}$ is the associativity map (177). Similarly,

$$
\alpha_{A}: A \rightarrow A \square[1], \quad \quad \beta_{A}: A \rightarrow[1] \square A
$$

(the definition is obvious). It is important that the isomorphisms $F_{A, B, C}, \alpha_{A}, \beta_{A}$ are natural, i.e., for any morphisms $f: A \rightarrow A^{\prime}, g: B \rightarrow B^{\prime}$, and $h: C \rightarrow C^{\prime}$ we have

$$
\begin{gathered}
F_{A^{\prime}, B^{\prime}, C^{\prime}}((f \square g) \square h)=(f \square(g \square h)) F_{A, B, C}, \\
\alpha_{A^{\prime}} f=\left(f \square \mathrm{id}_{[1]}\right) \alpha_{A}, \quad \beta_{A^{\prime}} f=\left(\operatorname{id}_{[1]} \square f\right) \beta_{A} .
\end{gathered}
$$

Proposition E.23. The functor property (260) and the naturality (263) imply all quadrilateral identities of the tree calculus.

Indeed, there are two kinds of such identities. In the example shown in Fig. 17b, one of the commuting moves occurs inside a subtree descending from the other. The inner move plays the role of $f$ in the naturality condition. If both moves occur in disjoint subtrees, one should use the functor property. 
E.7.3 Main definitions. Among things we are going to define, tensor category is just a general formulation of properties of the operation $\square$. Tensor functor is a new concept though. For an elementary but important example, let the label types and fusion spaces be fixed but the associativity constraints vary. Given two sets of associativity constraints, we may ask whether they are equivalent up to a basis change, cf. Eqs. (239) and (240). This type of equivalence is a special case of tensor functor. Note that two basis transformations may differ by trivial factors, cf. Eq. (242); the corresponding tensor functors are said to be naturally isomorphic.

Definition E.24. A tensor category $\hat{\mathcal{C}}$ is a category $\mathcal{C}$ endowed with a functor $\square: \mathcal{C} \times \mathcal{C} \rightarrow \mathcal{C}$, a special object $\mathbf{1} \in \mathcal{C}$, and natural isomorphisms

$$
F_{A, B, C}:(A \square B) \square C \rightarrow A \square(B \square C), \quad \alpha_{A}: A \rightarrow A \square \mathbf{1}, \quad \beta_{A}: A \rightarrow \mathbf{1} \square A
$$

such that the following diagrams (variants of the pentagon equation in Fig. 17 and the triangle equation in Fig. [19]) commute:
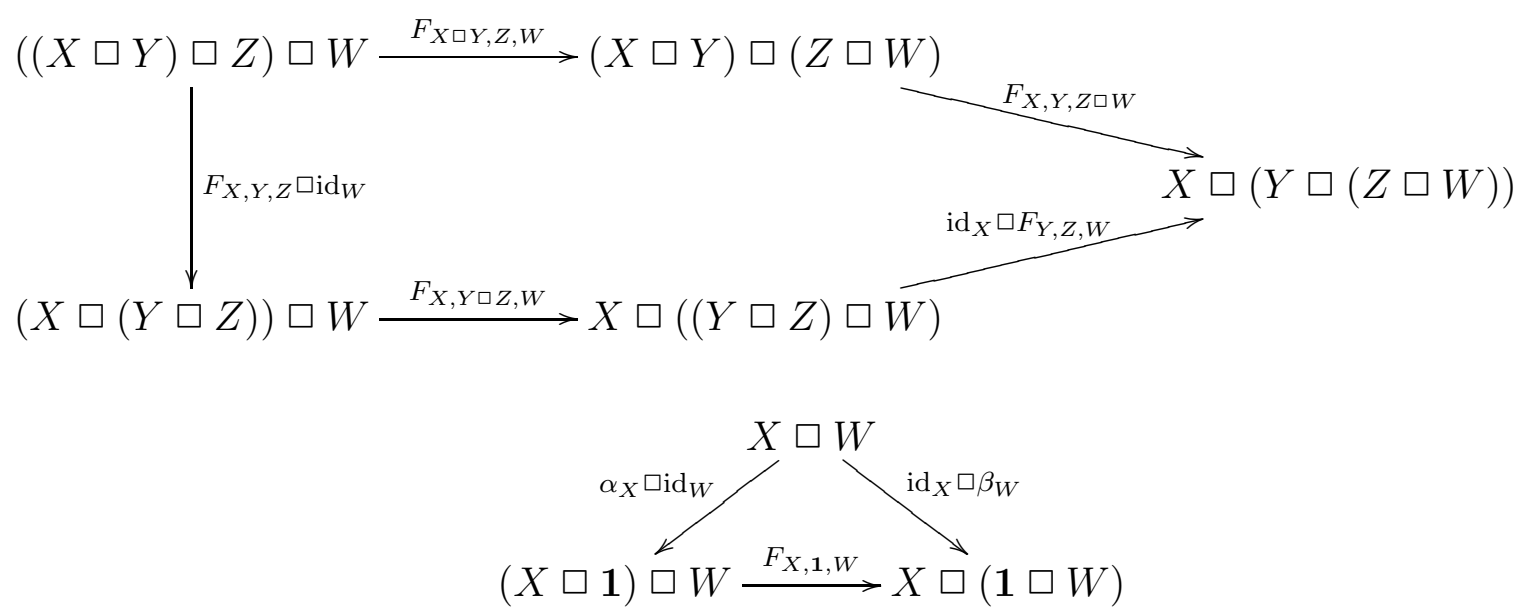

The tensor category $\hat{\mathcal{C}}$ is called strict if $F_{A, B, C}, \alpha_{A}, \beta_{A}$ are equalities. It is called semisimple if the base category $\mathcal{C}$ is $V e c_{M}$. It is called unitary if $\mathcal{C}=V e c_{M}^{\dagger}$, the functor $\square$ is unitary, and the isomorphisms $F, \alpha, \beta$ are unitary.

Semisimple tensor categories admit a compact description in terms of "basic data", which is just slightly more general than that of a fusion theory. Indeed, by analogy with Propositions E.21 and E.22 one can show that any functor of two arguments, $\square: V e c_{M} \times V e c_{M} \rightarrow V e c_{M}$ is given by a set of finite-dimensional spaces $V_{c}^{a b}$. However, the unit object is not necessarily simple, an example being the category $M a t_{M, M}$ or $M a t_{M, M}^{\dagger}$ with the multiplication rule and the unit (256) . (Note that these tensor categories are strict.) In physical terms, the nonsimplicity of the unit means that the vacuum is not unique; thus simple objects in $M a t_{M, M}^{\dagger}$ are boundaries between vacua.

Definition E.25. Let $\hat{\mathcal{C}}=(\mathcal{C}, \square, \mathbf{1}, F, \alpha, \beta)$ and $\hat{\mathcal{C}}^{\prime}=\left(\mathcal{C}^{\prime}, \square^{\prime}, \mathbf{1}^{\prime}, F^{\prime}, \alpha^{\prime}, \beta^{\prime}\right)$ be tensor categories. A tensor functor $\hat{\mathcal{G}}: \hat{\mathcal{C}} \rightarrow \hat{\mathcal{C}}^{\prime}$ is a functor $\mathcal{G}$ between the corresponding base categories, plus two natural isomorphisms, ${ }^{27}$

$$
\Gamma_{A, B}: \mathcal{G}(A) \square^{\prime} \mathcal{G}(B) \rightarrow \mathcal{G}(A \square B), \quad \gamma: \mathbf{1}^{\prime} \rightarrow \mathcal{G}(\mathbf{1}),
$$

\footnotetext{
${ }^{27}$ The direction of arrows is a convention, which is slightly inconvenient for our purposes.
} 
such that the the following diagrams commute:

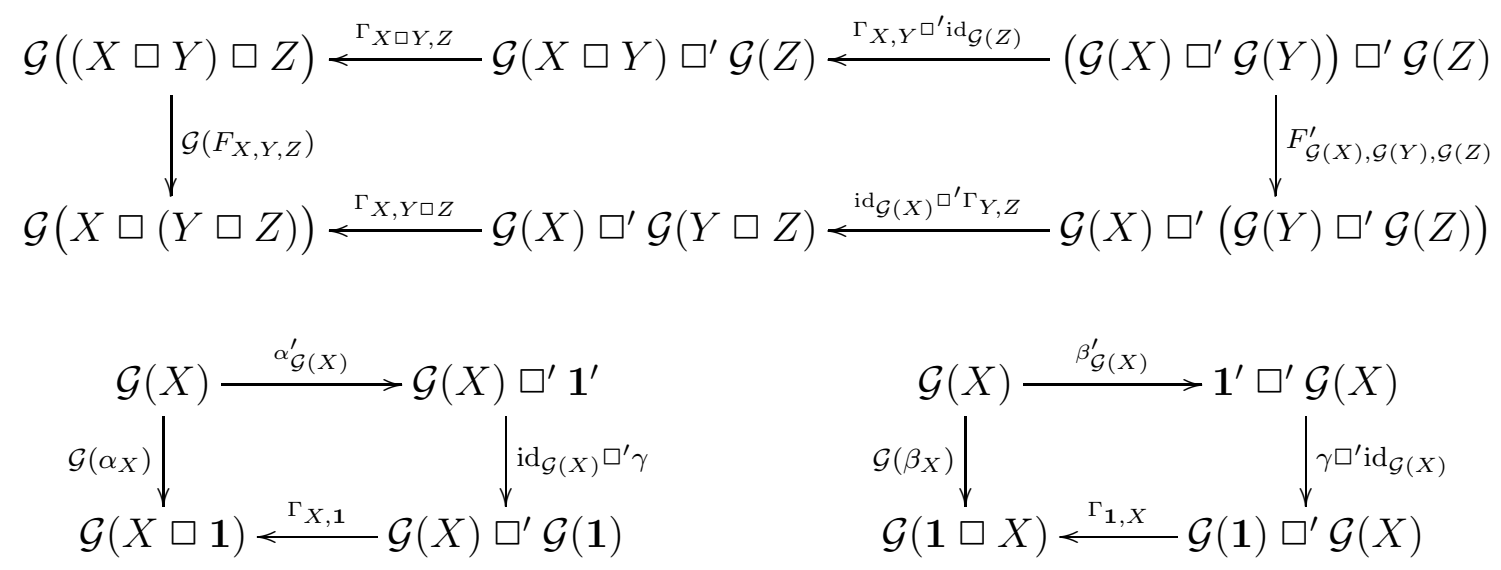

The tensor functor $\hat{\mathcal{G}}=(\mathcal{G}, \Gamma, \gamma)$ is called unitary if all three of its components are unitary.

This definition seems complicated, but basically it says the following: we may identify $\mathcal{G}\left(A \square B\right.$ ) with $\mathcal{G}(A) \square^{\prime} \mathcal{G}(B)$ and $\mathcal{G}(\mathbf{1})$ with $\mathbf{1}^{\prime}$ (by means of $\Gamma$ and $\gamma$ ) so that

$$
\mathcal{G}\left(F_{X, Y, Z}\right)=F_{\mathcal{G}(X), \mathcal{G}(Y), \mathcal{G}(Z)}^{\prime}, \quad \mathcal{G}\left(\alpha_{X}\right)=\alpha_{\mathcal{G}(X)}^{\prime}, \quad \mathcal{G}\left(\beta_{X}\right)=\beta_{\mathcal{G}(X)}^{\prime} .
$$

Remark E.26. A lax tensor functor is defined likewise, but $\Gamma_{A, B}$ and $\gamma$ are not required to be isomorphisms. In physics, this construction describes a situation in which theory $\hat{\mathcal{C}}$ is obtained from $\hat{\mathcal{C}}^{\prime}$ as a result of Bose-condensation, cf. note 9 on page 44.

Definition E.27. A morphism between two tensor functors, $\hat{\mathcal{G}}=(\mathcal{G}, \Gamma, \gamma)$ and $\hat{\mathcal{H}}=(\mathcal{H}, \Phi, \varphi)$ is a natural morphism $h: \mathcal{G} \rightarrow \mathcal{H}$ satisfying these additional commutation relations:
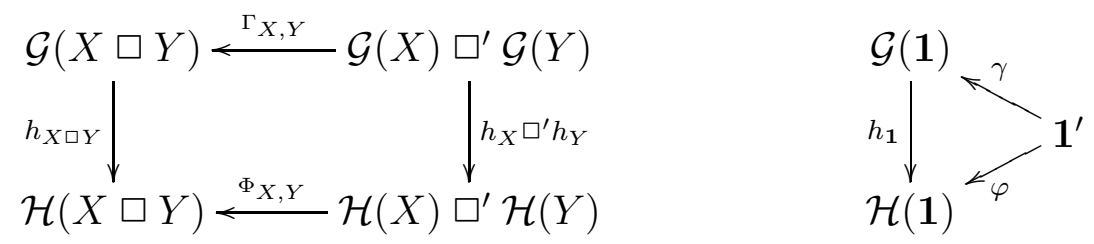

The morphism $h$ is called a (unitary) isomorphism if all the maps $h_{X}$ are (unitary) isomorphisms.

Let us see how these definitions work for fusion theories. Let $\mathcal{C}$ and $\mathcal{C}^{\prime}$ have label sets $M$ and $M^{\prime}$, respectively. We already know that an arbitrary functor $\mathcal{G}: \mathcal{C} \rightarrow \mathcal{C}^{\prime}$ is described by some matrix $\left(G_{a^{\prime}}^{a}: a \in M, a^{\prime} \in M^{\prime}\right)$ of finite-dimensional Hilbert spaces. Then the supplementary components of the tensor functor, $\Gamma$ and $\gamma$ are characterized by some unitary linear maps

$$
\Gamma_{c^{\prime}}^{a b}: \bigoplus_{a^{\prime}, b^{\prime} \in M^{\prime}}\left(V^{\prime}\right)_{c^{\prime}}^{a^{\prime} b^{\prime}} \otimes G_{a^{\prime}}^{a} \otimes G_{b^{\prime}}^{b} \rightarrow \bigoplus_{c \in M} V_{c}^{a b} \otimes G_{c^{\prime}}^{c}, \quad \gamma: \mathbb{C} \rightarrow G_{1^{\prime}}^{1}
$$

Note that since the original $\gamma$ (the map form $\mathbf{1}^{\prime}$ to $\mathcal{G}(\mathbf{1})$ ) is an isomorphism, $G_{a^{\prime}}^{1}=0$ if $a^{\prime} \neq 1^{\prime}$. Eqs. (268) and (269) impose certain algebraic relations on $\Gamma_{c^{\prime}}^{a b}$ and $\gamma$. A unitary isomorphism between two tensor functors is described by a set of unitary maps $h_{a^{\prime}}^{a}: G_{a^{\prime}}^{a} \rightarrow H_{a^{\prime}}^{a}$ satisfying the equations that follow from Eq. (270). 
Returning to the example of basis transformations, let $\mathcal{C}=\mathcal{C}^{\prime}, \square=\square^{\prime}$, but possibly $F \neq F^{\prime}, \alpha^{\prime} \neq \alpha, \beta^{\prime} \neq \beta$. Thus we fix the label types and fusion spaces while allowing different associativity relations. Let us further assume that $\mathcal{G}$ is the identity functor, i.e., $G_{a}^{a}=\mathbb{C}$ and $G_{a^{\prime}}^{a}=0$ if $a^{\prime} \neq a$. In this case, $\Gamma_{c}^{a b}$ is just a unitary operator acting in the space $V_{c}^{a b}=\left(V^{\prime}\right)_{c}^{a b}$, and $\gamma$ is a phase factor. Then Eqs. (268) and (269) become (239) and (240), respectively. In this setting, an isomorphism between two tensor functors is described by unital complex numbers $h_{a}$ satisfying Eq. (242).

E.7.4 The embedding theorem. Now, we will see the "abstract nonsense" in action. While we manipulate with definitions, some combinatorial magic happens behind the scenes. Basically, we turn pentagons into rectangles; this process may be viewed as a proof that the 2-skeleton of the Stasheff polytope is simply connected.

Theorem E.28. For any tensor category $\hat{\mathcal{C}}=(\mathcal{C}, \square, \mathbf{1}, F, \alpha, \beta)$ there exists a strict tensor category $\hat{\mathcal{C}}^{\prime}=\left(\mathcal{C}^{\prime}, \square^{\prime}, \mathbf{1}^{\prime}\right)$ and a faithful tensor functor $\hat{\mathcal{G}}=(G, \Gamma, \gamma)$ from $\hat{\mathcal{C}}$ to $\hat{\mathcal{C}}^{\prime}$ (where "faithful" means that the images of distinct morphisms are also distinct).

Proof (borrowed from Ref. [94]). Let $\mathcal{C}^{\prime}$ be the category of functors from $\mathcal{C}$ to itself, $\square^{\prime}$ the operation of composing two functors (usually denoted by $\circ$ ), and $\mathbf{1}^{\prime}$ the identity functor. We assume that functors act on the left, i.e., $(X \circ Y)(W) \stackrel{\text { def }}{=} X(Y(W))$. For any object $X$ we set $\mathcal{G}(X)=[X \square]$, i.e., $\mathcal{G}(X)(W) \stackrel{\text { def }}{=} X \square W$. The isomorphism $\Gamma_{X, Y}$ between two functors may also be defined in terms of its action on a test object $W$ :

$\Gamma_{X, Y}:[X \square] \circ[Y \square] \rightarrow[(X \square Y) \square], \quad \Gamma_{X, Y}(W) \stackrel{\text { def }}{=} F_{X, Y, W}^{-1}: X \square(Y \square W) \rightarrow(X \square Y) \square W$.

Finally, we define

$$
\gamma: \operatorname{id}_{\mathcal{C}} \rightarrow[\mathbf{1} \square], \quad \gamma(W) \stackrel{\text { def }}{=} \beta_{W}: W \rightarrow \mathbf{1} \square W .
$$

Now we simply rewrite Eqs. (268) and (269), applying them to $W$ :
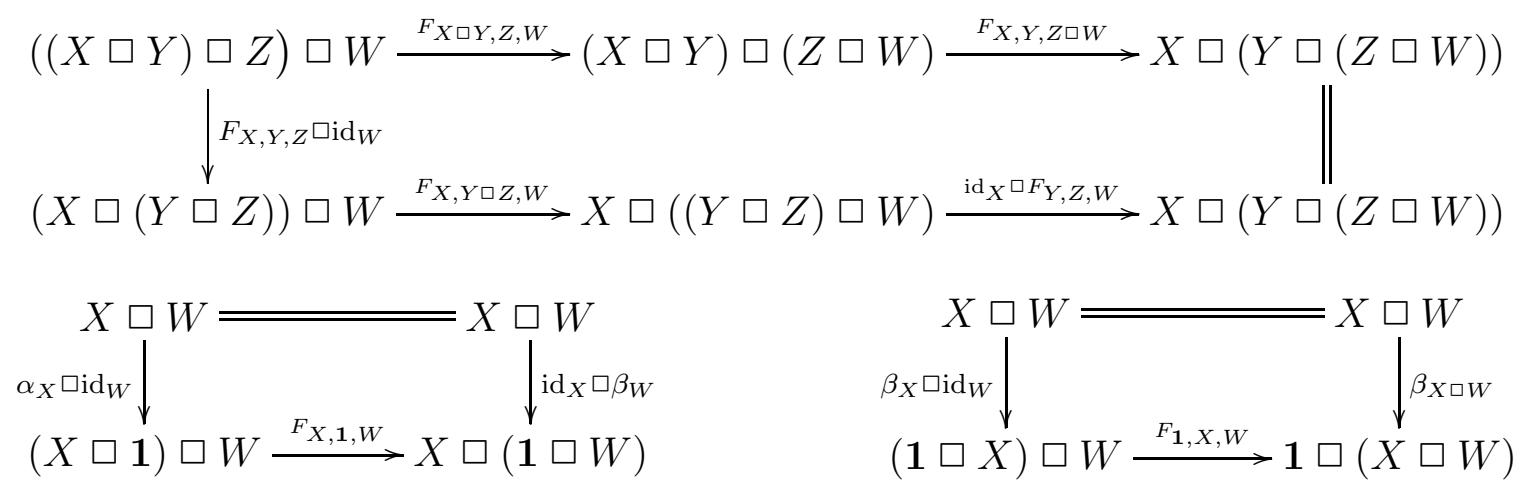

These are the conditions we need to check. But the first and the second of them are identical to Eqs. (264) and (265), respectively. The third condition is a different type of triangle equation, which is shown in Fig. 19] . It follows from the standard one by Lemma E.2.

To see that the functor $\mathcal{G}$ is faithful, we set $W=\mathbf{1}$ and observe that a morphism $h: X \rightarrow Y$ is mapped to $\mathcal{G}(h)(\mathbf{1})=h \square \mathrm{id}_{\mathbf{1}}: X \square \mathbf{1} \rightarrow Y \square \mathbf{1}$. Thus,

$$
\alpha_{Y}^{-1}(\mathcal{G}(h)(\mathbf{1})) \alpha_{X}=h
$$

(due to the naturality of $\alpha$ ). It follows that distinct morphisms remain distinct. 
Note that if $\mathcal{C}=\operatorname{Vec}_{M}^{\dagger}$, then $\mathcal{C}^{\prime}=M a t_{M, M}^{\dagger}$. The functor $\mathcal{G}$ takes a simple object $a$ to the matrix whose $[b, c]$ entry is $V_{c}^{a b}$.

E.7.5 Braiding. Let us be brief and just give some definitions.

Definition E.29. A braiding in a tensor category $\hat{\mathcal{C}}=(\mathcal{C}, \square, \mathbf{1}, F, \alpha, \beta)$ is a collection of isomorphisms

$$
R_{A, B}: A \square B \rightarrow B \square A
$$

which are natural with respect to $A$ and $B$, i.e., for any morphisms $f: A \rightarrow A^{\prime}, g: B \rightarrow B^{\prime}$ this square commutes:

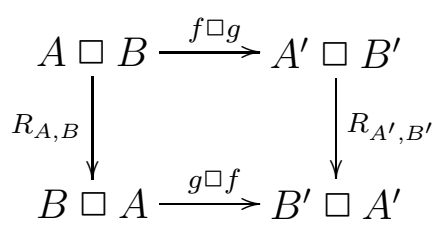

It is also required that the following diagrams are commutative:
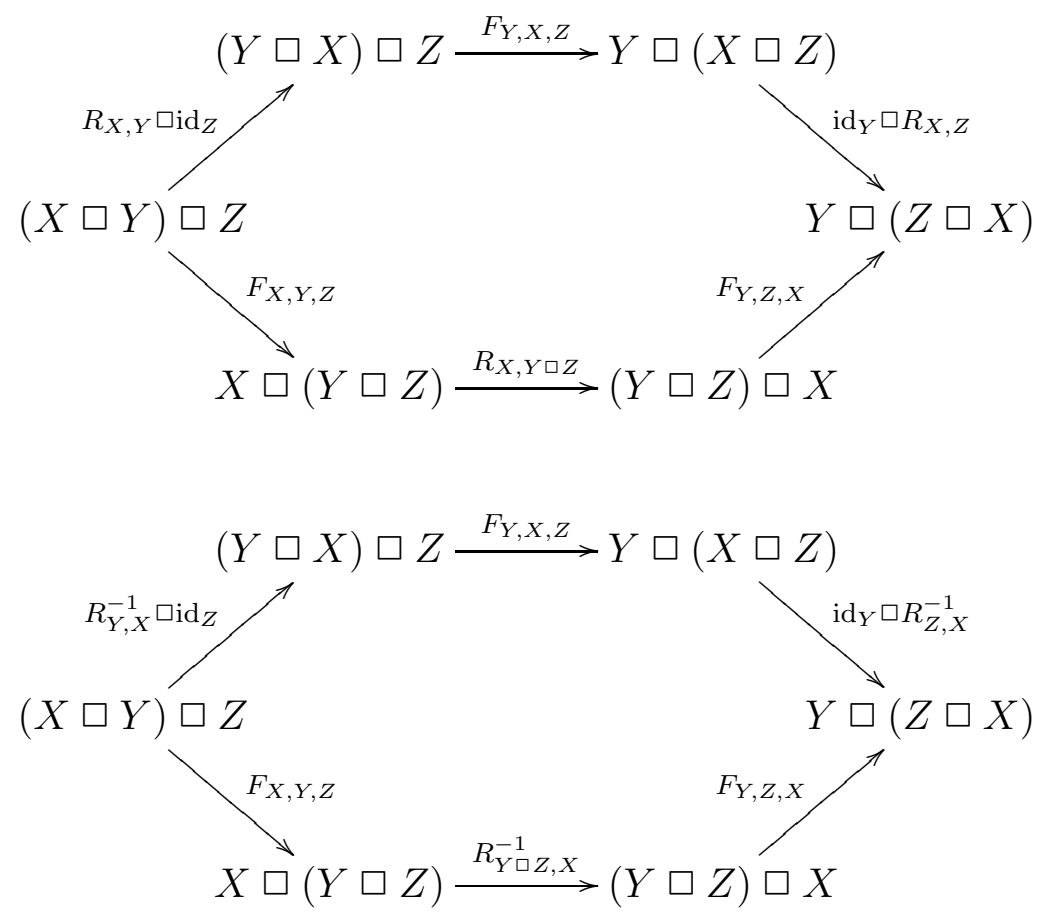

Definition E.30. A braided tensor functor is a tensor functor that commutes with braiding. More exactly, let $\hat{\mathcal{C}}=(\mathcal{C}, \square, \mathbf{1}, F, \alpha, \beta)$ and $\hat{\mathcal{C}}^{\prime}=\left(\mathcal{C}^{\prime}, \square^{\prime}, \mathbf{1}^{\prime}, F^{\prime}, \alpha^{\prime}, \beta^{\prime}\right)$ be tensor categories furnished with braidings $R$ and $R^{\prime}$, respectively. A tensor functor $\hat{\mathcal{G}}=(\mathcal{G}, \Gamma, \gamma)$ from $\hat{\mathcal{C}}$ to $\hat{\mathcal{C}}^{\prime}$ is called braided if the following diagram commutes:

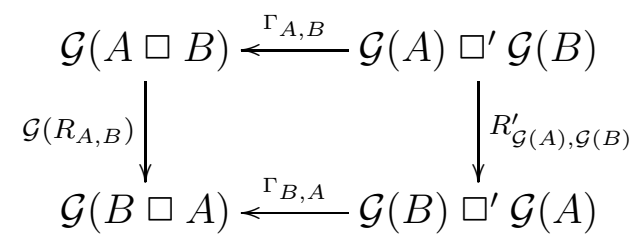


Example E.31. Let $\hat{\mathcal{C}}$ be an arbitrary braided tensor category and let $\hat{\mathcal{C}}^{\prime}$ be defined as follows:

$$
\mathcal{C}^{\prime}=\mathcal{C}, \quad A \square^{\prime} B=B \square A, \quad \mathbf{1}^{\prime}=\mathbf{1}, \quad F_{X, Y, Z}^{\prime}=F_{Z, Y, X}^{-1}, \quad \alpha^{\prime}=\beta, \quad \beta^{\prime}=\alpha, \quad R_{A, B}^{\prime}=R_{B, A} .
$$

Then the identity functor $\mathcal{G}=\mathrm{id}_{\mathcal{C}}$ supplemented with $\Gamma_{A, B}=R_{A, B}^{-1}$ and $\gamma=\mathrm{id}_{\mathbf{1}}$ is a braided tensor functor from $\hat{\mathcal{C}}$ to $\hat{\mathcal{C}}^{\prime}$. (We omit the proof.)

\section{Appendix F: Weak symmetry breaking}

A complete, yet to be discovered description of topological quantum order must include fusion and braiding rules (characterized by a unitary braided fusion category (UBFC), see Appendix E) as well as symmetries of the underlying microscopic Hamiltonian. The no-symmetry case was considered in Sec. E.5. On the other hand, in our study of Abelian anyons in the honeycomb lattice model (see Sec. [5.2) we observed a rather strange property: the translation by a lattice vector interchanges two superselection sectors. This phenomenon may be called a "weak breaking" of the translational symmetry.

Let us now consider an arbitrary two-dimensional many-body system with a symmetry group $G$. For simplicity, we assume that the system consists of spins (or other bosonic degrees of freedom) rather than fermions. There are several ways in which the ground state can break the symmetry that is present in the Hamiltonian. First, the symmetry may be spontaneously broken in the usual sense, i.e., there may be a local order parameter. If such an order parameter does not exist, we may look for finer signs of symmetry breaking, in particular, for nontrivial action of $G$ on superselection sectors. Such an action is described by a homomorphism $\omega_{1}: G \rightarrow \Gamma_{1}$, where $\Gamma_{1}$ is some finite group defined below.

But even if $G$ does not permute the superselection sectors, there is still possibility for subtle symmetry-breaking properties, some of which were studied by Wen [95, 96. under the rubric of "projective symmetry groups" (PSG). While Wen's approach is rather indirect, one may instead investigate the action of symmetry transformations on superselection sectors. For a simple example, let us consider the Hamiltonian (3) with $J_{m}<0$ (note the analogy with Wen's model [96]). The ground state of this Hamiltonian may be obtained from the $J_{m}>0$ state by putting an $m$-particle on every plaquette (so that any missing $m$-particle would now be regarded as an excitation). Therefore an $e$-particle picks up the phase factor -1 when it winds around a plaquette. It follows that the translations by two basis lattice vectors commute up to a minus sign when we consider their action on the nontrivial superselection sector $e$.

Mathematically, the up-to-sign commutation means that the translational group $G=\mathbb{Z} \times \mathbb{Z}$ is replaced by a central extension $G_{e} \rightarrow G$ with kernel $\mathbb{Z}_{2}$. If general, central extensions are classified by the cohomology group $H^{2}(G, \mathrm{U}(1))$. For each superselection sector $a$ we get an extension $G_{a}$. The whole collection of extensions is characterized by a cohomology class $\omega_{2} \in H^{2}\left(G, \Gamma_{2}\right)$, where $\Gamma_{2}$ is some finite Abelian group (see below).

However, this is not the end of the story. Even without anyonic excitations (or if anyons exist but do not complicate the group action in any way), the system may have nontrivial properties such as the integer quantum Hall effect. The latter is described by a Chern-Simons term in the effective action for the electromagnetic field. Dijkgraaf and Witten [97. showed that a general topological action for a gauge field in $2+1$ dimensions is characterized by an element of the cohomology group $H^{4}(B G, \mathbb{Z})$, where $B G$ is the classifying space of $G$. (The 


\begin{tabular}{|c|l|l|}
\hline $\begin{array}{l}\text { Effective dimension } \\
\text { in which the sym- } \\
\text { metry is broken }\end{array}$ & Mathematical description & \multicolumn{1}{|c|}{ Examples } \\
\hline \hline 0 & $\begin{array}{l}\text { A local order parameter taking } \\
\text { values in the coset space } G / H, \\
\text { where } H \text { is a subgroup of } G\end{array}$ & Ferromagnet; Neel phase \\
\hline 1 & $\begin{array}{l}\text { Nontrivial action of the symme- } \\
\text { try group on superselection sec- } \\
\text { tors, which is characterized by a } \\
\text { homomorphism } \omega_{1}: G \rightarrow \Gamma_{1}\end{array}$ & $\begin{array}{l}\text { Abelian phases } A_{x}, A_{y}, A_{z} \text { in the } \\
\text { honeycomb lattice model }\end{array}$ \\
\hline 2 & $\begin{array}{l}\text { The action of } G \text { on each super- } \\
\text { selection sector } a \text { is described by } \\
\text { a central extension } G_{a} ; \text { the whole } \\
\text { set of extensions is characterized } \\
\text { by an element } \omega_{2} \in H^{2}\left(B G, \Gamma_{2}\right)\end{array}$ & a number of models in $[95,96$ \\
\hline 3 & $\begin{array}{l}\text { Effective topological action for a } \\
G \text {-gauge field; such actions are } \\
\text { classified by } H^{4}(B G, \mathbb{Z})\end{array}$ & Integer quantum Hall effect \\
\hline 4
\end{tabular}

Table 6: Four levels of symmetry breaking in a two-dimensional quantum system.

group $G$ is assumed to be compact.) An analogue of the Chern-Simons action exists even for finite groups. In this case, $B G=K(G, 1)$ (where $K(G, n)$ is the Eilenberg-MacLane space) and $H^{4}(B G, \mathbb{Z}) \cong H^{3}(G, \mathrm{U}(1))$ (where the first $H$ refers to the topological cohomology and the second to the group cohomology).

The four-level classification of symmetry-breaking phenomena is summarized in Table 6. It has the flavor of topological obstruction theory (see e.g. 98). One may hypothesize that there is a somehow relevant topological space $Y$ such that $\pi_{1}(Y)=\Gamma_{1}, \pi_{2}(Y)=\Gamma_{2}, \pi_{3}(Y)=0$, $\pi_{4}(Y)=\mathbb{Z}$, and all higher homotopy groups vanish. We now argue that the analogy with obstruction theory has precise mathematical meaning in dimensions 1 and 2.

Suppose that the symmetry remains unbroken in dimension 0, but can be removed (if so is desired) by introducing a small perturbation to the Hamiltonian. That is obviously possible if the system consists of spins, while the $\mathbb{Z}_{2}$ symmetry associated with fermions can never be removed. The properties that survive the perturbation are described by a unitary modular category (UMC) $\mathcal{A}$ and a chiral central charge $c_{-}$, but we ignore the latter.

The action of the symmetry group $G$ on superselection sectors can be described algebraically as well as topologically. To begin with algebra, consider the 2 -groupoid $\Gamma=\operatorname{Aut}(\mathcal{A})$. It has a single object, the morphisms are invertible unitary braided tensor functors $\mathcal{A} \rightarrow \mathcal{A}$, and the 2-morphisms are natural isomorphisms between such functors. ${ }^{28}$ If the functors are considered up to isomorphisms, we obtain the group $\Gamma_{1}$ that was mentioned above. The Abelian group $\Gamma_{2}$ consists of all automorphisms of the identity functor. Note that $\Gamma_{1}$ may act on $\Gamma_{2}$ in a nontrivial

\footnotetext{
${ }^{28}$ As explained to me by Ezra Getzler, higher groupoids may be nicely defined as Kan complexes [99]; this approach also allows one to endow the morphism sets with topology. In the definition of $\Gamma$ no topology is assumed. It would be interesting to see what changes (if anything) when a natural topology is included.
} 
way. The 2-groupoid $\Gamma$ is characterized by this action and a cohomology class $h \in H^{3}\left(\Gamma_{1}, \Gamma_{2}\right)$.

On the topological side, one can consider the classifying space $X=B \Gamma$, which is a topological space with a basepoint. I claim that $X$ is connected, $\pi_{1}(X)=\Gamma_{1}, \pi_{2}(X)=\Gamma_{2}$, and all higher homotopy groups vanish. Furthermore, the action of $G$ on superselection sectors is described by a continuous map $f: B G \rightarrow X$ defined up to a homotopy (some explanation is given below). One may ask if $f$ is homotopic to a constant map. The first obstruction to such a homotopy is given by a homotopy class of maps $\omega_{1}: B G \rightarrow K\left(\Gamma_{1}, 1\right)$; such classes are in one-to-one correspondence with group homomorphisms $G \rightarrow \Gamma_{1}$. If this obstruction vanishes, one can define $\omega_{2} \in H^{2}\left(B G, \Gamma_{2}\right)=H^{2}\left(G, \Gamma_{2}\right)$. If $\omega_{2}=0$, then $f$ is homotopic to identity, or equivalently, the action of $G$ on $\mathcal{A}$ is trivial.

Note that $X$ can be represented as a fibration with base $K\left(\Gamma_{1}, 1\right)$ and fiber $K\left(\Gamma_{2}, 2\right)$. Its structure is characterized by the element $h \in H^{3}\left(\Gamma_{1}, \Gamma_{2}\right)$. If $h \neq 0$, then the fibration does not have a cross section. In this case, the homomorphism $\omega_{1}: G \rightarrow \Gamma_{1}$ must satisfy a certain constraint, namely the inverse image of $h$ by $\omega_{1}$ must vanish. Thus, some seemingly possible types of symmetry breaking in dimension 1 may be forbidden due to an obstruction in dimension 2.

In conclusion, I conjecture a "physical" interpretation of the space $X$ and the map $f$. Specifically, $X$ parametrizes quantum states in the universality class described by the unitary modular category $\mathcal{A}$. Each point of $X$ is associated with a set of states that differ from each other locally; thus all finite-energy excitations of a given gapped Hamiltonian are represented by the same point. The original unperturbed system corresponds to some $x_{0} \in X$. To define the map $B G \rightarrow X$, let us assume that $G$ is a compact Lie group which acts on each spin independently according to a faithful representation $G \rightarrow \mathrm{U}(m)$. The Hilbert space of the spin, $\mathbb{C}^{m}$ may be embedded in a large space $\mathbb{C}^{M}$. Each embedding $u$ has an associated state $g(u) \in X$. The space $E$ of embeddings is contractible in the limit $M \rightarrow \infty$, therefore its quotient with respect to the natural $G$-action may be regarded as a model of $B G$. Furthermore, since $x_{0}$ is symmetric, the map $g: E \rightarrow X$ factors through $B G$. Thus the map $f: B G \rightarrow X$ is defined.

Remark F.1. It is not clear how to define the "extended classifying space" $Y$ that has nontrivial homotopy in dimension 4 . One may conjecture that $Y=X \times K(\mathbb{Z}, 4)$, but this definition seems contrived. It may well be the case that the very idea to classify the Chern-Simons action as symmetry breaking in dimension 4 is wrong. Indeed, the Chern-Simons action is not fully described by homotopy theory because the fundamental homology class of the source (physical) manifold is involved.

\section{Acknowledgments}

During the years this work was in progress I received genuine interest and words of encouragement from John Preskill, Michael Freedman, Mikhail Feigelman, Grigory Volovik, and many other people. I thank Andreas Ludwig for having convinced me in the importance of the chiral central charge in the study of topological order. A conversation with Dmitri Ivanov was essential for understanding the difference between non-Abelian anyons and Majorana vortices in twodimensional $p$-wave superconductors. I also thank Matthew Fisher, Nicholas Read, and Xiao-

Gang Wen for helpful discussions. I am especially grateful to Jean Bellissard who read a preliminary version of the manuscript and made some valuable comments; in particular, he directed 
me to Ref. [50]. This work was supported in part by the National Science Foundation under Grant No. EIA-0086038 and by the Army Research Office under grant No. W911NF-04-1-0236.

\section{References}

[1] J. Bellissard, A. van Elst, H. Schulz-Baldes, "The noncommutative geometry of the quantum Hall effect," J. Math. Phys. 35, 5373-5451, (1994), cond-mat/9411052.

[2] F. Wilczek, "Magnetic flux, angular momentum, and statistics", Phys. Rev. Lett. 48, 11441146 (1982).

[3] F. Wilczek, "Remarks on dyons", Phys. Rev. Lett. 48, 1146-1149 (1982).

[4] G. Moore, N. Seiberg, "Classical and quantum conformal field theory", Commun. Math. Phys. 123 no. 2, 177-254 (1989).

[5] E. Witten, "Quantum field theory and the Jones polynomial", Commun. Math. Phys. 121 no. 3, 351-399 (1989).

[6] K. Fredenhagen, K. H. Rehren, B. Schroer, "Superselection sectors with braid group statistics and exchange algebras, I: General theory", Commun. Math. Phys. 125 no. 2, 201-226 (1989).

[7] J. Fröhlich, F. Gabbiani, "Braid statistics in local quantum field theory", Rev. Math. Phys. 2 no. 3, 251-353 (1990).

[8] R. B. Laughlin, "Anomalous quantum Hall effect - an incompressible quantum fluid with fractionally charged excitations," Phys. Rev. Lett. 50, 1395-1398 (1983).

[9] L. Saminadayar, D. C. Glattli, Y. Jin, B. Etienne, "Observation of the e/3 fractionally charged Laughlin quasiparticles", Phys. Rev. Lett. 79, 2526-2529 (1997), cond-mat/9706307.

[10] R. de-Picciotto, M. Reznikov, M. Heiblum, V. Umansky, G. Bunin, D. Mahalu, "Direct observation of a fractional charge", Nature 389, 162-164 (1997), cond-mat/9707289.

[11] B. I. Halperin , "Statistics of quasiparticles and the hierarchy of fractional quantized Hall states", Phys. Rev. Lett. 52, 1583-1586 (1984).

[12] D. Arovas, J.R. Schrieffer, and F. Wilczek, "Fractional statistics and the quantum Hall effect", Phys. Rev. Lett. 53, 722-723 (1984).

[13] F. E. Camino, W.Zhou, V. J. Goldman, "Direct observation of fractional statistics in two dimensions", cond-mat/0502406.

[14] G. Moore, N. Read, "Nonabelions in the fractional quantum Hall effect," Nucl. Phys. B 360, 362-396 (1991). 
[15] G. Moore, N. Read, "Fractional quantum Hall effect and nonabelian statistics", hep-th/9202001.

[16] M. B. Hastings, "Lieb-Schultz-Mattis in higher dimensions", Phys. Rev. B 69, 104431 (2004), cond-mat/0305505.

[17] P. W. Anderson, "Resonating valence bond: A new kind of insulator?", Mat. Res. Bull. 8, 153-160 (1973).

[18] P. W. Anderson, "The resonating valence bond in $\mathrm{La}_{2} \mathrm{CuO}_{4}$ and superconductivity", Science 235, 1196-1198 (1987).

[19] R. Moessner, S. L. Sondhi, "Resonating valence bond phase in the triangular lattice quantum dimer model", Phys. Rev. Lett. 86, 1881-1884 (2001), cond-mat/0007378.

[20] N. Read, B. Chakraborty, "Statistics of the excitations of the resonating-valence-bond state", Phys. Rev. B 40, 7133-7140 (1989).

[21] T. Senthil, M.P. A. Fisher, "Fractionalization in the cuprates: Detecting the topological order", Phys. Rev. Lett. 86, 292-295 (2001), cond-mat/0006481.

[22] D. A. Bonn, J.C. Wynn, B.W. Gardner, Yu-Ju Lin, Ruixing Liang, W. N. Hardy, J.R. Kirtley, K. A. Moler, "A limit on spin-charge separation in high- $T_{c}$ superconductors from the absence of a vortex-memory effect", Nature 414, 887-889 (2001).

[23] R. Coldea, D. A. Tennant, A. M. Tsvelik, Z. Tylczynski, "Experimental realization of a 2d fractional quantum spin liquid", Phys. Rev. Lett. 86, 1335-1338 (2001), cond-mat/0007172.

[24] A. Yu. Kitaev, "Fault-tolerant quantum computation by anyons", Annals of Physics 303 no. 1, 2-30 (2003), quant-ph/9707021.

[25] S. Doplicher, J.E. Roberts, "Why there is a field algebra with a compact gauge group describing the superselection structure in particle physics", Commun. Math. Phys. 131 no. 1, 51-107 (1990).

[26] S. Doplicher, J.E. Roberts, "A new duality theory for compact groups" Invent. Math. 98 no. 1, 157-218 (1989).

[27] M. H. Freedman, M. Larsen, Z. Wang, "A modular functor which is universal for quantum computation", Commun. Math. Phys. 227 no. 3, 605-622 (2002), quant-ph/0001108.

[28] T. Einarsson, "Fractional statistics on a torus", Phys. Rev. Lett. 64, 1995-1998 (1990).

[29] A. Ocneanu, unpublished.

[30] P. Etingof, D. Nikshych, V. Ostrik, "On fusion categories", math.QA/0203060.

[31] N. Read, E. Rezayi, "Beyond paired quantum Hall states: Parafermions and incompressible states in the first excited Landau level", Phys. Rev. B, 59 8084-8092 (1999), cond-mat/9809384. 
[32] R.W. Ogburn, J. Preskill, "Topological quantum computation", Lecture Notes in Computer Science 1509, C. P. Williams (ed.) QCQC'98, pp. 341-356, Springer-Verlag (1999).

[33] J. Preskill, "Fault-tolerant quantum computation", quant-ph/9712048.

[34] C. Mochon, "Anyon computers with smaller groups", Phys. Rev. A 69, 032306 (2004), quant-ph/0306063.

[35] M. H. Freedman "A magnetic model with a possible Chern-Simons phase", Commun. Math. Phys. 234 no. 1, 129-183 (2003), quant-ph/0110060.

[36] M. Freedman, C. Nayak, K. Shtengel, K. Walker, Z. Wang, "A Class of P, T-Invariant Topological Phases of Interacting Electrons", cond-mat/0307511.

[37] M. Freedman, C. Nayak, K. Shtengel, "Non-Abelian topological phases in an extended Hubbard model", cond-mat/0309120.

[38] B. Douçot, J. Vidal, "Pairing of Cooper Pairs in a Fully Frustrated Josephson Junction Chain", Phys. Rev. Lett. 88, 227005 (2002)cond-mat/0202115.

[39] L. B. Ioffe, M. V. Feigel'man, "Possible realization of an ideal quantum computer in Josephson junction array", Phys. Rev. B 66, 224503 (2002), cond-mat/0205186.

[40] B. Douçot, L. B. Ioffe, M. V. Feigel'man, "Topological order in the insulating Josephson junction array", Phys. Rev. Lett. 90, 107003 (2003), cond-mat/0211146.

[41] B. Douçot, L. B. Ioffe, M. V. Feigel'man, "Discrete non-Abelian gauge theories in twodimensional lattices and their realizations in Josephson-junction arrays", Phys. Rev. B 69, 214501 (2004), cond-mat/0302104.

[42] L.-M.Duan, E. Demler, M. D. Lukin Phys. Rev. Lett. 91, 090402 (2003), cond-mat/0210564.

[43] D. J. Thouless, M. Kohmoto, M. P. Nightingale, M. den Nijs, "Quantized Hall conductance in a two-dimensional periodic potential," Phys. Rev. Lett. 49, 405-408 (1982).

[44] J.E. Avron, R.Seiler, B.Simon, "Homotopy and quantization in condensed matter physics," Phys. Rev. Lett. 51, 51-53, (1983).

[45] A. Connes, "Noncommutative Geometry", Academic Press (1994).

[46] G. E. Volovik, "Analog of quantum Hall-effect in a superfluid ${ }^{3}$ He film" Zh. Eksp. Teor. Fiz. 94 no. 9, 123-137 (1988) (in Russian) [English translation: Sov. Phys. JETP 67, 1804 (1988)].

[47] G. E. Volovik, V. M. Yakovenko, "Fractional charge, spin and statistics of solitons in superfluid ${ }^{3}$ He film", J. Phys.: Condens. Matter 1, 5263-5274 (1989).

[48] N. Read, D. Green, "Paired states of fermions in two-dimensions with breaking of parity and time reversal symmetries, and the fractional quantum Hall effect," Phys. Rev. B 61, 10267 (2000), cond-mat/9906453. 
[49] A. M. Tsvelik, "Quantum field theory in condensed matter physics", Cambridge University Press, 2nd edition (2003).

[50] E. H. Lieb, "Flux phase of the half-filled band", Phys. Rev. Lett. 73, 2158-2161 (1994), cond-mat/9410025.

[51] B. I. Halperin "Quantized Hall conductance, current-carrying edge states, and the existence of extended states in a two-dimensional disordered potential," Phys. Rev. B 25, 2185-2190 (1983).

[52] Y. Hatsugai, "Chern number and edge states in the integer quantum Hall effect," Phys. Rev. Lett. 71, 3697-3700 (1993).

[53] H. Schulz-Baldes, J. Kellendonk, T. Richter, "Simultaneous quantization of edge and bulk Hall conductivity," J. Phys. A: Math. Gen. 33 L27-L32 (2000), cond-mat/9912186.

[54] J. Kellendonk, T. Richter, H.Schulz-Baldes, "Edge current channels and Chern numbers in the integer quantum Hall effect," Rev. Math. Phys. 14 no. 1, 87-119 (2002).

[55] G. E. Volovik, "Quantum Hall and chiral edge states in thin ${ }^{3}$ He-A film", Pis'ma v ZhETF 55 no. 6, 363-367 (1992) [JETP Letters, 55 368-373 (1992)].

[56] C. L. Kane, M. P. A. Fisher, "Quantized thermal transport in the fractional quantum Hall effect", Phys. Rev. B 55, 15832-15837 (1997), cond-mat/9603118.

[57] A. Cappelli, M.Huerta, G.R.Zemba, "Thermal transport in chiral conformal theories and hierarchical quantum Hall states", Nucl. Phys. B 636 no. 3, 568-582 (2002), cond-mat/0111437.

[58] U. Sivan, Y. Imry, "Multichannel Landauer formula for thermoelectric transport with application to thermopower near the mobility edge", Phys. Rev. B 33, 551-558 (1986).

[59] L. Alvarez-Gaume, E. Witten, "Gravitational anomalies", Nucl. Phys. B 234 no. 2, 269330 (1984).

[60] G. E. Volovik, "The gravitational topological Chern-Simons term in a film of superfluid ${ }^{3}$ He-A", Pis'ma v ZhETF 51 no. 2, 111-114 (1990) (in Russian) [English translation: JETP Letters, 51 125-128 (1990)].

[61] K. H. Rehren, "Braid group statistics and their superselection rules" in The algebraic theory of superselection sectors, D. Kastler (ed.) Proceedings Palermo 1989, pp. 333-355, World Scientific Publishing (1990).

[62] E. Verlinde, "Fusion rules and modular transformations in 2D conformal field theory", Nucl. Phys. B 300, 360-376 (1988).

[63] P. Di Francesco, P. Mathieu, D. Sénéchal, "Conformal field theory", Springer (1996).

[64] A. Yu. Kitaev, "Unpaired Majorana fermions in quantum wires", cond-mat/0010440. 
[65] D. A. Ivanov, "Non-Abelian statistics of half-quantum vortices in $p$-wave superconductors", Phys. Rev. Lett. 86, 268-271 (2001), cond-mat/0005069.

[66] N. Reshetikhin, V. G. Turaev, "Invariants of 3-manifolds via link polynomials and quantum groups", Inventiones Mathematicae 103 no. 3, 547-597 (1991).

[67] K. Walker, "On Witten's 3-manifold invariants", http://canyon23.net/math/ (1991).

[68] V. G. Turaev, "Quantum invariants of knots and 3-manifolds" (de Gruyter Studies in Mathematics 18), de Gruyter (1994).

[69] V. Kalmeyer, R. B. Laughlin, "Equivalence of the resonating-valence-bond and fractional quantum Hall states", Phys. Rev. Lett. 59, 2095-2098 (1987).

[70] M. V. Feigel'man, A. Yu. Kitaev, unpublished.

[71] R. B. Laughlin, "Quantized Hall conductivity in two dimensions," Phys. Rev. B 23, 56325633 (1981).

[72] J. E. Avron, R. Seiler, B. Simon, "Quantum Hall effect and the relative index of projections," Phys. Rev. Lett. 65, 2185-2189 (1990).

[73] J.E. Avron, R. Seiler, B. Simon, "Charge deficiency, charge transport and comparison of dimensions," Commun. Math. Phys. 159 no. 2, 399-422 (1994), physics/9803014.

[74] J.E. Avron, R. Seiler, B. Simon, "The index of a pair of projections," J. Func. Analysis 120, 220-237 (1994).

[75] E. M. Rains, "Correlation functions for symmetrized increasing subsequences", math.CO/0006097.

[76] D. A. Rokhsar, S. A. Kivelson, "Superconductivity and the quantum hard-core dimer gas", Phys. Rev. Lett. 61, 2376-2379 (1988).

[77] E. H. Lieb, D. W. Robinson, "The finite group velocity of quantum spin systems", Commun. Math. Phys. 28, 251-257 (1972).

[78] R. Haag, "Local quantum physics", Springer Verlag, 2nd edition (1996).

[79] B. Bakalov, A. Kirillov Jr., "Lectures on tensor categories and modular functors" (University Lecture Series 21), American Mathematical Society (2001).

[80] C. Kassel, "Quantum groups" (Graduate Texts in Mathematics 155), Springer Verlag (1995).

[81] J. Preskill, "Topological quantum computation", (Chapter 9 of Lecture notes on quantum computation), http://www.theory.caltech.edu/people/preskill/ph229/\#lecture (2004).

[82] S. Mac Lane, "Categories for the working mathematician" (Graduate Texts in Mathematics 5), Springer Verlag, 2nd edition (1998). 
[83] J. D. Stasheff, "Homotopy associativity of $H$-spaces" (parts I and II), Trans. Amer. Math. Soc. 108, 275-292 and 293-312 (1963).

[84] J.-L. Loday, "Realization of the Stasheff polytope" Arch. Math. (Basel) 83 no. 3, 267-278 (2004), math.AT/0212126.

[85] V. G. Drinfeld, "Quantum groups", Proceedings of the International Congress of Mathematicians, Berkeley 1986, pages 798-820, Amer. Math. Soc. (1987).

[86] F. A. Bias, P. van Driel, M. de Wild Propitius, "Quantum symmetries with discrete gauge symmetries", Phys. Lett. B 280, 63-70 (1992), hep-th/9203046.

[87] R. Dijkgraaf, V. Pasquier, P. Roche, "Quasi Hopf algebras, group cohomology and orbifold models", Nucl. Phys. B (Proc. Suppl.) 18, 60-72 (1991).

[88] F. A. Bias, P. van Driel, M. de Wild Propitius, "Anyons in discrete gauge theories with Chern-Simons term", Nucl. Phys. B 393, 547-570 (1993), hep-th/9203047.

[89] K. Fredenhagen, K. H. Rehren, B. Schroer, "Superselection sectors with braid group statistics and exchange algebras, II: Geometric aspects and conformal covariance", Rev. Math. Phys. special issue 1, 113-157 (1992).

[90] C. Vafa, "Toward classification of conformal theories", Phys. Lett. B 206, 421-426 (1988).

[91] L. Crane, D. N. Yetter, "Deformations of (bi)tensor categories", Cahiers Topologie Géom. Différentielle Catég. 39 no. 3, 163-180 (1998), q-alg/9612011.

[92] A. A. Davydov, "Twisting of monoidal structures", q-alg/9703001.

[93] D. N. Yetter, "Functorial knot theory", (Series on Knots and Everything, 26), World Scientific Publishing (2001).

[94] A. Rosenberg, "The existence of fiber functors", Gelfand Mathematical Seminars 19961999, 145-154, Birkhäuser Boston (2000).

[95] X.-G. Wen, "Quantum orders and symmetric spin liquids", Phys. Rev. B 65, 165113 (2002), cond-mat/0107071.

[96] X.-G. Wen, "Quantum orders in an exact soluble model", Phys. Rev. Lett. 90, 016803 (2003), quant-ph/0205004.

[97] R. Dijkgraaf, E. Witten, "Topological gauge theories and group cohomology", Commun. Math. Phys. 129 no. 2, 393-429 (1990).

[98] A. Hatcher, "Algebraic topology", Cambridge University Press (2002).

[99] J. P. May, "Simplicial objects in algebraic topology" (Chicago Lectures in Mathematics Series) University of Chicago Press, Reprint edition (1993). 